



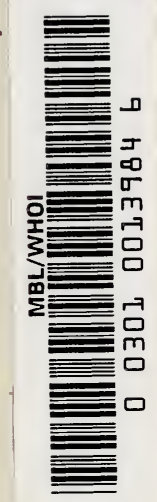





LES

\section{DIATOMÉES FOSSILES}

D'AUVERGNE 



\section{LES}

\section{DTATOMEES FOSSILES}

\section{D'AUVERGNE}

PAR

\section{Le Frère HÉRIBAUD JOSEPH}

\section{PROFESSEUR AU PEXsionxat dE CLERMONT-FERRAND} LAUREAT DE L'INSTITUT DE FRANCE

(Académie des Sciences)

MEMBRE HOXORAIRE ÉLU DE L'ACADÉMIE DE CLERMONT-FERRAND

ET DE LA Société botanique de Fraxce

ANCIEx DHRETEUR DE L'ACADÉME INTERNATIONALE

DE GÉOGRAPHIE BOTANIQLE

\section{DEO scientiarum Domino laus et glorix.}

\section{AVEC 2 PLANCHES}

DESSINÉES PAR LE COMMANDANT MAURICE PERAGALLO

\section{PrIX : 5 Francs}

\begin{tabular}{|c|c|}
\hline & \\
\hline & \\
\hline PENSIONNAT & LIBRAIRIE DES SCIENCES NATURELLES \\
\hline DES FRĖES DES ÉCOLES CHRÉTIENNES & PAUL KLINCKSIECK \\
\hline Rue Godefroy-de-Bouillon & 3, Rue Corneille, 3 \\
\hline & \\
\hline & \\
\hline
\end{tabular}





\section{PRÉFACE}

La découverte rẻcente du vaste dépòt de Celles, près la gare de Neussargues, par notre distingué compatriote, M. Jean Pagès-Allary, propriétaire-industriel à Murat, et celle du beau dépòt de la Bade, près de Collandre, par M. A. Chareton-Chaumeil, avoué-géologue à Langres, nous a engagé à poursuivre l'étude captivante des Diatomées de notre belle province, et compléter ainsi, dans la mesure du possible, le mémoire publiẻ en 1893 sur ce groupe d'Algues inférieures, mémoire accueilli avec trop de faveur par les diatomistes français et étrangers, et par l'Institut de France (Académie des Sciences).

L'étude des dépòts de Celles et de la Bade a été faite avec le plus grand soin, d'après de nombreux échantillons pris sur les divers points de leur masse, et à l'aide des meilleures lentilles.

Malgré le nombre considérable de préparations examinees (environ 200), nous n'avons pas la prétention d'avoir relevẻ la série complète des espèces qu'ils contiennent; ces deux dépòts, comme ceux d'Auxillac, de Neussargues et du Puy de Mur, ménagent encore bien des surprises 
agréables aux diatomistes qui les étudieront après nous; ce sont des mines inépuisables.

A la suite des deux dépóts cantaliens, deux autres, appartenant au Puy-de-Dóme, ont été l'objet de nos recherches : ce sont les dépóts de Perrier, près d'Issoire, et celui du ravin des Egravats, près de la Grande Cascade du Mont-Dore; toutefois, leur florule n'offre point la variété des dépóts de Celles et de la Bade; nous le constaterons plus loin.

Dans l'espoir de trouver quelques noureautés dans les riches dépôts d'Auxillac, de Neussargues, de Verneuges et du Puy de Nur, nous en avons examiné des échantillons nombreux pris á différentes profondeurs, ou provenant d'affleurements découverts depuis la publication des Diatomées d'A ucergne. Le résultat a dépassé nos espérances; en effet, avec les deux genres Opephora et Campylosira, nouveaux pour notre flore diatomique, ces dépóts nous ont encore procuré un bon nombre de formes très intéressantes, parmi lesquelles plusieurs sont inćdites.

Les deux remarquables planches de ce némoire sont dues au talent bien connu de M. le Commandant Maurice Peragallo. Nous sommes lieureux de lui exprimer ici nos meilleurs remerciements pour le service précieux qu'il nous a rendu avec la plus parfaite amabilité.

Nous offrons aussi, à M. Pagès-Allary et á M. CharetonChaumeil, l'expression de notre bien vive reconnaissance pour nous avoir communiqué les prémices de leur impor- 
tante découverte, nous permettant ainsi d'établir notre droit de priorité au sujet de l'étude des dépóts de Celles et de la Bade.

Merci à notre aimable et savant compatriote, M. Pierre Marty, du chàteau de Caillac, pour les éléments d’étude qu'il a eu l'amabilité de nous procurer; parmi les échantillons recus, et provenant du dépòt de Neussargues, nous avons eu le plaisir de découvrir le genre Opephor'a, encore peu connu des diatomistes français.

Enfin, nous devons ả M. Charles Saintigny, agent-voyer en retraite, plusieurs échantillons du dépót de Verneuges (Puy-de-Dỏme), dépót dont il est le propriétaire et qu'il se propose d'exploiter au point de vue industriel; l'un des échiıntillons, pris à 4 mètres de profondeur, nous a fourni 3 ou 4 formes inédites fort curieuses, avec plusieurs espèces que nous n'avions pas constatées dans l'èchantillon étudié en 1890 , et transmis par M. l'abbé R. Crègut.

L'examen d'un assez grand nombre de récoltes de Diatomées vivantes ne nous ayant donné aucune forme nouvelle à ajouter aux espèces mentionnées dans notre travail de 1393, nous avons dủ nous borner á publier, dans les pages suivantes, le résultat de nos études sur les Diatomées fossiles.

Pensionnat des Frères de Clermont-Ferrand.

25 Février 1902.

F. HÉribaud Josepil. 

LES

\section{DILTOMÉES FOSSILES}

\section{I)'AUVERGNE}

\section{DÉPOTS ÉTUDIÉS.}

Les dépòts diatomifères qui font l'objet de ce mémoire sont d'abord: les dépòts de Celles, de la Bade, de Perrier et du ravin des Egravats, découverts postérieurement à la publication des Diatomées d'Auvergne (1893); puis, les dépóts du Puy de Mur, de Verneuges, de la Bourboule, d'Auxillac et de Neussargues, déjả connus, mais dont la florule exigeait de nouvelles investigations.

I.

Dépôts nouveaux pour la Flore d'Auvergne

\section{DÉPOT DE CELLES (Cantal)}

(Pliocène supérieur).

Le dépòt de Celles, découvert au mois de juin 1901 par M. Pagès-Allary, est situé près de la gare de Neussargues, á l'altitude de 870 mètres, à l'entrée du bois de Celles et au S.-O. par rapport à la gare.

D'après les documents précis fournis par notre honorable compatriote, ce dépòt doit avoir une grande étendue, 
comme le prouvent les sondages effectués sur plusieurs points, et assez distants les uns des autres pour en conclure que sa surface est au moins égale à celle du dépòt d'Auxillac.

La plus grande épaisseur constatée jusqu'à présent est de 10 mètres, mais il est fort probable qu'elle sera trouvée, sur d'autres points, supérieure á ce chiffre.

Une couche de terre végétale, et des accumulations morainiques formées de roches d'origine très diverse et plus ou moins fragmentées, le recouvrent. A un certain niveau, la masse diatomifère est traversée, dans le sens horizontal, par un lit de cendres volcaniques (cinérites), de 20 a 25 centimètres d'épaisseur.

Sur un échantillon reçu nous avons constaté une bonne empreinte de feuille d'un Fagus, probablement Fagus pliocenica; il est donc évident que le dépòt renferme des végétaux fossiles, plus ou moins abondants. Or, la constatation de feuilles fossiles et l'existence d'une couclie de cinérite intercalée dans l'épaisseur de la masse sont une preuve certaine que le dépòt a été remanié. Repris par les eaux à l'époque pliocène, il a été entrainé dans la dépression formée par une puissante coulée basaltique, épanchée perpendiculairement á la direction du courant boueux diatomifère, qui l'a retenu à la manière d'une digue gigantesque. La fossilisation des feuilles n'a pu s'effectuer qu'au moment où la masse à Diatonées s'est déposée dans la dépression qu'elle occupe actuellement; d'où il résulte que la florule phanérogamique est postérieure à la florule diatomique, c'est-á-dire à la formation du dépót. Au cours de ce mémoire nous aurons ì revenir sur ce point très instructif, concernant les deux florules des dépóts diatomifères remaniés.

Le dépòt repose directement sur une assise de terre noire et compacte dont la formation ne s'explique pas facilement, à moins d'y voir simplement le sol tourbeux d'un ancien marais, modifié a la fois par les émissions volcani- 
ques et par une forte pression; mais, ce n'est lá qu'une indication pour nos compatriotes géologues; n'ayant pas étudié ce point spécial de la question, nous leur laissons le soin de l'élucider.

A l'état sec, le dépót est d'un blanc grisatre, pulvérulent, onctueux au toucher et d'une pureté remarquable, à en juger par les échantillons étudiés. A notre avis, il est de tout premier ordre au point de vue des applications industrielles, bien supérieur aux meilleurs dépòts allemands, trop longtemps utilisés en France, au préjudice de ceux d'Auvergne.

Les deux Diatomées caractéristiques, et qui forment la plus grande partie de la masse du dépòt, sont: le Cyclotella Iris avec sa variété integra, et le Melosira spiralis, avec ses deux variétés hemispharica et spharica, extrêmement curieuses.

Ces Diatomées, à valves très épaisses, très résistantes, sont rarement fragmentées, et c'est précisément à cette particularité que le dépòt de Celles doit ses qualitės tout à fait supérieures. Les dépóts allemands, que nous avons beaucoup étudiés à l'occasion de la publication des Diatomées d'Aluvergne, sont formés en grande partie d'espèces appartenant aux genres Fragilaria, Navicula, Nitsschia, Synedra, etc., à valves minces, très fragiles, d'oil il résulte que les frustules sont presque toujours brisés; or, il est évident que, dans ces conditions, les dépòts allemands sont très inférieurs à celui de Celles, au point de vue spécial du pouvoir absorbant. Dans la plupart des applications industrielles, en particulier pour la fabrication de la dynamite, le dépòt cantalien doit leur ètre préféré.

Voici la série des espèces et variẻtés observées dans les échantillons examinés (1) :

(1) Les Diatomées déjả connues sont imprimées en itulique, et les espèces et variétés nouvelles en égyptien. 
Cocconeis lineata Grun. var. euglypta Grun. (Diat. d'Auv. p. 46). AR.

Gomphonema biventralis F. Hérib. et M. Per. (Pl. VIII, fig. 3). - Analogue au Gomph. acuminatumi Ehrb., mais présentant deux ètranglements au lieu d'un seul. Longueur $75 \mu$, largeur de la tête $18 \mu$; les deux parties ventrales ont respectivement 15 et $10 \mu$. Raphé presque invisible. Aréa assez large, s'élargissant ou se rétrécissant dans le sens des bords de la valve. Stries nettement granulées, au nombre de 9 en $10 \mu$ au centre, un peu plus écartées á la tẻte, et plus serrées au contraire à l'autre extrémité. $R$.

Gomphonema insigne Greg. var. acuminata M. Per. et F. Hérib. (Pl. VIII, fig. 4). - Diffère du type par sa forme nettement anguleuse, et dont la tête est brusquement diminuée pour se terminer en pointe. Longueur $65 \mu$. Stries fines, au nombre de 16 en $10 \mu$. RR.

Gomph. acuminatum Ehrb. (Diat. d'Auv., p. 53). R.

- geminatum Ag. nec Ktz. (Diat. d'Auv., p. 52). R.

- intricatum Ktz. (Diat. d'Auv., p. 57). RR.

- subtile Ehrb. (Diat. d'Auv., p. 58). RR.

- subclavatum Grun. (Diat. d'Auv., p. 55). R.

- - var. major F. Hérib. et M. Per. - Se distingue du type par sa plus grande longueur, et par les stries moins serrées. Longueur $66 \mu$. Striess au nombre de 11 en $10 \mu$. R.

Amphor'a affinis Ktz. (Diat. d'Auv., p. 63). AR.

Gymbella scotica IV. Sm.; V. H. pl. 2, fig. 21. R. - aspera Ehrb. (Diat. d'Auv., p. 69). R.

- cymbiformis Ehrb. (Diat. d'Auv., 1'. 69). R.

Encyonema Girodi F. Hérib. (Pl. VII, fig 18). Espèce très distincte. Longueur $80 \mu$, largeur $15 \mu$; ventre et dos gibbeux ; gibbosité du ventre plus sensible que celle 
du dos. Raphé droit. Nodules terminaux en forme de virgule, placés contre le bord dorsal et assez loin de l'extrénité de la valve. Aréa s'élargissant autour du nodule central et des nodules terminaux. Stries de la face dorsale convergentes, et au nombre de $61 / 2$ a 7 en $10 \mu$ au centre, mais plus serrées aux extrémités, nettement granulées et plus fortes vers les bords que vers le raphé. Stries de la face ventrale au nombre de $S$ en $10 \mu$ au centre, où elles sont convergentes, puis divergentes pour converger autour des nodules terminaux, où elles sont beaucoup plus serrées qu'au centre, moins nettement granulées que celles de la face dorsale.

Nous dédions cette belle Diatomée à M. le $\mathrm{D}^{\mathrm{r}}$ Paul Girod, professeur de Botanique á l'Université de Clermont, en reconnaissance de l'intérêt qu'il nous a constamment témoigné au cours de nos recherches sur les Diatomées d'Auvergne.

Navicula cellesensis F. Hérib. et M. Per. (Pl. VII, fig. 13). - De forme lenticulaire, à extrémités très largement arrondies. Longueur $58 \mu$, largeur $15 \mu$. Raphé filiforme, a nodules terminaux petits, en forme de crochets, et dont les deux parties se recourbent dans le même sens pour former le nodule central. Stries marginales fines, au nombre de 12 en $10 \mu$, laissant une large aréa centrale limitée par des lignes á peu près parallèles aux bords de la valve. $R R$.

La Navicule du dépót de Celles, ressemble à une petite forme du Navicula instabilis A. Sch., Atl., pl. 43, fig. 39, qui est une espèce marine de l'Amérique du Sud.

Navicula Gomontiana F. Hérib. (Pl. VII, fig. 14). - De forme lenticulaire, à extrémités assez largement arrondies et légèrement subrostrées. Longueur $57 \mu$, largeur $23 \mu$. Raphé porté sur une surélévation de la valve, assez fort au nodule central et s'atténuant jusqu'aux nodules terminaux qui sont arrondis et très petits. Stries 
convergentes, courbes, paraissant lisses, au nombre de 6 en $10 \mu$ au centre, mais plus serrées aux extrémités, ou elles arrivent jusqu'au surélèvement de la valve portant le raphé, tandis qu'au centre elles laissent une aréa en forme de losange. R.

Notre Navicula Gomontiana est analogue au Navicula Placentula d'Ehrenberg, mais il s'en distingue nettement par la forme du raphé et par les extrémités de la valve.

Cette Navicule est dédiée à M. Maurice Gomont, l'auteur bien connu de la savante Monographie des Oscillariées et de plusieurs autres publications algologiques, notamment d'un mémoire très remarquable sur les Algues du Cantal.

Navicula nobilis Ehrb. (Diat. d'Auv., p. 80). R.

- major Kitz. (Diat. d'Auv., p. 82). AC.

- viridis Kitz. (Diat. d'Auv., p. 83). R.

-- rupestris Ktz. (Diat. d'Auv., p. 84). RR.

- acrospheria Bréb. var. lavois (Diat. d'Auv., p. 93). R.

- rhomboides Ehrb. (Diat. d'Auv., p. 110). RR.

- transversa A. Sich. RR.

-- limosa Ktz. var. gibberula Grun. (Diat. d'Auv., p. 112). RR.

- elliptica Ktz. (Diat. d'Auv., p. 104). AR.

Navicula Pagesi F. Hérib. (Pl. TII, fig. 7). - De forme largement elliptique. Longueur 50 a $75 \mu$, largeur 30 à $40 \mu$. Raphé très fin, non entouré de bourrelets siliccux, nodules terminaux petits, un peu éloignés des extrémités de la valve; nodule central formé de deux granules bien ronds. Aréa étroite, dilatée autour du nodule central. Sillons latéraux bien définis, allant en s'élargissant progressivement des extrémités au centre de la valve. Stries convergentes, au nombre de 9 en $10 \mu$, formées de granules jointifs dans tous les sens, mais alignés suivant la direction des stries; on peut dire également que les stries sont jointives, divisées en travers, chaque division 
formée par un granule. - Se distingue du Nacicula elliptica Kitz. par sa taille et par le nombre de ses stries. AC.

Pantocseck figure dans son ourrage sur les Diatomées fossiles de Hongrie, III, pl. 17, fig. 246, sous le nom de Navicula carpathorum, une forme qui ressemble assez bien au Navicula Pagesi, mais le raphé est très différent chez les deux espèces, et ne permet pas de les confondre.

Cette jolie Diatomée est dédiée à M. Jean Pagès-Allary, en souvenir de son intéressante découverte.

Pleurosigma attenuatum Kitz. (Diat. d'Auv., p.122).R. Epithemia turgida Kitz. (Diat. d'Auv., p. 124). AC.

- Hyndmannii WV. Sm. (Diat. d'Auv., p. 125). C.

- gibba Ehrb. (Diat. d'Auv., p. 126). R.

- Sorex Ktz. (Diat. d'Auv., p. 126). AR.

- Zebra Ktz. (Diat. d'Auv., p. 127). C.

- Argus Ktz. var. amphicephala Grun. (Diat. d'Auv., p. 127). R.

Eunotia pectinalis Rab. var. stricta Rab. (Diat. d'Aur., p. 132). AR.

- impressa Ehrb. var. angusta Grun. (Diat. d'Auv., p. 134). R.

- polyglyphis Ehrb. (Diat. d'Auv., p. 134). R.

Synedra Ulna Ehrb. (Diat. d'Aur., p. 137). RR.

- capitata Elirb. (Diat. d'Auv., p. 139). RR.

Actinella pliocenica F. Hérib. et M. Per. (Pl. VIII, fig. 7). - Longueur environ $100 \mu$, largeur de la plus grosse extrémité 10 à $12 \mu$; n'ayant pu observer de frustules entiers la petite extrémité nous est inconnue. Les bords de la valve ne portent pas de perles, mais seulement de petites granulations en nombre égal à celui des stries, et formées par leur prolongement. Stries transversales fines, non distinctement granulées, au nombre de 10 en $10 \mu$, et un peu plus serrées à la grosse extrémité. AC.

Notre Actinella ressemble i l'Actinella brasiliensis de Grunow. (V. H. Syn., pl. 35, fig. 19), espèce récente du 
Brésil; mais les deux espèces sont bien distinctes; elles diffèrent en effet par le nombre de leurs stries, et surtout par l'absence, sur la Diatomée de Celles, des perles nıarginales que l'on voit sur les bords de la valve de celle du Brésil.

Le genre Actinella, créé par Lewis, en 1881, ne comprenait encore que quatre ou cinq espèces toutes étrangères à l'Europe; deux appartiennent au Brésil, une à la Guyane et la quatrième à l'Amérique du Nord. Nous ignorons la patrie de l'Actinella scala, publié depuis peu, par M. Brun, professeur de Micrographie à l'Universitẻ de Genève. - Le genre Actinella est donc nouveau pour la flore européenne.

Asterionella antiqua F. Hérib. et M. Per. (Pl. VIII, fig. 8, sp. ?). - En comparant la fig. 8 de notre pl. VIII à la fig. 20 de la pl. 51 du Synopsis de $\cdot$ Van Heurck, il est bien évident que notre Diatomée est un Asterionella, mais le doute subsiste au point de vue de l'espèce, n'ayant pu observer un frustule entier. Largeur $7 \mu ; 12$ stries en $10 \mu$, interrompues par un pseudo-raphé assez large. $\mathrm{R}$.

Fragilaria brevistriata Grun. (Diat. d'Auv.,p. 146). AC.

- intermedic Grun. (Diat. d'Auv., p. 146). RR.

Tabellaria fenestrata Kttz. (Diat. d'Auv., p. 154). AR.

Tetracyclus costellatus (Ehrb.) nob. (Pl. VlII, fig. 12). = Biblarium costellatum Ehrb. RR.

Tetracyclus elegans (Ehrb., Microg.) nob. RR. - - var. eximia F. Hérib. et M. Per. (Pl. VlII, fig.15). - Sernblable au type, mais plus massif et á angles arrondis. Longueur $34 \mu$, largeur $27 \mu$. R.

Tetracyclus emarginatus (Ehrb.) nob. (Diat. d'Auv., p. 158). R.

— - var. crassa F. Hérib. et M. Per. (Pl. VIII, 
fig. 16). - Conformation générale du Tetracyclus cleguns (Ehrb.), mais à angles arrondis et plus massif. Longueur $40 \mu$, largeur $30 \mu$. AR.

Tetracyclus stella (Elırb.) nob. (Pl.VIII, fig. 9). R.

Tetracyclus Pagesi F. Héril). (Pl. VIII, fig. 10). De même forme que Tetrucyclus stclla (Ehrb.), mais deux fois plus grand, plus découpé et plus élégant. Longueur $50 \mu$, largeur $30 \mu$. R.

Cette jolie Diatomée est dédiée à M. J. Pagès-Allary, en souvenir de son aimable générosité.

Cymatopleur'a elliptica WV. Snı. (Diat. d'Auv., p. 160). R.

- - var. constricta Grun., p. 464, pl.11, fig. 13. R.

- Solea (Bréb.) IV. Sm. (Diat. d'Auv., p. 161). RR.

Nitzsclia sigmoidea Nitz. (Diat. d'Auv., p. 167). RR.

- Tabellaria Grun. (Diat. d'Auv., p. 166). RR.

Surirella noregica Ehrb. (Diat. d'Auv., p. 176). R.

- robusta Ehrb. (Diat. d'Auv., p. 180). AR.

Campylodiscus costatus W. Sm. (Diat. d'Auv., p.182).C. Melosira granulata Ehrb. (Diat. d'Auv., p. 186). CC.

- - var. arcuata F. Hérib. - Forme bien différente de la variété curvata de Grunow, intermédiaire entre les fig. 18 et 19, pl. 87 du Synopsis de Van Heurck. R.

- spiralis Ktz. V. II. Syn., pl. 87, fig. 19-22.

- - var. hemisphæerica M. Per. et F. Héril). (Pl. VIII, fig. 24 et 26). - Frustule composé d'une ralre plane ordinaire, et d'une valve hémisphérique, présentant, le plus souvent, une amorce á filament de plus petit diamètre. CC.

- - var. sphærica F. Hérib. et M. Per. (Pl. VIII, fig. 25). - Frustule complètement sphérique et sans anneau connectif ; Diatomée extrêmement curieuse. C.

Cyclotella Iris F. Hérib. (Diat. d'Auv., p. 221). CC.

- - var. integra M. Per. et F. Hérib. (Pl. VIII, fig. 31). - De forme presque toujours légèrement ellip- 
tique ou scutiforme, surtout chez les grands exemplaires; souvent strié symétriquement, non par rapport à un point central, mais par rapport au plus grand diamètre. Se distingue principalement du type par l'absence complète de centre hyalin. CC.

Le nombre des espèces ou variétés trourées dans le dépôt de Celles est de 64, parmi lesquelles 17 sont nouvelles pour la flore générale.

\section{Note additionele.}

Le tirage de la première feuille était fait, lorsque nous arons recu, de M. Pagès-Allary, un échantillon de la couche de terre noire sur laquelle repose le dépót de Celles. Nous nous sommes empressé de l'étudier avec le plus grand soin.

Après avoir constaté que la roche ne contenait pas d'éléments calcaires, nous en avons traité un fragment par $\mathrm{SO}^{4} \mathrm{H}^{2}$ bouillant, avec addition de $\mathrm{ClO}^{3} \mathrm{~K}$, dans le but de le rendre plus commode pour l'observation microscopique.

A la suite d'un examen très attentif, nous avons acquis la certitude que le produit, d'un blanc laiteux et très pur, de notre manipulation, ne renfermait nulle trace de Diatomées fossiles; par conséquent l'hypothèse d'un sol tourbeux n'est pas admissible, car tous les terrains de formation marécageuse contiennent toujour's des Diatomées plus ou moins abonduntes. A notre avis, la terre sur laquelle repose le dépôt de Celles est une couche d'argile, il éléments d'une extréme ténuité, et colorée en noir par un oxyde de fer. 


\section{DÉPOT DE LA BADE (Cantal)}

(Pliocène supérieur).

Le dépôt de la Bade a été trouvé par M. CharetonChaumeil, au mois d'aout 1900, et c'est par sa lettre du 19 octobre suivant qu'il nous annonca son intéressante décourcrte; en même temps, l'honorable avoué-géolog'ue de Langres eut l'amabilité de nous adresser un bel échantillon, nous permettant ainsi d'étudier le dépòt arant tout autre diatomiste.

Ce dépôt est situé au sud de Collandre, canton de Riom-ès-Montagne, près du hameau de la Bade, un peu au-dessus de la route, à l'altitude de 1,100 mètres. La dépression qu'il occupe actuellement devait aroir primitivement une longueur d'une centaine de mòtres, sur une largeur probable de 30 à 40 mètres ; sa plus grande épaisseur est de 8 mètres. Les trois quarts environ du volume ont déjà été enlevés par l'érosion, et le lambeau qui nous reste disparaîtra a son tour dans un arenir plus ou moins éloigné.

Au point de vue de son ige géologique, il appartient au pliocène supérieur; il se trouve, en effet, à la partie très supérieure des cinérites du pliocène, sous les basaltes du $\beta^{1}$; sa formation est done contemporaine des rastes dépòts d'Auxillac et de Celles.

A l'état sec, il est blanc ou jaune pille, pulvérulent et très pur. Les Diatomées sont en général très fragmentées, mème les espèces de petite taille, excepté les frustules de forme discoïde, comme les Melosira, les Cyclotella, etc.; cette fragmentation doit être le résultat de la pression 
énorme qu'a dủ subir le dépơt à l'époque de l'émission du basalte des plateaux.

La masse est très homogène, tant au point de vue des caractères physiques que sous le rapport de la florule diatomique; parmi les échantillons étudiés, et pris respectivement à la surface, ì 4 mètres et à 8 mètres de profondeur, nous n'avons pas, en effet, constaté de différence notable dans la liste des espèces observées.

La Diatomée caractéristique, et qui constitue à elle seule les neuf dixièmes du dépòt, est le Cyclotella Charetoni, que l'on peut rapporter, comme sous-espèce, au Cyclotella Iris des dépóts de Celles et d'Auxillac. Les autres espèces sont peu nombreuses et en exemplaires fort rares.

Dans les échantillons étudiés, nous avons trouvé les espèces et variétés suivantes :

Gomphonema intricatum Ktz. (Diat. d'Auv., p. 53). R. Amphora pediculus Grun. (Diat. d'Auv., p. 63). RR.

Gymbella Gharetoni F. Hẻrib. (Pl. VII, fig. 17). - De forme trapue, à extrémités largement arrondies et légèrement subrostrées. Longueur $85 \mu$, largeur $25 \mu$; raphé très légèrement biarqué, terminé par des nodules très petits; espace hyalin très large autour du nodule central, occupant plus de la moitie de la valve, et diminuant progressivement jusqu'aux nodules terminaux, ou les stries touchent le raphé. Stries fines, convergentes, non distinctement granulées, au nombre de 11 en $10 \mu$ a la partie dorsale, et de 12 en $10 \mu$ à la partie ventrale

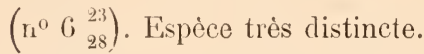

Diffère du Cymbella Ehrenbergii Ktz. par sa forme plus trapue, par son espace hyalin plus grand au milieu et plus étroit aux extrémités, par ses nodules terminaux plus petits et par ses stries plus serrées; dans Cymb. Ehrenbergii, on en compte seulement 8 en $10 \mu$. RR. 
Nous dédions cette espèce à M. Chareton-Chaumeil, en souvenir de sa découverte.

Cymbella Ehrenbergii Ktz. (Diat. d'Auv., p. 64). AIi. - turgidula Grun. (Diat. d'Auv., p. 68). RR. - lanceolata Ehrb. (Diat. d'Auv., p. 68). AR.

Navicula acrosphæria Bréb., var. badeana F. Hérib. et M. Per. (Pl. VII, fig. 2). - Se distingue du type par l'absence des points sablant le raphé et l'aréa; de la variété lcevis par la brièveté de ses cótes qui sont excessivement courtes jusque vers les renflements terminaux, et par la forme particulière du contour de l'aréa dans le voisinage des nodules terminaux. Longueur $150 \mu$, largeur $17 \mu ; S$ còtes très courtes en $10 \mu$. AR.

Navicula major Kitz. (Diat. d'Auv., p. \$2). R.

- radiosa Ktz. (Diat. d'Auv., p. 99). RR.

- - var. acuta Grun. (Diat. d'Auv., p. 99). RR.

- peregrina Ktz. (Diat. d'Auv., p. 100). RR.

- elliptica Kitz. (Diat. d'Auv., p. 101). R.

Epithemia Hyndmannii WV.Sm. (Diat. d'Auv., p.125).R.

- turgida Ktz. (Diat. d'Auv., p. 124). RR.

- gibba Ktz. (Diat. d'Auv., p. 126). AR.

- Zebra Ktz. (Diat. d'Auv., p. 127), AR.

-- - var. minor (Diat. d'Auv., p. 129). R.

Eunotia incisa Ehrb. (Diat. d'Auv., p. 133). Rli.

- polyglyphis Grun. (Diat. d'Auv., p. 134). R.

- lunar is Ehrb. (Diat. d'Auv., p. 135). RR.

Synedra pliocenica F. Ilérib. et M. Per. (Pl. VII, fig. 19). - Espèce très petite, en forme de losange allongé, á extrémités pointues et légèrement capitées; angles obtus. Longueur $26 \mu$, largeur $5 \mu$. Stries tres courtes, tout à fait marginales, au nombre de 12 en $10 \mu$. R.

Fragilaria lapponica Grun. (V. H. Syn., pl. 45, fig. 35). - Bien conforme a la fig. 35, pl. 45 du Synopsis 
de Van Heurck. Longueur $25 \mu$, largeur $\tau \mu$. Stries courtes, au nombre de 6 en $10 \mu . \Lambda R$.

- Surirella saxonica Auersw. (Diat. d'Auv., p. 176). RR.

Melosira undulata Kitz. (T. H. Syn., pl. 90, fig. 8-9). AR.

- - var. producta A. Sch. (Atlas, pl. 180, fig. S-13). R.

- arenaria Moor. (Diat. d'Auv., p. 186). AR.

Gyclotella Gharetoni F. Hérib. (Pl. VIII, fig. 30). - Très variable comme forme et comme grandeur; ordinairement elliptique plutòt que circulaire. Diamètre de 15 a $50 \mu$ et plus; valves fortement onclulées, couvertes de stries fines au nombre de 10 a 15 en $10 \mu$, suivant les dimensions des frustules, bifurquées d'une façon assez irrégulière, non dichotomes, mais formant plutòt des faisceaux irréguliers, laissant un centre lisse régulicr de grandeur moyenne. Face connective lisse, ne présentant qu'une rangée de ponctuations le long des bords qui sont á angles légèrement arrondis. Les points qui apparaissent sur la face connective sont formés par les extrémités des stries de la face valvaire.

Se distingue de notre Cyclotella Ir is type par les stries non dichotomes, par l'absence des points brillants que l'on voit aux bifurcations dichotomiques de la forme typique, par le centre plus régulier, plus petit et constamment lisse, tandis que celui de Cyclotella Iris est toujours sablé de ponctuations. L'ensemble de ces caractères diffèrentiels nous a paru suflisant pour séparer Cyclotella Charetoni de Cyclotella Iris, au moins à titre de sous-espèce. CC.

Cette Cyclotelle est dédiée a M. Chareton-Chaumeil, en souvenir reconnaissant de sa généreuse amabilité.

Gyclotella Gharetoni var. scutiformis F. Hérib. - Forme plus o 1 moins hiangulaire, analogue a celle du 
Cocconeis Pediculus; ordinairement plus robuste que le type. AC.

- var. radiata F. Hérib. et M. Per. - Toujours plus robuste que le type et de grande dimension; stries au nombre de 8 en $10 \mu$, ne présentant qu'exceptionnellement des bifurcations. C.

Nous avons trouvé, dans une préparation faite d'un échantillon appartenant à la zone moyenne, un fragment comportant le centre, deux portions de raphé, l'aire et une amorce des stries, lequel nous a paru ne pouvoir ètre assimilé à aucune espèce de Cymbella ou de Navicula connue de nous. Nous espérons trouver un autre fragment de valve plus grand, comportant une extrémité, de façon à pouvoir se prêter à une détermination au moins générique.

D'après la liste des Diatomées observées dans le dépót de la Bade, on voit qu'il est loin d'être aussi riche que celui de Celles. La pauvreté relative de la florule est due à l'étendue restreinte du dépòt, et surtout à sa masse trés lomogène. 


\section{DÉPOT DE PERRIER (Puy-de-Jòme)}

(Pliocène moyen).

C'est à M. Bouhard, chimiste-industriel à Paris, que nous devons communication de l'échantillon étudié. Le dépòt est situé sur le flanc sud de la montagne, immédiatement au-dessus des calcaires oligocènes; il est enchàssé dans des couches fluviatiles, composées de cailloux roulés, de sables et de cinérites argileuses.

La masse diatomifère est peu considérable, et il est de toute évidence qu'elle ne représente qu'un lambeau d'un dépòt formé à une altitude supérieure à celle de Perrier.

Les Diatomées sont assez nombreuses, mais tellement fragmentées et agglutinées que la détermination en est très difficile.

Parmi les nombreux débris nous avons pu reconnaitre les espèces suivantes :

Cocconeis lineata Grun. (Diat. d'Aur., p. 41). AC.

- Placentula Elirb. (Diat. d'Aur., p. 44). CC.

Gomphonema Kamtschaticum Grun. (V. H. Syn., pl. 25, fig. 29). - Absolument conforme à la figure citée du Synopsis de Van Heurck. RR.

Amphora affinis Ktz. (Diat. d'Auv., p. 63). RR.

Cymbella yastroides Kitz. (Diat. d'Auv., p. 68). AR.

- lanceolata Ehrb. (Diat. d'Auv., p. 75). CC.

Stauroneis gracilis WV. Sm. (Diat. d'Auv., p. 76). R.

- Phonicenteron Ehrls. (Diat. d'Aur., p. 75). AR.

Navicula Braunii Grun. (V. H. Syn., pl. 79, fig. 21). - Aréa stauronéiforme; nodule médian étroit ; longueur 35 a $10 \mu$; stries au nombre de $11 \mathrm{en} 10 \mu$. C. 
Nav. digito-radiata Greg., Micr. Journ. 1856, p. 9, pl. 1, fig. 32; V. H. Syn., p. 86, pl. 7, fig. 4. - De forme lancéolée, à extrémités arrondies; stries délicatoment granulées, au nombre de $\delta$ en $10 \mu$, un peu plus serrées aux extrémités. Longueur 60 à $68 \mu$, largeur 10 à $12 \mu$. R.

- gigas Ehrb. (Diat. d'Auv., p. 81). R.

- major Ktz. (Diat. d'Auv., p. 82). AC.

- gibba Ehrb. (Diat. d'Auv., p. 92). R.

- parva Grun. (Diat. d'Auv., p. 92). R.

- Bacillum Elırb. (Diat. d'Auv., p. 117). R.

- peregrina Heib. (Diat. d'Auv., p. 100). CC.

- - var. obtusa nov. - Forme plus courte, i extrémités plus massives, valve presque elliptique allongée; longueur $60 \mu$, largeur $16 \mu$; stries 7 en $10 \mu$. AR.

Navicula amphibola $\mathrm{Cl}$. var. perrieri M. Per. et F. Hérib. (Pl. VII, fig. 11). - Se distingue du type par sa forme plus trapue, par ses stries plus écartées, et dont quelques-unes sont terminées par un gros point situé près du nodule médian. Ressemble, comme forme et aspect général, à notre Navicula arverna (Diat. d'Auv., p. 105, pl. IV, fig. 19); en diffère par ses granules qui sont ronds au lieu d'ètre elliptiques, et par le raccourcissement des stries médianes, terminées par un gros point unilatéral. Longueur $60 \mu$, largeur $28 \mu$; stries au nombre de 6 en $10 \mu$.

Pantocseck donne, vol. III, pl. 22, fig. 340, une forme qui a aussi une certaine analogie avec la Diatomée de Perrier, et la nomme Navicula Moczarensis, mais il n'est pas possible de pouvoir identifier ces deux plantes. R.

Epithemia Hyndmannii WV. Sm. (Diat. d'Auv., p.125). C.

- Sorex Ktz. (Diat. d'Auv., p. 126). R.

- Zebra Ktz. (Diat. d'Auv., p. 127). R.

Synedra Ulna Elırb. (Diat. d'Auv., p. 137). AR.

L'examen d'autres échantillons permettrait probablement d'ajouter encore plusieurs formes intéressantes it celles de la liste précédente. 


\section{DÉPOT dU RaVIN DeS ÉGravaTS (Puy-de-Dòme)}

(Pliocène supérieur).

L'échantillon étudié provient des collections Bouillet, oì il figurait avec une étiquette portant un nom absolument étranger à la nature de l'objet; mais, l'indication du gisement étant très exacte, c'était pour nous le point essentiel.

Le ravin des Egravats est situé près de la Grande Cascade du Mont-Dore, á une altitude de 1,400 mètres; le dépót forme une assise ayant à peine 20 centimètres d'épaisseur, et se trouve immédiatement au-dessous d'une couche assez mince de lignite, surmontée d'une puissante formation de roches diverses, cinérites, trachyte, andésite.

A l'état sec, il est d'un blanc pur à la zone inférieure, et gris cendré à la partie supérieure; la florule de plusieurs échantillons étudiés ne présente pas de différence sensible, la masse est donc très lomogène. Par suite de la pression énorme produite par les roches supérieures, les Diatomées sont très fragmentées, mais très nettes et nullement agglutinées comme celles du dépót de Perricr.

Les espèces et variétés observées sont les suivantes:

Cocconeis Pediculus Ehrb. (Diat. d'Auv., p. 43). RR.

Gomphonema angustatum Grun. (Diat. d'Auv., p.60). R.

- - var. producta Grun. (Diat. d'Auv., p. 60). R.

- subcluvatum Grun. (Diat. d'Auv., p. 55). Ali.

('ymbella aspera Ktz. (Diat. d'Aur., p. 69). AC.

- maculata Ktz. (Diat. d'Auv., p. 71). R.

Navicula major Ktz. (Diat. d'Auv., p. \&\&). Ali.

- viridis Ktz. (Diat. d’Aur., p. 84). C.

- - var. commutata Grun. (Diat. d'Auv., p. 84). R. 
Navicula costata Ehrb. (Diat. d'Auv., p. 87). R.

- megaloptera Ehrb. (Diat. d'Auv., p. 88). RR.

- oblonga Ktz. (Diat. d'Auv., p. 98). C.

- elliptica Ktz. (Diat. d'Auv., p. 104). R.

Epithemia turgida Ktz. (Diat. d'Auv., p. 124). C.

- - var. granulati Grun. (Diat. d'Auv., p. 125). AC.

- - var. vertagus Ktz. (Diat. d'Auv., p. 125). AC.

- Zebra Kitz. (Diat d’Auv., p. 127). C.

Eunotia impressa Ehrb., Mik., pl. 15, fig. 56; Grun., 1862, p. 333; de Toni, p. 800 . RR.

Nous ne connaissions en Auvergne que la variété angusta de Grunow; le type est donc nouveau pour notre flore.

Au total, la florule de ce dépót est relativement paurre et peu intéressante. 

II.

\section{Revision de quelques Dépôts}

Il nous reste á donner le résultat de l'examen d'échantillons nouveaux des dépôts du Puy de Mur, de Verneuges, de La Bourboule, d'Auxillac et de Neussargues, étudiés trop sommairement en 1893.

\section{DÉPOT MARIN DU PUY DE NUR (Puy-de-Dòme)}

(Aquitanien).

Les échantillons examinés proviennent de deux affleurements nouveaux, que nous avons découverts l'un au N.-E., sur le talus ä'un chemin creux, non loin du domaine de Sainte-Marcelle, et l'autre au S.-O. ; la distance entre les deux points, situés à peu près au même niveau, est d'environ 1,800 mètres.

L'affleurement S., étudiẻ en 1893, ne nous a donné aucune forme nouvelle á ajouter à la liste des espèces et variétés déjá connues, tandis que celui de N.-E., très riche en Surirella Bruni et striatula; en Navicula aquitanice, recta, Julieni, bomboides et basaltaproxma, contient une belle variété de chacune des deux dernières Navicules, et, de plus, le genre marin Campylosir $a$, que nous n'avions pas encore trouvé dans ce curieux dépòt.

Obs. - Dans les listes des espèces et variétés observées dans les dépóts suivants, nous nous abstiendrons d'inscrire les formes mentionnées en 1893. 
Navicula bomboides A. Sch. var. limanense F. Hérib. (Pl. VII, fig. 15). - Se distingue du type par ses dimensions moindres, par l'étranglement médian moins accentué, par deux lignes entourant le raphé, laissant un espace parcouru par une rangée de petites perles rondes, en nombre égal á celui des stries. Longueur de la valve 75 à $80 \mu$, largeur 28 à $30 \mu$. Stries plus régulières et plus nettes, au nombre de 7 en $10 \mu$, formées de 4 à 6 grosses perles ovales. C.

Navicula bomboides A. Sch. var. minor F. Hérib. et Br. (Pl. VII, fig. 16). - Forme tout a fait analogue à la variété limanense, mais beaucoup plus petite. Longueur 45 a $50 \mu$, largeur 20 à $22 \mu$. AC.

Navicula basaltæproxima F. Hérib. et Br. var. longistriata M. Per. et F. Hérib. (Pl. VII, fig. 3). Diffère du type et de sa variété bigibba (Diat. d'Auv., p. 89), par les flancs de la valve presque rectilignes, et par les stries beaucoup plus longues surtout au milieu de la valve, où elles ne laissent qu'une aréa assez étroite des deux cótés du raphé, et simplement arrondie autour du nodule central. Longueur 85 i $90 \mu$, largeur $25 \mu$; largeur it l'étranglement de la valve $20 \mu$. Stries granulées, au nombre de 9 en $10 \mu$, et un peu plus serrées aux sommets de la valve. C.

Gampylosira Peragalli F. Hérib. (PI. VIII, fig. 17 à 19). - Valve de forme cymbelloïde, à extrémités récurvées du côté dorsal, sans être ni prolongées ni capitées; face connective ì centre et it extrénités élargis, présentant des ponctuations éparses et plus distinctes vers les bords. Frustules plus ou moins arqués et réunis en bandes. Longueur de la valve 40 à $45 \mu$, largeur de la région centrale 6 a $8 \mu$. AC.

Le genre Campylosira, crée par Grunow en 1882, ne comptait encore que deux espèces : Campylosira cym- 
belliformis Grun., assez fréquent sur les còtes de la Manche, et Campylosira japonica Temp. et Br., espèce récemment trouvée, par MM. Tempère et J. Brun, dans un dépôt marin du Japon.

La découverte de notre Campylosira Peragall vient confirmer, une fois de plus, l'origine marine du beau dépôt du Puy de Mur.

Nous sommes heureux de dédier cette Diatomée á M. le Commandant Maurice Peragallo, en souvenir de l'utile concours qu'il a eu l'amabilité de nous donner, dans l'étude des matériaux mis en ouvre pour la publication de ce mémoire.

C'est encore à titre de reconnaissance et de bon souvenir que nous arons tenu á joindre son nom au nòtre pour la plupart des formes nourelles.

Obs. - En comparant les florules respectives des trois affleurements connus du dépôt du Puy de Mur, nous constatons que celles du N.-E. et du S.-O. sont très riches en Surirella et Navicula de grande taille. Or, les diatomistes herborisants savent fort bien que ces Diatomées ne vivent que sur la vase, aux endroits où l'eau n'est pas assez profonde pour empècher l'accès de la lumière ; par conséquent leur présence indique, quand elles sont en grande abondance, les rivages de la dépression.

Dans l'affleurement S., au contraire, les Surirella et les Navicula sont très rares, tandis que les Melosira, les Cocconeis, les Fragilaria, les Striatella, les Periptera, les Raphoncis, les Coscinodiscus, etc., sont très abondants; ces petites Diatomées, connues sous la dénomination générale de Diatomées pélagiques, vivent et se multiplient à la surface des eaux profondes, caimes et ensoleillées; puis, de toute l'étendue de la surface elles se déposent au fond de la dépression, où elles s'accumulent peu ì peu pour former un dépòt plus ou moins consideirable, suivant la profondeur et la durée de la masse liquide. 
De la comparaison des trois florules, il résulte que les affleurements N.-E. et S.-O. indiquent les bords de la lagune, et celui du S. appartient á la partie profonde.

La présence des Diatomées d'eau douce, que l'on trouve mélangées aux espèces marines, doit être attribuée évidemment a un cours d'eau qui, à cette époque lointaine, devait se jeter dans la lagune; d'ailleurs le même fait so produit rle nos jours a l'embouchure de tous les fleuves, oir l'on observe un méiange de Diatomées d'eau douce et de Diatomées marines. 


\section{DÉPOT DE VERNEUGES (Puy-de-Dôme)}

(Quaternaire).

Le dépôt de Verneuges est situé à l'O. du lac d'Aydat, à une distance de 2 kilomètres et à 850 mètres d'altitude. L'échantillon nouveau, que nous devons à l'amabilité de M. Charles Saintigny, a été pris à 4 mètres de profondeur.

A l'état sec, il est d'un blanc grisàtre, pulvérulent et très pur. Nous y avons trouvé les espèces et variétés suivantes, que nous n'avions pas constatées dans l'échantillon très terreux étudié en 1890.

Gomphonema acuminatum Ehrb. var. gigantea F. Hérib. et M. Per. (Pl. VIII, fig. 2). -- Analogue à la forme grêle et élancée du type, mais beaucoup plus grand, atteignant jusqu'à $100 \mu$ de longueur, tandis que la longueur du type est à peine de $75 \mu$; largeur de la tête $18 \mu$. AR.

Gomphonema constrictum var. subcapitata Grun. (Diat. d'Auv., p. 52). AR.

Cymbella cuspidata Ktz. (Diat. d'Auv., p. 65). AC.

- anglica Lag. (Diat. d'Auv., p. 67). AR.

- cymbiformis Ehrb. (Diat. d'Auv., p. 69). R.

Navicula arverna F. Hérib. et M. Per. var. stauroneiformis M. Per. et F. Hérib. (PI. VII, fig. 10). Se distingue du type par la strie centrale qui n'est pas plus longue que la voisine, et s'arrête loin du nodule central ; les stries centrales, dont la décroissance est très curieuse et caractéristique, sont écourtées et simulent un stauros. Les points sont ronds, et les stries qui limitent le stauros 
ne sont pas terminées par un granule plus gros, comme dans le Nacicula amphibola Cleve. Le raphé est quelquefois un peu ondulé. Longueur de la valve $58 \mu$, largeur $26 \mu$. Cette variété sert de transition entre notre Navicula arverna et le Navicula amphibola Cleve. AC.

-- hybrida M. Per. et F. Hérib. (Diat. d'Auv.,p. 85). R.

- amphigomphus Ehrb. (Diat. d'Auv., p. 113). AR.

Navicula mesolepta Ehrb. var. Saintignyi F. Hérib. (Pl. VII, fig. 5). - Diffère du type par sa plus grande taille, par ses stries plus robustes et moins serrées, et surtout par la forme très particulière de son aréa qui, assez large autour du nodule central, diminue progressivement jusqu'aux nodules terminaux qui sont beaucoup plus gros que dans le type. R.

Nous dédions cette forme intéressante à M. Charles Saintigny, en souvenir des échantillons qu'il nous a procurés avec le plus aimable empressement.

Navicula viridis Ktz. forma anomala (Pl. VII, fig. 4). - Nous avons dessiné cette forme bizarre à titre de curiosité; d'ailleurs nous n'avons trouvé que cet exemplaire, et il est probable qu'il est unique dans le dépôt.

Synedra Ulna Ehrb. (Diat. d'Auv., p. 137). AC.

- capitata Ehrb. (Diat. d'Auv., p. 139). R.

Surirella elegans Ehrb. (Diat. d'Auv., p. 179). AR.

L'étude du dépòt de Verneuges reste incomplète, mais le temps nous manque pour la continuer sur de nouveaux échantillons. 


\section{DÉPOT DE LA BOURBOULE (Puy-de-Dòme)}

(Pliocène supérienr).

Le dépót de La Bourboule est aujourd'hui perdu pour les diatomistes, par suite de plusieurs constructions élevées sur son emplacement.

L'échantillon nouveau étudié provient des collections Bouillet.

Les Diatomées de ce dépôt sont peu variées; malgré le soin que nous avons mis à l'examiner, nous n'avons trouvé que deux espèces à ajouter à la liste publiée dans les Diatomées d'Auvergne, page 231, ce qui porte le nombre des espèces à 17 seulement, alors que d'autres dépôts, notamment ceux de Saint-Saturnin et d'Auxillac, en contiennent une centaine et plis.

Les deux espèces à ajouter sont :

Cocconeis Placentula Ehrb. (Diat. d'Auv., p. 44). R.

Navicula oblonga Ktz.(Diat. d'Auv., p. 9S). AR.

Le Cyclotella Temperei, espèce caractéristique du dépôt, n'ayant pas été dessiné pour les Diatomées d'Auvergne, nous donnons aujourd'hui une bonne figure de cette espèce remarquable, et nous complétons la description un peu sommaire de 1893.

Gyclotella Temperei M. Per. et F. Hérib. (Pl. VIII, fig. 23). - Diamètre de 12 à $25 \mu$; stries un peu ondulées, inégales; striation analogue à celle du Cladogramma cebuense Grun. de l'île Cebu (Philippines). Marge portant une rangée de perles; aréa parfois presque nulle, sablée 
de granules peu visibles. L'exemplaire dessiné représente l'une des plus grandes formes. CC, formant presque toute la masse du dépôt.

Ce Cyclotella est dédié à M. J. Tempère, le micrographe préparateur bien connu des diatomistes.

Le dépòt remanié de La Bourboule, très riche en végétaux fossiles d'une conservation merveilleuse, mérite d'ètre recherché dans le voisinage des constructions qui nous ont dérobé l'affleurement découvert par Lecoq et Bouillet. 


\section{DÉPOT D'AUXILLAC (Cantal)}

(Pliocène supérieur).

L'impression des Diatomées d'Auvergne étant presque terminée à l'époque de la découverte (1893) du dépòt d'Auxillac, près de Murat, il ne nous fut pas possible de l'étudier avec toute l'attention voulue; pressé par le temps, il fallut nous borner à l'examen rapide d'un petit nombre d'échantillons, et remettre á plus tard le soin de compléter nos premières recherches.

Voici le résultat de l'ètude de plusieurs échantillons nouveaux :

Cocconeis lineata Grun. (Diat. d'Auv., p. 44). AR.

- - var. euglypta Grun. (Diat. d'Auv., p. 46). R.

Rhoicosphenia curvata Grun. (Diat. d'Auv., p. 51). RR.

Gomphonema cantalicum F. Hérib. et Br., rar. lepida F. Hérib. et M. Per. (Pl. VIII, fig. 1). - Diffère du type par ses nodules, et par son point unilatéral plus petit et placé plus près des stries, qui sont plus fines, très faiblement granulées, et ne sont point coupées par une ligne d'interruption. Longueur de lia valve 150 à $200 \mu$, largeur vers le nodule central $2 \mathcal{S} \mu$; stries au nombre de 12 en $10 \mu$. R.

Gomphonema subclavatum Grun. (Diat. d'Auv.,p.55).R. Cymbella cuspidata Ktz. (Diat. d'Auv., p. 65). RR.

- parva W. Sm. (Diat. d'Auv., p. 70). R.

- maculata Ktz. (Diat. d'Aur., p. 71). K.

Navicula amphibola $\mathrm{Cl}$. var. stauroneiformis M. Per. et F. Hérib. (Pl. VII, fig. 12). - Longueur de li 
valve $65 \mu$, largeur $22 \mu$; stries convergentes, formées de granules petits et distincts; les centrales fortement et brusquement écourtées d'environ la moitiẻ de leur longueur, et formant ainsi un stauros; de plus, elles sont moins serrées au centre $(6$ en $10 \mu)$ qu'aux extrémités (8 en $10 \mu)$. R.

Cleve a isolé, avec raison, le Navicula styriaca Grun. pour en former son Nat. amphibola, laissant le nom de Nav. styriaca au Ian Heurckia styriaca Grun.

Nous arons adopté cette manière de roir.

Navicula Renauldi F. Hérib. (Pl. VII, fig. 9). De forme elliptique, à extrémités fortement rostrẻes et légèrement capitées. Raphé fin ; nodules centraux et terminaux petits. Longueur $35 \mu$, largeur $13 \mu$. Stries convergentes, courbes, normales á la fois au bord de la valve et au raphé qu'elles arrivent à toucher, laissant au centre une aréa très petite, nettement granulées, au nombre de 10 en $10 \mu$ au centre, et un peu plus serrées aux extrémités. Espèce très distincte. R.

IValle représente, pl. 19, fig. 29, sous le nom de Navicula inflata Ktz., une forme assez semblable à notre Navicula Renauldi, mais il reproduit, en se trompant évidemment de nom, la figure du Navicula tumida IV. Sm., pl. 17, fig. 146. Le Navicula inflata Ktz. est tout a fait différent, et quant au Navicula tumida W. Sm., Cleve l'assimile au Navicula anglica Ralfs, qui possède une aréa centrale assez grande qui n'existe pas ici.

Nous dédions cette jolie Navicule à notre éminent ami et savant bryologue, M. le Commandant F. Renauld.

Pleurosigma attenuatum Ktz. (Diat.d'Auv.,p. 122). Rl.

Epithemia gibla var. ventricosa (Diat. d'Auv., p. 126). C.

- Zebra Ktz. (Diat. d’Auv., p. 127). C.

Synedra closterioides Grun. var. fossilis M. Per. et F. Hérib. (Pl. VIII, fig. 5 et 6). - Longueur de la 
valve 60 a $80 \mu$, présentant un renflement central visible tant sur la face valvaire que sur la face connective. Stries marginales et très fines, au nombre de 14 en $10 \mu$ au centre et de 18 en $10 \mu$ aux extrémites, qui sont ordinairement recourbées dans le même sens. - Diffère de la forme type (V.H. Syn., pl. 70, fig. 10 et 11) en ce que la partie élargie est plus courte et les rostres proportionnellement plus longs. CC.

Flagilaria mutabilis Grun. (Diat. d'Auv., p. 145). AC.

Tetracyclus costellatus (Ehrb.) var.turris M.Per. et F. Hérib. (Pl. VIII, fig. 13), - Ressemble au Biblarium costellatum Ehrb., mais, au lieu de présenter un contou général elliptique, il est ici circulaire. Longueur dı diamètre $20 \mu$. RR.

L'échantillon observé et dessiné ne portait que l'anneau connectif.

Tetracyclus ellepticus (Ehrb.) var. minutissima F. Hérib. et M. Per. - Très petit, ayant à peine $13 \mu$ de longueur. Pourrait être pris par les débutants pour un frustule de Diatoma hyemale Heib. ou pour un Diatoma Mesodon Ktz. R.

Tetracyclus tripartitus var. gracilis M. Per. et F. Hérib. (Pl. VIII, fig. 11). - Tout à fait semblable à notre Tetracyclus tripartitus (Diat. d'Aur., pl. 6, fig. 5), mais moins robuste, présentant des côtes non interron1pues et des ondulations très peu sensibles. Longueur $80 \mu$, largeur du renflement médian $15 \mu$. RR.

- lancea (Ehrb.) nob. (Diat. d'Auv., p. 159). R.

- rhombus Ralfs (Diat. d'Auv., p. 160). AC.

Cymatopleura Solea IV.Sm. (Diat. d'Auv., p. 161). RR.

Melosira Sol (Ehrb.) Ktz. = Gaillonella Sol Ehrb. Le Melosira d'Auxillac est tout à fait identique à celui des côtes occidentales de l'Amérique, et figuré par Tan IIeurck Syn. pl. 91, fig. 8 et 9. R. 
Melosira tenuissima Grun. (Diat. d'Auv., p. 188). C'. Cyclotella Kutzingiana Ch. (Diat. d'Auv., p. 192). R.

Cette Cyclotelle était déjå connue en Aurergne í l'état vivant; il est intéressant de savoir qu'elle existait aussi it l'époque lointaine du pliocène supérieur.

La florule du dépót remanié d'Auxillac comprend une centaine d'espèces ou variétés, parmi lesquelles une quarantaine sont inédites et très remarquables. 


\section{DÉPOT DE NEUSSARGUES (Cantal)}

(Miocène).

Il ne nous reste aujourd'hui, du dépòt de Neussargues, que des lambeaux disséminés çà et lá dans la vallée et respectés par l'érosion; celui de Joursac est à la fois le plus important et le plus intéressant, á cause des végétaux fossiles qu'il contient en plus grande abondance.

Comme tous les dépòts diatomifères qui contiennent des empreintes de feuilles, celui de Neussargues a été évidemment remanié; les lambeaux que nous étudions aujourd'hui sont des débris entraînés d'un dépót, probablement très vaste, formé à une altitude supérieure à celle de Neussargues.

Les échantillons étudiés proviennent d'un lambeau découvert par nous au mois d'aoùt 1895, et situé sur le bord de la route, entre la gare et l'Allagnon.

Les espèces et variétés observées sont les suivantes :

Navicula sculpta Ehrb. = Nav.tumens W. Sm. Bien conforme à la fig. 1, pl. 12 du Synopsis de Van Heurck. Longueur 75 i $80 \mu$, largeur 20 a $25 \mu$. Stries granulées, interrompues près du raphé, au nombre de 15 en $10 \mu$. R.

Navicula slesvicensis Grun. (Diat. d'Auv., p. 101). R.

Navicula Dariana A. Sch. var. miocenica F. Hérib. et M. Per. (Pl. VII, fig. S). — De forme lancéolée, à extrémités largement arrondies. Aréa large, arrondie autour du nodule central, et diminuant progressivement jusqu'aux nodules terminaux qui sont grands et ronds. 
Longueur $150 \mu$, largeur vers le nodule central $32 \mu$. Stries lisses, radiantes au centre, au nombre de 6 en $10 \mu$, puis divergentes aux extrémités, ou elles sont au nombre de 7 en $10 \mu$. R.

Schmidt assimile Nuvicula Dariana au Pinnularia Porrecta d'Ehrenberg, en faisant observer toutefois que le Pinnularia Porrecta ne doit être qu'une forme particulière du Cymbella Ehrenbergii Greg. - Nous ne connaissons pas le dessin d'Ehrenberg, mais nous savons qu'il a été fait d'après une espèce du dépôt de Santa-Fiora. Or, dans une préparation de ce dépôt, que possède M. le Commandant Maurice Peragallo, on observe de nombreux exemplaires de Cymbella Ehrenbergii dont quelques-uns sont presque symétriques, mais, dans ce cas, la forme qui en résulte n'a pas le même contour que celui du Navicula Dariana, il dérive de la forme générale du Cymbella Ehrenbergii, c'est-à-dire qu'il est plus elliptique, avec des extrémités franchement atténuées et subrostrées.

Navicula Bouhardi F. Hérib. (Pl. VII, fig. 1). La conformation générale est celle du Navicula cuspidata Ktz., dont il se distingue par les extrémités qui rappellent celles du Nav. anebigua Ehrb., par les nodules centraux plus gros et entourés d'une aréa plus large, par ses dimensions plus grandes. Longueur 120 ì $130 \mu$, largeur 30 à $40 \mu$. Stries fines, au nombre de 16 en $10 \mu$. Silice hyaline et délicate. AC.

Nous dédions cette espèce à M. Bouhard, chimisteindustriel a Paris, en souvenir des échantillons qu'il a eu la bonté de nous procurer.

Navicula Malinvaudi F. Hérib. (Pl. VII, fig. 6). - Cette belle espèce appartient au groupe des Sculptées; elle est intermédiaire entre les Navicula sculpta et bohemica d'Ehrenberg. Les stries sont formées par des granules épars sur leur longueur, assez éloignés les uns des autres, et formant avec ceux des stries adjacentes des 
lignes interrompues fortement et irrégulièrement ondulées ; sur les bords de la valve les stries sont indiquées par des points bien marqués, et elles s'atténuent progressivement vers le raphé, où elles se terminent par un point bien net; tous ces points serrés les uns contre les autres forment quatre lignes d'aspect légèrement lyré, qui accompagnent le raphé. Les points formant les stries, tout en s'atténuant, ne disparaissent pas complètement comme dans le Navicula sculpta, de sorte qu'il n'y a pas d'aires latérales lisses entre la ligne des points avoisinant le raphé et les stries marginales. Sur l'un des còtés, l'atténuation des stries est un peu plus marquée sur une bande partant du nodule central et allant vers le bord de la valve. Longueur du frustule $90 \mu$, largeur $25 \mu$; stries au nombre de 18 en $10 \mu$. - Espèce très distincte.

Cette jolie Navicule est dédiée à notre vieil ami, M. Ernest Malinvaud, le très sympathique et savant Secrétaire général de la Société botanique de France.

Eunotia gracilis Rab. var. capitata M. Per. et F. Hérib. (Pl. VIII, fig. 21). - Diffère principalement du type, en ce que ses extrémités, au lieu d'ètre simplement récurvées sur la face dorsale, sont sensiblement élargies en forme de tête ronde et légèrement récurvées. Valve assez fortement courbée, parfois même genouillée. Longueur environ $80 \mu$, largeur $5 \mu$; stries au nombre de 11 en $10 \mu$. R.

Opephora Martyi F. Hérib. (Pl. VIII, fig. 20). Diatomée de forme ovale, très petite. Longueur $17 \mu$, la plus grande largeur $7 \mu$. Stries larges, lisses, au nombre de 6 en $10 \mu$, ne laissant au centre qu'une ligne très étroite.

L'Opephor'a Mar'tyi ne peut ètre confondu avec certaines formes plus ou moins coniques du Fragilaria brevistriata Grun., dont les stries, beancoup plus courtes et moins larges, laissent une aréa large et lisse. Il n'est pas possible non plus de l'assimiler à l'Opephora Schwartìii Petit, espèce marine vivante des îles Carolines, beaucoup plus 
grande (longueur 60 à ' $72 \mu$ ) et les côtes plus grosses (4 en $10 \mu$ ), laissant au centre uné ligne lisse plus large. Notre Opephora ressemble davantage à l'Opephora pacifica Petit du Brésil, mais la Diatomée de l'Amérique du Sud est marine et plus grande (longueur $40 \mu$ ), les côtes relativement moins larges et plus serrées ( 8 en $10 \mu$ ), laissant une ligne médiane lisse et large, allant en s'élargissant du petit bout du frustule à l'autre extrémité. - En résumé, l'Opephora Martyi est une espèce très distincte. R.

Nous dédions cette Diatomée à notre aimable et distingué compatriote, M. Pierre Marty, en souvenir des éléments d'étude qu'il a eu l'amabilité de nous procurer. C'est dans un échantillon de Neussargues, reçu de notre cher paléobotanistc cantalien, que nous avons eu le plaisir de la découvrir.

Le genre Opephora Petit, de création récente, ne comprenait encore que les deux espèces que nous venons de nommer, l'une des Carolines et l'autre du Brésil. Il est instructif de constater que dès l'époque tertiaire Opephora Martyi vivait chez nous, à côté de l'Actinella pliocenica, au temps où Torreya nucifera, Lilia expansa, Laurus canariensis, Pterocarya fraxinifolia, Grevia crenata, Sassafras Ferretianum, Zelkova crenata, etc., des cinérites de la Mougudo, près de Vic-sur-Cère, épanouissaient leurs fleurs et mûrissaient leurs fruits sous le climat brésilien de ces âges lointains.

Surirella biseriata Bréb. (Diat. d'Auv., p. 177). AR.

- tenera Greg. (Diat. d'Auv., p. 180). R.

- - var. splendidula Greg.

Melosira Boulayana M. Per. (Pl. VIII, fig. 27 et 28). AR. - Valves dissemblables; la supérieure porte à la circonférence une garniture de côtes doubles en forme de plis formés par deux stries; ces côtes, au nombre de 5 ou 6 en $10 \mu$, sont écartées les unes des autres d'environ une largeur de strie, ou la moitié de leur épaisseur. La 
valve inférieure porte aussi une garniture de côtes robustes et écartées les unes des autres d'une distance à peu près égale á la moitié de leur épaisseur, et elles sont couronnées par une perle qui fait suite à la còte et se projette en dehors de la valve comme un point sur un $i$, de manière á lui donner un aspect crénelé. Sur les deux valves, les cótes s'affaiblissent progressivement et s'évanouissent vers les deux tiers du rayon, laissant le centre lisse. A 5 ou $6 \mu$ de la circonférence, les stries s'infléchissent et paraissent plus marquées á partir de cette région. Cette courbure des stries fait que le centre n'est pas au niveau des bords.

Quand les valves sont accolées, les còtes ou les perles de la valve inférieure viennent s'intercaler entre les côtes doubles de la valve supérieure. Diamètre 40 ì $55 \mu$, côtes doubles au nombre de 5 en $10 \mu$. Espèce bien distincte et très remarquable, n'ayant d'analogie avec aucune autre espèce connue.

Ce Melosir $a$, dédié au savant doyen des Facultés catholiques de Lille, M. l'abbé Boulay, a étẻ d'abord découvert par M. le Commandant Maurice Peragallo, dans le dépòt de Ranc (Ardèche); il est assez fréquent dans celui de Neussargues.

Dans un échantillon, nous avons trouvé de nombreux fragments de Surirella on de Coscinodiscus qui paraissent nouveaux, ou tout au'moins très intéressants ; malheureusement leur état de fragmentation ne nous a pas permis de les étudier. Il serait utile de traiter une assez grande partie de cet échantilion par des méthodes aussi délicates que possible, de façon à avoir des frustules entiers, ou des fragments plus complets. 


\section{RÉSULTATS ACQUIS.}

En totalisant les Diatomées observées dans les dépòts étudiés pour la publication de ce mémoire, nous trouvons qu'elles sont au nombre de 160 ; et, on négligeant 93 espèces dẻjá mentionnées dans les Diatomées d'Auvergne, il nous en reste 67 à ajouter à notre flore diatomique, parmi lesquelles les formes suivantes sont inédites :

Gomphonema biventralis.......... Dépôt de Celles.

- acuminata var. gigantea....... - Verneuges.

- insigne var. acuminata......... - Celles.

- subclavatum var. major........ - id.

- cantalicum var. lepida......... - Auxillac.

Cymbella Charetont.............. - La Bade.

Encyonema Girodi.............. - Celles.

Navicula cellesensis............. - id.

- acrospheria var. badeana...... - La Bade.

- Dariana var. miocenica........ - Neussargues.

- Gomontiana................ - Celles.

- Pagesi.................... - id.

- Malinvaudi................ - Neussargues.

- mesolepta var. Saintignyi....... - Verneuges.

- arverna var. stauroneiformis.... - id.

- viridis forma anomala......... - id.

- amphibola var. perrieri........ - Perrier.

- - var. stauroneiformis....... - Auxillac.

- bomboides var. limanense...... - Puy de Mur.

- peregrina var. obtusa.......... - Perrier.

- Renauldi.................. - Auxillac.

- basaltceproxima var. longistriata. - Puy de Mur. 
Actinella pliocenica............. Dépòt de Celles. Eunotia gracilis var. capitata..... - Neussargues. Asterionella antiqua............. - Celles.

Opephora Martyi............... - Neussargues.

Campylosira Peragalli.......... - Puy de Mur.

Synedra closterioides var. fossilis... - Auxillac.

- pliocenica................. - La Bade.

Tetracyclus costellatus var. turris.. - Auxillac.

- clegans var. eximia........... - Celles.

- emarginatus var. crassa...... - id.

- Pagesi.................... - id.

- ellipticus var. minutissima...... - Auxillac.

- tripartitus var. gracilis........ - id.

Melosira granulata var. arcuata... - Celles.

- spiralis.................. - id.

- - var. hemispherica........ - id.

- - var. splecerica............ - id.

Cyclotella Charetoni............ - La Bade.

- - var. scutiformis........... - id.

- - var. radiata............. - id.

- Iris var. integra............ - Celles.

Soit un total de 43 espèces ou variétés nouvelles pour la flore générale. 


\section{OBSERVATIONS SUR LES DÉPOTS A DIATOMÉES}

Un dépôt à Diatomées n'est autre chose, en réalité, que la vase accumulée au fond d'une masse d'eau profonde d'une étendue plus ou moins considérable.

Les dépóts ne se différencient que par leur âge géologique et par la nature de l'eau dans laquelle ils se sont formés. Qu'ils soient marins, saumâtres ou d'eau douce, le mode de formation est identique pour tous.

Dans tous les cas, les Diatomées exigent pour vivre et se multiplier, une eau pure et éclairée ; ces petites Algues ne se développent jamais dans les eaux corrompues ou bourbeuses, ni dans l'obscurité absolue.

Une nappe d'eau profonde, limpide et ensoleillée, comme le sont les lacs d'Auvergne, est particulièrement favorable au développement de ces microorganismes.

D'une façon générale, on peut dire que deux catégories de Diatomées concourent à la formation d'un dépôt : le premier groupe comprend les grandes espèces appartenant aux genres Pinnularia (Navicula pr. p.), Surirella, Cymatopleura, Pleurosigma, etc; ces Diatomées se développent exclusivement sur la vase des fossés, des mares, des étangs, des lacs; par conséquent, dans les lacs peu profonds, comme ceux d'Aydat (15 mètres), de Guéry (8 mètres), du Chambon ( 6 mètres), de Chambedaze (5 mètres), des Esclauzes (4 métres), etc., elles pourront vivre et se propager sur toute la surface du fond ou l'éclairement, quoique faible, du moins pour certains points du lac d'Aydat, suffit à leur développement normal.

Dans les lacs plus profonds, comme le lac Pavin (95 mètres), le lac Chauvet (64 mètres), le gour de Taze- 
nat (67 mètres), etc., il y a lieu de tenir compte de la limite de l'obscurité physiologique, laquelle varie évidemment suivant la diaphanéité ou la transparence de l'eau; les lacs d'Auvergne, situés presque tous dans la région montagneuse, étant d'une très grande limpidité, cette limite n'est atteinte qu'á une vingtaine de mètres, tandis que pour les lacs de la plaine elle ne descend guère audessous de 15 mètres (1). Dans ces conditions, notre premier groupe de Diatomées ne pourra se développer qu'aux bords du lac, sur une zone plus ou moins large, suivant la déclivité du sol, et la vie des petites Algues cessera dès que la profondeur de l'eau ne permettra plus l'accès de la lumière.

Le second groupe, beaucoup plus important, comprend un grand nombre d'espèces de taille minuscule, dont les frustules sont accolés en rubans ou en tubes par leur face valvaire. Elles appartiennent presque exclusivement aux genres Melosira, Cyclotella, Meridion, Himantidium, Achnanthes, Fragilaria et Tabellaria, et nous sont déjà connues sous le nom de Diatomées pélagiques; au lieu de se développer sur la vase, comme celles du premier groupe, elles vivent et se multiplient, au contraire, a la surface de l'eau et dans les zones très supérieures fortement éclairées, oì elles sont mélangées aux organismes nombreux qui forment la faune inférieure du lac. Les limnologistes ont donné à l'ensemble de ces organismes, y compris les Diatomées, le nom de plankton. On sait avec quelle étonnante rapidité le plankton d'un lac se transforme selon la saison, l'état de l'atmosphère et l'heure du jour. Ce curieux phénomène a attiré l'atten-

(1) M. Husnot, dans son Muscologia gallica, page 348, nous dit lien que le Thamnium alopecurum var. lemani Schut. a été cueilli par M. Guinet, dans le lac de Genève, à 60 mètres de profondeur; mais, c'est là évidemment une erreur typographique; à cette profondeır, en effet, ainsi que nous l'avons fait observer dans nos Muscinées d'Auvergne, page 233, le développement d'une plante à clloroplyylle n'est pas possible. 
tion de quelques observateurs, notamment du professeur Cleve, de l'Université d'Upsal, de M. le professeur $\mathrm{D}^{\mathrm{r}}$ Paul Girod et de nos distingués collègues, MM. Ch. Bruyant et A. Eusébio. L'étude méthodique des planktons des lacs d'Auvergne est assurément des plus fécondes en découvertes, et pent nous donner la clef de bien des faits encore mal élucidés.

Quelle que soit la transformation du plankton, les Diatomées en constituent toujours, par leur abondance, la fraction prédominante, en particulier pendant les trois premières saisons de l'année.

Ces Algues microscopiques se multiplient surtout par déduplication, avec une rapidité telle que de tous les points de la surface, et i tout instant, les frustules adultes descendent au fond du lac par légions innombrables; un temps chaud et orageux active leur multiplication.

La vase n'est pas formée uniquement de carapaces siliceuses de Diatomées ; des masses plus ou moins considérables de feuilles, de sable, de scories légères, apportées par le vent, se déposent dans le lac et vont s'accumuler au fond en s'ajoutant aux Diatomées; les lacs entourés de bois comme le lac Pavin, le lac Servière, le lac Chambon, etc., recoivent surtout de nombreuses feuilles, mais il est a noter que ces feuilles se décomposent très rapidement; leur conservation dans la vase diatomifère du fond ne saurait être de longue durée, et elles ne laisseront nulle trace dans le dépốt. D'ailleurs, les dépóts quaternaires de Ceyssat, de Randanne, de Vassivière, de Ponteix, etc., sont absolument dépourrus d'empreintes végétales, et pourtant des feuilles innombrables se déposèrent aussi au fond des lacs dans lesquels se sont formés ces dépôts à Diatomées ; d'ou il suit que la fossilisation des feuilles n'est pas possible au cours de la formation d'un dépôt diatomifère; nous insistons sur ce point, en raison de son importance pour les résultats de nos observations.

De l'accumulation des Diatomées et de tous les objets 
légers apportés par les courants aériens, il résulte que le fond du lac s'exhausse progressivement d'une façon très lente, mais continue. La dépression occupée par la masse liquide finira donc par être complètement comblée par le dépôt; à un certain moment, la profondeur peu considérable de l'eau permettra aux plantes hydrophiles de s'établir sur la surface boueuse du dépôt, ainsi que nous le constatons actuellement sur les anciens lacs de la Cassière et d'Espinasse; les détritus de cette végétation très vigoureuse produiront une couche végétale de plusieurs mètres d'épaisseur, comme celle qui recouvre aujourd'hui le dépôt de Verneuges; puis la masse diatomifère se dessèchera plus ou moins par évalporation, prendra une coutleur gris clair ou cendré, et la province comptera un dépôt à Diatomées de plus, mais un lac de moins.

Tous les beaux lacs d'Auvergne sont ainsi condamnés à disparaître successivement dans un avenir plus ou moins éloigné; déjà la profondeur de plusieurs est très faible, comme celle du lac inférieur de la Godivelle, du lac des Esclauzes, de Chambedaze, etc. Le lac Pavin sera très probablement le dernier survivant, á cause de sa grande profondeur actuelle et de la pureté de son dépót. L'étude d'un échantillon pris à 95 mètres nous a permis de constater que la vase de ce lac contient environ $90 \%$ de valves siliceuses de Diatomées, alors que celle du lac d'Aydat en renferme à peine $60 \%$. A notre avis, cette différence énorme doit être attribuée uniquement à la situation topographique des deux lacs; le lac Pavin, entouré de pàturages et èloigné de terres cultivées, ne reçoit que très peu d'éléments terreux, tandis que le lac d'Aydat, situé à proximitẻ de champs cultivés et de sommets dénudés, reçoit de très grandes quantités de poussières et de sables volcaniques apportées par le vent. Ce dépòt est donc relativement terreux, comparé à celui du lac Pavin, et, par suite, de formation plus rapide.

Les détails que nous venons de donner sur la formation 
d'un dépòt quaternaire, s'appliquent à tous les dépóts à Diatomées en général.

Il nous reste maintenant á examiner les dépòts diatomifères qui renferment des empreintes de feuilles.

A propos des dépóts de Celles, de Neussargues et de Joursac, nous avons fait observer qu'ils ne s'étaient pas formés á la place où nous les trouvons actuellement, et que, de plus, la florule des plantes fossiles (florule phanérogamique) contenues dans la masse á Diatomées, doit être postérieure à la florule diatomique, parce qu'elle n'a pu se former qu'i l'époque où les dépỏts ont été remaniés.

Nous aurions pu ajouter qu'il doit en ètre ainsi pour tous les dépóts diatomifères rentermant des empreintes de feuilles; c'est une loi générale ne comportant aucune exception.

Dans certains cas particuliers, les deux florules, bien loin d'ètre synchroniques, peuvent même appartenir à deux époques géologiques différentes. Tel est le cas, par exemple, du dépòt de Saint-Saturnin (Puy-de-Dòme), dont la florule phanérogamique, étudiée par l'abbé Boulay, est franchement quaternaire; elle ne comprend, en elfet, que les feuilles des végétaux ligneux, arbres et arbustes, qui peuplent et décorent de nos jours la belle vallée de la Monne. La florule diatomique, au contraire, présente non seulement une physionomie d'antiquité que n'ont pas les dépòts quaternaires, mais encore une série nombreuse de Diatomées caractéristiques des dépôts du pliocène supérieur, telles que : Achnanthes subsessilis, Gomplonema tergestina, Navicula aponina et minuscula, Fragilaria bidens, Diatoma elongatum, Cymatopleura hibernica, Nitzschia norvegica et turgida, Melosira tenuissina, Cyclotella Meneghiniana, Stephanodiscus Astreca, etc. La florule diatomique de ce dépôt se rapporte donc bien au pliocéne supérieur, et la florule phanérogamique au quaternaire.

Le dépòt ne s'est pas formé à la place où nous l'étudions 
aujourd'hui, car les feuilles très abondantes, et d'une conservation parfaite, que l'on trouve dans toute son épaisseur, n'ont pu se fossiliser. au cours de la formation très lente et lacustre de la masse diatomifère; on sait, en effet, que la conservation d'un objet délicat exige qu'il soit recouvert très rapidement, afin de le soustraire aux causes multiples de la décomposition ; or, cette condition essentielle ne saurait être réalisée pendant la formation d'un dépòt à Diatomées, ainsi que nous venons de le voir.

Le dépòt s'est done formé á une distance plus ou moins grande du point où il est actuellement; puis, à la suite des commotions qui ébranlèrent le sol, à l'époque des éruptions volcaniques quaternaires, auxquelles nous devons la chaîne classique des monts Dòmes, il fut repris par les eaux et entrainé, sous forme d'un courant boueux, vers la dépression qu'il occupe maintenant.

Pendant que la boue diatomifère se déposait au fond de la dépression, des objets légers, tels que des feuilles d'arbres, des graines appendiculées, des insectes, etc., poussés par le vent s'y abattaient péle-mêle, et s'ajoutaient aux objets de même nature charriés par le courant; tous ces objets délicats étaient successivement et rapidement recouverts par l'arrivée de nourelles quantités de dépôt. Selon les lieux et à certains moments, des sables, des scories légères, des cendres volcaniques venaient s'intercaler dans la masse diatomifère, ainsi que nous l'avons fait observer á propos du dépòt de Celles.

" Enfin la lave qui, figée, constitue le basalte, est venue comprimer le tout de sa masse et do son poids énorme, expulsant l'eau et les gaz et assurant pour des centaines de siècles la conservation parfaite d'objets parfois merveilleusement délicats (abbé Boulay) ". Nos dépòts tertiaires sont en effet recouverts par des roches d'origine volcanique, plus rarement par des accumulations morainiques (dépót de Celles).

Tous ces phénomènes, très analogues à ceus qui ont 
concouru à la formation des bassins houillers, se passèrent évidemment durant une période de calme relatif, et dins un laps de temps restreint.

Mais, comme le remaniement s'est effectué, pour chaque dépôt, dans des conditions particulières et très diverses d'ordre local, que nous ne pouvons détailler ici, il en est résulté que certains dépóts, tels que ceux d'Auxillac, de Neussargues, de Celles, etc., ne contiennent que très peu de végétaux supérieurs fossiles, contrairement á ceux de Saint-Saturnin, de Varenne, de La Bourboule, etc.

Le dépôt marin du Puy de Mur n'a pas été déplacé ; la présence des poissons fossiles qu'il renferme et l'absence d'empreintes de feuilles ne permettent pas d'admettre un remaniement de la masse diatomifère.

Quant aux dépóts à plantes fossiles qui ne contiennent pas de Diatomées, tels que ceux de Menat et de Gergovie (Puy-de-Dóme), de la Mougudo, de Saint-Vincent et de Niac (Cantal), il est évident qu'ils se sont formés á la place où nous les trouvons aujourd'hui, et leur mode de fossilisation ne diffère de celui de la florule quaternaire de Saint-Saturnin que par la nature du sédiment : au lieu d'un sédiment diatomifère nous avons, à Gergovie et à Niac, une argile fine, d'un gris clair ou cendré, à Menat, du tripoli composé d'éléments siliceux amorphes plus ou moins ténus; à la Mougudo, à Saint-Vincent, la végétation à caractère tropical qui décorait le paysage pendant les périodes de repos des phénomènes volcaniques, était périodiquement ensevelie sous des amas de cendres ou cinérites qui en assuraient la conservation, avec tous les détails merveilleux des tissus les plus délicats.

La monographie de nos dépòts diatomifères serait très instructive, et donnerait lieu à des aperçus tout à fait inattendus. Il serait curieux, par exemple, de rechercher dans quelles conditions se sont formés les dépóts remaniés de Varenne, d'Auxillac et de Neussargues. Dans ces dépòts, on trouve des Coscinodiscus bien définis; or, aujourd'hui, 
tous les Coscinodiscus sont marins; il n'est done pas possible que ces dépôts se soient formés dans des lacs d'eau. douce; les Coscinodiscus n'ont pu vivre et se développer que dans des lacs profonds, alimentés par de puissantes sources thermo-minérales et riches en éléments salins.

Mais que savons-nous des eaux, considérées aujourd'hui comme douces, de l'époque tertiaire, et surtout en Auvergne? - Fiat lux!!...

\section{DE L'INFLUENCE DE LA LUMIÈRE ET DE L'ALTITUDE}

SUR LA STRIATION DES VALVES DES DIATOMÉES

$1^{\circ}$ Influence de la lumière. - "Le degré d'éclairement ne peut-il pas modifier sensiblement la striation des valves siliceuses des Diatomées? Par exemple, la striation d'une espèce vivant sur les bords ensoleillés d'un lac est-elle identique á celle de la même espèce se développant á une profondeur considérable, oì la lumière n'arrive que trìs affaiblie (1) ? "

A l'époque oì nous nous posions ces deux questions (octobre 1891), nous ne possédions pas encore les éléments d'étude nécessaires pour les résoudre d'une façon satisfaisante. Aujourd'hui, grâce aux sondages effectués dans plusieurs lacs d'Auvergne, par MM. le professeur $\mathrm{D}^{\mathrm{r}} \mathrm{P}$. Girod, A. Berthoule, Ch. Bruyant, A. Eusébio et P. Gautier, il nous. a été possible d'élucider ce point spécial concernant le développement de nos petites Algues.

Des Characées du lac Chauvet (Chara fragilis et hispida, Nitella translucens et flexilis), provenant d'une profondeur de $15^{\mathrm{m}}$, nous ont fourni une série de Diatomées

(1) Les Dial. diduv., Intr., p. 13. 
vivantes dont il nous a été facile de comparer la striation avec celle des mémes espèces récoltées sur les bords du même lac. L'examen de ces deux catégories de Diatomées nous a permis de constater les faits suivants:

$1^{\circ}$ Les espèces vivant à la profondeur de $15^{\mathrm{m}}$ se montrent normalement endochromées, et les chromatophores sont mème plus vivement foncés que ceux des espèces développées au bord du lac, exposées à l'action directe des rayons solaires ;

2 La forme du frustule est généralement plus allongée et moins large;

$3^{\circ}$ Le nombre des stries diminue par l'affaiblissement de la lumière.

Voici, d'ailleurs, les espèces sur lesquelles nous avons observé ces faits:

Par $15 \mathrm{~m}$ sous l'eau. Bords du lac.

Gomphonema cupitatum Ehrb... 6 à 9 stries en $10 \mu .10$ à 14

Navicula elliptica Ktz........ 7 à $9 \quad$-. $\quad 10$ à 13

- radiosa Ktz........6 a 8 a 8 - 9 à 12

- cardinalis Ktz...... 5 à $7 \quad$ - 7 à 10

- mesolepta Ehrb......9 à $12 \quad$ - 13 à 18

Stauroneis Phonicenteron Ehrb.. 9 à $12 \quad$ - 14 à 16

Synedra acuta Ktz..........9 à $11 \quad$ - 12 à 16

- Ulna Ehrb.......... 7 à $9 \quad$ - 10 à 13

Deux autres séries de Diatomées observées sur les Isoetes lacustris et echinospora du lac Guéry, les uns cueillis aux

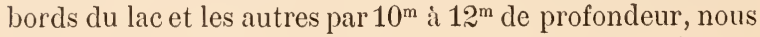
ont fourni un résultat analogue.

Bien que nos observations ne concernent jusqu'ici que le lac Chauvet et le lac Guéry, il est à croire que les autres lacs d'Auvergne permettraient de constater les mêmes faits, et nous croyons pouvoir conclure que l'influence de la lumière sur la striation des valves des Diatomées est un fait acquis à la science.

20 Influence de l'altitude. - L'influence de l'altitude sur la striation des valves des Diatomées a été déja soup- 
çonnée par Schulmann (1) et par le professeur J. Brun (2); mais ces deux diatomistes n'ont point précisé ce fait important.

M. J. Brun, le savant micrographe de la Faculté de Genève, nous écrivait, en effet, à la date du 15 décembre 1891 :

"Pour les variations que subit la striation de la valve, sous l'influence de l'altitude, il s'agirait de comparer, non les espèces de la mème masse d'eau, mais des exemplaires de la même espèce récoltée dans la plaine et sur les hautes montagnes. Cette donnée, mal élucidée par les diatomistes, serait, je crois, fort intéressante. »)

Au cours de nos recherches sur les Diatomées d'Auvergne, nous avons constaté que l'altitude augmente le nombre des stries et diminue leur intensité; en d'autres termes, pour une même espèce cueillie dans la plaine et sur les sommets de nos plus hautes montagnes, les stries de la forme alpine sont plus nombreuses et moins fortes.

Les récoltes examinées, pour le département du Puyde-Dòme, avaient été prises près du sommet du pic de Sancy, á une altitude de $1830^{\mathrm{m}}$ environ, et dans un étang près de Lezoux, altitude de $350^{\mathrm{m}}$.

Les espèces du Cantal provenaient d'une source froide située près du sommet du Plomb, ì une altitude de $1800^{\mathrm{m}}$, et des bords du Lot, à Vieillevie, al titude $\underset{22}{20}$.

Voici les espèces étudiées comparativement :

Forme alpine. Forme de la plaine.

Gomphonema dichotomum Kitz... 14 à 17 stries en $10 \mu .11$ à 14

Cymbella Ehrenbergii Kitz..... 7 à $9 \quad 5$ à 7

Navicula cuspidata Ktz.......14 à $18 \quad$ - 11 à 13

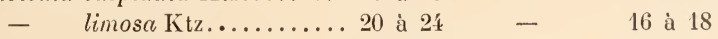

_ riridis Ktz......... 10 à $13 \quad$ - 7 à 9

Synedra capitata Ehrb........ 12 à $15 \quad$ - 9 à 11

(1) Diat. du IIant-Tatra, 1867, p. 38 .

(2) Diat. des Alpes et du Jura, 1880, p. 18. 
Tels sont les faits que nous avons constatés et que nous nous proposons de vérifier encore dans nos recherches ultérieures.

Conclusions. - $1^{\circ}$ Sous l'influence d'un éclairement affaibli, voisin probablement de l'obscurité physiologique, qui existe á une profondeur de $15^{\mathrm{m}}$ à $20^{\mathrm{m}}$ dans les lacs d'Auvergne, la striation des valves des Diatomées se montre moins serrée; de plus, la forme générale des frustules est plus allongée et plus étroite.

2. Sous l'influence de l'altitude, les stries sont plus nombreuses et moins fortes.

Nous donnons, dans les tableaux suivants, avec la liste des Diatomées fossiles qui n'ont pas été trouvées à l'état vivant, du moins en Auvergne, les florules des principaux dépôts des deux départements.

Les diatomistes ne manqueront pas de constater l'intérèt tout spécial que présente cette partie de la flore diatomique de la province; c'est, en effet, dans les dépóts fossiles, toujours féconds en surprises, que nous avons trouvé presque toutes nos espèces et variétés nouvelles. Malgré l'importance des résultats acquis, nous n'avons pas la prétention d'avoir tout vu; le beau dépót de Celles, en particulier, n'est pas encore suffisamment connu ; nous n'avons pu lui consacrer qu'un petit nombre de semaines d'examen, alors qu'il exigerait plusieurs années de recherches.

Nous le recommandons á ceux de nos confrères en diatomologie qui s'intéressent, de préférence, à l'étude des Diatomées fossiles. 


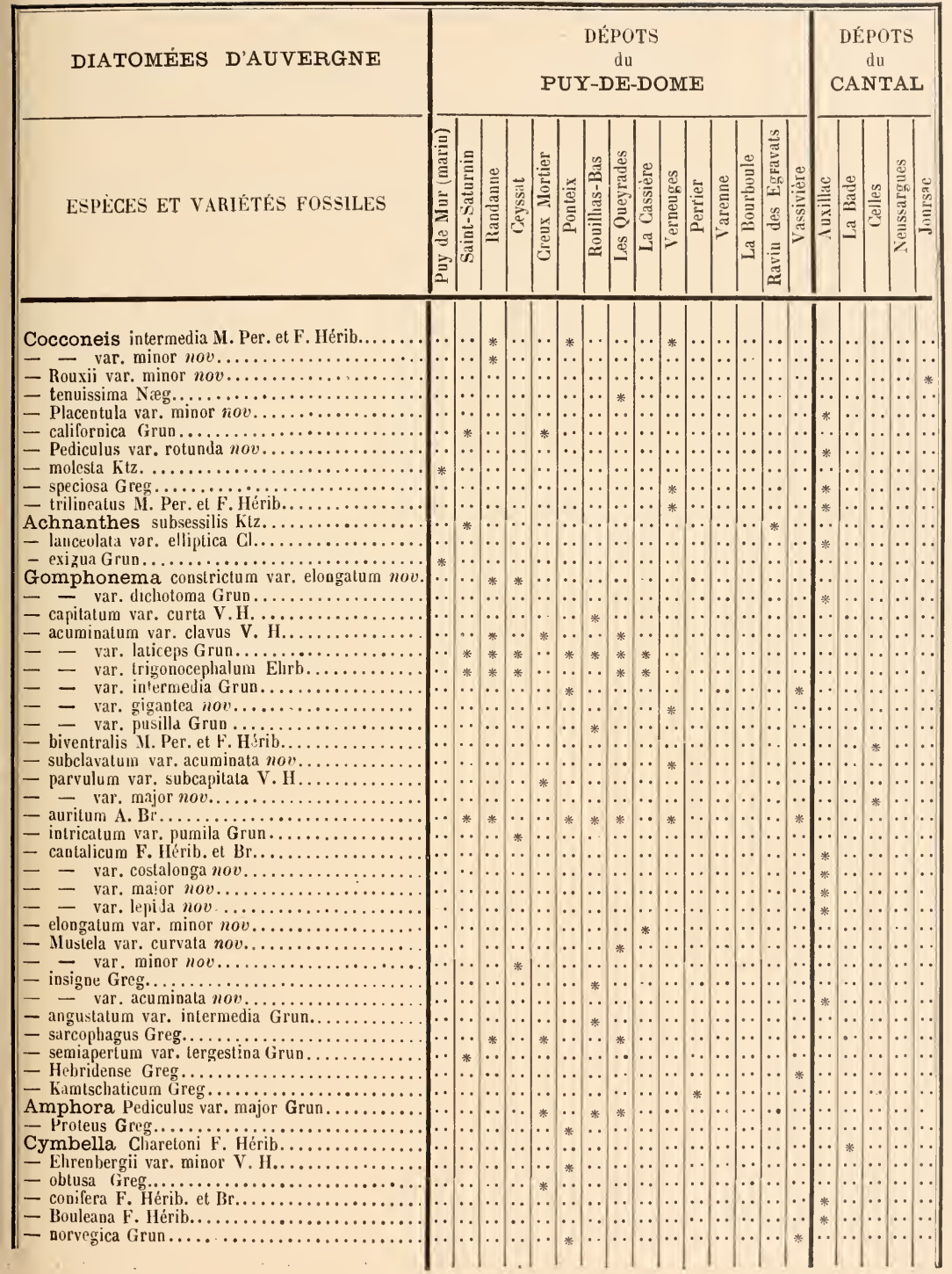




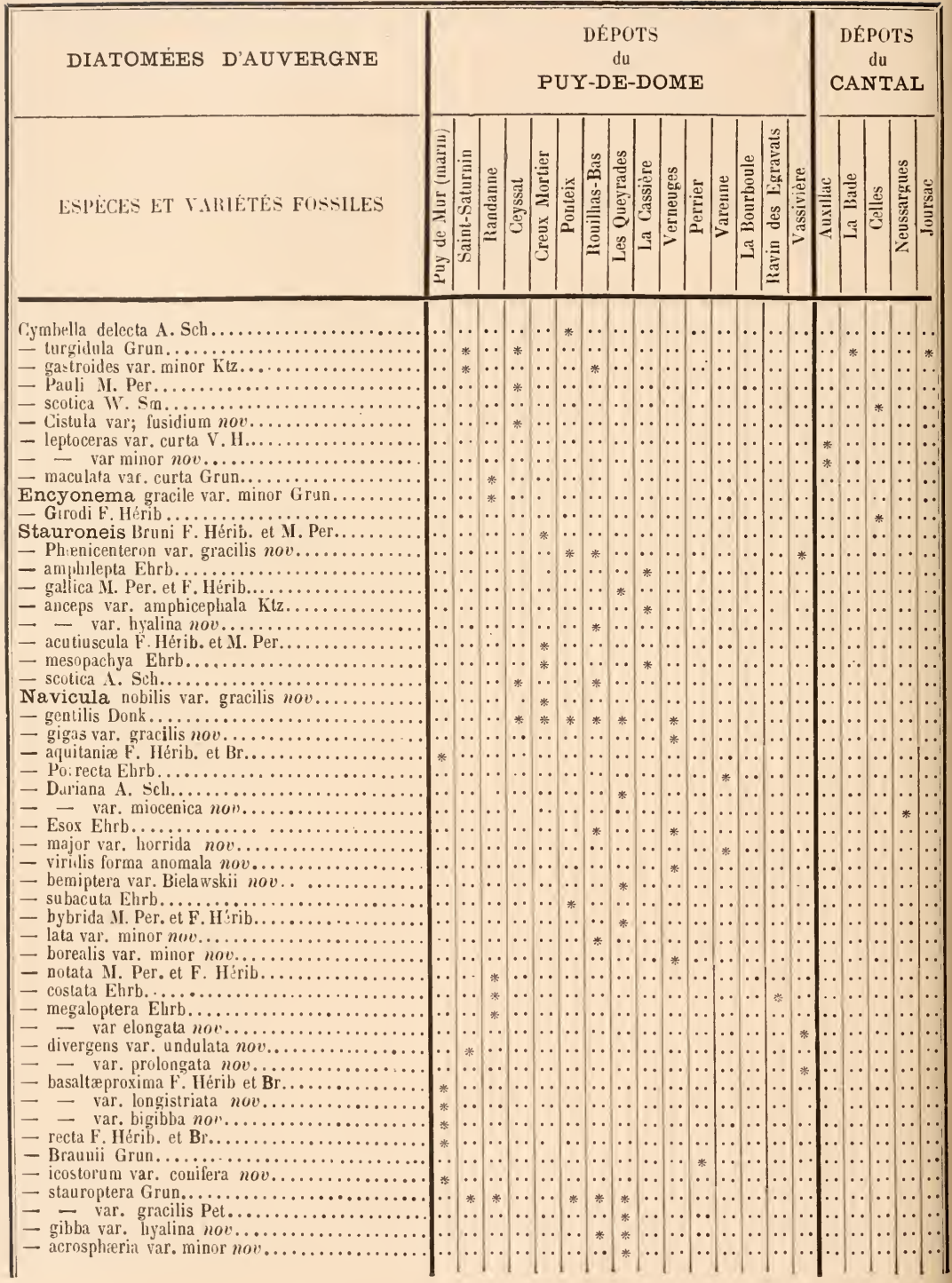


FLORULES DE NOS PRINCIPAUX DÉPÓTS.

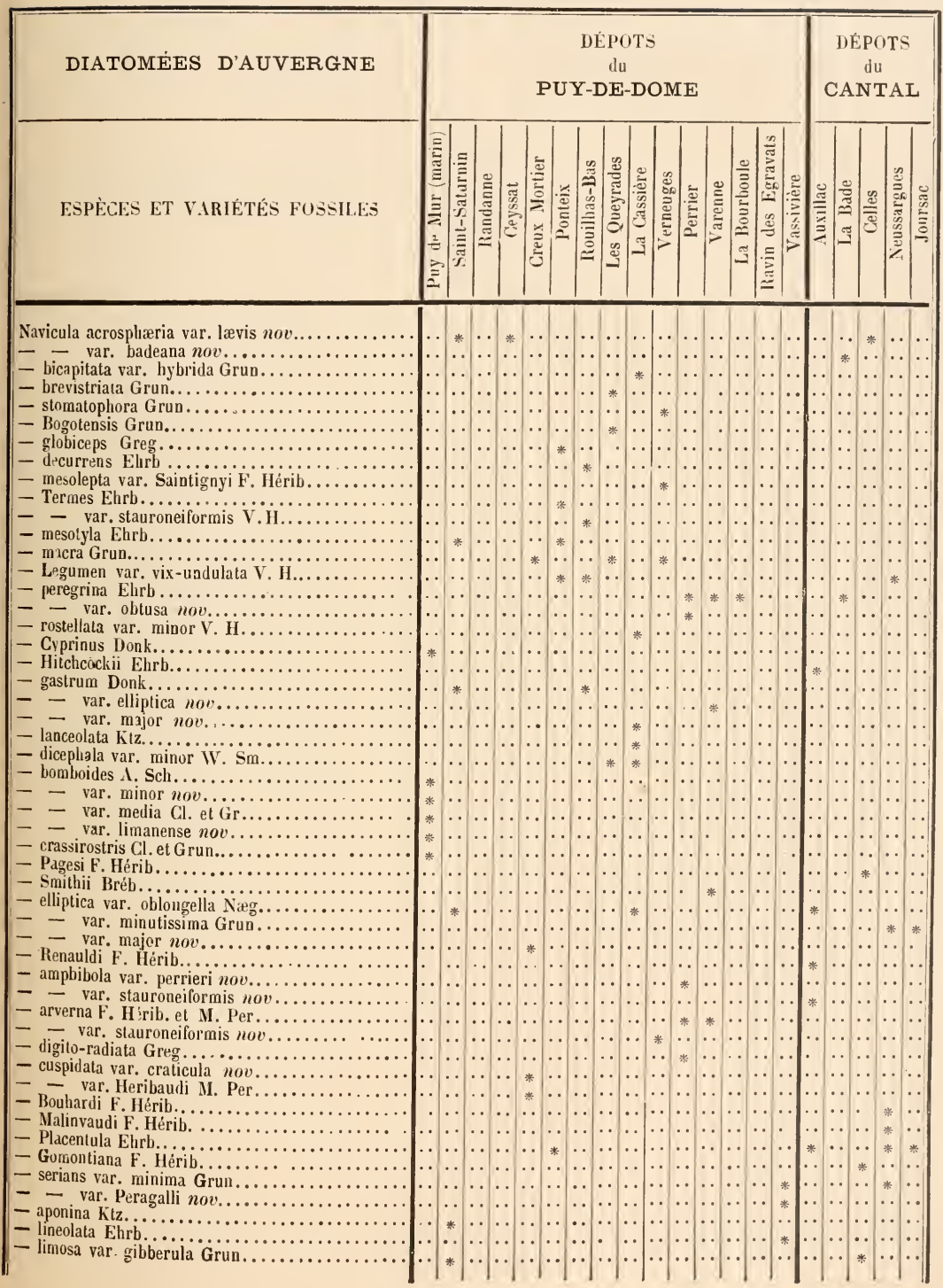


LES DIATOMÉES FOSSILES D'AUVERGNE.

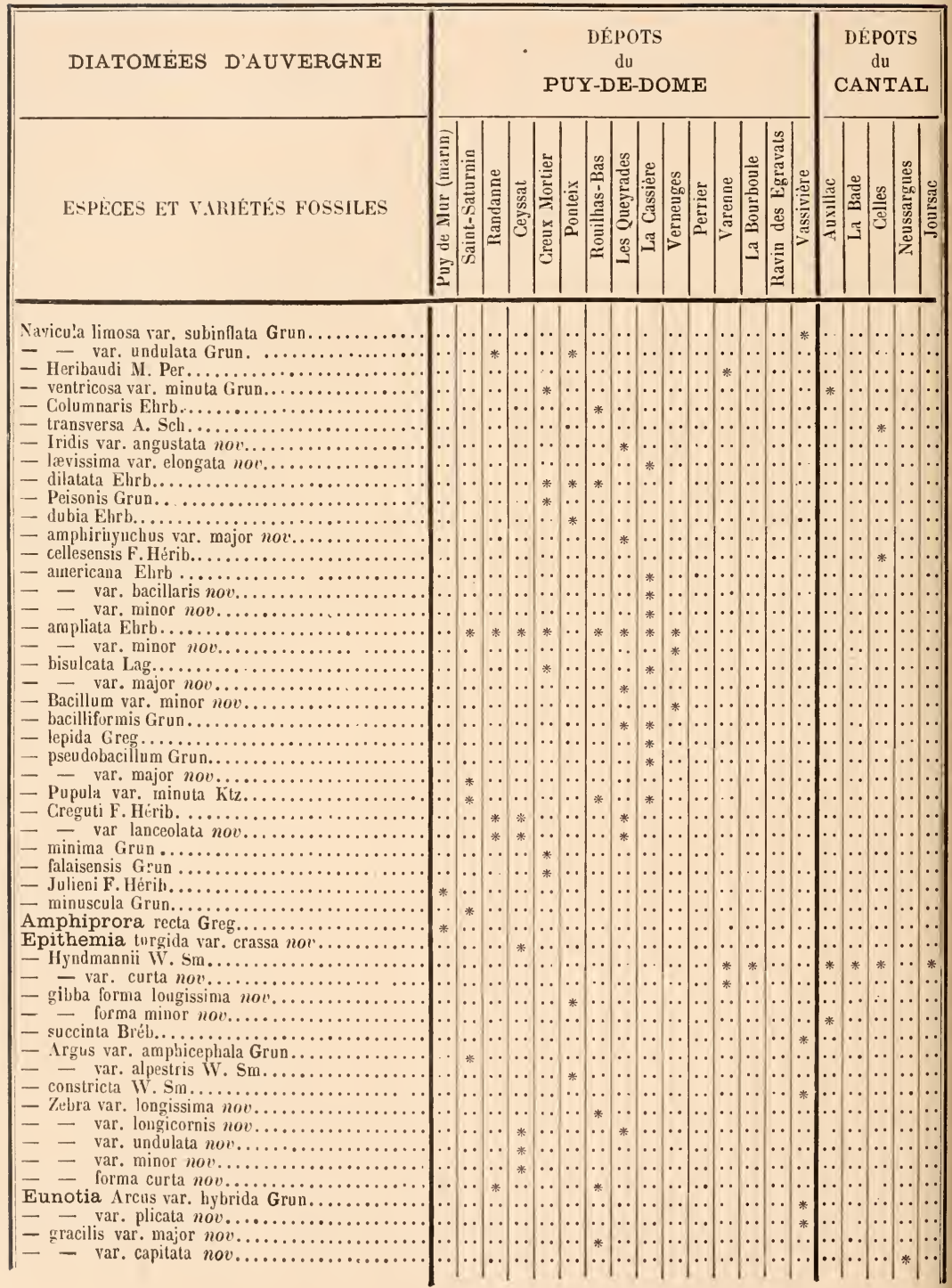




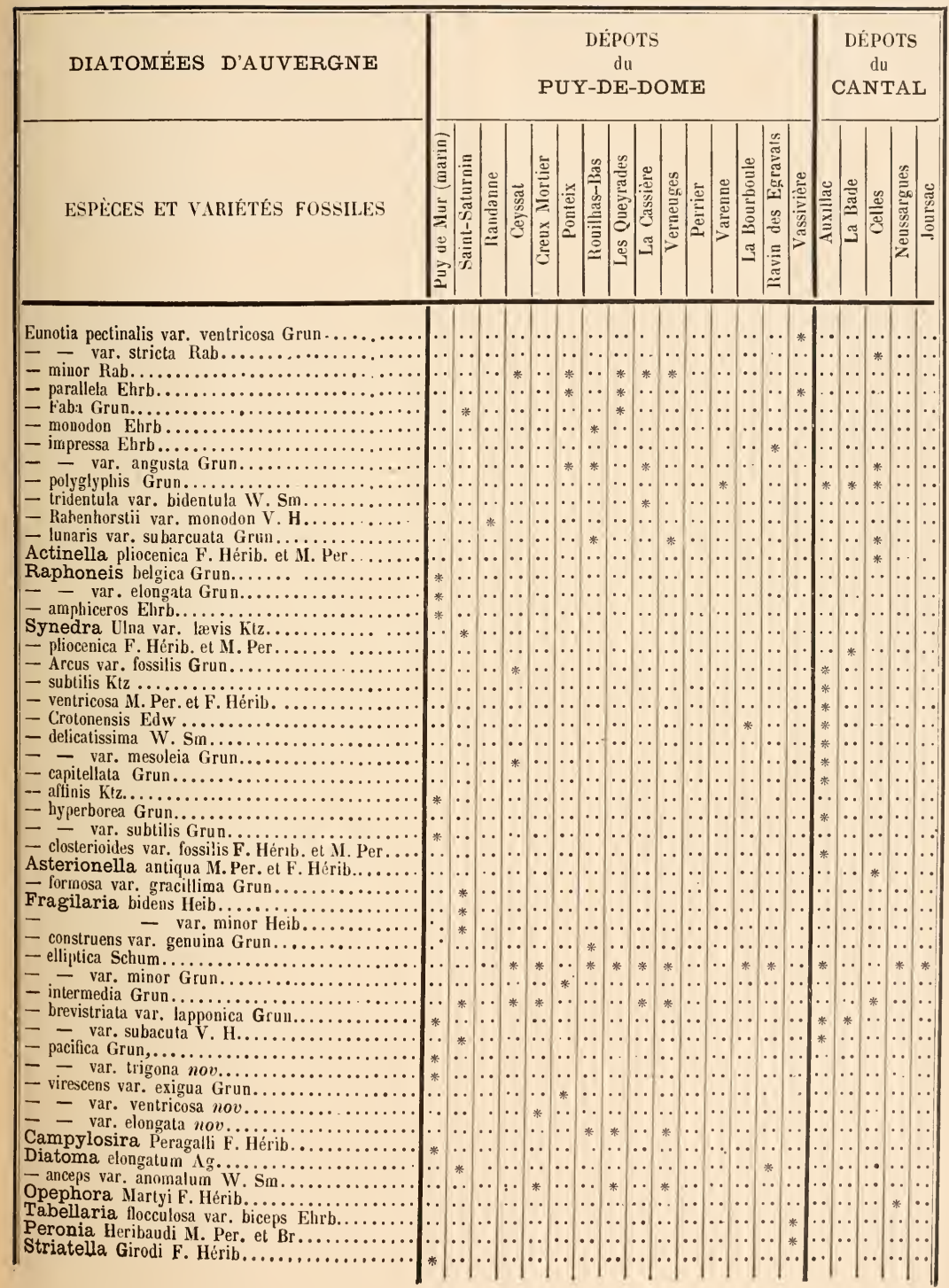




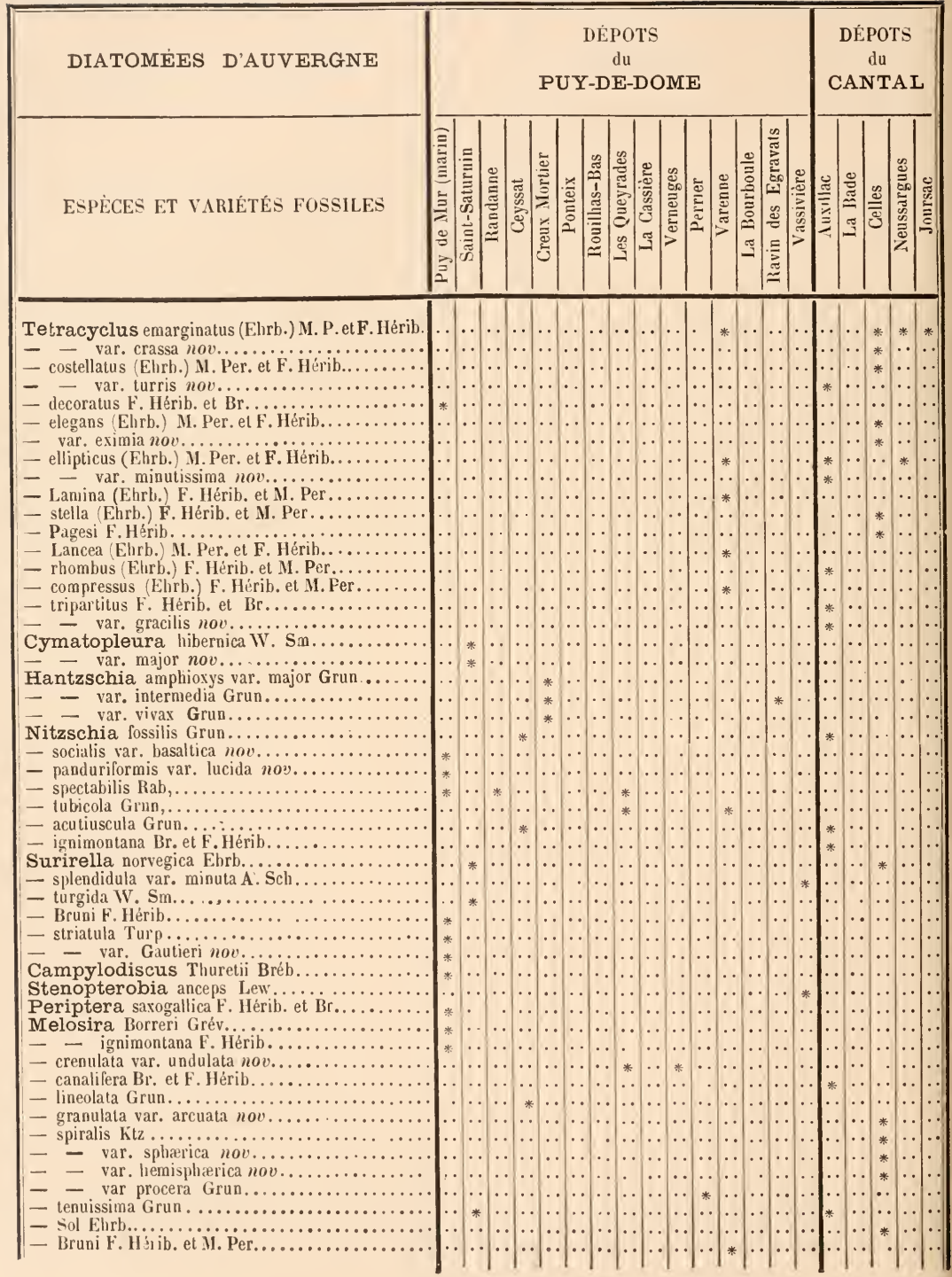




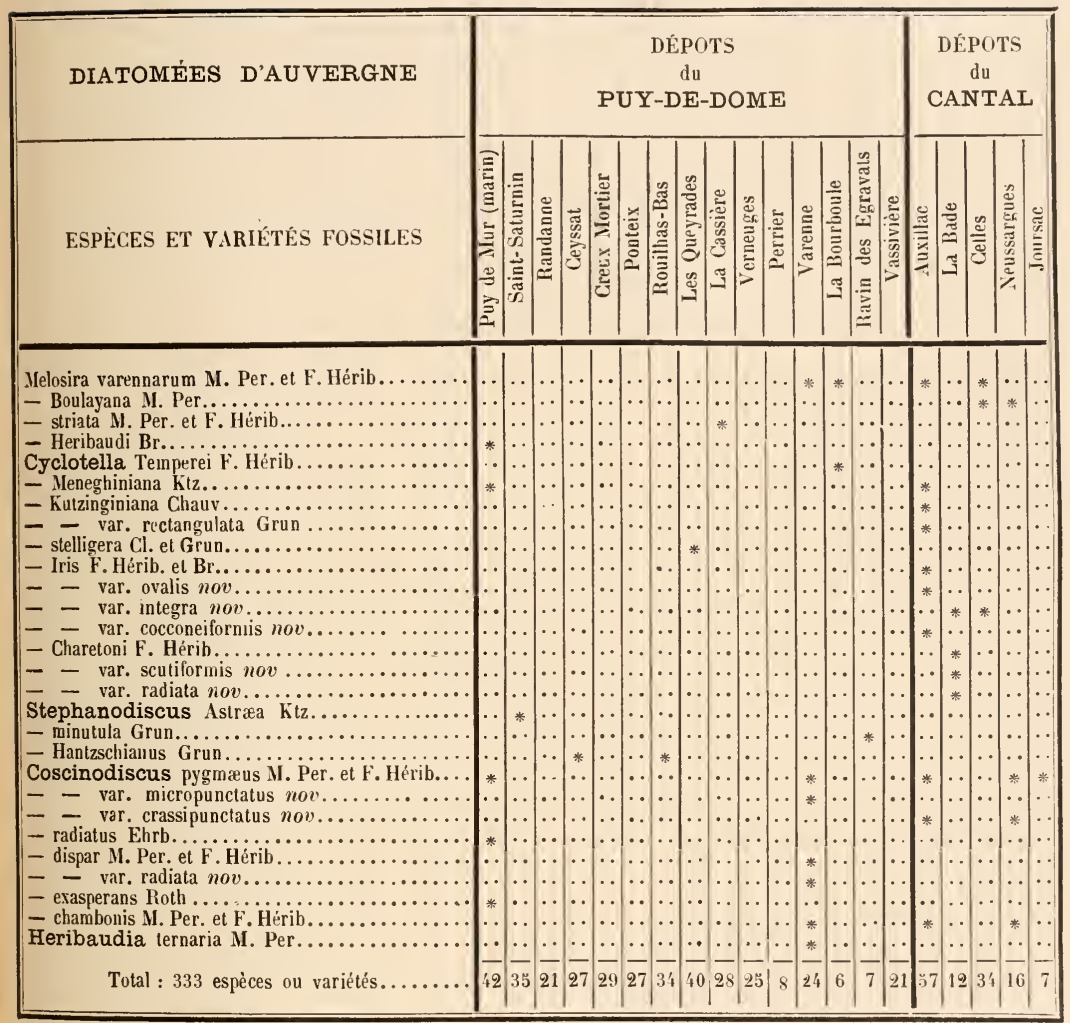

Parmi ces 333 espèces ou variétés fossiles, il en est au moins une centaine, appartenant aux dépòts quaternaires, qui ont été trouvées à l'état vivant, soit en France, soit dans d'autres régions de l'Europe centrale; des recherches ultérieures permettront très probablement de constater que la plupart de ces Diatomées vivent aussi en Auvergne.

Quelques-uns de nos dépôts sont extrêmement intéressants pour les diatomistes qui s'occupent de préférence 
des espèces fossiles; tels sont : le très curieux dépót marin du Puy de Mur, avec ses nombreuses espèces franchement marines; les dépòts tertiaires de Saint-Saturnin, de Varenne, d'Auxillac, de la Bade et de Celles; les dépóts quaternaires de Ceyssat, du Creux Mortier, de Ponteix, des Queyrades, de Verneuges et de Vassivière.

Nous pouvons procurer tous les dépôts d'Auvergne, en échantillons très authentiqucs, aux diatomistes qui désireraient les posséder à titre d'éléments d'étude. 


\section{PLANCHE VII}




\section{PLANCHE VII.}

Fifi.

1. Naricula Bouhardi F. Hérib.

2. - acrospharia var. badeana M. Per. et F. Hérib.

3. - basaltaproxima var. longistriata F. Hérib. et M. Per.

4. - viridis forma anomala M. Per. et F. Hérib.

5. - mesolepta var. Saintignyi F. Hérib.

6. - Malinoaudi F. Hérib.

7. -- Pagesi F. Hérib.

8. - Dariana var. miocenica M. Per. et F. Hérib.

9. - Renauldi F. Hérib.

10. - arverna var. stauronciformis M. Per. et F. Hérib.

11. - amphibola var. perrieri M. Per. et F. Hérib.

12. - - var. stauroneiformis F. Hérib. et M. Per.

13. - cellesensis F. Hérib.

14. -- Gomontiana F. Hérib.

15. - lomboides var. limanense F. Hérib. et M. Per.

16. - - var. minor F. Hérib.

17. Cymbella Charetoni F. Hérib.

18. Encyonema Girodi F. Hérib.

19. Synedra pliocenica F. Hérib. et M. Per. 
Dialomées d'Auvergne

PI. VII
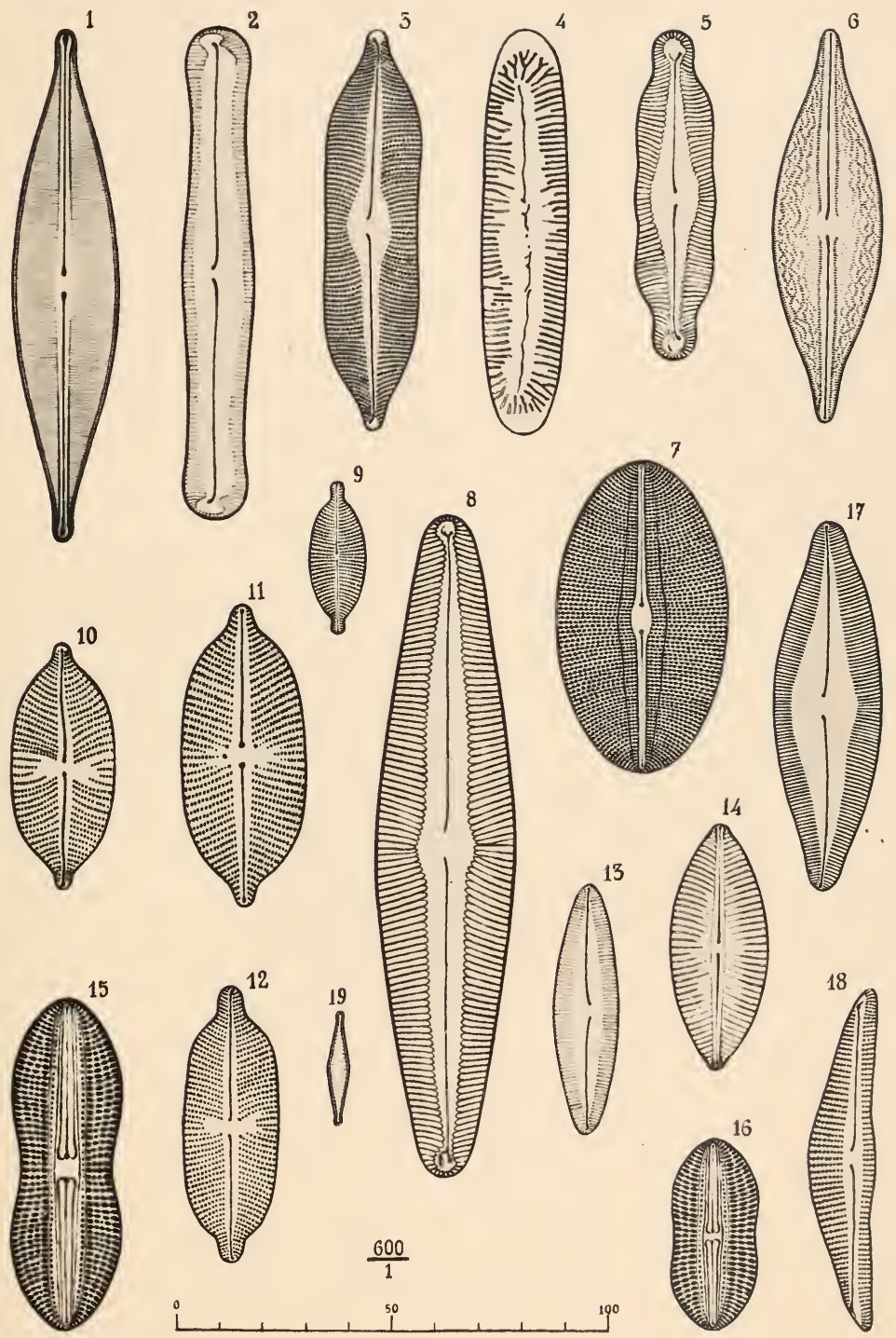

M.Peragallo del. 
$-$ 
PLANCHE VIII 


\section{PLANCHE VIII.}

Fig.

1. Gomphonema cantalicum var. lepida F. Hérib. et M. Per.

2. - acuminatum var. gigantea M.Per.et F. Hérib.

3. - biventralis M. Per. et F. Hérib.

4. - - insigne var. acuminata F. Hérib. et M. Per.

5 et 6 . Synedra closterioides var. fossilis M. Per. et F. Hérib.

5 , deux frustules accolés vus par leur face connective;

6 , face valvaire.

7. Actinella pliocenica F. Hérib et M. Per.

s. Asterionella antiqua M. Per. et F. Hérib.

9. Tetracyclus stella (Ehrb.) M. Per. et F. Hérib.

10. - Pagesi F. Hérib.

11. - tripartitus var. gracilis F. Hérib. et M. Per.

12. - costellatus (Ehrb.) M. Per. et F. Hérib.

13. - - var. turris F. Ilérib. et M. Per. (anneau connectif).

14. - elegans (Ehrb.) M. Per. et F. Hèrib.

15. -- - var. eximia F. Hérib. et M. Per.

16. - emarginatus var. crassa M. Per. et F. Hérib.

17, 18 et 19. Campylosira Peragalli F. Hérib.

17 , face valvaire; 18 , face connective; 19 , déduplication.

20. Opephora Martyi F. Hérib.

21. Eunotia gracilis var. capitata M. Per. et F. Hérib.

22 et 23. Cyclotella Temperei F. Hérib.

22 , face connective; 23 , face valvaire.

2't et 26. Melosira spiralis var. hemisphcerica M. Per. et F. Hérib.

24 , deux frustules accolés; 26 , face valvaire.

2.5. - - var. spharica F. Hérib. et M. Per.

27 et 28. - Boulayana M. Per.

27 , valve supérieure; $\mathbf{2 8}$, valve inférieure.

29. Cyclotella Iris F. Hérib. et Br. (forma typica).

30. - Charetoni F. Hérib.

31. - - var. integra F. Hérib. et M. Per. 
Dialomees d'Auvergne

PI. VIII
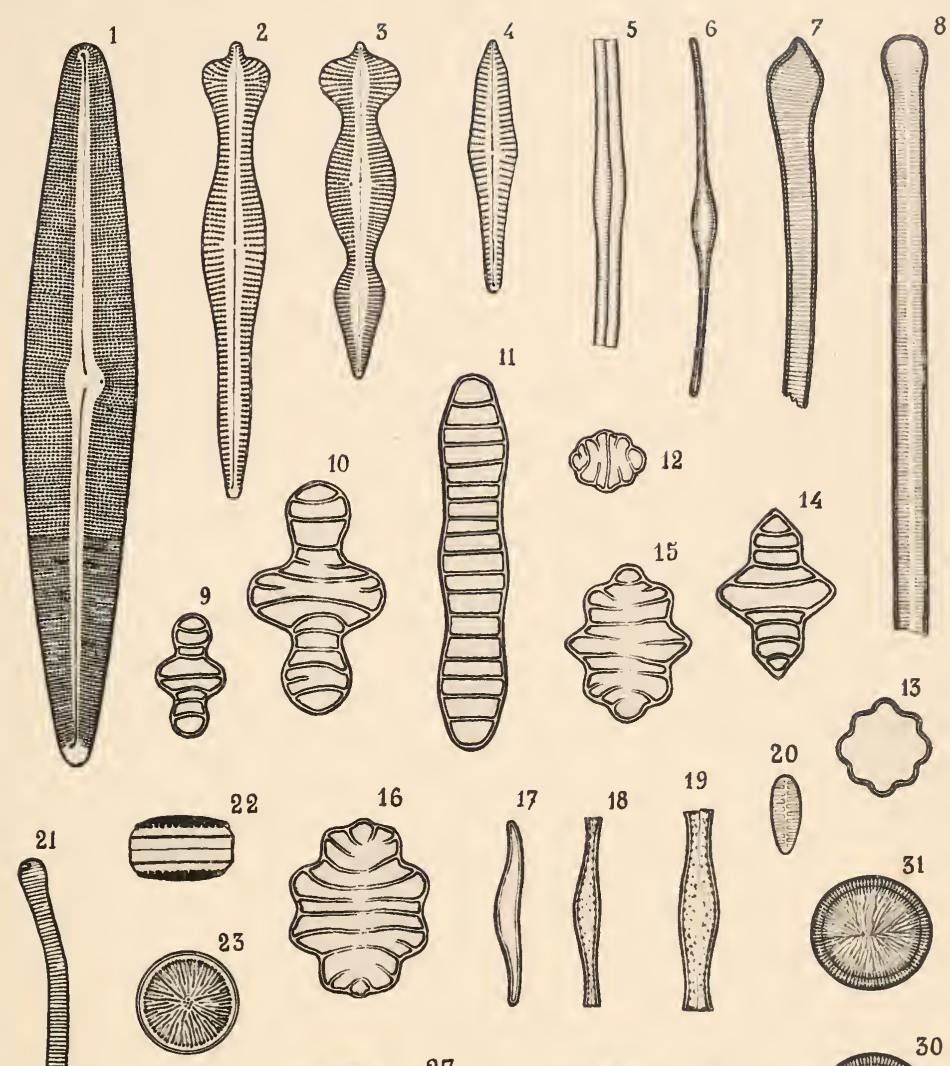

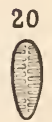
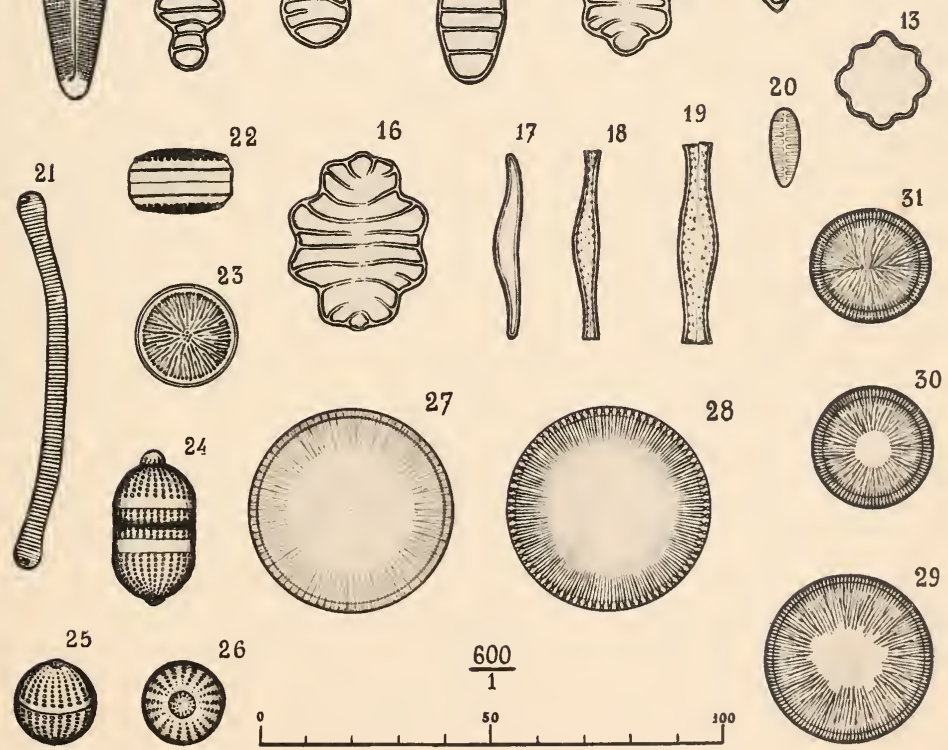

M.Perogallo del. 



\section{CATALOGUE ALPHABÉTIQUE}

DES

\section{DIATOMÉES D'AUTERGNE}

Achnanthes Biasolettiana Grun.

- coarctata Grun.

- delicatula Grun.

- exigua Grun.

— exilis Kiz.

- flexella Bréb.

- - var. alpestris $\mathrm{J} . \mathrm{Br}$.

- gibberula $\mathrm{Cl}$.

- hungarica Grun.

- lanceolata Grun,

- var. elliptica $\mathrm{Cl}$.

- microcephala Grun.

- minutissima Kiz.

- Peragalli F. Hérib. et $\mathrm{Br}$.

- subsessilis Ktz.

- trinodis Grun.

Actinella pliocenica F. Hérib. Amphipleura pellucida $\mathrm{K} t \mathrm{z}$. Amphiprora recta Greg. Amphora acutiuscula Ktz.

- affinis Klz.

- Ergadensis Greg.

- gracilis Ehrb.

- hyalina Kiz.

- Normanii Rab.

- ovalis Ktz.

- Pediculus Grun.

- - var. exilis Grun.

- $\quad$ - var. major Gruı.

- - var. minor Gruil.
Amp. Proteus Greg. — salina W. Sm.

- veneta Kiz.

Asterionella antiqua F. Hérib.

- formosa Hass.

- - var. gracillima Grun.

Campylodiscus costatus $\mathrm{W}$. Sm.

- noricus Ehrb.

- Tlıuretii Bréb.

Campylosira Peragalli F. Ilirib.

Ceratoneis Ircus Kit.

- var. ampbioxys Rab.

Cocconeis californica Grun.

- intermedia F. Hérib. et $\mathbf{3 1}$. Per.

- - var. minor nou".

- lineata Grun.

- - var. euglypta Grun.

- - forma minor noe.

- molesta Ktz.

- Pediculus Ehrb.

- - var. rotunda nov.

- Placentula Elirb.

- - forma minor noc.

- Rouxii F. Hérib.

- - var. minor nov.

- salina Rab.

- speciosa Greg.

- tenuissima Nieg.

- trilineatus F. Hérib, et M. Per.

Coscinodiscus chambonis nol. 
Cosc. exasperans Roth.

- dispar F. Hérib. et Br.

- var. radiata nov.

- pygnæus M. Per. et F. Hérib.

- - var. micropunctata nov.

- - var. crassipunctata nov.

- radiatus Ehrb.

Cyclotella bodanica Eul.

- Charetoni F. Hérib.

- - var scutiformis nov.

- - var. radiata nov.

- comensis Grun.

- comla Ktz.

- var. arverna nov.

- Iris F. Hérib. et $\mathrm{Br}$.

- - var. integra nov.

- _ var. ovalis nov.

- - var. cocconeiformis nov.

- Kutzingiana Chauv.

- Meneghiniana $\mathrm{K} \mathrm{z}$.

- - var. rectangulata Grun.

- operculata Kiz.

- - var. antiqua Wr. Sm.

- stelligera Cl. et Grun.

- Temperei F. Hérib.

Cymatopleura elliptica WV. Sm.

- - var. constricta Grun.

- - var. subconstricta Grun.

- hibernica W. Sm.

- - var. major nov.

- Solea Bréb.

- - var. apiculata Pritch.

Cymbella affinis Ktz.

- alpina Grun.

- - forma minor nov.

- amphicephala Næg.

— anglica Lag.

- aspera Elirb.

- Bouleana F. Hérib.

- Charetoni F. Hérib.

- Cistula Hempr.

- - var. fusidium nov.

- conifera F. Hérib. et Br.

- cuspidata Ktz.

- cymbiformis Elirb.

- delecta A. Sch.

- Ehrenbergii Greg.

- -- var. minor V. H.

- gastroides kitz.

- - var. minor V. H.
Cymb. helvetica Ktz.

- lævis Næg.

- lanceolata Ehrb.

- leptoceras Ktz.

- - forma curta nov.

- - forma minor nov.

- maculata Kı.

- - forma curta Grun.

- microcephala Grun.

- naviculiformis Auersw.

- norvegica Grun.

- obtusa Greg.

- parva W. Sm.

- Pauli M. Per.

- pusilla Grun.

- stomatophora Grun.

- subæqualis Grus.

- tumida Bréb.

- turgidula Grun.

Denticula elegans Kitz.

- - var. thermalis Kiz.

- frigida Kitz.

- inflata W. Sm.

- tenuis Ktz.

- - var. intermedia Grill.

- - var. mesolepta Grun.

Diatoma anceps Grun.

- - var. anomalum W. Sm.

- Ehrenbergii Ktz.

- - var. Grande W. Sm.

- elongatum $\mathrm{Ag}$.

- hyemale Heib.

- Mesodon Ktz.

- pectinale Ktz.

- teuue Ag.

- vulgare Bory.

- - var, lineare W. Sm.

Encyonema cæspitosum $\mathrm{K} t z$.

- - var. lata V. H.

- Girodi F. Hérib.

- gracile Rab.

- - var. lunata W. Su.

- - forma minor Grun.

- lunata Grun.

- Pediculus Kiz.

- prostratum Ralfs.

- turgidum Grun.

- ventricosum $\mathrm{K} / \mathrm{z}$.

- - var. excisa nov.

- _ var, minuta Hilse. 
Epithemia Argus Ktz.

- - var. amphicephala Grun.

- - var. alpestris W. Sm.

- constricta W. Sm.

- gibba Ehrb.

- - var. parallela Grun.

- - var. ventricosa Grun.

- - forma longissima nov.

- gibberula Ehrb.

- - var. producta Grun.

- Hyndmannii W. Sm.

- - var. curta nov.

- ocellata Ehrb.

- rupestris W. Sin.

- Sorex Kitz.

- succincta Bréb.

- turgida Ktz.

- - var. granulata Grun.

- - var. Vertagus Kiz.

- - forma crassa nov.

- Westermannii Ktz.

- Zebra Ktz.

- - var. minor nov.

- - var. longicornis nov.

- - var. longissima nov.

- - var. proboscidea Grun.

- $\quad$ var. undulata nov.

- - forma curta nov.

Eunotia Arcus Ebrb.

- - var. bidens Grun.

- - var. hybrida Grun.

- - var. plicata nov.

- Faba Grun.

- flexuosa Kíz.

- - var. bicapitata Grun.

- gracilis Rab.

- - var. capitata nov.

- - var. major nov.

- impressa Ehrb.

- - var. angusta Grun.

- incisa Greg.

- lunaris Grun.

- - var. bilunaris Grun.

- - var. excisa Grun.

- - var. subarcuata Grun.

- major Rab.

- - var. bidens W. $s m$.

- minor Rab.

- monodon Ehrb.

- - var. diodon Ehrb.
Eun. - var. hendecaodon Ralfs.

- paludosa Grun.

- parallela Ehrb.

- pectinalis Rab.

- - var. elongata Rab.

- - var. stricta Rab.

- - var. undulata Ralfs.

- - var. ventricosa Grun.

- polyglyphis Grun.

- prærupla Ehrb.

- - var. inflata Grun.

- - var. bigibba Ktz.

- Rabenhorstii Cl. et Grun.

- robusta Ralfs var. tetraodon Ehrh.

- tridentula Ehrb.

- var. bidentula W. Sm.

Fragilaria æqualis Lag.

- bidens Heib. forma major Heib.

- binodis Ebrb.

- - var.obliqua nov.

- brevistriata Grun.

- - var. lapponica Grun.

- - var. Mormorum Grun.

-- - var. pusilla Grun.

- - var. subcapitata Grun.

- capucina Desm.

- - var. acuminata Grun.

- - var. acuta Grun.

- - var. mesolepta Grun.

- construens Grun.

- - var. capitata nov.

- - var. genuina Grun.

- - var. pumila Grun.

- - var. Venter Grun.

- elliptica Schum.

- $\quad$ - forma minor Grun.

- Harrisonii Grun.

- hyalina Grun.

- intermedia Grun.

- lapponica Grun.

- mutabilis Grun.

- nitzschioides Grun.

- - var. brasiliensis Grun.

- pacifica Grun.

- - var trigona nov.

- parasitica Grun.

- - var. subconstricta Grun.

- producta Grun.

- striatula Lyngb.

- undata $\mathrm{W}$. Sm, 
Fr. virescens Ralfs.

- - var. elongata nov.

- - var. exigua nov.

- - var. ventricosa nov.

Gomphonema abbreviatum Kiz. - acuminatum Ehrb.

- - var. clavus V. H.

- - var. coronata Elirb.

- _ var. gigantea nov.

- - var, intermedia Grun.

- - var. laticeps Grun.

- - var. pusilla Grun.

- - var. trigonocephalum Ehrb.

- alfine Kitz.

- engustatum Grun.

- - var. intermedia Grun.

- - var. producta Grun.

- - var. subæqualis Grun.

- Augur Ehrb.

- - var. Gautieri V. H.

- auritum A. Br.

- biventralis F. Hérib. et M. Per.

- Brebissonii Ktz.

- cantalicum F. Hérib.

- - var. costalonga nov.

- - var. lepida nov.

- - forma major nov.

- capitatum Ehrb.

- - var. curta nov.

- clavatum Elirb.

- commutatum Grun.

- constrictum Elırb.

- - var. elongata nov.

- - var. subcapitata Grun.

- Cygnus Elirb.

- dichotomum W. Sm.

- elongatum WV. Sm.

- $\quad$ - var. minor nov.

- exiguum Ktz.

- geminatum $\mathbf{A g}$.

- Hebridense Greg.

- insigne Greg.

- - var. acuminata nov.

- intricatum Ktz.

- - var. dichotoma Grun,

- var. pumila Gruı.

- Kamischaticum Grun.

- micropus Kitz.

- - var. minor Grun.

- montanum Scliuns.
Gomph. - var. pumila Grun - Mustela Ehrb.

- - var. curvata nov.

- - forma minor nov.

-_ olivaceım Elırb.

- parvulum Ktz.

- - var. lanceolata Ehrb.

- - var. subcapitata V.H.

- Sarcophagus Greg.

- semiapertum var. tergestina Grun.

- subtile Ehrb.

- subclavatum Grun.

- - var. acuminata nov.

- - var. major nov.

- tenellum Kítz.

- Vibrio Ehrb.

Hantzschia amphioxys Grun.

- - var. intermedia Grun.

- - var. major Grun.

- - var. vivax Grun.

- elongata Grun.

Heribaudia ternaria M. Perag.

Mastogloia Dansei Thw.

- Smithii Thw.

Melosira arenaria Moor.

- Borreri Grev.

- - var. ignimontana nov.

- Boulayana M. Per.

- Bruni F. Hérib.

- canalifera F. Hérib. et Br.

- crenulata Kiz.

- - var. anbigua Grun.

- - var. undulata $n o v$.

- - var. valida Grun.

- Dickiei Klz.

- distans Ehrb.

- - var. alpigena Grun.

- granulata Ehrb.

- - var. arcuata nov.

- Heribandi J. Br.

- lævis Grun.

- lineolata Grun.

- lirata Ehrb.

- - var. lacustris Grun.

- nivalis W. Sm.

- orichalced Mertens.

- Rreseana Moor.

- Sol (Elırb.) Ktz.

- spiralis Ktz.

- - var. liemisphærica nor. 
Mel. - var. spliarica nov.

- striata F. Hérib. et M. Per.

- tenuis Grun.

- tenuissima Grun.

- undulata Kiz.

- - var. producta A. Sch.

- varennaruın M. Per. et F. Hérib.

- varians $\Lambda \mathrm{g}$.

Meridion circulare Ag.

- constrictum Ralfs.

Navicula acrosphæria Bréb.

- - var. badeana nov.

- _ var. lævis noc.

- - var. minor nov.

- acuminata W. Sm.

- aftinis Elorh.

- var. undulata Grun.

-- ambigua Ehrb.

- americana Elırb.

- - var. bacillaris nov.

- - forma minor nov.

- amphibola var. perrieri nov.

- - var. stau roneiformis nov.

- amphigomphus Ehrb.

- amphirhyncbus Ehrh.

- - var. major nov.

- amphisbæna. Bory.

- ampliata Elurb.

- - var. minor. nov.

- anglica Ralfs.

- aponina Kitz.

-- appendiculata Ktz.

- - var. irrorata Grun.

- aquitaniæ F. Hérib. et Br.

- - var. undulata nov.

- arverna F. Hérib. et M. Per.

- - var. stauroneiformis nov.

- atomoides Grun.

- atomus Grun.

- bacillaris Greg.

- bacilliformis Grun.

- Bacillum Elırb.

- basaltæproxiıa F. Hérib. et $\mathrm{Br}^{2}$.

- - var. bigibba uov.

— . var. longistriata nov. .

- bicapitata Lag.

- - var. lybrida Grun.

- biceps Greg.

- binodis W. Sm.

— bisulcata Lag.
Nav. - var. major noe.

- Bogotensis Grun.

- bomboides A. Sch.

- - var. limanense nuv.

- - var. media Grun.

- - var. minor nov.

- borealis Ktz.

- - var. major nov.

- - var. minor nov.

- Bouhardi F. Hérib.

- Braunii Grun.

- Brebisspnii Ktz.

- - var. diminuta Grun.

- - var. elongata nov.

- - var. ovalis H. Perag.

- - var. subproducta Grun.

- brevistriata Grun.

- cardinalis Ktz.

- cellesensis F. Hérib.

- Cesatii Rah.

- cincta Ktz.

- Columnaris Ehrb.

- costata Ebrb.

- crassinervia Bréb.

- crassirostris Cl. et Grun.

- Creguti F. Hérib.

- - var. lanceolata nov.

- rryptocephala Kiz.

- cuspidata kitz.

- $\quad$ var. Heribaudi nov.

- - forma craticula nov.

- Cyprinus W..Sm.

- Dactylus Ehrb.

- Dariana A. Sch.

- - var. miocenica $100 \mathrm{c}$.

- decurrens Elirh.

- digito-radiata Greg.

- dilatata Ehrb.

- dicephala W. Sm.

- - var. minor W. Sm.

- divergens W. Sm.

- - var. prolongata nov.

- - var. undulata noe.

- dubia Elırb.

- elliptica Ktz.

- - var. extenta W.Sm.

- - var. major nov.

- - var. minutissima Grun.

- - var. oblongella Næg.

- Esox Ehrb. 
Nav. exilis Grun.

- falaisensis Grun

- firma Ktz.

- gastrum Donk.

- - var. elliptica nov.

- - Coima major nov.

- gentilis Donk.

- gibba Ehrb.

- - var. hyalina nov.

- gigas Ehrb.

- globiceps Greg.

- Gomontiana F. Hérib..

- gracilis Ehrb.

- gracillima Pritch.

- Gregaria Donk.

- bemiptera Ktz.

- - var. Bielawskii not.

- Heribaudi M. Perag.

- Heufleri Grun.

- Hitchcockii Ehrb.

- humilis Donk.

- hybrida F. Hérib. et M. Per.

- icostauron var. conifera nov.

- Iridis Ehrb.

- - var. angustata nov.

- Julieni F. Hérib.

- lævissima Klz.

- - var. elongata nov.

- lanceolata Ktz.

- lata Bréb.

- - var. minor nov.

- Legumen Ehrb.

- - var. vix-undulata V. H.

- lepida Greg.

- leptocephala Bréb.

- limosa Kítz.

- - var. curta Grun.

- - var. gibberula Grun.

- - var. subinflata Grun.

- - var. undulata Grun.

- - forma major nov.

- lineolata Ehrb.

- longa Gireg.

- macra Grun.

- inajor Ktz.

- - var. horrida nov.

- - var. interrupta nov.

- Malinvaudi F. Hérib.

- megaloptera Ehrb.

- Nenisculus 1. Sch.

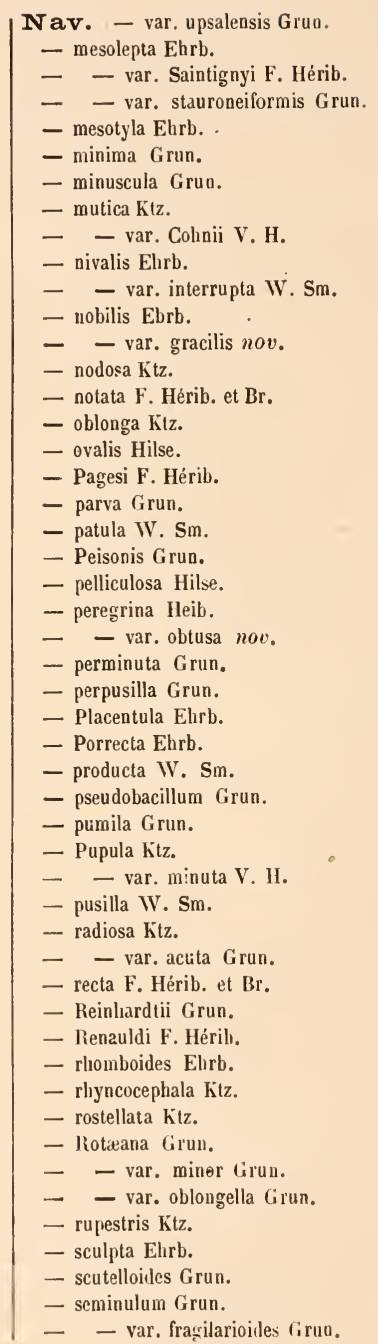


Nav. seriaus Ktz.

- - var. minima Girun.

- - var. minor Grun.

- - var. Peragalli nov.

- slesvicensis Grun.

- Smithii Bréb.

- sphærophora Ktz.

- stauroptera Grun.

- - var. gracilis P. Petit.

- stomatophora Grun.

- subacuta Elirb.

- subcapitata Greg.

- - var. paucistriata Grun.

- var. stauroneiformis Grun.

- Tabéllaria Elırb.

- tenella Bréb.

- Termes Ehrb.

- - var. stauroneiformis V. H.

- transversa A. Sch.

- trinodis W. Sin.

- tuscula Grun.

- ventricosa Donk.

- viridis Ktz.

- - var. curta A. Sch.

- - var. commutata Grun.

- - forma anomala nov.

— viridula kitz.

- vulgaris Heib.

- - var. lacustris. J. Br.

Nitzschia acicularis W. Sn.

- acutiuscula Grun.

- acuminata Grun.

- amphibia Grun.

- - var. Frauenfeldii Grun.

- angustata W. Sm.

— bilobata W. Sm.

- - var. liybrida Grun.

- Brebissonii IV. Sm.

- Calida Grun.

- communis Rab.

- - var. obtusa Grun.

- commutata Grun.

- constricta Greg.

- denticula Grus.

- dissipata Grun.

- - var. media Grun.

- dubia W. Sm.

- fonticola Grun.

- fossilis Grun.

- frustulum Grun.

Nitz. - var. Bulnlıeimiana frun.

- - var. minutula Rab.

- - var. perpusilla Rab.

- Hantzschiana Rab.

- hungarica Grun.

- ignimontana F. Hérib. et $\mathrm{Br}$.

- inconspicua Grun.

- Kittlii Grun.

- linearis WV. Sm.

- - var. major V. H.

- microcephala Grun.

- minuta Bleisch.

- obtusa WV. Sm.

- - var. scapelliformis firun.

- ovalis Arn.

- Palea Kiz.

- - var. exilis Grun.

- - var. tenuirostris Grun.

- panduriformis Greg.

- - var. lucida nov.

- recta $\mathrm{Htz}$

— sigmoidea Nitz.

- - var. armoricana Grun.

- sinuata Grun.

- socialis Greg.

- - var. basaltica nov.

- spectabilis Rab.

- subtilis Grun.

- Tabellaria Grun.

- tenuis Grun.

- thermalis Auersw.

- Tryblionella Htz.

- tubicola Grun.

- vermicularis Htz.

- Victoriæ Grun.

— vitrea Norm.

- - var. gallica nov.

Opephora Martyi F. Hérib.

Periptera saxogallica F. Hérib.

Peronia Heribaudi M. Per.

Pleurosigma acuminatum Grun.

- - var. scalproides Rab.

- attenuatum Ktz.

- Kutzingii Grun.

- Spencerii W. Sm.

Raphoneis amphiceros Elırb. - - forma minor Grun. - belgica Grun. - var. elongata Grun.

Rhoicosphenia curvata Gr. 
Rh. Van Heurchia Grun.

Rouxia Peragalli F. Hérib. et $\mathrm{Br}$. Stauroneis acuta W. Sm.

- acutiuscula F. Hérib. et M. Per.

- amphilepta Ebrb.

- anceps Ehrb.

- - var. amphicephala Ktz.

- - var. hyalina noe.

- Bruni F. Hérib.

- dilatata W. Sm.

- gallica F. Hérib. et M. Per.

- gracilis W. Sin.

- Legumen Ehrb.

- Mesopachya Ehrb.

- Phœnicenteron Elirb.

- - var. gracilis nov.

- - var. lanceolata J. Br.

- - forma crassa nov.

- platystoma Ehrb.

- scotica A. Sch.

- Smithii Grun.

Stenopterobia anceps Lewis. Stephanodiscus Astræa Kı.

- - var. minutula Grun.

- Hantzschianus Grun.

Striatella Girodi F. Hérib.

Surirella angusta Ktz.

- - var. contorta P. Petit.

- biseriata Bréb.

- - var. elliptica P. Petit.

- - var. linearis W. Sm.

- - var. subacuminata V. H.

- Bruni F. Hérib.

- Crumena Bréb.

- elegans Ehrb.

- gracilis Grun.

- - var. minor J. Br.

- helvetica J. Br.

- ovalis Bréb.

- norvegica Elırb.

- ovata Ktz.

- - var. minula Bréb.

- - var. pinnata W. Sm.

- patella Ehrb.

- robusta Ehrb.

- salina V. Sm.

- saxonica Auersw.

- spiralis Ktz.

- splendida Elirb.

- splendidula A. Scl.,
Sur. strialula Turp.

- - var. Gautieri F. Hérib.

- tenera Greg.

- - var. splendidula Greg.

- turgida W. Sm.

Synedra acuta Ktz.

- - var. oxyrhynchus Kitz.

- Acus Grun.

- - var. angustissima Grun.

- $\quad$ - var. fossilis Grun.

- - var. subtilis Grun.

- - var. ventricosa nov.

- delicatissima W. Sm,

- $\quad$ var. mesoleia Grun.

- affinis $\mathrm{Ktz}$.

- barbatula Kiz.

- capitata Ehrb.

- capitellata Grun.

- closterioides var. fossilis nor.

- Crotonensis Edw.

- delicatissima W. Sm.

- gracilıs Ktz.

- hyperborea Grun.

- pliocenica F. Hérib.

- radians Ktz.

- rumpens Grun.

- Ulna Ehrb.

- - var. amphirhynclus Ehrb.

- - var. bicurvata Grun.

- - var. danica Ktz.

- - var. lanceolata Kiz.

- - var. longissima W. Sm.

- - var. oblusa W. Sm.

- - var. spathulifera Grun.

- - var. subæqualis firun.

- - var. vitrea $\mathrm{Ktz}$.

- Vaucheriæ Ktz.

- - var. parvula kiz.

- - var. truncata $\mathrm{K} t \mathrm{z}$.

Tabellaria fenestrata Ktz.

- - var. nodosa Ehrb.

- - var. trinodis Ehrl.

- flocculosa Ktz.

- - var. biceps Ehrb.

Tetracyclus Braunii Grun.

- compressus (Ehrb.) nob.

- costellatus (Ehrb.) nob.

- - var. turris nov.

- decoratus F. Hérib. et $\mathrm{Br}$.

- emarginatus W. Sm. 
Tetr. - var. crassa nov.

- elegans (Ehrb.) nob.

- - var. eximia nov.

- ellipticus (Ehrb.) nob.

- - var. minutissima nov.

- Lamina (Ehrb.) nob.
Tetr. lancea (Ehrb.) nob.

- Pagesi F. Hérib.

- tripartitus F. Hérib. et Br.

- - var. gracilis nov.

- rhombus Ralfs.

- stella (Ehrb.) noh.

Les Diatomées énumérées dans notre Catalogue s'élèrent it 772 espèces ou variétés; tel est le résultat de nos patientes et longues recherches sur ces merveilleux microphytes de la Flore d'Auvergne.

Malgré l'importance relative des faits acquis, il reste encore beaucoup á découvrir; ainsi que nous l'avons dit plus haut, nos dépôts sont loin d'avoir livré toutes les formes intéressantes qu'ils recèlent; leur exploration mérite d'être poursuivie.

La mise en œuvre des matériaux considérables que nous possédons sur les Lichens d'Auvergne, en vue de la publication prochaine d'un travail d'ensemble sur ce vaste groupe de végétaux inférieurs, ne nous permet pas de continuer l'étude attrayante des Diatomées; nous laissons donc à nos confrères en diatomologie le soin de compléter le modeste résultat de nos labeurs.

Les jeunes diatomistes qui marcheront dans la voie que nous avons essayé de leur frayer, n'ont pas à craindre de voir l'attrait du nouveau manquer à leurs recherches ; ainsi que l'a dit le savant abbé Boulay, les œuvres divines, à l'encontre de celles de l'homme qui n'entame que la surface, ont en profondeur des ressources indéfinies; il suffit d'appliquer à un point du domaine scientifique, souvent minime à première vue, la part d'intelligence que nous avons reçue du Créateur pour entrevoir dés merreilles encore inexplorées. 




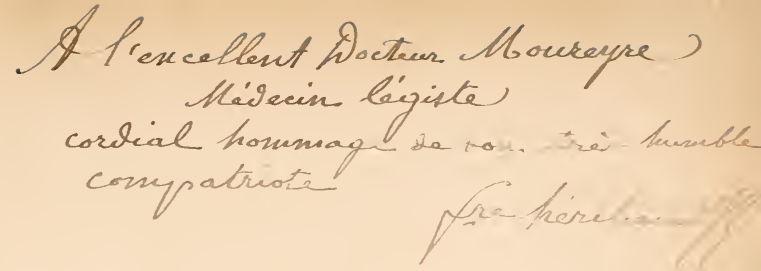

LES DIATOMEES FOSSILES

D'AUVERGNE 


\section{LES}

\section{DIATOMÉES FOSSILES D'AUVERGNE}

(Second Mémoire)

PAR

\section{Le Frère HÉRIBAUD JOSEPH}

1.TUFESEETR AL PRASIONAAT DE CLERMONT-FERRAND

LACRÉAT DE L'ISSTITUT DE FRAXCE

(Académie des Sciences)

MEMURE HOYORAIRE ELL DE L'ACADEMTE DE CLERMOST-FERRAND

ET DE LA SOCIÉTÉ BOTAXTQCE DE FRAXCE

DEO scientiarum Domino

laus et gloria.

AVEC 4 PLANCHES

DESSINÉES PAR LE COMMANDANT MAURICE PERAGALLO

\section{Prix : 10 Francs}

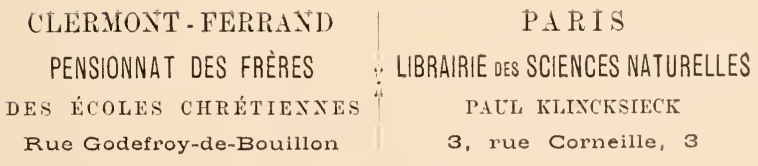

1903 


\section{PRÉFACE}

A près la publication du premier Némoire sur les Diatomées fossiles d'Auvergne (mars 1902), nous espérions pouvoir nous occuper d'un travail d'ensemble sur les Lichens de notre province, par la mise en œurre des matériaux considérables que nous possédons sur ce groupe intéressant de Thallophytes; mais, nous avons dû, grâce aux belles découvertes diatomiques de M. J. PagèsAllary, remettre à plus tard l'étude des Lichens, pour compléter nos recherches sur les Diatomées fossiles du Cantal.

Le résultat a d'ailleurs dépassé nos espérances; dans ce second Mémoire, les diatomistes trouyeront, avec une très belle série d'espèces inédites', l'acquisition de plusieurs faits relatifs à la formation et à la restratification des argiles à Diatomées du Plateau Central, ainsi que l'explication 
très rationnelle de la présence des Diatomées marines et saumâtres qu'elles nous ont lirrées.

Pour les débutants en diatomologie, une bonne épure a été insérée dans le texte, montrant la structure d'un Amphora, et les divers aspects que présente cette Diatomée suivant la position qu'elle occupe dans la préparation.

Les dépôts du Cantal analysés pour la rédaction de ce travail sont au nombre de huit.

Celui de Joursac a été l'objet d'un examen très attentif et plus complet, en raison de l'importance de ses florules diatomique et phanérogamique.

A propos de ce beau dépôt remanié, nous avons démontré et acquis la certitude, que la masse principale, primitivemeut située à 870 mètres d'altitude, s'est détachée du front de la colline, a glissé sur une pente très rapile, et se troure actuellement à 800 mètres d'altitude, un peu au-dessus du village de Pont-du-Vernet.

Il résulte de ce glissement, phénomène assez fréquent dans les pays montagneux, que la stratigraphie de la curieuse montagne de Joursac, bien connue des géologues, exige une légère rectification.

A la suite des dépôts du Cantal, et à titre de 
contribugtion à la flore diatomique du Platean Ceutral, nous avons ajouté la revision des dépôts de la Haute-Loire, trop sommairement étudiés en 1892, et les florules de ceux de l'Ardèche.

Les faits nouveaux pour la science publiés dans notre premier mémoire, ont été l'objet d'un examen critique des plus sévères; or, de l'ensemble de nos observations, il demeure bien établi que les feuilles ne peuvent se fossiliser au cours de la formation d'un dépôt à Diatomées, d'où il résulte que toute masse diatomifère contenant des empreintes de feuilles est un dépôt remanié, restratifié par les eaux; de plus, les florules diattomique et phanérogamique sont absolument independantes l'une de l'autre quant à leur âge géologique; l'âge de la florule phanérogamique correspond exactement à celui de la restratification du dépôt; dans la fossilisation des feuilles la masse diatomique ne joue d'autre rôle que celui de matériaux de sédimentation; ce fait, très important pour la paléobotanique générale est donc bien acquis

La très grande analogie constatée entre les florules des argiles à Diatomées du Cantal, étudiées successivement sour les noms de dépôts de Neussargues, d'Auxillac, de Celles, de Joursac, 
etc., nous autorise à ne voir aujourd'hui dans ces masses diatomifères que des lambeaux de projection d'un dépot initial de formation miocène, lequel ne peut être situé, pour le Cantal, qu’à Chambeuil, entre Murat et le Lioran, c'est-à-dire dans la région où devait s'ouvrir le cratère du grand volcan; c'est de cette région, où l'on constate encore un lambeau du dépôt initial en place, que la masse diatomifère, formée dans des cratères-lacs trachytiques, disloquée par les poussées andésitiques, a été englobée et charriée vers la périphérie du volcan à des distances diverses du dépôt initial; parmi ces lambeaux de projection, il en est deux, Joursac et Andelat, qui ont été repris et restratifiés par les caux, comme le prourent les empreintes de feuilles d'arbres qu'ils renferment.

Les dépôts de Varennes, de La Bourboule et de Perrier (Puy-de-Dôme), synchroniques de ceux du Cantal, ne sont également que des lambeaux remaniés du dépôt initial des Egravats, situé entre la Grande-Cascade du Mont-Dore et la base du Sancy, présentant tous les caractères d'un dépôt en place.

Pour les argiles à Diatomées de la Haute-Loire et de l'Ardèche, contemporaines sûrement des 
dépôts miocènes d'Auvergne, elles n'ont pu se former que dans les cratires-lacs du trachyte inférieur du Mezenc; ces lépôts contiennent tous des empreintes de feuilles, proure évidente que ce sont aussi des lambeaux remaniés par les eaux.

Après aroir exposé notre manière de comprendre la formation des argiles à Diatomées dans des cratères-lacs trachỵtiques, et la présence des espèces marines et saumâtres dans ces argiles, nous avons comparé la flore diatomique miocène du Plateau Centralavec celle de quelques dépôts du nord de l'Europe, du Canada, de plusieurs régions équatoriales, et arec la flore actuelle de Java. L'analogie des volcans trachytiques de Java arec ceux du Plateau Central, domne à la comparaison des deux flores un intérêt tout spécial.

Telles sont les notions, la plupart nouvelles pour la science et pour la préhistoire de notre heau pays d'Auvergne, que l'étude de la flore diatomique de nos argiles miocènes nous a permis d’acquérir.

11 nous reste à prier notre sarant ami, M. le Commandant MI. Peragallo, et nos très distingués compatriotes MII. P. Marty et J. Pagès- 
Allary, d'agréer nos remerciements pour les documents utilisés qu'ils nous ont fournis avec la plus grande complaisance.

Pensionnat des Frères de Clermont-Ferrand, 12 Mai 1903 ,

F. Héribaud Josepit. 


\section{DIATOMÉES FOSSILES}

D'A UVERGNE

\section{DÉPOTS ÉTUDIÉS}

Les dépôts diatomifères d'Auvergne, étudiés pour la publication de ce second mémoire, appartiennent tous au Miocène et au département du Cantal; ce sont les dépôts de Joursac, Andelat, Chambeuil, FraisseBas, Faufouilhoux, Sainte-Anastasie, Moissac, et un afleurement nouveau de celui de Neussargues.

Les Diatomées des gisements de la Haute-Loire, synchroniques de cếux du Cantal, u'étant connues que par le résultat d'un examen très superficiel, publié en 1892, nous avons revu avec soin les dépôts de Ceyssac, de Vals, du Monastier et de la Roche-Lambert.

Enfin, à titre de documents pour la flore diatomique du Plateau Central, nous donnerons, en terminant, l'étude inédite des argiles à Diatomées de Charay, de Ranc, de Gourgouras et de Pourchìres (Ardèche).

\section{I DÉPOTS DU CANTAL}

DÉPOT DE JOURSAC

Ce dépôt remanié est situé entre le torrent du Batein, 
près du village de Joursac, et le hameau de Servière, ì l'altitude de 870 mètres, au N.-E. de la gare de Neussargues, à laquelle il est relié par une bonne route d'environ 5 kilomètres.

Les affleurements se montrent çà et là sur le talus supérieur de la route de Joursac à Servière, sur une longueur de 500 à 600 mètres; le dernier lambean que nous avons observé est tout à fait à proximité de Servière.

Mais ces lambeaux épars, intercalés entre le tuf ponceux et le conglomérat andésitique, le tout recouvert par une puissante nappe de basalte des plateaux, ne constituent qu'une très faible partie du dépôt. A l'occasion d'une excursion à Joursac (septembre 1902), nous a vons constaté que la masse principale s'est détachée du front de la colline, a glissé sur une pente très rapide, et se trouve actuellement à 800 mètres d'altitude, un peu audessus du rillage de Pont-du-Ternet, sur l'Allagnon, comblant le fond d'un petit vallon limité au N. par la butte granulitique de Servière et au S.-O. par le torrent du Batein; le dépôt, aujourd'hui couvert de prairies, repose directement sur la granulite, et est incliné sous un angle d'environ 40 degrés.

C'est cette grande masse diatomique, à surface ondul'́e, qui a été prise d'abord pour une moraine, puis, pour une formation argileuse constituant un horizon stratigraphique.

Le glissement de la partie principale du dépôt de Joursac est un fait rigourensement établi, non seulement par la concordance absolue des lambeaux en place de la partie supérieure, arec les éléments correspondants de la masse inférieure, mais encore par l'identité complète des florules dia to mique et phanérogamique.

Les fouilles que M. Pagès-Allary a eu l'amabilité de 
diriger à notre intention, ont été faites à une cinquantaine de mètres de la cascade du Batein, dans le pré de M. Crégut; c'est donc ì notre excellent compatriote, M. Pagès, et à son aimable collaborateur, que nous devons la plus grande partie des matériaux mis en œuvre pour l'étude diatomique de ce dépôt, et pour la publication prochaine de la Flore miocène de. Toursac.

La masse éboulée est extrêmement riche en plantes fossiles. Le paléobotaniste devra explorer de préférence les blocs de consistance ferme et de couleur brun foncé, non feuilletés; ces blocs présentent des fissures plus ou moins nombreuses, dont les parois sont tapissées de jolis cristaux de sulfate de chanx; les empreintes de feuilles sont dans un état parfait de conservation; c'est dans l'un de ces échantillons que nous avons eu le plaisir de découvrir une fleur de cerisier (Cerasus palceoavium nov. sp.), objet fort rare en paléontologie, montrant encore les étamines dont plusieurs ont le filet couronné par l'anthère.

La partie supérieure du dépôt de Joursac nous était déjà connue par un échantillon reçu en 1891 de notre savant compatriote, M. Marcellin Boule, professeur de Paléontologie au Muséum; mais l'étude de cet échantillon, très pauvre, ne nous livra qu'une dizaine de Diatomées, mentionnées dans notre Mémoire de 1893.

C'est au mois de mai 1902, que M. Pagès-Allary nous adressa deux échantillons provenant de la masse inférieure, et dans lesquels il nous disait avoir constaté des Diatomées; les deux échantillons portaient l'indication : Dépôt de Pont-du-Vernet; comme ils étaient de couleur et de densité différentes, ils furent examinés sous les numéros 1 et 2 .

Le $n^{0} 1$, d'un gris clair, très argileux, ne contenait que des formes déjà connues, et en petit nombre; tan- 
dis que le $n^{\circ} 2$, d'un brun foncé, et portant un fragment de feuille fossile, nous livra une soixantaine de Diatomées fort intéressantes.

Nous avions donc à entreprendre l'étude d'un dépôt très riche, étude longue et laborieuse, comme celle de tous les dépôts remaniés, à cause de l'hétérogénéité de leur masse.

Après quatre mois de recherches minutieuses, nous avons pu établir une liste de plus de deux cents espèces et variétés bien définies, parmi lesquelles près de soixante-dix sont inédites et très remarquables.

De tous les dépôts tertiaires du Plateau Central, celui de Joursac est de beaucoup le plus riche, sous le double rapport de la variété des espèces et du nombre des formes inédites; mais il convient d'ajouter que nul n'a été étudié avec autant de soin et de méthode.

La formation de ce beau dépôt est assurément antérieure aux projections andésitiques du volcan du Cantal, et son remaniement date du Miocène supérieur ou Pontien, correspondant à l'âge de la faune et de la flore phanérogamique, âge bien fixé par MII. Boule et Marty.

Voici la série des Diatomées de Joursac, d'après l'étude d'une centaine d'échantillons et d'environ 200 préparations.

Dans l'énumération des espèces, nous avons observé l'ordre établi dans notre publication récente intitulée : Disposition méthodique des Diatomées d'Aucergne ${ }^{(1)}$.

Rhoicosphenia currata Grun. (Diat. d'Awe, p. 51). Aclenanthes lanceolata Grun. (Diat. d'. Luv., p. 49).

(1) Les Diatomées déji connues sont imprimées en italique, et les espices et varictis nourelles en égygtien. 
Achnanthes joursacense nov. sp. (Pl. XI, fig. 26 et 27 ). - De forme largement elliptique; longueur 15 à $20 \mu$, largeur 8 à $10 \mu$; valves à structure semblable et à cloison en fer à cheval; valve supérieure à pseudo raphé étroitement lancéolé, unilatéralement stauronéiforme, les stries manquant sur l'espace limité par la cloison; stries rayonnantes, courbes, au nombre de 9 en $10 \mu$ au milieu, un peu plus serrées aux extrémités; valve inférieure à aires axiale et centrale nulles; stries courbes, rayonnantes, au nombre de 9 en $10 \mu$ au milieu et un peu plus serrées aux extrémités, non raccourcies ni absentes au milieu de la valve.

Se distingue de l'Aclinanthes lanceolata var. eliiptica Cl., par sa forme plus large, par sa valve inférieure non stauronéiforme, et par le plus grand écartement des stries sur les deux valves.

Cocconeis Placentula Ehrb. (Diat. d'Auv., p. 44).

- lineate Grun. (Diat. d'Auv., p. 44).

- - var. euglypta Grun. (V. H. Syn., pl. 30, fig. 33 et 34 ).

Cocconeis lineata var. rotunda nor. - Diffère du type par sa forme suborbiculaire; longueur $35 \mu$, largeur $30 \mu$.

Navicula Pupula Ktz. (Diat. d'Auv., p. 118).

-- - var. minuta V.-H. (Diat. d'Auv., p. 118).

- Bacillum Ehrb. (Diat. d'Aue, p. 117).

- - var. minor V.-H. (Diat. d'Auv., p. 117).

-- pseudo-Bacillum Grun. (Diat.d'Aur.,p.118)

Navicula pseudo-Bacillum var. elapsa nov. (Pl. IX, fig. 30). - Se distingue du type par sa forme plus allongée, par son aire hyaline axiale plus 
large, non brusquement arrondie autour du nodule médian, par le raphé et le nodule médian très fins, à peine visibles, tandis que les nodules terminaux et leurs appendices latéraux sont nettement marqués et allongés.

Navicula ventricosa Ktz. (T. H. Synn., pl. 12, fig. 19).

Navicula ventricosa var. decrescens noc. (Pl. XI, fig. 9). - Valve décroissant de largeur par de faibles ondulations jusqu'à ses extrémités largement arrondies; longueur 60 à $70 \mu$, largeur au nodule médian 11 à $12 \mu$; aréa stauronéiforme linéaire ; stries au nombre de 18 en $10 \mu$, perpendiculaires au raphé, légèrement courbes au milieu de la valve et rayonnantes aux extrémités.

Diffère du type principalement par ses extrémités diminuées au lieu d'être dilatées.

Naricula amphirhynchus Ehrb. (Diat.d'Aur.,p.115).

- firma Ktz. (Diat. d'Auv., p. 116).

- amphigomphus Ehrb. (Diat.d'Auv., p. 113).

Navicula dubitata nor. $s p$. (Pl. IX, fig. 28). - Talve elliptique, à extrémités légèrement acuminées; longueur $50 \mu$, largeur $17 \mu$; semblable au Nacicula laterittata Pant. (Pantocseck, Ung. III, pl. 8, fig. 122); même disposition des stries et même écartement, 18 en $10 \mu$, que dans l'espèce de Pantocseck, d'après Clève (Syn. 1891, p. 67); pourrait être prise pour une petite forme de cette espèce si elle n'en différait, çu moins d'après le dessin de Pantocseck, par le raphé qui est entouré de bourrelets, et dont le nodule central est beaucoup plus petit et non entouré d'une aréa circulaire; ces caractères différentiels ne permettent pas de confondre les deux formes. 
Naricula cuspidata Ǩtz. (Diat. d’Aur:, p. 107). sculpta Ehrb. (Diat. foss. d'Auv., p. 41).

Navicula Gendrei nor. sp. (Pl. IX, fig. 31). - De forme lenticulaire, à extrémités prolongées, fines et arrondies; longueur de la valve 40 à $55 \mu$, largeur 15 à $18 \mu$; aire hyaline axiale trìs étroite, lancéolée, quelquefois un peu stauronéiforme au nodule médian; stries fines, formées de petits points ronds, dont l'écartement diminue, dn raphé vers le bord de la valve; les stries sont uniformément distancées aux extrémités et au centre de la valve, $9{ }^{1}{ }_{2}$ ¿̀ 10 en $10 \mu$, et à peu près également inclinées sur le raphé; l'espace triangulaire laissé par les deux stries les plus longues de chaque côté, et arrivant près du nodule médian, est occupé par trois ou quatre stries ordinairement de longueur décroissante et parallèles anx stries de l'un des côtés.

Pantocseck figure dans ses Diatomées fossiles de IIongrie (Ung. III, pl. 9, fig. 194), une forme du Naricula styriaca Grun. ( Nav amplitbola Cl.) qui présente la même disposition des stries centrales, mais la Diatomée de Joursac diffère de celle de Hongrie par sa forme extérieure et la striation générale de la valve; les deux Diatomées ne peurent être identifiées.

Cette espèce est dédiée ì M. Ch. Le Gendre, le savant Directeur de la "Revue Scientifique ) et de la "Société botunique du Limousin. )

Naricula lanceolata Ktz. (Diat. d'Auv., p. 103).

- gastrum Donk. (Diat. d'Aur., p. 102).

- - var. major (Diat. d'Aue., p. 102).

- Placentula Ehrb. (Diat. d'Aluv., p. 102).

- anglica Ralfs. (Jiat. d'Aur., p. 102).

Navicula Corbieri nov. sp. (Pl. IX, fig. 16). 
- Talve elliptique, à extrémités coniques et très légèrement rostrées; longueur $30 \mu$, largeur $10 \mu$; nodules terminaux petits mais bien marqués, logés dans la partié rostrée terminale qui est très brillante et donne un aspect particulier à la valve; aire hyaline axiale très étroite aux extrémités, et s'élargissant progressivement en losange autour du nodule médian; stries au nombre de 10 en $10 \mu$; de chaque côté trois stries centrales sont perpendiculaires au raphé, celle du milieu plus longue que les deux voisines, elles forment un triangle sur lequel viennent s'appuyer les stries suivantes, celles-ci, d'abord rayonnantes, se redressent peu à peu, mais ne deviennent pas convergentes.

Cette espèce, bien distincte, est dédiée à M. le professeur L. Corbière, auteur de la Nourelle Flore de Normandie, et de plusieurs publications bryologiques très estimées.

Navicula joursacensis not. sp. (Pl. IX, fig. 15). - Talve elliptique, à extrémités arrondies et un peu coniques; longueur $40 \mu$, largeur $16 \mu$; raphé bifide, analogue à celui du Stuuroneis Phenicenteron; nodules terminaux arrondis, bien visibles; aire hyaline axiale nulle, la centrale petite et arroudie; stries au nombre de 12 en $10 \mu$, non distinctement granulées, convergentes et courbes, les médianes non alternativement longues et courtes.

Navicula Reinleardtii Grun. (Diat. d'Aur., p. 102).

Navicula Reinhardtii var. elliptica nov.C'est la forme représentée par Van-Heurck, Syn. pl. 7, fig. 6 , que nous trokirons à Joursac, et à laquelle nous avons cru utile de donner un nom. 
Navicula triangulifera nov. sp. (Pl. IX, fig. 20). - De forme analogue au Navicula digitoradiata Greg. (V.-H. Synn., pl. 7, fig. 4), comme dans l'espèce de Gregory les stries sont raliantes au centre et convergentes vers les extrémités; la longueur de la valve varie à peu près dans les mêmes limites, 55 ì $60 \mu$; en diffère par l'aire axiale qui est dilatée au centre en forme de losange; par las stries des extrémités plus serrées $(12$ en $10 \mu)$ que celles dı milieu $(9$ en $10 \mu)$; par la disposition très particulière et caractéristique des cinq stries médianes, dont l'ensemble forme un triangle encadré par les deux stries suivantes qui sont t ès longues et fortement inclinées; le reste de la striation est tout à fait analogue à la striation du Navicula digito-radiata Greg.

Naricula digito-radiata Greg. (Diat. d'Awv., p. 25).

Navicula digito-radiata var. obesa nov. (Pl. XI, fig. 16). - Valve largement lancéolée; longueur 45 à $55 \mu$, largeur 12 à $15 \mu$; stries au nombre de 8 en $10 \mu$ au milieu, plus serrées aux extrémités, où elles sont perpendiculaires au raphé.

Differe du type par sa grande largeur proportionnellement à sa longueur.

Navicula Costei nov. sp. (Pl. IX, fig. 17). Valve de forme elliptique lancéolée, à extrèmités largement arrondies et non capitées; longueur 120 à $160 \mu$, largeur, au nodule central, 20 à $25 \mu$; stries très radiantes jusqu'aux extrémités, oì elles présentent le genouillement caractéristique du Tavicula oblonga Ktz., au nombre de 9 en $10 \mu$, les centrales alternativement longues et courtes, et par conséquent n'étant pas notablement plus écartées que les autres; aire hyaline axiale bilaté- 
rale, s'élargissant autour du nodule central en aréa circulaire.

Se distingue du Naricula oblinga Ǩtz. par sa longueur moindre, par sa forme plus lancéolée, par ses stries plus serrées, présentant, surtout autour du nodule central, des stries alternativement longues et courtes.

Nous dédions cette belle Naricule à M. le chanoine H. Coste, l'éminent auteur de la Flore descriptice et illustrie de la France, en cours de publication.

Navicula Costei var. bacillaris not. (Pl. IX, fig. 19'. - Differe du type par sa forme bacillaire, par sa taille beaucoup plus petite; valve ayant à peine $75 \mu$ de longueur et $12 \mu$ de largeur; extrémités largement arrontlies.

A. Schmidt représente (At)., pl. 77, fig. 6̈̈), une variété semblalle au Nar. oblonga Ktz., mais son dessin ne montre pas de stries médianes alternativement longues et courtes, ce qui ne permet pas d'identifier les deux formes.

Lacicula radiosa Kitz. (Diat. d'Aur., p. 99).

- - var. acuta Grun. (Diat. d'Aur., p. 99).

- Hunncocepllala Ktz. (Diat. d'Auv., p. 101).

- borealis Ktz. (Diat. d'Aur., p. 86).

-. mpestris Ǩtz. (Diut. d'Alue., p. 84).

- nobitis Ehrb. (Diat. d'Aur., p. 80).

-- major Ktz. (Diat. d'Auv., p. 82).

Navicula major var. convergentissima nov. (Pl. IX, fig. 111). - Cette belle variété du Nacicula major est de grande taille; longueur $210 \mu$; elle est caractérisée par la forte convergence et la divergence des côtes, qui sont courbées et ondulées; raphé délicat et peu visible. 
Navicula cincta Ktz. (Diat. d'Auv., p. 98).

- Heufleri Grun. (Diat. d'Auv., p. 98).

- meniscul"nsSchum.(V.-H. Syn., pl.8, fig. 20).

- Esox Ehrb. (Diat. d'Auv., Pl. IV, fig. 4).

Navicula Esox Ehrb. var. recta nov. (Pl. IX, fig. 22). - Longueur 125u, largeur $20 \mu$, à flanes rectilignes, à extrémités cunéiformes et largement arrondies; raphé simple, aréa large, fusiforme, occupant un tiers de la surface de la valve, largement arrondie autour des nodules terminaux et médian, mais inégalement développée autour de ce dernier; côtes rayonnantes au centre et fortement convergentes aux extrémités, au nombre de 7 en $10 \mu$.

Diffère du type par sa forme générale (Diat. d'Auv., Pl. IV, fig. 4) et par le nombre de ses stries. (Clève, Syn. 1895, p. 90).

Navicula Olivieri nov. sp. (Pl. IX, fig. 23). De taille moyenne et trapue; longueur de la valye 85 à $87 \mu$, largeur 19 à $20 \mu$, presque bacillaire, à extrémités coniques et très largement arrondies; aire hyaline axiale assez large, inégalement dilatée autour du nodule médian, d'un côté iortement sur une petite longueur et en demi cercle, de l'autre côté un peu moins mais sur une plus grande longueur; raphé simple, délicat, à nodules terminaux aplatis; côtes radiantes au milieu, convergentrs aux extrémités, séparées les unes des autres et d'écartement très variable, en movenne $7 \frac{1}{2}$ en $10 \mu$.

Ressemble, à première vue, à notre Nacicula Esox var. recta qui serait très écourté, porportionnellement à sa largeur, mais en diffère par son aréa, par ses nodules aplatis et par sa striation.

Nous dédions cette Navicule au savant Directeur et 
fondateur de la Revue scientifique du Bourbonnais, à II. Ernest Olivier.

Navicula decurrens Elırb. var. curtecos. tata nov. (Pl. IX, fig. 27). - De forme bacillaire, à extrémités atténuées et largement arrondies; longueur de la valve $65 \mu$, largeur $11 \mu$; aréa large, arrondie autour des nodules médian et terminaux; côtes au nombre de 10 en $10 \mu$, convergentes anx extrémités, et radiantes au centre, où elles sont trìs courtes, et manquent même quelquefois.

A. Schmidt (Atl., pl. 45, fig. 30), a dessiné, sous le nom de Pimmlaria decurrens, une forme semblable, mais qui est moins bacillaire, et l'aréa est beaucoup plus petite.

Navicula Leveillei nor. sp. (Pl. IX, fig. 21). De forme pinnulariće bacillaire, à centre légèrement élargi, et à extrémités un peu coniqnes, largement arrondies; longueur de la valve $105 \mu$, largeur $15 \mu$; aire hyaline axiale assez large, fortement arrondie autour du 1.odule médian, et même stauronéiforme par l'absence des stries médianes de l'un ou des deux côtés, très rétrécie avant les extrémités, où les côtes touchent le raphé, très visible à cet endroit; nodules terminaux arrondis et très grands; côtes non distinctement granulées, radiantes au milieu de la valve, très convergentes anx extrémités et légèrement courbées, au nombre de 10 en $10 \mu$.

Se distingue du Navicula Esox Ehrb., arec lequel il a quelque analogie, par sa taille plus petite, par la forme et la disposition des côtes, qui sont plus fines, isolées, plus serrées, et par leur interruption au milieu de la valie. 
Cette espèce, très distincte, est dédiée à notre savant ami, MI. H. Léveillé, Secrétaire perpétuel de l'Académie internationate de Gíograplie botanique.

Naricula Brebissonii Kitz. (Diat. d'Lur., p. 88).

- mesolepta Ehrb. (Diat. d'Auv., p. 96).

-- -var.stauroneiformis Gr.(Diat.d' Aur.,p. 96)

- gracillima Pritch. (Diat. d'Au:, p. 87)

Navicula gracillima var. lucida noc. - Se distingue du type par les aires hyalines axiale et centrale beaucoup plus larges.

Taricula mesotyla Ehrb. (Diat. d'Aur., p. 97).

- RenauldiF. H. (Diat. foss.d'Auv., H.III, fig. 9)

Navicula Renauldi var. major. noı. (Pl. XII, fig. 4). - Tout à fait conforme au type comme contour extérieur, mais plus grand et plus largement strié; longueur de la valve $50 \mu$; stries au nombre de 8 en $10 \mu$.

Navicula amphibola Clève (Syn. 1895, p. 45). - arema (Diat. d'Aur., Pl. IV, fig. 19).

- - var.Stauroneiformis (Diat.foss.d'. Aur., Pl. VII, fig. 10).

Navicula Berriati nox. sp. (Pl. IX, fig. 24). Frustule suborbiculaire; longueur $28 \mu$, largeur $18 \mu$; raphé délicat mais bien visible, ¿̀ extrémités centrales éloignées l'une de l'autre, nodules terminaux notablement éloignés du bord de la valve; aire hyaline axiale fusiforme, non brusquement élargie autour du nodule central; stries formées de petits points fins mais très distincts, progressivement rayonnantes, et plus serrées aux extrémités qu'au milieu de la valve, où elles sont au nombre de 6 en $10 \mu$; entre les stries principales, on en 
voit d'autres très courtes, tout ì fait marginales, et dont nous n'avons pas tenu compte pour la mesure de l'écartement.

Diffère du Nariculu scutelloides IT. Sm., par sa taille plus petite; par les stries moins serrées et n'atteignant pas le raphé, Jaissant au contraire une aire hyaline axiale fusiforme très nette.

Cette jolie Navicule est dédiće à M. Berriat SaintI'rix, magistrat honoraire et Docteur en Droit.

Navicula Berriati var. minor. nov. (Pl. IX, fig. 25). - Se distingue du type par sa forme plus ronde, par sa taille beaucoup plus petite, longueur 15 à $18 \mu$, largeur 12 à $14 \mu$, et par la striation un peu plus serrée.

Stauroneis Pluenicenteron Ehrb. (Diat, d'Aux., p. 75. ) - Smithï Thw. (Diat. d'Auv., p. 79).

Stauroneis quadrata nor. $s p$. (Pl. IX, fig. 32). - Très petit, longueur $15 \mu$, largeur $8 \mu$; face valvaire carrée, à angles arrondis et à extrémités restrées non capitées; stauros linéaire, étroit; stries invisibles dans le baume. Petite espèce curiense et très distincte.

Diproneis elliptica Clève. (Diat. d'Aur., p. 104).

- - var.minutissimaGr. (Diat.d'Auv.,p. 10").

- Pagesi (F. Hérib.) (Diat. foss. d'Aur., Pl. IX. fig. 7).

Amplora lityjca Ehrb. (Diat. d'Aur., p. 63).

- oralis Ehrb. (Diat. d'Aur., p. 62).

Amphora affinis Ktz. (V. H. Syn., pl. 1, fig. 2).

- Pediculus Grun. (Diat. d'Aur., p. 63).

- - var. major Grun. (Diat. d'Aur., p. 63). 
Amphora distincta nox. sp. (I']. IX, fig. 2). Espèce de petite taille; longueur 30 à $35 \mu$; face valvaire cymbiforme, à extrénités furtement prolongées et arrondies ; raphé arqué, assez éloigné de la face ventrale; aire hyaline axiale nulle, aire centrale formant un pseudo-stauros des deux côtés; du côté dorsal, ce pseudo-stauros, arrivé un peu avant le bord dorsal, s'élargit en 'T, dont les branches, relativement assez larges et étendues, divisent les stries en deux faisceaux; stries indistinctement granulées, au nombre de $13 \mathrm{en}$ $10 \mu$. La face ventrale porte des deux côtés du pseudostauros évasé, des stries fortement convergentes.

Diffère d'une petite forme de l'A mplenralityca Ehrb. (4. oralis Elırb. var.) par le grand développement de la face ventrale, qui est fortement striée, et par la plus grande dimension de l'épanchement en T du pseudustauros dorsal. - Cette petite espèce, très distincte, se trouve aussi dans le dépôt de Moissac.

Cymbella Harioti nov. sp. (Pl. IX, fig. 8). Espèce assez petite; longueur $60 \mu$, largeur $10 \mu$, côté dorsal gibbeux, côté ventral légèrement et régulic̀rement convexe; ressemble, comme forme générale ì notre Cymbella radiosa, mais en diffère nettement par sa striation; raphé légèrement courbé; nodules petits ; stries radiantes, marginales, laissant une aire hyaline axiale large, arrondie autour du nodule médian, an nombre de 9 à 10 en $10 \mu$ à la partie dorsale et de 10 ì 11 en $10 \mu$ à la partie ventrale.

Ce Cymbella est dédié à MI. Paul Hariot, Assistant an Muséum.

Cymbella radiosa nor. sp. (Pl. IX, fig. 13). Valve à extrémités longuement prolongées et recurvées 
du côté dorsal ; longueur $62 \mu$, largeur $10 \mu$; striation du Navicula rartiosa, c'est-ì-dire stries centrales alternatirement courtes et longues, pour rendre les suivantes rayonnantes, se redressant ensuite progressivement pour devenir convergentes aux extrémités; les centrales sont au nombre de 10 en $10 \mu$ à la partie dorsale et de $11^{1} / 2$ en $10 \mu$ à la partie ventrale; celles des extrémités sont un peu plus serrées.

Cymbella cymbiformis Ehrb. (Diat. d'Aux., p. 64).

Cymbella hungarica (Grun.) Pant. (Ung. II, pl. 1, fig. 14). - Tout à fait conforme à la fig. 17, pl. 10 de l'Atl. de A. Schmidt, présentant la même sinuosité du raphé près du nodule médian.

Cymbella Brevieri nox. sp. (Pl. IX, fig 12). Taille moyenne; longueur 70 à $80 \mu$, très peu cymbiforme, côté dorsal arqué, côté ventral très légèrement bombé, extrémités larges, arrondies, presque tronquées; raphé occupant le milieu de la valve, régulièrement arqué; riodule central petit, rond, bien visible ì un faible grossissement; nodules terminaux recurvés vers la face dorsale, assez gros, virguliformes; aires hyalines axiale et centrale nulles, les striєs aboutissant très près du raphé et entourant les nodules; stries fortes, radiantes, formées par des séries de petites lignes parallèles au raphé, au nombre de $S$ en $10 \mu$ à la partie dorsale, et 10 en $10 \mu$ à la partie ventrale.

Cette espèce, bien distincte, est dédiée à notre très distingué corespondant, M. Brevière, botaniste à Ambert.

Cymbella Foucaudi nox. sp. (Pl. IX, fig. 7). - De forme massive, à extrémités pointues; longueur 
de la valıe $60 \mu$, largeur $16 \mu$, côté dorsal régulièrement et fortement arqué, côté ventral droit ou légèrement arqué et gibbeux an milieu; raphé fortement arqué, placé au milieu de la valve; nodules terminaux petits, placés tout à fait au sommet de la valve; aire lyyaline axiale s'élargissant progressivement des extrémités au milieu, où elle n'est brusquenent élargi que du côté ventral du nodule médian; stries obscurément granulées, régulièrement radiantes, un peu plus serrées aux extrémités qu'au milieu, oì elles ont l'écartement de $6 \frac{1}{2}$ en $10 \mu$ ì la partie dorsale et de 8 en $10 \mu$ à la partie ventrale.

Ressemble ì notre Cymbella conifera dont les extrémités n'auraient pas été raccourcies coniquement. Diffère absolument de certains Encyonema, dont il rappelle la physionomie, par la forme de ses nodules terminaux petits, ronds, et placés tout ì fait aux extrémités de la valve.

Nous dédions ce Cymbella à M. Foucaud, botaniste à Rochefort-sur-ILer.

Cymbella P'agesi F. II. (Diat.foss. d'Aur., Pl.VII, fig.7)

Cymbella Creguti nov. sp. (Pl. IX, fig. 3). Longueur de la valve $52 \mu$, largeur $17 \mu$; côté dorsal fortement arqué, gibbeux; côté ventral légèrement Lombé; extrémités prolongées et arrondies; raphé très arqué, placé sensiblement au milieu de la valve, non récurré du côté de la face dorsale; nodules terminaux petits, ronds, bien visibles, placés au centre de l'arrondi terminal de la valve; nodule central petit; aire hyaline axiale nulle, la centrale très petite, arrondie seulement du côté ventral; stries radiantes, fortes, distinctement granulées, au nombre de $9 \frac{1}{2}$ en $10 \mu$ à la partie dorsale et de 11 en $10 \mu$ à la partie ventrale. 
Diffère du Cymbella tumida Bréb. par ses extrémités plus fines, par ses nodules terminaux non récurvés du côté de la face dorsale, et par sa striation plus serrée.

Nous dédions ce joli Cymbella à II. Crégut, propriétaire de la principale partie éboulée du dépôt de Joursac, en reconnaissance des services précieux qu'il nous a rendus arec la plus parfaite amabilité.

C'ymbella lanceolata Ehrb. (Diat. d'Auv., p. 68).

- aspera Ehrb. (Thiat. d'Aur., p. 69).

- turgitula Grun. (Diat. d'A Lur., p. 68).

- Ehrenbergï Greg. (Diut. d'Aur., p. 6t).

- - var. minor T.-H. (Diat. d'Aur., p. 65).

- affinis Ktz. (Diat. d'Aur., p. 66).

Cymbella meniscus nox:sp. (Pl. IX, fig. 5). - Petite espèce de forme lenticulaire, ¿̀ extrémités arrondies et très légèrement rostrées; longueur $43 \mu$, largeur $18 \mu$; aspect et striation du Naricula Placentula var. $\operatorname{minor}($ V. H. Synn., pl. 8, fig. 26); raphé droit, aires hyalines axiale et centrale presque nulles; stries rayonnantes, non distinctement granulées, un peu plus serrées aux extrémités qu'au milieu de la valve, où elles sont au nombre de 9 en $10 \mu$ du côté dorsal, et $10 \%$ à 11 en $10 \mu$ du côté ventral.

A. Schmidt (Atl. pl. 71, fig. 75), donne, sous le nom de Cymbella americana var. acuta A. Sch., une espèce qui ressemble assez bien à celle de Joursac; mais la Diatomée américaine s'en distingue par sa taille plus grande et par son mode de striation.

Cymbella Laubyi nov. sp. (P]. IX, fig. 4) Frustule subnaviculaire elliptique, à extrémités rostrées, non capitées et arrondies; longueur de la valve 50 ì $65 \mu$, largeur 15 à $20 \mu$; raphé presque rectiligne, à no- 
dules terminaux placés très près des bords de la valve; aire hyaline axiale presque nulle, s'élargissant en losange autour du nodule médian; stries rayonnantes, les ventrales plus serrées que les dorsales, et des deux côtés plus serrées aux extrémités qu'au milieu, oì elles sont au nombre de 9 en $10 \mu$ à la partie dorsale et de $10 \%$ en $10 \mu$ à la partie ventrale.

Nous dédions ce Cymbella à II. Lauby, préparateur à la Faculté des Sciences de Clermont, en souvenir des documents qu'il a eu l'amabilité de nous procurer.

Cymbelia leptoceras Kitz. (Diat. d'Auv., p. 66).

Encyonema crespitosum Kitz. (Diat. d'Au', 1. 73).

Encyonema cæspitosum Ktz. var. Auerswaldii V. H. -- Tout à fait conforme ì la fig. 14, pl. 3 du Synopsis de Van-Henrck.

Encyonema intermedium nox. sp. (Pl. XI, fig. 5). - Longueur de la valre 30 à $40 \mu$, côté dorsal très courbé, côté ventral droit, avec le centre gibbeux et les extrémités prolongées et courbées rers la face ventrale; aire axiale presque nulle du côté dorsal, nettement marquée du côté ventral, élargie autour du nodule central, principalemerit du côté ventral; stries indistinctement granulées, au nomlire de 9 en $10 \mu$ ì la partie ventrale et au milieu de la partie dorsale, plus serrées aux extrémités de la valve; nodule central du raphé à extrémités légèrement récurvées vers le bord dorsal, nodules terminaux entourés par les stries de la partie ventrale, placés assez loin des extrémités, et très rapprochés du bord dorsal, où ils se prolongent en virgule vers les extrémités de la valve.

Diatomée intermédiaire entre l'Encyonema cespito- 
sum Ktz. et l'Encyonema prostratum Ralfs. A. Schmidt figure dans son Atlas, pl. 10, fig. 60-62, trois formes analogues qui n'en diffèrent que par la partie ventrale de l'aréa centrale; il ne les nomme pas.

Encyonema Grandi nor. sp. (Pl. IX, fig. 9). - De taille assez petite, longueur $60 \mu$, largeur $14 \mu$; côté dorsal fortement et régulièrement courbé, côté ventral rectiligne, légèrement renflé au milieu; raphé droit, à nodules terminaux virguliformes, appliqués contre le bord dorsal; au nodule central, les deux extrémités du raphé sont légèrement infléchies rers le bord dorsal; aire hyaline axiale presque nulle du côté ventral, assez large an contraire du côté dorsal, non dilatće autour du nodule médian; côte médiane du côté dorsal plus longue que ses voisines, et s'allongeant presque jusqu'au niveau des deux extrémités du raphé; côtes très nettes, quoique fines et isolées, non distinctement divisćes en travers, régulièrement rayonnantes à la partie dorsale, presque parallèles à la partie ventrale, un peu plus serrées aux extrémités qu'au milieu, où elles sont au nombre de 6 en $10 \mu$ à la partie dorsale et de 7 en $10 \mu$ à la partie ventrale.

Ressemble à la fig. 51, pl. 10 de l'Atlas de A. Schmidt, nommé Encyonema turgidum Grun., mais notre $C_{y m}$ bella en diffère par l'aire hyaline centrale non dilatée, et par sa striation.

Nous dédions cette bonne espèce au savant botaniste de Bourges, MI. A. Le Grand.

Gompleonema Tibrio Ehrb. (Diat. d'Aur., p. 59).

Gomphonema latestriata nov. $s p$. (Pl. X, fig. 5). - De grandeur moyenne; longueur $72 \mu$, largeur $11 \mu$, frustule formé de deux parties coniques, 
réunies par un arrondi; valve à extrémités arrondies; raphé et nodules très faibles, à peine visibles; aire hyaline axiale notable, presque aussi large aux extrémités qu'au milieu; stries faiblement radiantes, au nombre de 9 en $10 \mu$, excepté pour les trois stries médianes qui sont beaucoup plus écartées, et dont l'une est un peu écourtée pour donner place à un point unilatéral situé presque sur la ligne de terminaison des stries; la strie médiane de l'autre côte est beaucoup plus écourtée, presque marginale.

Se distingue du Gomphonema Vibrio, dont il a la physionomie, en ce que les flancs de la valve, formant les extrémités inférieure et supérieure, sont plutột bombés que concaves; l'aire hyaliue axiale est aussi plus large et les stries plus serrées.

Gomphonema intricatum K'tz. (Diat. d'Auv., p. 57).

- ～- var. pumila Gr. (Diat.d'Auv.,p. 57.

- subcluratum Grun. (Diat.d'Aur., p. 55)

- _ -var.acuminata (Diat.d'Aur.,pl.3, fig., 8)

- micropus Ktz. (Diat. d’Aur., p. 5\%).

Gomphonema micropus var. major nov. -- Bien conforme, pour la striation, à la fig. 46, pl. 24 du Synopsis de Tan-Heurck, mais beaucoup plus grand qu'il n'est indiqué par Clève (Syn. 1894, p. 180); longueur de la valve $40 \mu$.

Gomphonema commutatum Grun. (Diat. c'Auv.,p.55).

Gomph. eriense Grun. (V.H. synn., pl. 23, fig. 10).

Gomphonema eriense var. acuminata nor. (Pl. XII, fig. 10). - Longueur 45 à $55 \mu$, largeur 12 à $14 \mu$, largement lancéolé, à extrémités prolongées, sur- 
tout l'inférieure, qui est relativement étroite $(3 \mu)$ et non dilatée; stries au nombre de 11 en $10 \mu$, excepté au centre où l'écartement est de 8 en $10 \mu$.

Diffère du type par ses extrémités plus aiguës et plus prolongées, par sa taille plus grande et par ses stries plus écartées.

Gomplonema acuminatum Grun. (Diat. d'Auv., p. 53)

- Augur Elirb. (Diat. d'Auv., p. 54).

- insigne Greg. (Diat. d'Aue., p. 59).

Gomphonema insigne var. minor Grun. Identique à la fig. 14, pl. 24, du Syn. de Tan-Heurck.

Gomph. semiapertum Gr. (V. H. Syn. pl. 24 , fig. 42).

- - var. TergestinaGr. (Diat.d'Aur.,p. 61).

Gomphonema exscissum nov. sp. (Pl. X, fig. 6). - De forme générale conique, à extrémités arrondies et à centre renflé; longneur de la valve 50 à $60 \mu$, largeur au centre 8 à $10 \mu$; aire hyaline axiale étroite, élargie légèrement autour du nodule médian, et dilatée latéralement d'un seul côté par l'absence des stries médianes; stries plus serrées à l'extrémité inférieure $(11$ à 12 en $10 \mu)$ qu'à l'extrémité supérieure $9 \frac{1}{2}$ à 10 en $\left.10 \mu\right)$; absence de point unilatéral; silice robuste.

Gomphonema parvum not: sp. (Pl. X, fig. 4). - Petite espèce assez semblable au Gomphonema intricatum var. pumila de Van-Heurck (Sym pl. 24, fig. 35), mais plus grand, longueur de la valve $38 \mu$, largeur $8 \mu$; s'en distingue en outre par son aire hyaline 
axiale plus large, par son raphé plus fin, son nodule central presque invisible, par ses nodules terminaux, au contraire, lien marqués et éloignés des extrémités, caractères opposés à ceux de la figure désignée; stries presque marginales, au nombre de 10 en $10 \mu$ à la partie supérieure et de 13 en $10 \mu$ à la partie inférieure, mais plus serrées vers les deux sommets de la valve.

Gomphonema accessum nov. sp. (Pl. X, fig. 2 et 3 ). - De forme presque naviculaire rhom. boïlale allongée, flancs légìrement concayes, extrémités arrondies; longueur 50 ¿ $70 \mu$, largeur 8 ¿ $12 \mu$; aire hyvaline axiale notable, un peu resserrée au milieu de la valve, où elle s'arrondit autour du nodule médian; raphé à peine visille, nodules très petits, pas de point unilatéral visible; stries presque parallèles, au nombre de 13 en $10 \mu$, un peu plus serrées à l'extrémité inférieure.

Differe du Gomphonema intricatum Grun. (V. H. Syn. pl. 24, fig. 28), dont il a ì peu près la forme, par sa striation plus fine, non rajonnante, et moins sensiblement écourtée autour du nod:le médian.

Gomphonema Gilloti nov. sp. (H. X, fig. i). - Talve elliptique, dont une extrémité est prolongée pour former la partie inférieure du frustule; longueur $50 \mu$, largeur au nodule médian $12 \mu$; raphé et nodu'es centraux très faibles, nodules terminaux petits, mais bien visibles; point unilatéral nul ou invisible; aire hyaline axiale bien marquée, insensiblement élargie autour du nodule médian; stries légèrement rayonnantes, non distinctement granulées, la médiane de l'un des côtés légèrement écourtée, au nombre de 11 en $10 \mu$ à la partie supérieure, et de 12 en $10 \mu$ à la partie inférieure, les médianes n'étant pas sensiblement plus écartées que les voisines. 
Se distingue du Gompleonema affine Kitz. (T. H. Syn., pl. 24, fig. 9), dont il a la forme, par son aire hraline axiale plus large, par l'absence du point unilatéral, par ses stries non granulées et moins serrées.

Cee Gomphonema est dédié ì M. le Docteur Gillot, botaniste à Autun.

Gomphonema arcticum Grun. - Espèce marine des mers du Nord, bien conforme it la fig. 30, pl. 25 du Synopsis de Tan-Heurcl.

Gomphonema Licmophoraoides nor. sp. (Pl. X, fig. S). - De taille assez petite; la partie supérieure de la valve est de forme elliptique, largement arrondie, flancs presque rectilignes, se rapprochant subitement pour former la partie inférieure qui est fortement prolongée, atténuée, bacillaire et arrondie: longueur 40 à $45 \mu$, largeur de la partie supérieure $10 \mu$, largeur de la partie inférieure $\check{\jmath} \mu$; aires lỵalines axiale et centrale nulles, pas de point unilatéral; stries parallèles, non perlées, au nombre de 9 en $10 \mu$.

Notre Gomphonema de Joursac rappelle la physionomie du Licmophora ocate var. barbadensis Grun (V. H. S.yn. pl. 47, fig. 13, des îles Barbades.

Gompleonema exiguem Kitz. Diat. d’Aur., p. 61). abbreviatem K'tz. Diut.d'.1ur., p.61\%.

Symedra capituta Ehrb. (Diat. d'A Lur., p. 139).

- C'ha Elırb. Diat. d'Aue, p. 137).

Synedra joursacensis $n x . s p$. (Pl. XII, fig. S). - Semblable à la variété spahmulifera du Synnedra Clna (V. H. Sym. pl. 20, fig. 4), mais dont r'élargissement en spatule est plus progressif, plus grand, ter- 
miné par un rostre fin et plus long, analogue à celui du Synedra Clna rar. ritrea; stries au nombre de 8 en $10 \mu$, interrompues an milieu de la valve, non distinctement granulées, laissant entre elles un pseudo-raphé fin, mais très visible ; face connective retrécie au milieu et à extrémités très fortement dilatées en éventail.

Fragilaria brevistriate Grun. (Diat. d'Aur., p. 146). - - var.pusilla Grun. (Diat. d'Aur., p. L'́í). - lapponica Grun. (Diat. foss. d'.1ux.,p. 21).

Fragilaria Harrisonii Grun. var. major nov. (Pl. X, fig. 12). - Se distingue du type (Tan-Heurck Symopsis, l, 45 , fig. 28), par sa taille beaucoup plus grande; la forme dessinée par Van-Heurck atteint à peine $20 \mu$, alors que celle de Joursac a une longueur de 35 à $40 \mu$; notre rariété major doit représenter probablement l'une des dernières variations du type dans le sens ascendant, et c'est pourquoi il nous a paru intéressant de la publier.

Fragilaria Gustavei nov. sp. (Pl. X, fig. 13). - Petite espèce de 20 à $30 \mu$ de longueur et de 8 à $10 \mu$ de largeur; face valraire renflée, à extrémités atténuées et largement arrondies, semblable au Fragilaria construens var. venter (V. H. Sym. pl. 45, fig. 26), mais beaucoup plus grand, et à stries plus écartées; stries au nombre de 13 en $10 \mu$ au milieu de la ralre, rayonnantes et d'intensité décroissante des bords de la valve au milieu, où elles disparaissent insensiblement sans que l'on puisse apprécier la largeur du pseudo-raphé.

Fragilaire dédiée à l'auteur de l'excellente Clef analytique de la Flore d'Aurergne (1873), au très regretté Frère Gustave, l'un des botanistes qui ont le plus contribué à la connaissance de notre flore locale. 
Fragilaria construens Grun. (Diat. d'Aur., p. 143). - - var.center Grun. (Diat. d'Aur., p. 144).

Fragilaria construens var. circulare nor. - De forme circulaire ou en carré à angles largement arrondis, longueur 5 ì $8 \mu$; strics très délicates et difficilement risibles dans le baume.

Fragilaria elliptica Schum. (Diat. d'Anr., p. 145). - var. minor Grun. (Diat. d'Aur., p. 146).

Fragilaria minutissima Grun. -- Tout à fait semblable à la fig. 14, pl. $4 \bar{u}$ du Syn. de Tan-Heurck.

Fragilaria Zeilleri nov. sp. (Pl. X, fig. 9). -Valve bacillaire, à extrémités largement arrondies, plus rarement obtusément acuminées, longueur 30 ì $60 \mu$, largeur $\tau$ ì $10 \mu$; stries fines, mais distinctement granulées, au nombre de 10 i 11 en $10 \mu$, laissant au centre de la valre un pseudo-raphé bacillaire, occupant environ le tiers de la face valraire, à extrémités sulitement acuminées; les stries sont parallìles et très légèrement radiantes aux extrémités; face connectire rectangulaire, presque aussi large que la face valvaire, présentant à chaque bord une ligne marginale de granulations, au nombre de 10 à 11 en $10 \mu$, comme les stries, ce qui proure que ces granulations ne sont autre chose que les extrémités des stries de la face valvaire.

Tous dédions ce Fragilaria très distinct, au sarant paléobotaniste francais, MI. René Zeiller, Membre de l'Institut.

On troure beaucoup plus rarement une forme à valve plus acumince et ì stries plus distinctement granulées, dont les granules diminuent progressivement d'intensité, et ne s'effacent qu'au centre de la valve. - Tous 
avions pris tout d'abord cette forme pour un Nitzschia (Triblyonella), mais n'ayant pu noter de différence entre les deux bords de la valve, et par conséquent observer de carène, nous arons acquis la certitude que nous arions lì une variété remarquable du type, à laquelie nous proposons le nom de Fragilaria Zeilleri var. nitzschioides no:. (Pl. X, fig. 10).

Fragilaria Zeilleri forma anomala nov. (Pl. $\mathrm{X}$, fig. $10^{\text {bis }}$ ). - Il nous a paru intéressant de figurer ici une anomalie curieuse observée dans le dépôt; l'objet représente un groupe de frustules en rue connective, ayant subi une déformation bizarre au cours de son développement.

Fragilaria bidens Heib. (Consp. pl. 5, fig. 14). - - var. major Heib. (Diat. d'_ Luv., p. 143).

Fragilaria nitida nov. sp. (P]. XI, fig. 30). Valve bacillaire, très allongée, longueur 50 à $100 \mu$, largeur 5 à $7 \mu$, à extrémités coniques, arrondies, portarnt de chaque côté une petite pointe ou dent, analogue à celle que l'on observe aux extrémités de la face connec. tive des Thalanithria; ni stries, ni pseudo raphé visibles dans le baume; face connective exactement linéaire, très étroite, à extrénités tronquées; silice très épaisse. Espèce curieuse et bien distincte.

Fragilaria nitida var. delicatula not. (Pl. XII, fig. 29). - Silice beancoup plus délicate que celle du type; extrémités de la force connective dépourvues d'épines, et proportionnellement plus large; longueur 30 à $50 \mu$, largeur 3 à $4 \mu$.

Eunotia lunaris Grun. (Diut. d'Aur., p. 135). 
Eunotia polydentula Ehrb. var. fossilis nov. (Pl X, fig. 20). - Caratères généraux du tỵpe (Eunotia tridentula Ehrb.), en diffère par la présence de trois plis angulaires à la face ventrale, et en ce que la face dorsale porte des ondulations de forme différente, les trois médianes assez faibles sont encadrées de chaque côté par deux ondulations beaucoup plus prononcées.

Eunotia impressa Ehrb. Diat. foss. d'Aur., p. 27).

- - var. angusta Grun. (Diat.d'Aur., p. 134).

- monodon Ehrb. (Diat. d'Aur., p. 133).

- polyglypleis (irun. (Diat. d'Auv., p. 134).

- pectinatis Rab. (Diat. d'Auv., p. 132).

- Arcus Ehrb. (Diat. d'Aur., p. 130).

- gracilis Rab. (Diat. d'Auv., p. 131).

Epithemia Zebra Ktz. (Diat. d'Aur., p. 127).

- - rar. proboscidea (Diat d'Aur., p. 128).

- - var.minsı. F. H. (Diat. d'.lux., p. 129).

- turgida Ktz. (Diat. d'Aur., p. 124).

- - var.gremulata Gr. (Diat. d'Aur., p. 125)

- TestermamiiKtz. (Diat. d'Aur. p. 1:5).

- Hyndmannii TI. Sm. (Diat. d'Aur.,p.125)

\section{Epithemia Hyndmannii var. perlonga not.} -- On troùve, dans le dépôt de Joursac, une forme très semblable à l'Epithemia perlonga Pant., et qui se rattache certainement à l'Epithemia Hyndmam ï IV. Sm.; elle ne diffère de la Diatomée fossile de Hongrie (Pant., Lng. III, pl. 16, fig. 235) que par sa taille moins grande, et en ce que sa largeur décroit un peu du milieu vers les extrémités, tandis que l'Epithemia perlonga de Pantocseck a les burds parallèles. 
Rhopalodiagibberula Ehrrb. (V.-H.,Diat.,p.30, fig.825) $=$ Epithemia gilberula Ehrb. (Diat. d'Aux., p. 129.

- - var. producta Grun (1862, pl. 61, fig.9).

- gibla Ehrb. (T.-H., pl. 32, fig. 1 et 2).

- - var.paralleluGr.(V.-H.Synn.,pl.32,fig.3)

- - var.rentrirosa Gr.(T.-H.Synn.,pl.52, fig. 4-3)

Mantzschie amplioxys Gr. (Diat. d'Auv., p. 162).

- - var. intermedia Gr. (Diat.d'Aur.,p.162)

Nitzschia Brebissonii II. Sm. (Diut. d'Aur., p. 168).

- vitrea Norm. (That. d'Aux., 1. 170).

Grunouria Tabellaria V.-H. (Diat. d'.1uz., p. 166).

C'ymatopleura elliptica II. Sm. (Diat. d'Aur., p. 160). - var. sulbconstricta Gr. (Liat. d’Au:, p. 160)

Surivella gracilis Grun. (Thut. d'Aur., p. 180).

Surirella gracilis Grun. var. constricta no: (Pl. XI, fig. 31). - Longueur moyenne $85 \mu$, largeur maximum $17 \mu$, largeur minimum $12 \mu$; tout à fait semblable à l'espèce figurée par Tan-Heurck dans son Synopsis des Diutomées de Dielgique, pl. 76, fig. 16, mais au lieu d'aroir les flancs droits comme le trpe, notre variété présente une constriction très forte à la partie centrale.

Surirella tenera Greg. (Diat. d'. Iur., p. 180).

Surirella Pagesi nov. sp. (Pl. X, fig. 24). - De forme elliptique, à extrémités coniques et semblables; longueur $115 \mu$, largeur $38 \mu$; côtes au nombre de 2 en $10 \mu$, à tête très forte, mais diminuant rapidement d'intensité jusqu'au pseudo-raphé, qui est formé d'une ligne faible légèrement sinueuse. 
A. Schmidt représente ( $A t l$., pl. 23 , fig. 2 ) une forme analogue qu'il rattache an Surirella lifirons Ktz. mais cette dernière espèce est plus lenticulaire, ses extrémités sont plus pointues, et ses côtes beaucoup plus robustes.

Nous dédions cette belle Diatomée à M. Pagès-Allary, en reconnaissance des documents nomlireux que nous devons à son inépuisable a mabilité.

Surivella bifrons Ktz. (Diat. d':Aur., p. 177).

- robusta Ehrb. (Diat. "Aue., p. 180).

Campylodiscus costatus Bright. (Diat. d'Aur., p.182).

Opephora Martyi F. Hér. (Diat. foss. d' Aur., pl. 43).

Opephera Martyi var. capitata nor. (Pl. X, fig. 16). - Differe du type par sa taille et par son extrémité supérieure légèrement rétrécie et capitée.

Opephora cantalense nox.sp. (Pl. X, fig. 14). - De forme ovalaire plus ou moins allongée, quelquefois presque elliptique, surtout dans les petites formes; longueur 15 à $25 \mu$; côtes très robustes, an nombre de $5 \frac{1}{2}$ à 6 en $10 \mu$, parallèles, celles des extrémités toujours légèrement radiantes, laissant an centre de la valve, un pseudo-raphé large, fusiforme; face connective conique, parfois très fortement développée, surtout dans les petits exemplaires; côtes moins robustes que sur la face valvaire, terminées sur les bords par un granule, et plus courtes anx extrémités qu'au milieu.

Se distingue de l'Opephora Martyi par la forme de l'aréa, par le nombre et la diposition des côtes, ainsi que par la striation de la face connective. Très commun sur plusieurs points du dépôt.

Opephora cantalense var. capitata not. 
(Pl. X, fig. 15). - En général de taille plus grande, atteignant jusqu’à $45 \mu$, portant, à la partie supérieure, un étranglement surmonté d'une tête arrondie légèrement moins large que le corps de la ralve.

Tetracyclus stella E. (Diut. foss. d'. Lur., Pl. TIII, fig. 9)

Tetracyclus stellare nor. sp. (Pl. XI, fig. 23). - Affecte la forme presque régulière d'une étoile à huit branches; diamètre de 25 à $30 \mu$. Cette belle espèce est intermédiaire entre le Tetracyclus castellatum et le Tetracychus elegans; dont il nous paraît une forme dérivée.

Tetracyclus emarginatus E. (D. d'Aur., Pl. III, fig. 27). var.? - Nous arons observé un fragment d'un Tetracyclus qui pourrait être rapporté au Tetr. emarginatus, mais dont la forme reconstituće serait presque circulaire.

Tetracyclus Peragalli nov.sp. (Pl. X, fig. 21). - Espèce de petite taille; longueur de la valve 15 ì $20 \mu$, à extrémités rostrées, non capitées; côtes courbes, au nombre de 6 en $10 \mu$; stries et pseudo-raphé invisibles dans le baume.

Nous dédions ce Tetracyclus au savant diatomiste français, M. le Commandant Maurice Peragallo.

Tetracyclus Peragalli var. eximia not. (Pl. X, fig. 23). - Plus grand et de forme plus carrée que le type, longueur $3^{\prime} \mu$; côtes presque rectilignes et plus écartées, $3 \frac{1}{2}$ en $10 \mu$.

Tetracyclus Peragalli var. major nov. (Pl. X, fig. 22). - Encore plus grand que la variété eximia, longueur $37 \mu$; de forme générale semblable au type, mais à côtes écartées; toutefois le nombre des côtes in- 
complètes est assez grand, de sorte que, sur les bords de la valre, l'écartement des côtes est trìs roisin de celui observé dans le type.

Melosira undulata Ktz. (Diat. foss. d'Aur., p. 22).

- -var.producta A.Sch.(Ihiat.foss.rl'Aux.,p. 22)

- - forma hungarica A.S. (Atl.,pl.189,if.8-15)

Melosira undulata var. debilis nov. (Pl. X, fig. 27). - Se distingue du trpe par les parois du frustule non renfées et d'épaisseur uniforme; par les stries plus serrées et plus fines, au nombre de 15 en $10 \mu$ sur la face connective

Melosira Boulayiana Per. (Diat. foss. d'Aun., fig. 27-28).

Melosira imperfecta nor. sp. (Pl. X, fig. 31). - longueur du diamètre 68 à $72 \mu$; valre à parois très épaisses, surface lisse, à l'exception d'une couronne marginale formée de petites granulations décussées, très fines, au-dessous desquelles on aperçoit les stries irrégulières de la tranche de suture des valves.

A. Schmidt a dessiné (Att., pl. 176, fig. 23) une Diatomée fossile de Richmond, qui parait avoir heaucoup d'analogie avec celle de Joursac, mais la couronne marginale est irrégulièrement striée au lieu d'être formée par des points décussés.

Melosira minuta nov. $s p$. (Pl. X, fig. 32). Très petite espèce de 4 à $12 \mu$ de diamètre; vue connective rectangulaire ou carrée, à angles presque aigus; frustule moins long ou égal au diamètre de la valve; ainsi que le montre la vue connective, la valve est plate; à un grossissement faible, la surface valvaire paraît lisse, mais à un grossissement de 800 à 900 diamètres, 
on constate, surtout chez les grands exemplaires, qu'elle présente une granulation très légère formée de petites macules, et, de plus, deux ou trois granules fins, bien visibles; dans Jes petits exemplaires, on ne trouve plus que deux ou même un seul granule placé ordinairement tout à faitsur le bord de la valve, ce qui le rend peu visible.

Ne peut être assimilé ni au Melosira varians Ag., qui est plus grand et a les frustules plus longs que le diamètre, ni au Melosira distans Ehrb. var. nivalis WV. Sm., ni aux petites formes des Melosira cremulata Ktz. et granulata Ehrb., qui ont toujours des granulations très visibles, tant sur la face valvaire que sur la face connective.

Alelosira temuissima Grun. (Diat. d' Auv., p. 188).

- crenulata Ktz. (Diat. d’.tur., p. 186).

- - var. ambigua Grun. (Diat. d' Iur.,p. 187)

Melosira Camusi nor. sp. (Pl. X, fig. 29). Diamètre de 10 à $15 \mu$ de longueur, à surface lisse ou très légèrement granulée sur les bords dans lés grands exemplaires, et présentant toujours une couronne de granulation sur le bord de la valve; face connective plus longue que le dianètre, et proportionnellement d'autant plus longue que le diamètre est plus petit; sur la face connective, on compte 9 lignes longitudinales de granules en $10 \mu$; ces lignes sont quelquefois obliques ou en spirale; granules ronds, décussés ou non d'une ligne à l'autre, au nombre de 10 en $10 \mu$ sur la ligne.

Diffère du Melosira granulata Ehrb. par sa face valvaire non fortement granulée, et par sa face connective plus finement striée; se rapprocherait davantage du Melosira Carconensis Grun. (V.-H. Syn. pl. 87, fig. 27), 
mais celui-ci est plus finement strié, et surtout les granules sont beaucoup plus serrés et plus petits. - Très commun dans l'échantillon étudié sous le $\mathrm{n}^{\circ} 2$.

Ce Melosira est dédié au sarant botaniste parisien, II. E.-C. Camus.

Melosira Camusi var. conica $n x$. (Pl. X, fig. 30). - Se distingue nettement du type, par la forme tronconique du frustule en rue connectice. - Daus ses "Diatomées de Surtarlurant-Lac" (1900), Östrup représente une variété analogue du Melosira distans Ehrb, et qui nous parait encore plus conique.

Merosira granulata Ehrb. (Diat. d'Aur., p. 186).

Melosira granulata var. australiensis V.-II. - Très commun dans l'échantillon étudié sous le $\mathrm{n}^{\circ} 17$, et tout à fait conforme à la fig. $14, \mathrm{pl} .87 \mathrm{du}$ Synopsis de Van-Heurck.

('yclotella Iris F. Hérih. (Diat. foss. Pl. VIII, fig. 29). -. - var. integra (Diat. foss. d'. Lur. Pl. VIII, fig. 31).

Gyclotella perforata nov. sp. (Pl. X, fig. 35). -- T'etite espèce ì diamètre très variable, 5 à $18 \mu$; face valvaire striée sur environ les deux tiers du rayon; les stries, au nombre de 10 à 13 en $10 \mu$, suirant la grandeur du frustule, sont lisses, et portent, près du bord de la valve, un petit granule peu brillant; chez les petits exemplaires les stries sont toutes sensiblement égales, tandis que chez les plus grands, elles sont altemativement longues et courtes, et leurs extrémités viennent aboutir presque exactement ì deux cercles concentriques; la partie centrale de la valve, lisse dans les petits exemplaires, légèrement maculé dans les plus grands, 
porte, dans les uns comme dans les autres, un trou très apparent placé excentriquement; les trous des deux valves d'un mếme frustule sont diamétralement placés par rapport au centre; la surface striée de la valie est bombée, et le centre lisse présente une dépression excentrique, dont le point le plus las est occupé par le trou; silice robuste. - Espèce lien définie, et caractéristique du dépôt par son abondance.

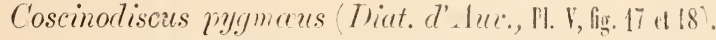

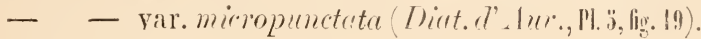

Coscinodiscus pygmæus rar. minutissima nox. - Toujours de très petite taille; diancitre 8 à $10 \mu$; commun dans l'échantillon étudié sous le no 14 .

Stepleanodiscus Astrea Ktz. (Diat. d' Lur., p. 193).

En totalisant les Diatomées mentionnées daus cette étude, on trouve qu'elles sont au nombre de 211, coniprenant 135 espèces et rariétés déjà conmues et 76 nouvelles pour la flore diatomique générale.

De tous les dépôts lacustres de l'Europe centrale nous n'en comnaissous pas qui aient livré une florule aussi variée.

$\Lambda$ propos d'un dépôt remanié, l'indication de la fréquence et de la rareté des espèces nous paraît dépcurrue d'intérêt; une Diatonée très commune sur un point étant sourent nulle ou très rare sur d'antres points, il en résulte que les notations CC et RiR peurent lui être attribuées indifféremment; nous arons dî, par consé- 
quent, nous borner à mentionner les espèces caractérss. tiques, c'est-à-dire celles que nous avons observées dans l'ensemble des échantillons étudiés, et en frustules ordinairement nombreux. Les espèces que nous considérons comme caractéristiques du dépôt de Joursac sont : Cocconeis Placentula et lineata; Fragilaria construens var. renter, Zeilleri et nitida; Eprithemia IIyndmannii: Opephora Martyi; Melosira undulata, minuta et crenulata; Coscinodiscus pygmceus.

En attendant la publication prochaine de la Flore miocène de Joursac, en collaboration avec notre savant compatriote, M. P. Marty, voici la florule phanérogamique que nous a livré le beau dépôt dont nous venons de faire connaître la florule diatomique.

\section{CHAMPIGNONS}

Depasea Feronice Ett. Rhintisma Planerce Ett.

Tylomites Aceris decipientis Ett.

\section{MUSCINÉES}

Muscites joursacensis nov. sp. I Hypnum Mastodontum N. Boul.

\section{PIIANÉROGAMES}

Juniperus dirupacea Lab.

- Var. pliocenica Rér.

Pinus sp., cf. P. Larix L.

Picea, cf. P. Mrorinda Link.

Abies Rumesi Sap.

Glyptostrobus europceus $\mathrm{Ir}$.

Pliragmites ceningiensis $\mathrm{Hr}$.

Typhas sp.

Betula elliptica Sap.

- prisca Ett.

- oxiyodonla Sap.

- alba L.

Almus cordifolic Ten.

- glutinosa Gartn.

- insignis X. Boul.

Carpinus pyramidalis $\mathrm{Hr}$. 
Curpinus orientatis Lamk.

- Belulus I.

- Grandis Ung.

Ostrya atlantidis Ung.

Corylus A cellana $\mathrm{L}$.

Fagus Feronice Ung.

- pliocenica Sip.

- siliatica L.

Quercus tenerrina $\mathrm{O}$. Web.

- Weberi Ung.

- Dirymeja Ung.

- mediterranea Ung.

- phillyreouiles A. Gray.

- Coccifera L.

- Buchii O. Web.

- Lucumonum Gand.

- pseulo-castanca Gœpp.

- Cardanii Mass.

- Etymodiys Mass.

- Senogallensis Mass.

Salix varians Gopp.

- alba L.

- cinerea L.

Populus Gandini F. O.

- Tiemula L.

Planera Ungori Ett..

C'7mus sp.
C'eltis Japreti Ung.

-- trachytica Ett.

Ficus Laurenti nor. sp.

Ficus sp.

Artocarpilium u'etteraricum Ett.

Coccoloba sp. (?)

Laurus mimigenia U’ng.

Sassaficas cantalense N. Boul.

Cypselites sp.

Cypselites sp.

Fraximus Ormus L.

Porana ceningiensis $\mathrm{Hr}$. (?)

Bumelia bohemica Ett.

Parrotia pristina Stur.

Acer tritobatum A. Br.

- decipiens A. Br.

- Tretuin C.-A. Mey.

-- - var. pliocenicum Sap.

Rhamnus, of. R. alpina L.

Juglans regia L.

Carya minor Sap.

Pterocarya denticulata $\mathrm{H}$.

Plelea Pagesi nov. sp.

Cerasus palcoavium nov. sp.

Cosalpinia sappanoides nor. sp.

Gleditschia allemanira IIr.

Robinia pseudo-acacia L.

II. Boule, d'après de Saporta et MI. l'abbé Boulay, avait fait connaître à Joursac 21 formes végétales distinctes, dont 15 déterminées spécifiquement et 6 déterminées génériquement.

Le nombre des espèces de la liste précédente est de 73 , réparties dans 25 familles et 45 genres.

11 résulte de cet appoint que, de toutes les flores locales, miocènes et pliocènes de la France, celle de Joursac est la plus riche.

Les éléments de cette florule seront décrits avec le 
plus grand soin, et figurés dans une quinzaine de plan. ches d'une exactitude irréprochable.

La distribution géographique de ces plantes, les rapports et les dissemblances de leur ensemble avec les flores du passé et avec la flore actuelle, seront aussi l'objet de recherches bascées sur des termes de comparaison bien établis.

Ces considérations d'ordre général, nous permettront de constater, par exemple, que deux espièces fossiles de Joursac habitent aujourd'hui l'Afrique; l'Europe en possède 21 ; l'Asie également 21 , et l'Amérique septen trionale 10 ; nous en conclurons que la flore tertiaire de Joursac est essentiellement européo-asiatique, avec un sixième environ d'espìces américaines; elle constitue actuellement une flore montagnarde qui, vers 700 on 800 mètres d'altitude peuple le systène alpin-himalaven tel qu'il a été défini par M. Suess.

Les éléments de la florule diatomique seront l'objet de recherches comparatives analogues. 


\section{DÉPOT D'ANDELAT}

Le dépôt d'Andelat est à 860 mètres d'altitude et à 4 kilomètres N.-O. de Saint-Flour, au lieu dit Trou de l'Enfer, en aval de la jolie cascade du Rozas, près du village d'Andelat.

D'après les documents très précis, communiqués par II. Lauby, la couche à Diatomées a une épaisseur de 12 à 15 centimètres, et se trouve intercalée entre deux assises de cinérite; son inclinaison du S. au N. est de $17^{\circ}$.

Nous devons les matériaux étudiés à l'amabilité de MIM. P. Narty et A. Lauby; la masse diatomifère est constituée par une argile micacée d'un gris noirâtre, très homogène, non feuilletée; l'examen microscopique ne rérèle aucune trace d'éléments andésitiques, par conséquent, sa formation est antérieure à l'émission de ces produits volcaniques.

Comme pour tous les dépôts remaniés ou fortement comprimés, les frustules sont en général très fragmentés, du moins chez les grandes espèces et les formes bacillaires; néanmoins les fragments étant très nets, ils se prêtent assez facilement à une détermination exacte, très rarement douteuse.

Les échantillons examinés nous ont livré les espèces suivantes:

Cocconeis I'lucentula Ehrb. (Diut. d'_Luv., p. 44).

- - var. minor F. Hérib. (Diat.d’Aue., p.218)

Navicula Pupula Ktz. var. major nor. (Pl. XII, fig. 7). - Diffère du type de Kützing par sa taille plus 
grande, longneur $56 \mu$; par sa forme plus bacillaire; par son aire hyaline centrale progressivement arrondie, au lieu d'être stauronéiforme; par les stries centrales plus écartées que les autres, et alternativement courtes et longues, les plus courtes étant environ d'une longueur moitié de celle des plus longues.

Navicula Gendrei F. Hérib. Pl IX, fig. 31).

- gastrum Donk. (Diat. d'Aur., p. 102).

Navicula Placentula Ehrb. var. major nov. (Pl. XII, fig. 6). - Présente les caractìres du Nav. Plucentula Ehrb., mais en diffère par sa grande taille, longueur $65 \mu$; par les stries centrales raccourcies, inégales, et entremêlées de stries beaucoup plus courtes et presque inarginales.

Naricula joursacensis F. Hérib. (Pl. IX, fig. 15).

-_ radiosa Ktz. (Diat. d'Aur., p 99).

- tenella Bréb. (Diat. d'Alur., p. 100).

- Esox Ehrb. (Diat. d'Aur., p. 82).

- BerriatiF.H. var. minor nor. Pl. IX,fig.25'.

Cymbella Laubyi F. Hérib, var. lanceolata nov. (Pl. XII, fig. 12). - Se différencie du type par sa forme plus élancée, par ses extrémités beau oup moius rostrées, et par les stries de la région ventrale plus serrées, au nombre de 12 en $10 \mu$; longueur de la valve $65 \mu$, largeur $18 \mu$.

Cymbella leptoceras Ktz. Diat. d'-Aux., p. 66).

Gomphonema intricatum Ktz. (Diat. d'Aur., p. 57).

- _ var. pumilaGr. (Diat.d'Auv.,p.57).

- Gilloti F. Hérib. (Pl. IX, fig. 7). 
Fragilaria Gustacei F. Hérib. (Pl. X, fig. 13).

- construens Grun. (Diat. d'Auv., p. 143).

- - var. venter Gr. (Diat. d'Aur, p. 144).

- Zeilleri F. Hérib. (Pl. X, fig. 9).

Epithemia turgida Ktz. (Diat. d'Aur., p. 124).

- HyndmannïT. Sm. (Diat.d'Auv., p.125).

- - var. perlonga nov. (dépôt de Joursac).

Rhopalotia gibierula Ehrb. (Diat. d'Auv., p. 129).

- - - var. producta Gr. (Diat. d'A Luv., p. 129).

Grunoura Tabellaria Grun. (Diat. d'Aur., p. 166).

Opephora Martyi F. Hérib. (Diat. foss. d'Aur., p. 43 ).

Opephora Martyi var. robusta nov. (Pl. XII, fig. 21). - Se distingue du type par sa silice beaucoup plus robuste, par ses stries plus fortes, quelquefois divisées en travers, division résultant de ce que la côte est formée de deux lignes parallèles de granules opposés, et souvent peu visibles; longueur très variable, de 30 à $60 \mu$, largenr, au contraire, peu variable, de 7 à $8 \mu$. On trouve, dans ce dépôt, trois formes principales : les petits exemplaires sont rhombiques, les grands exemplaires sont ovalaires ou lancéolés. -- La figure 21 représente l'une des plus grandes formes.

Nous avons dessiné, Pl. XII, fig. 2.2, une anomalie du type très curieuse par sa striation; cette déformation est l'analogue du Naricula viridis forma anomala (Diat. foss. d'Lux., Pl. VII, fig. t) trourée dans le dépôt quaternaire de Verneuges (Puy-de-Dôme).

Cyclotella perforata F. Hérib. et M. Per. (Pl. X, fig. 35).

Les Diatomées constatées le plus sourent dans le 
dépôt, et que l'on peut considérer comme espèces caractéristiques, sont : Fragilaria Zeilleri et Gustarei; Naricula Esox et Cyclotella perforata.

L'examen de la liste d'Andelat montre clairement que ce dépôt est tout à fait analogne à celui de Joursac, dont il est le prolongement; d'ailleurs, les deux masses diatomiques sont à la même altitude et occupent sensibement le même nivean stratigraphique; de plus, les quelques plantes fcssiles, signalées à Andelat par de Saporta, se trouvent à Joursac, telles que: Carpinus r.?. ramidalis Heer, C. orientalis Lamk, Acer triblatum A. Br., etc.; enfin, les éléments lithologique sont absolument identiques pour les deux dépôts. 


\section{DÉPOT DE CHAMBEUIL}

Le dépôt de Chamberil se troure à 2 kilomètres en a val du village de Laveiss:ère, entre Iurat et le Lioran, sur la rive droite de l'Allagnon, et à 950 mètres d'altitude, au cœur même du volcan du Cantal, c'est-ì-dire au point où devait s'ouvrir le cratère. Il est connu des géologues sous le nom d'argiles ligniteuses de Chambenil.

L’âge géologique de ces argiles a été très exactement déterminé par M. Boule. Le savant professeur a constaté qu'elles alternent arec des sédiments ligniteux et des tufs ponceux de nature trachytique; or, on sait que la trachyte est intercalé entre le basalte ancien et la formation andésitique, il en résulte que les argiles diatomifères sont contemporaines du trachyte; leur formation date done du Miocène.

Les argiles schisto-ligniteuses de Chambeuil contiennent peu de Diatomées; en outre, les frustules sont très fragmentés; à part les petits exemplaires discoüdes du Melosira temissima, les autres espèces ne sont représentées que par des fragments, dont la détermination spécifique est très difficile, et assez souvent d'une attribution douteuse.

De tous les dépôts diatomifères cantaliens, celui de Chambeuil est le seul qui nous ait présenté les caractères d'un sédiment nou remanié; de plus, nous avons acquis la conviction que ces argiles sont un lambean en place du rlépôt initial, d'où proviemnent toutes les argiles à Diatomées étudiées sous les noms de dépôts de 
Neussargues, Auxillac, Celles, Joursac, etc., etc. Ces masses à Diatomées ne sont pour nous que des lambeaux de projection (Auxillac, Celles, Neussargues), ou de restractification par les eaux (Joursac, Andelat), d'un raste dépôt initial de formation miocène.

Nous devons nous borner ici à l'indication de ce fait très important pour la diatomologie cantalienne; nous réservant de le discuter en détail dans la Flore miocène de Joursac.

Les échantillons étudiés nous ont été communiqués par MI. Marty; les Diatomées que nous y arons constatées sont:

Navicula major Ktz. (Diat. d'Auv., p. 82).

- oblonga Ktr. (Diat. d'Auv., p. 98).

- Legumen var. vix-undu'ata V.-H. - Conforme au dessin de Van-Heurck (Syn., pl. 6, fig. 17), mais plus grand; longueur $120 \mu$, largeur $22 \mu$; stries au nombre de 6 en $10 \mu$.

Stauroneis Phanicenteron Ehrb. (Diat. d'Aur., p. 75).

Fragilaria Irevistriata Grun. (Diat. d'Auv., p. 146). - - var. pusilla Gr. (Diat. d'Auv., p. 146). - construens Grun. (Diat. d'Aur., p. 143). - _ var. venter Grun. (Diat. d’Aue., p. 144).

Actinella pliocenica F. Hérib. var. tenuistriata nov. (Pl. XII, fig. 15). - Tariété très remarquable, bien distincte du type (Diat. foss. d'Aur., pl. S, fig. 7), par sa forme plus grêle; par sa striation plus serrée, stries au nombre de 12 en $10 \mu$, et par la grosse extrémité moins atténuée. La grande ahondance de cette Diatomée brésilienne, dans les argiles ligniteuses de Chambeuil, confirme la détermination du type trouré 
dans le dépôt de Celles, car nous arons pu observer des frustules complets, montrant clairement que la Diatomée appartient bien au genre Actinella.

Eunotia lunaris Grun. (Diat. d' Aur., p. 135). - pectinatis Rab. (Diat. d'Aur., p. 132).

- - var. stricta Rab. (Thiat. d'Aur., p. 132).

Melosira Camusi F. Hérib. ('']. IX, fig. 29). - canalifera F. Hérib. (Diat. d'Auv., p. 224). - tenuissima Grun. (Diat d'Auv., p. 188).

A l'exception de l'A. Ictinella pliocenica var. temuistriata et du Melosira tenuissima, les autres Diatomées ne sont représentées que par de rares exemplaires, plus ou moins fragmentés. 


\section{DÉPOT DE FRAISSE-BAS}

Fraisse-Bas est à côté de Chambeuil, ¿̀ la base du flanc gauche de la rallée de l'Allagnon; le dépôt de cette Iocalité n'est donc pas autre chose qu'un affleurement ou un prolongement de son voisin.

Grâce aux envois volumineux et variés, recus de notre aimable compatriote, MI. Pagès-Allary, nous avons ju établir une liste d'une vingtaine d'espèces, et compléter ainsi la florule sommaire de Chambeuil.

Nacicula major Ktz. (Diat. d'Aue., p. 80).

-. nobilis Ehrb. (Diat. d'Auv., p. 80).

- giliba Elrb. (Diat. d'Auv., p. 92). - La Navicule de Fraisse-Bas est plus petite que la forme type d'Ehrenberg; la longueur de la valve est à peine de $72 \mu$, et la striation est plus fine et un peu plus serrée.

Stauroneis Phenicenteron Ehrb. (Diat.d' Aur.,p. 75).

Ampleora libyca Ehrb. (Tiat. d'Aux., p. 63).

Complionema intricatum Ktz. (Tiat. d'Aur., p. 57). subclaratum Gr. (I)iat. d'Aur., p. 55).

Synedra LTha var. danica Ktz. (Thiut. d'_Lue., p. 138).

Fragilariu Zeilleri F. Hérib. (PI. IX, fig. 9).

Actinella pliocenica var. temistriata nov. (PI. III, fig. 15a).

Actinella pliocenica var. serpentina nov. 
- Variation très curieuse par la forme ondulée de sa valve, qui lui donne l'aspect d'un petit serpent; stries au nombre de 12 en $10 \mu$, comme dans la variété précédente.

Eunotic gracilis Rab. (Diat. d'Aur., p. 131).

- monodon Ehrb. (Thiat. d'Alux., p. 133).

- parallela Ehrb. (Diat. d'.lue., p. 133).

- pectinalis Rab. (Diat. d'Amr., p. 132).

Cymatopleura sp. (?) - Un fragment trop incomplet pour se prîter à une détermination spécifique.

Surirella sp. (?) - Même observation.

Opephora Martyi F. Hérib. (Niat. foss. d'Aur., p. 43).

Tetracyclus emarginatus WV. Sm. (Diat. d'.1ur., p. 158)

Coscinodiscus rlispar F. Iérib. et M. P. (Dict. d'Aur., p. 194). - Espèce saumâtre caractéristique du dépôt de Varennes (Puy-de-Dôme); sa constatation à FraisseBas, où elle est commune sur plusieurs points, est un fait intéressant.

Cette Diatomée se trouve aussi ì Faufouilhoux, sous une forme un peu différente.

Parmi les Diatomées très répandues dans les matériaux étudiés, nous mentionnerons : Fragilaria Zeilleri, Actinella pliocenica var. tenuistriata et Coscinorliscus dispar.

D'après les deux listes de Chambeuil et de FraisseBas, on yoit que toutes les Diatomées de ces deux lambeaux en place ont été déjà signalées soit à Joursac, soit ì Celles.

Nous n'avons pas trouvé de feuilles d'arbres dans les 
matériaux reçus de M. Pagès, mais nous arons constaté de nombreux débris de Glyptostrobus europceus Heer, tr̀s petite Conifère indiquée aussi à Joursac, et dont l'espèce analogue, Glyptostrobus heteroplyyllus Endl., vit anjourd'hui à demi submergée dans les rizières de la Chine; nous avons encore observé des fragments de feuilles de Potamogeton, que l'on peut rapporter à Potamogeton quinqueneris $\mathbf{N}$. Boul.

Ces deux plantes aquatiques deraient se développer à profusion sur les bords spongieux du lac miocène, oì elles ont été enfouies par les sédiments argileux charriés par les eaux courantes; d'ailleurs, par leur nature, elles sont aptes, comme les Mousses, à résister, sans se décomposer, à un milieu humide, contrairement aux feuilles d'arbres, dont la décomposition ne tarderait à se produire.

Ainsi, nous arons dans le Glyptostrolus europecus et le Potamogeton quinquenervis de Chambeuil-Fraisse-Bas, un exemple très remarquable de deux plantes fossiles trouvées dans un dépôt diatomifère en place; c'est lì un fait absolument exceptionnel, que nous n'arons jamais constaté dans les dépôts quaternaires du Puy-de-Dôme. 


\section{DÉPOT DE FAUFOUILHOUX}

Le dépôt de Faufouilhoux, près de Murat, se rattache à celui d'Auxillac; l'un et l'autre sont deux lambeaux de projection, non remaniés par les eaux, du dépôt initial de Chambeuil-Fraisse-Bas.

C'est encore à l'obligeance de M. Pagès-Allary, que nous sommes redevable de la totalité des matériaux étudiés.

La liste suivante a été dressée d'après l'examen d'une dizaine d'échantillons, plus ou moins différenciés par leurs caractères physiques, couleur, densité, consistance, etc., et provenant de divers points de la masse.

Rhoicosphenia currata Grun. (Diat. d'Auv., p. 51).

Achnanthes lanceolata Grun. (Diat. d'Auv., p. 49).

Navicula licapitata Lag. (Diat. d’Auv., p. 93).

- gigas Ehrb. (Diat. d’Auv., p. 81).

- major Ktz. (Diat. d'.Lue, p. 82).

- Reinhardtii Grun. (Diat. d'Aux., p. 102).

- rentricosa Donk. (I)iat. d'Auv., p. 113).

Stauroncis Phonicenteron Ehrb. (Diat.d'Auv., p. 75)

Pleurosigma attenuatum Ktz. (Diat. d'Aluv., p. 122).

Amphora libyca Ehrb. (Diat. d'Auv., p. (22).

Cymbella Bouleana F. Hérib. (Diat. d'Aur., p. 220).

- aspera Ehrb. (Diat. d'Aur., p. 69).

- cistula Hempr. (Diat. d' Jur., p. 70). 
Cymbella capitata $n o x$. sp. (Pl. IX, fig. 6). De forme elliptique, à extrémités tronquées et rostrées capitées; longueur de la valve 50 à $70 \mu$, largeur 15 ì $20 \mu$; raphé légèrement arqué, ì nodules terminaux ronds, petits, mais bien visibles et placés ì l'extrémité de la valve; nodules centraux légèrement tournés vers le bord ventral; aire hyaline axiale notable, lancéolée, très fortement dilatée autour du nodule médian, surtout du côté ventral; stries fines mais très visibles, non distinctement granulées, radiantes, celles de la face dorsale légèrement courbes, beaucoup plus serrées aux extrémités qu'au milieu de la valve, où elles sont au nombre de 6 en $10 \mu$ à la partie dorsale et de $7 \frac{1}{2}$ en $10 \mu$ à la partie ventrale. - Cette espèce, très distincte, se trouve aussi dans le dépôt de Moissac.

Cymbella conifera F. Hérib. (Diat. d'Aue, p. 220).

- cymbiformis Ehrb. (Diat. d'Auv., p. 69).

- lanceolata Ehrb. (Diat. d'Aur., p. 68).

- leptoceras Kitz.f. curta (V.H. Syn.,p.3, lig. 21)

- Pauli M.Per. F. Hérib. (Diat. d'Aur.,p. 70)

Gomplonema cantalicum F. Hér. (Diat. d'Aur., p. 219) - capitatum Ehrb. (Diat. d'Auv., p. 53). - oliraceum Ehrb. (Diat. d'. Iur., p. 61). - subclavatum Grun. (Diat. d'Aur., p. 55).

Synedra capitata Ehrb. (Diut. d'Aur., p. 139).

Fragilaria brevistriata Grun. (Diat. d'Alur., p. 146).

Eunotia gracilis Rab. (Diat. d'Auv., p. 131).

- polyglypleis Grun. (Diat. d'Aur., p. 134).

Epithemia turgida Ktz. (Diat. d'Aur., 1. 124).

Epithemia turgida Kitz. var. porcellus not. (Pl. X, fig. 19). - Cette forme a quelque analogie avec 
notre Epithemia turgida var. crassa du dépôt quaternaire de Ceyssat (Diat. d'Auv., Pl. III, fig. 16), mais elle s'en distingue par son aspect plus trapu, par ses extrémités non prolongées ni capitées, diminuant progressivement de largeur pour se terminer par une pointe relativement fine, arrondie et récurvée vers la face dorsale, présentant la physionomis d'un groin de porc.

Nous avons aussi observé cette variété dans des préparations du dépôt d'Auxillac.

Epithemia Hyndmannï W. Sm. (D. d'Auv., p. 125).

- Sorex Ktz. (Diat. d'Auv., p. 126).

- Zebra Ktz. (Diat. d'Auv., p. 127).

- - var. proboscilea Gr.(Diat.d'Aur.,p.123)

Rhopalodia gilba Ehrb. (Diat. d'Aur., p. 126).

Ilantischiin amphioxys Grun. (Diat.d'Aur, p. 162).

Cymatopleura Solea Bréb. (Diat. d'Auv., p. 161).

Surirella orata Ktz. (Diat. d'Auv., p. 175).

- saxonica Auersw. (Diat. d'Auv., p. 176.)

Campylodiscus costatus II.Sm. (Diat. d'Aur.,p.182)

Tetracyclus Boryanus (Pant.) var. minor nov. (Pl. XII, fig. 9). - Se distingue du type (Pant. Ung. Il, pl. 23, fig. 341) par sa taille beaucoup plus petite, longueur 25 i $35 \mu$; par ses côtes plus serrées, $31 / 2$ à 4 en $10 \mu$, interrompues au milieu de la valye par un pseudo-raphé étroit mais assez visible, et entre lesquelles on peut aperceroir de fines stries parallèles aux côtes.. - Le Salacia Boryana de Pantocseck = Castracania Boryana de Toni, a $70 \mu$ de longueur et deux côtes en $10 \mu$. Le Tctracyclus de Faufouilhoux diffère 
du Tetr. thomlus Ralfs. par la présence de stries intercostales.

Tetracychus ellipticus F. Hérib. (Diat. d'Auv.,p.159).

- Lamina Ehrb. (Diat. d'Aur., p. 159).

- emarginatus W.Sm. (Diat.d'Auv.,p. 158)

Melosira canalifera F. Hérib. (Diat. d’Aux., p. 224).

Melosira canalifera var. anastomosans nov. (Pl. X, fig. 34). - Diffère du type par sa face val. vaire plus granulée, à granulation plus fine, et dont les canaux, au lieu d'être seulement marginaux, s'étendent ordinairement jusqu'au milieu du rayon en se croisant; la granulation couvre parfois toute la valve, et les gra. nules sont d'autant plus petits qu'ils sont plus rapprochés du centre; la face connective présente quelquefois des canaux analogues à ceux de la face valvaire.

Melosira granulata Ehrb. (Diat. d'Aur., p. 186).

- tenuis Grun. (Diat. d'Aur., p. 188).

- varennarum F.H.M.P. (Diat.d'Aui.,p.189)

Cyclotella Tris F. Hérib. (Diat. d'Aur., p. 189).

- - var. integra F. H. M.P. (Diat.foss. d'Auc.,p. 17).

- - var. ovalis F. H, (Diat. d'Auv., p. 225).

- - var. cocconeiformis F.H. (Diat.d'Auv., p. 225).

Stephanodiscus Astrca Ktz. (Diat. d'Aur., p. 193).

Coscinodiscus dispar var. radiata F. H. forma inermis nov. - Diffère du Coscinodiscus dispar var. radiata (Diat. d'Auv., Pl. V, fig. 23 et 24) par l'absence des épines marginales, par la striation moins serrée, par l'absence fréquente de granules au centre de la valve distinctement rayonnée, et que nous avons appelée valve inféricure. (Diat. d'Aur., Pl. V, fig. 23). 
Dans deux échantillons examinés, nous arons observé plusieurs frustules et même des filaments, formés par la réunion du Melosira granulata Ehrb. et de notre Me. losira canalifera, ce qui proure que ces deux Melosira appartiennent au même type spécifique. Il nous a paru intéressant de représenter ce fait curieux et instructif (Pl. XII, fig. 25).

Les espèces les plus répandues dans la masse du dépôt sont: Achinanthes lanceolata, Gomphonema cantalicum, Cymbella Bouleana et Puuli; Melosira granuluta, canalifera et rarennarum; Cyclotella Iris et ses variétés; Coscinodiscus dispar et ses variations.

Parmi ces Diatomées caractéristiques de Faufouilhoux, il est très remarquable de constater que Melosira varennarum et Coscinodiscus dispar sont en même temps caractéristiques du dépôt tertiaire de Varennes (Puy-de-Dôme). 


\section{DÉPOT DE SAINTE-ANASTASIE}

Sainte-Anastasie est à 5 kilomètres N.-O. de la gare de Neussargues, sur la ronte d'Allanche; le dépôt, récemment découvert par II. Pagès, est à proximité de la localité.

L'examen d'une dizaine de préparations nous a donné le résultat suirant:

Rhoicosphenia cureata Grun. (Diat. d'Aue., p. 51).

Cocconeis lincata Grun. (Diat. d'. Lur., p. 4t).

$$
\text { - - var.euylyptaGrun. (Diat.d'Aur., p.46). }
$$

Navicula Placentula Ehrb. (Diat. d'Aur., p. 102).

- Reinhardtii Grun. (Diat. d'Aue, p. 102).

-- radiosa Kitz. (Diut. d'.1ur., p. 99).

- lata Bréb.var.minor E. H.(Diat.d'.Luv., p. 85)

- borealis Ktz. (Diat. d'Aur., p. 86).

- rentricosa Donk. (V.H. Syn.,pl.12, fig. 24).

- major Ktz. (Diat. d'Auv., p. 8.2).

Cymbella aspera Ehrb. (Diat. d'Aur., p. 69).

- cistula Hempr. (Diat. d'Aur., p. 70).

- conifera F. Ilérib. (Diat. d'Aue, p. 220).

- cymbiformis Ehrb. (Diat. d'Auc., p. 69).

- lanceolata Ehrb. (I)iat. d'. Luc., p. 68).

- leptoceras Ktz. (Diat. d'Aut., p. 66).

Encyonema coespitosum Ktz. (Diat. d'Auv, p. 73).

Gomplionema cantalicum F. Hér. (Diat.d'Auc.,p. 219) 
Fragilaria lrevistriata Grun. (Diat.d'Aur., p. 146).

- capucina Desm. (Diat.d'Auv., p. 142).

- lapponica Grun. (Diat. foss. d'Aur., p. 21).

Eunotia polyglyphis Grun. (Diat. d'Aur., p. 134).

Epithemia Hijndmannï W.Sm. (Diat. d'Aur.,p.125)

- Sorex Ktz. (Diat. d'Auv., p. 125).

- turgida Kitz. (Diat. d'Aur., p. 124).

- Zebraki. var. minor F.II. (Liat.d'Aur.,p. 129).

Rhopalodia gilba Ehrb. (Diat. d'Aux., p. 126).

- - var. ventricosaGr. (Diat.d'Auv.,p.126).

ILantzsstia amphioxys Grun. (Diat. d'Aur., p. 162).

Nitzsclia fontico?a Grun. (Diat. d'Auv., p. 171).

Surirella gracilis Grun. var. eximia nov. Ressemble à notre Surirella gracilis var. constricta (Pl. XI, fig. 31), mais en diffère par sa taille beaucoup plus grande, longueur $150 \mu$; par sa constriction médiane plus forte, largeur au milieu $24 \mu$, au renflement $40 \mu$, et par ses extrémités plus acuminées; les côtes sont au nombre de $6{ }^{1 / 2}$ en $10 \mu$.

Surirella saxonica Auersw. (Diat. d'Auv., p. 176).

- tenera Greg. (Diat. d'Auv., p. 180).

Tetracyclus emarginatus IV. Sm. (Diat. d'Auv.,p. 158).

- rhombus Ralis. (Diat.d'Aur.,p. 160).

Melosira canalifera F. Hérib. (Diat. d'Aur., p. 224).

- Iramulata Elirb. (Diat. d'Auv., p. 186).

- tenuis Grun. (Diat. d'Auv., p. 188).

- varennarum I. P.F.H. (Diat.d’Aur.,p. 189).

Cyclotella Iris F. Hérib, et Br. (Diat. d'Aur.,p. 224).

- _ var.cocconeiformisf.H. (Diat.d'Auv.,p.22\%)

- - var. oralis F. H. (Diat. d'Auv., p. 225). 
Les espèces caractéristiques sont: Melosira tenuis, cxnalifera et granulata; Cyclotella Iris et ses variétés. Ces trois Diatomées, par leur abondance, forment au moins les deux tiers du volume des échantillons étudiés. Sainte-Anastasie est tout à fait semblable à Auxillac, et il est de toute évidence qu'il doit être considéré comme un lambeau de projection provenant du dépôt initial de Chambeuil-Fraisse-Bas. 


\section{DÉPOT DE MGISSAC}

C'est encore à II. Pagès-Allary que nous derons la découverte de ce dépôt, avec la plus grande partie des matériaux utilisés.

Le gisement est situé à proximité du rocher basalıique de Laval, à trois kilomètres N.-O. de la gare de Neus. sargues, au-dessus de la route d'Allanche, sur le flanc gauche de la vallée.

Nous en arons examiné deux affleurements, distants, en altitude, de 25 mìtres environ; l'affleurement inférieur, découvert en compagnie de M. Pagès (septembre 1902 ), se montre sur le talus d'un chemin rocailleux, ì quelques mètres au-dessus de la route.

Les échantillons étudiés ne provenant que de deux points, nous ignorons l'étendue du dépôt. La masse, de consistance ferme et de couleur jaunâtre, présente de nombreuses fissures tapissées d'une substance cireuse jaune, très analogue à l'ozokérite ou cire minérale. 11. Pagès a constaté aussi la présence de cette substance sur plusieurs points du dépôt de Celles, et en assez grande abondance.

Moissac, comme Sainte-Anastasie, Auxillac, Celles, etc., est un lambeau de projection arraché du dépôt initial de Chambeuil-Fraisse-Bas.

La liste suivante a été établie d'après l'examen de matériaux yariés et une soixantaine de préparations :

Achnanthes Flahaulti nor. sp. (Pl. X, fig. 17 et 18). - De forme lenticulaire, à extrémités prolongées 
et arrondies, portant à un des côtés de la valre une cloison en forme de fer à cheral; longueur de la valve 30 à $40 \mu$, largeur 10 à $18 \mu$; valve supérieure à pseudo-raphé étroit, lancéolé, se dilatant en pseudo-stauros au centre de la valve, d'un côté jusqu'ì la cloison, dont la surface est également lisse; de l'autre côté jusqu'à la moitié de la distance du centre au lord de la ralve; stries fines mais très nettes, non distinctement granulées, rayonnantes et légèrement courbes, la concavité tournée vers les extrémités, au nombre de 14 en $10 \mu$; du côté de la cloison les stries manquent devant le nodule médian, et sont interrompues sur la surface de la cloison qui est lisse; de l'autre côté les deux stries médianes sont écourtées; valve inférieure à raphé très délicat et à nodules très petits ; aire hyaline axiale très petite, se dilatant des deux côtés du nodule médian en un pseudo-stauros pointu; stries semblables à celles de la valve supérieure et ayant le même écartement, très rayonnantes, courbes, mais ell sens contraire de celles de la valve supérieure, cette courbure est obtenue par l'augmentation progressive de la longueur des stries du milieu de la valve qui aboutissent au pseudo-stauros, la strie médiane est très courte, les suivantes sont de plus en plus longues, chaque strie enveloppant la précédente, de sorte que la strie la plus longue, dont la poiute arrive près du nodule médian, a son pied très loin du milieu de la valre, et, par suite, elle est tıès oblique et très arquée.

Nous dédions cette espèce, bien distincte, à II. Ch. Flahault, le savant Directeur de l'Institut de Botanique de Montpellier.

Achenanthes lanceolata Grun. (Diat. d'Aur., p. 49).

- - var. elliptica Cl. (Diat.d'Auv., p. 219).

- lungarica Grun. (Diat. d’Aur., p. 48). 
Cocconeis Bonnieri nor. sp. (Pl. XI, fig. 24 et 25). - De forme largement ovale; longtieur $45 \mu$, largeur $25 \mu$; stries rayonnantes, composées de fins grauules allongés; valve supćrieure à pseudo-raphé très visible et s'arrêtant assez loin des extrémités de la valve; stries rayonnantes, courbes, au nombre de 11 à 12 en $10 \mu$ au milieu de la valve, plus écartées aux extrémités; valve inférieure présentant un anneau intramarginal semblable ì celui des Cocconeis Placentula et lineata, mais sur lequel les stries ne sont pas interrompues; aires lyyalines axiale et centrale nulles; raphé très délicat, stries rayonnantes, courbes, au nombre de 14 ì 15 en $10 \mu$.

Ce Cocconeis, très remarquable, est dédié à M. Gaston Bonnier, Membre de l'Institut.

Cocconeis lineata Grun. (Diat. d'Aur., p. 44). - - var. euglypta Grun. (Diat. d'Aue,p.46)

Navicula gibbula Clève (Syn. 1894, p. 140). Bien conforme à la description de l'auteur.

Navicula gibbula Cl. var. cantalica noe. (Pl. XI. fig. 15). - Diffère de la forme type de Clère par sa taille plus grande, longueur 60 à $70 \mu$, par son aire hyaline axiale très notable, fusiforme, arrondie autour du nodule médian; stries rayonnantes, fines, les médianes radiantes et écourtées, les suivantes parallèles entre elles, et par conséquent radiantes jusqu'aux extrémités de la valye, au nombre de 20 à 21 en $10 \mu$, excepté pour les centrales qui sont plus écartées et plus fortes.

Tacicula Bacillum Elırb. (Diut. d’Aur., p. 117).

Navicula ventricosa Donk, var. cuneata 
nor. (Pl. IX, fig. 29). - Se distingue du type (V. H. Syn. pl. 12, fig. 24), par ses extrémités cunceiformes, par ses nodules terminaux très petits, placés tout à fait anx extrémités de la valve, par son aire hyaline axiale plus large, dilatée en un stauros plus large, et par ses stries médianes parallèles au lieu d'être rayonnantes.

Naricula priducta IV. Sno. (Diat. d'Auv., p. 115).

Navicula Iridis Ehrb. var. subproducta nor. - Forme intermédiaire entre le Taricula Iridis Ehrb. et le Tacicula Columnaris Ehrb., c'est-à-dire entre les figures 1 et 3 de la pl. 49 de l'Atlas de A. Schmidt; ressemblant à la fig. 2 de la même planche, mais avec les extrémités atténuées.

Naricula ampleigomplues Ehrb. (Diat. d'Aur., p. 113) - cuspidata Ktz. forma craticula (D. Itur., pl II, fig. I"̈) - - var. Meribaudi M. P. (D.d'Aue, PI. IV, fig. 16) - spherophora Ktz. (Diat. d'Aux., p. 109).

- Gendrei F. Hérib. (Pl. IX, fig. 31).

Navicula mutica Ktz. var. producta V. H. - Bien conforme à la fig. 20, pl. 10, du Synopsis de Van-Heurck.

Savicula rostellata Ktz. (V. II. Syn. pl. 7, fig. 23). - gastrum Donk. (Diat. d'Aur., p. 102).

Navicula Chaberti nor. sp. (Pl. IX, fig. 18). De forme lenticulaire, à extrémités rostrées et arrondies; longueur de la valve $55 \mu$, largeur $20 \mu$; raphé fin, ì nodules terminaux ronds, petits, placés tout à fait à l'extrémité de la valve; aire hyaline axiale notable, lancéolée, arrondie autonr du nodule médian; stries fines mais très nettes, non distinctement granulées, d'autant 
plus radiantes et plus serrées qu'elles s'éloignent davantage du milieu de la valve, légèrement courbes, non alternativement longues et courtes an centre, où elles ont l'écartement de $7^{1} / 2$ en $10 \mu$.

Se distingue du Taricula I'lucentuia Ehrb. par son aspect plus délicat, par ses stries fines et non granulées, et par la disposition des stries centrales. Diftìre de notre Naricula Gomontiana par la forme de son raphé, par les stries plus serrées et plus délicates; dans le Navicula Gomontiana, elles sont plus robustes et de force décroissante du bord de la valve au raphé.

Cette Navicule est dédiée à M. le 10 Chabert, botaniste à Chambéry, médecin major en retraite.

Naricula Placentula Ehrb. (Diat. l'Auv., p. 102).

Navicula acephala nor. sp. (Pl. XI, fig. 18). - Petite espèce de $35 \mu$ de longueur, en forme de ménisque biconcave, ì extrémités arrondies et très légèrement rostrées; stries rayonnantes, finement granulées, au nombre de 10 en $10 \mu$, les centrales plus écourtées, presque marginales, laissant autour du nodule médian une aire hyaline stauronéiforme grande et carrée; aire hyaline axiale nulle.

Diftère du Naricula dicepluala II. Sm., dont elle a tout ¿̀ fait la striation, par ses extrémités ì peine un peu rostrées et non capitées.

Nacicula digito-radiata Greg'. (D. foss. d'Aur., p. 25)

- radiosa Ktz. (Diat. d’Auv., p. 99).

- - var. acuta Grun. (Diat. d'Aur.,p. 100).

- tenella Bréb. (Diat. d'Aur., p. 100),

- nolitis Ehrb. (Diat. d'Aux., p. 80).

- major Ktz. (Diut. d'Auv., p. 82).

- Esox Ehrb. (Diut. d'Auc, p. 82).

- decurrens E. var. curtecostata not. (II. II, lig. 27) 
Navicula Huei nor. sp. (Pl. IX, fig. 26). - De forme bacillaire à extrémités semi-elliptiques; longueur 50 à $60 \mu$, largeur 8 ì $10 \mu$; aire hyaline axiale notable, stauronéiforme au nodule médian; stries non distinctement granulées, au nombre de 10 en $10 \mu$, manquant des deux côtés au milieu de la valve, parallèles sur environ la moitié de la longueur de la valve, puis convergentes.

Se distingue du Naricula Spetzberyensis Clère (Clève S.yn. 1895, p. 82) par ses extrémités semi-elliptiques plutôt qu'arrondies, par ses stries moins serrées, les terminales convergentes; elles sont au nombre de 16 à 17 en $10 \mu$ dans l'espèce de Clève.

Nous dédions cette petite Navicule, très distincte, au savant lichénologue français, M. l'abbé Hue.

Navicula microstauron (Ehrb.) $=$ Stauroptera microstaura Ehrb. = Pinnularia microstauron Clève Cl. Sym. 1895, p. 7т). - C'est ì cette espèce que Clève rapporte, mais à tort, notre Nacicula divergens var. prolongata (Diat. d'Aur., Pl. IV, fig. 1).

Navicula divergens $\mathbb{T}^{*}$. Sm. var. undulatocuneata nov. (Pl. XI, fig. 12). - De forme bacillaire, légèrement triondulée, à extrémités coniques atténuées, largement arrondies; longueur 90 ̀े $100 \mu$, largeur $15 \mu$; stries assez courtes, au nombre de $\delta$ en $10 \mu$, manquant au milieu, radiantes vers le nodule médian, et fortement convergentes aux extrémités; aire hyaline axiale large, conique aux extrémités, en-uite linéaire et élargie autour du nodule médian où elle devient stauronéiforme.

Cette variété est intermédiaire, comme forme et comme striation, entre les figures 8 et 10 de la pl. $4 t$ de l'Atlas de Schmidt, non dénommées par cet auteur; 
la seconde est nommée, par Clève, Pimnlaria dieryens var. cuneata

Naricula mesoleptu Ehrb. (Diat. d'. Luv., p. 96). - - var. stuuroneiformis Gr. (I).d'Auc.,p.96)

Navicula decrescens nor. sp. (Pl. XI, fig.19). - Valve à bords triondulés, l'ondulation centrale étant la plus forte, extrémités rostrées, non capitées et arrondies; longueur $50 \mu$, largeur $8 \mu$; stries an nombre de! en $10 \mu$, très courtes et radiantes au centre, où elles sont tout à fait marginales, s'allongeant progressivement et se redressant jnsqu'aux extrémités, où elles touchent le raphé, sont convergentes et entourent le nodule terminal, en le laissant au milieu d'une aréa circulaire.

Assez semblable ì la figure 62 de la pl. 45 de l'Atlus de Schmidt, mais s'en distingue par le nombre de ses stries et la forme de l'aréa.

Diffère des Naricula gracillima, mesotyla ot mesolepta qui ont des contours analogues, par le nombre des stries et la forme de l'aréa.

Nacicula Termes Ehrb. (Diat. d'Auv., p. 97).

- - var. stauroneiformis V.H. (D.d'.Lur.,p.97)

Navicula bicapitata Lag. var. sulcata nor: (Pl. XI, fig. 10). - A beaucoup d'analogie avec Naricula bicapituta var. liybrida Grun. (V. H. pl. 6, fig. 9), mais notre Dia tomée en diffère nettement par la présence de deux lignes brillantes (côtes ou sillons) parallèles au raphé.

Navicula Braunii Grun. var. moissacensis nor. (Pl. XI, fig. 17). - Longueur 55 à 80 $\mu$, largeur 10 à $11 \mu$; stries au nombre de 10 en $10 \mu$. Diffère du type 
par sa taille plus grande, par ses stries plus écartées et plus courtes, et par son aréa stauronéiforme beaucoup plus large.

Naricula acrosplueria Bréb. (Diat. d'Aur., p. 93).

-- - var. levis F. H. etM.P.(D.d'Auv.,p.93)

Navicula acros phæria Bréb. var. bacillaris nou. (Pl. XI, fig. 13). - Valve presque exactement bacillaire, à extrémités arrondies, longueur 80 à $100 \mu$, largeur 11 ì $13 \mu$; stries presque marginales, très légèrement rayomnantes, au nombre de $9{ }^{1}{ }_{2}$ en $10 \mu$; aréa large et lisse, presque bacillaire; nodules tèrminaux tout à fait caractéristiques du Naricula acrosplaceria Bréb.

A. Schmidt figure ( $1 t$ t. pl. 43, fig. 17), sans la nommer, une forme très analogue, mais notablement turgide au milieu.

Navicula acrosphæria Bréb. var. elongata nov. (Pl. XI, fig. 14). - Valve très longue, $160 \mu$, et relativement étroite, $15 \mu$, presque linéaire, centre et extrémités à peine un peu renflés; stries au nombre de 9 en $10 \mu$, courtes, laissant au milieu de la valve une aire hyaline axiale large et lisse,

Diffère du Nacicula gibla Ehrb. par sa forme, son aréa large et brusquement diminuée aux extrémités, et du Taricula acrospluceria Bréb. par la forme de ses extrémités et de son aréa, qui est nulle près des nodules terminaux.

Naricula hemiptera Kitz. (Diat. d'Aur., p. 84).

Navicula hemiptera Ktz. var. angusta nor. (P]. XI, fig. 20). - - Plus allongé et plus étroitement elliptique que le type; longueur $70 \mu$, largeur $9 \mu$; stries 
au nombre de 8 en $10 \mu$, radiantes au centre, convergentes aux extrémités de la valve et interrompues au milieu.

Naricula hemiptera Ktz. var. Bielansiï F. Ilérib. (Diat. d'. lux., Pl. IV, fig. 10).

Navicula hemiptera Ktz. var. gibba nor. (Pl. XI, fig. 11). -- Talve hacillaire, centre et extrémités largement arrondis; longueur 120 à $130 \mu$, largeur 15 à $20 \mu$; côtes au nombre de 7 en $10 \mu$, courtes, presque marginales et radiantes au milieu, ò̀ elles manquent d'un côté sur une petite longueur, puis plus longues et droites, enfin convergentes et allongées jusqu'au raphé aux extrémités, laissant au milieu de la valve une aréa large, dilatée, stauronciforme d'un côté autour du nodule médian, et se retrécis ant subitement aux extrémités.

Navicula hybrita F. H. et M. Г'. (Diat. d'Auv., p. 85).

-- amplibola Clève (Cl. Syn. 1895, p. 45).

- - var.stauroneiformis nov. (D.d'Aux.,p.37)

- Berriati F. Hérib. (Pl. IX, fig. 21).

- - var. minor nov. (Pl. IX, fig. 25).

Stauroneis parvula Grun. (Clève, Synn.1894, p. 149). - Clève attribue à cette espèce les caractères suivants: «Valve linéaire lancéolée, avec des extrémités obtuses ou légèrement rostrées; longueur de la valre 20 ì $25 \mu$, largeur $5 \mu$; stauros large, transversal; stries radiantes, au nombre de 23 en $10 \mu$. ")

Il décrit également deux variétés: var. promiscula, linéaire, à extrémités rostrées; puis, var. producta, 
linéaire lancéolée, à extrémités rostrées; longueur de la valve 30 ì $40 \mu$. C'est à cette dernière variété que ressemble le p'us notre Stauroneis antediluciana.

Stauroneis antediluviana no". sp. (Pl. XI, fig. 6). - De forme largement lancéolée, à extrémités prolongées, fines et arrondies; longueur de la ralve 40 à $50 \mu$, largeur 12 à $15 \mu$; lumen (vue de la cloison par la face valvaire) très visible, paraissant séparer les extrémités prolongées de la valve; stauros rectiligne, étroit et très marqué; aire hyaline axiale très étroite, s'élargissant près du stauros; stries très fines, à peine visibles dans le baume.

Differe du Stauroneis (Pleurostauron) parrula Grun. par sa taille plus grande et par sa forme plus largement lancéolée; se distingue du Stauroneis (Pleurostauron) Frauenfeldiana Grun. par sa taille plus petite, sa forme plus trapue et son stauros rectiligne.

Stauroneis acuta W. Sm. (Diat. d'Aur., p. 78).

Stauroneis javanica Grun. var. arvernense nov. (Pl. XI, fig. 8). - Talre largement lancéolée, à extrémités prolongées et arrondies; longueur 120 i $150 \mu$, largeur 25 à $30 \mu$; stries au nombre de 11 en $10 \mu$, formées par des grantiles légìrement allongés, au nombre de 10 en $1.0 \mu$ dans le sens de la longueur de la valre; lumen très visible, se raccordant à la valve à environ 1/3 de l'extrémité au centre. - Diffère du Stauroneis (Pleurostauron) jaranica Grun. (Grun. 1lg. Nor., pl. 1, fig. 14) par son stauros non linéaire, par ses stries 
plus écartées et formées de granules p'us gros. - Le type n'est connu qu’ì Java.

Stauroneis Baileyi Ehrb. (Ehrb. Amer, 1. 143). - Analogue comme forme générale, an Sturroneis Phonicen'eron Ehrb., mais à striation plus large et plus distinctement granulée.

Stauroneis Thenicenteron Ehrb. (Diat. d'Auv.,p. 75). - gallica M.Per.F.Hér. (Diat.d'Aur.,p.77). - anceps Ehrb. (Diat. d'Aur., p. 7i).

Stauroneis anceps Ehrb. var.linearis Grun. - Tout à fait conforme à la figure 8 pl. 4 du Symopsis de Tan-Heurck.

Diploneis elliptica Ktz. (Thiat. d'Aur., p. 10t).

- - var.oblongella Sceg.(D.d'Auc., p. 105).

Amploora affinis Ktz. (Diat. d'Auv., p. 63).

-_ distincta nov. sp. (Pl. IX, fig. 2).

L'aspect d'un Ampliora étant très différent, suivant la position du frustule ou de la valve isolée dans la préparation, et sachant, par expérience, la difficulté que les débutants en diatomologie éprouvent pour acquérir la notion exacte de la structure de cette Diatomée, nous domnons ici une épure, représentant les direrses positions de notre Amphora Borneti.

Soit $O$, le rabattement de la section transrersale de l'Amphora, faite perpendiculaire au grand axe du frustule, et prasssant près du nodule central; $\mathrm{V}$ et $\mathrm{V}^{\prime}$ sont 


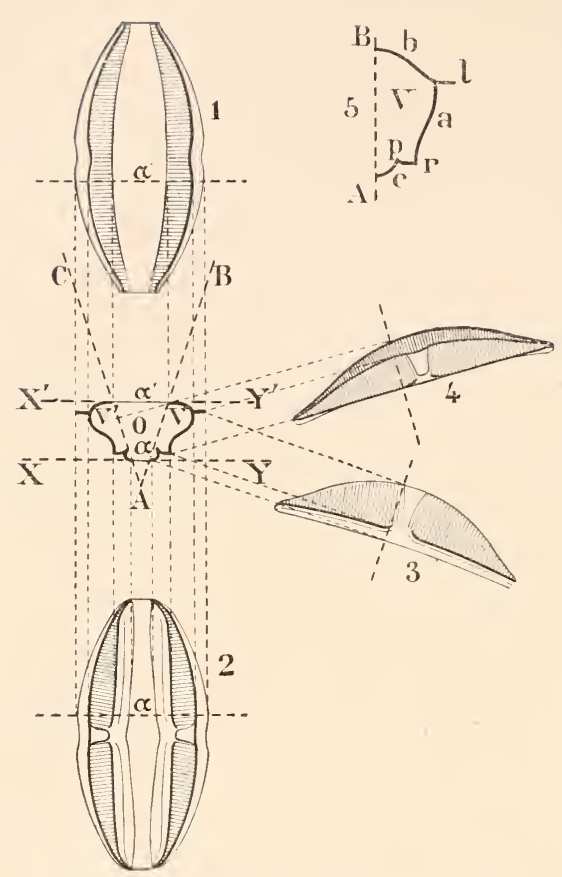

Structure de l'Amphora Borneti F. II rib. (Dessin du Frere Charles) $\frac{600}{1}$

les coripes des deux valves (figurées par un trait fort);

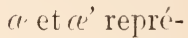
sentent les coupes de l'anneau connectif; fig. 1 , face dorsale; fig. 2, face ventrale du frustule.

D'après cette coupe, on voit qu'en définitive un Ampleoran'est pas constitué a utrement que les auires Dia to mées Raphidées; chaque ralve étant formée par trois surfaces, ainsi que le montre la fig. $\check{\delta}$, représentant la valve $\mathrm{V}$ de la figure $\mathrm{O}$.

Le côté dorsal est indiqué par $a$ et $b$, le côté ventral par $c$ : le rapné est rejeté en $r$, sur l'arête du bord ventral de la valve, l'autre bord étant occupé par une aile l, plus ou moins visil,le, qui peut même manquer. Le frustule a subi une constriction sur un côté, d'où :

$1^{\circ}$ Valve crmbiforme plus ou moins prononcée. 
$2^{\circ}$ Différence de largeur de l'anneau connectif en ce et $a^{\prime}$ (fig. O), le côté ventral $\alpha$ étant beaucoup plus étroit que le côté dorsal ce'; c'est à la même cause que l'on doit rapporter le pli $\mathrm{P}$ (fig. 5) du côté ventral de la valve.

C'est encore ì la déformation de la valve que l'on doit les vues si différentes d'un Amphora, suivant la position qu'il occupe dans la préparation.

Quand le frustule est entier, il se place, soit sur la face rentrale selon XY, soit sur la surface dorsale selon X'Y', d'où les deux vues, fig. 1 face dorsale (Pl. XI, fig. 3), fig. 2 face ventrale (Pl. XI, fig. 1); on observe plus facilement la vue fig. 2 parce que les raphés, ordinairement très risibles, et la partie centrale hyaline, se projettent sur l'anneau connectif large de la face dorsale ; c'est également la rue la plus commune, car elle corres. pond à la position la plus stable du frustule entier.

Si le frustule est désagrégé, il se présente deux cas :

$1^{\circ}$ La valve est complètement isolée, elle repose alors sur ses deux bords ou sur la surface $a$ (fig. 5 ), qui lui est sensiblement parallèle, elle se projette sur le plan AB (fig. 0 et 5), et on apergoit le raphé sur le bord concave, le côté ventral de la valve, ordinairement en raccourci, et qui se place en dehors du raphé, quant au côté dorsal, il est presque toujours invisille; l'aile $l$ (fig. 5) est tellement liyaline qu'elle disparaît dans la striation de la valve; c'est la rue fig. 3 , représentée aussi (Pl. IX, fig. 1); cette position, de la valve isolée, est la 1.hus ordinaire et la plus stable.

$2^{\circ}$ La valve reste adhérente au connectif; dans ce cas elle se projette sur le plan $\Lambda \mathrm{C}$ (fig. $\mathrm{O}$ ); la valve est inclinée et elle se présente par l'angle dorsal; c'est alors la rue fig. 4, représentée aussi (Pl. XI, fig 2); on voit la paroi dorsale de la valre, et le raphé situé tout ì fait sur le bord rentral; la paroi ventrale a disparu, parce 
que, dans cette position, elle peut être perpendiculaire au plan de rision, ou situce sous la valve.

Après la description sommaire de la structure et des vues différentes que présente l'Ampliora Borneti, voici la diagnose de cette espèce.

Amphora Borneti F. Hérib. (PI. IX, fig. 1 et Pl. XI, fig. 1, 2 et 3$)^{(1)}$. - De taille assez petite, lungueur de la valve 50 à $70 \mu$; face valvaire cymbiforme, large de 10 à $15 \mu$; côté dorsal très convexe, et côté ventral légèrement concave; valve à extrémités faiblement prolongées et arrondies; raphé presque droit, et dont les extrémités portent deux petitos ailes en forme de spatule; nodules petits, les centraux légèrement récurvés vers la face dorsale; aire hyaline axiale notahle au milieu de la partie dorsale, diminuant rapidement de largeur vers les extrémités, non dilatée autour du nodule médian ; stauros large, à flancs rectilignes, ou légìrement courbes, s'élargissant du côté dorsal; stries convergentes, légèrement courbes, au nombre de 12 en $10 \mu$, composées de granules allongées, formant des lignes longitudinales sinueuses. Les stries ne sont pas interrompues sur le stauros, mais elles y sont moins marquées, et décroissent d'intensité du bord dorsal vers le nodule médian; bord dorsal ailé; l'aile n'est pas visible sur la vue valvaire, mais on l'apereoit distinctement sur la rue connective; côté rentral présentant un pli interrompu

(1) La difference que lon constate dans les dimensions proportionnelles des dessins relatifs à cette espèce, est due à ce que I-s frustules, entiers ou desagrégés, examinés dans les préparations, n'étaient pas absolument de même granleur; ainsi les deux valres isolées (l'l. IX, tig. 1 et Pl. XI, fig. 2) proviennent de deux frustules de taille differente.

Cette observation sapplique à la plupart des especes reprcisenties par plusieurs dessins. 
devant le nodule central et accompagné de courtes stries; vue connective ovale, ì extrémités rostrées, fortement tronquées, montrant les ailes dorsales et terminales du raphé. Zone simple.

Cette espìce, très remarquable, et assez fréquente dans le dépôt, est dédicie au savant algologue français, II. le $\mathrm{D}^{\mathrm{r}} \mathrm{Ed}$. Bornet, IIembre de l'Institut.

\section{Cymbella cymbiformis Ehrb. (Thut. d'_lur., p. 69). \\ - Tuengarica Pant. (Pant. Lng. II, pl. 1, fig. 14). \\ - Brecieri F. Hérib. Pl. IX, fig. 12).}

Cymbella Pagesi F. Hérib. (Pl. IX, fig. 14) Frustule de grandeur moyenne, longneur $100 \mu$, largeur $19 \mu$; valve à côté dorsal fortement et régulièrement arqué, côté ventral légèrement concave et faiblement gibbeux; extrémités larges et arrondies, un peu récurvées ver's la face dorsale; raphé occupant sensiblement le milieu de la valve, fortement et régulièrement arqué; nodules terminaux en flamme, récurrés vers la face dorsale et $\mathrm{y}$ aboutissant; aire hyaline axiale nulle sur la face dorsale, linéaire et très étroite sur la face ventrale; aire hyaline centrale allongée et trìs faible sur la face dorsale, assez forte sur la face ventrale; stries fortes, très finement divisées en travers, faiblement radiantes jusque près des extrémités, où elles deviennent assez rapidement radiantes et plus serrées; stries dorsales au nombre de 6 en $10 \mu$ au milieu; stries ventrales au nombre de $S$ en $10 \mu$, excepté aux extrémités et au milieu de la valve, où elles ont l'écartement de 11 en $10 \mu$.

Diffère du Cymbella cymbiformis Ehrb. par sa grande taille, par sa plus forte courbure et par ses extrémités plus larges et un peu récurrées. 
Tous dédions ce Cymbella ì MI. Pagìs-Allary; c'est dans l'un de ses envois que nous l'arons découvert.

Cymbella gastroides Kitz. (Diat. d'Aur., p. 68)

- aspera Ehrb. (Diat. d'Aux., p. 69).

- cuspridata Ktz. (Diat. d’.4ur., p. 65).

- Laulıyi F. Hérib. Pl. IX, fig. 4).

- capitata M. I. et F. H. (PI. IX, fig. 6).

Cymbella Hauckii V. II. var. fossilis no: Pl. XI, fig. 4). - Se distingue du Cymbella Hauclii I. I. (Clève, Sym. 1891, pl. 4, fig. 24), par ses extrémités légèrement rostrées, par la forme de son aréa, et par les stries moins serrées, 8 en $10 \mu$ au milieu de la partie dorsale et 10 en $10 \mu$ au milieu de la partie rentrale.

Arons-nous lia Cymbella Lyndsayania Grev.? d'apris Clève (loc. cit.), cette espèce a aussi les stries plus écartées que celles du ('ymbella IIauckii; nous n'avons pu vérifier le fait, n'ayant pu nous procurer la Diato. mée de Greville.

Cymbella leptoceras Ḱtz. forma curta (D. d' Auv, p. 22:0)

Cymbella Rhodesi F. Ilérib. (PI. IX, fig. 1f). -- Grande et belle espèce, à valve de forme lenticulaire, ì extrémités petites, prolongées et arrondics; longneur $120 \mu$, largenr $38 \mu$; raphé fin, formé de deux lignes droites; nodules petits, les terminaux fourchus, les centraux en forme de crochets tournés vers le bord ventral; aire hayline axiale lancéolée, large, arroudie autour du nodule médian, plus fortement du côté ventral que du côté dorsal; stries radiantes, fines mais bien marquées, d'autant plus inclinées et plus serrées qu’elles 
s'éloignent du milieu de la valve, où elles sont au nombre de 6 en $10 \mu$ ì la partie dorsale, et de $7 \frac{1}{2}$ en $10 \mu$ à la partie ventrale; finement mais distinctenent divisces en travers.

Diffère du Cymbella cuspiclatu Ǩtz. par sa taille plus grande, par ses extrémités moins hrusquement diminuées, et par son aire liraline axiale beaucoup plus grande.

Nous dédions cette belle espice à notre trìs distingué compatriote, M. Riliodes, associé de M. Pagès, pour l'exploitation industrielle des riches dépôts à Diatomées du Cantal.

Gomphonema rigidum nov. sp. (Pl. I, fig. 1). - De taille moyeme, longuem $\delta 0 \mu$, largeur $13 \mu$; valve étroitement lancéolée, d̀ extrémités fines, arrondies; aire hyaline axiale lancéolée, tr’’s étroite, légèrement arrondie d'un côté du nodule médian, et de l'autre autour d'un point unilatéral bien visible; stries très légìrement convergentes, non distinctement granulées, au nombre de 11 en $10 \mu$, et non sensiblement plus serrécs aux extrémités qu'aũ milieu de la valve.

Diffère du Gomplionema dichotrmum II. Sm., dont il a la forme générale, en ce que les stries médianes ne sont pas plus écartées, ni beaucoup plus courtes que les leux voisines, et que, par suite, l'aire hyaline centrale n'est pas stauronéiforme.

Gomphonema Vibrio Ehrb. (Diut. d'Aur., p. 5:). affine Ktz. (Diat. d'Aur., p. 60).

Gomphonema clavatum Grun. forma curta I. il. (V. H. Syn., pl. 23, fig. 12). Cette forme n'était encore connue qu'à l'île Cuba.

Gomplionema commutatum Grun. (D. d'Aux., p. 55). 
Gomphonema eriense Grun. (V. II. Synn., pl. 23, fig. 10) ; trouré vivaut dans le lac Erié, Amérique du Nord.

Synedra ('Tna Ehrb. (Diat. d'Aur.,p. 137).

- - var. amphirhynchus Ehr. (D.d'Aur., p.150)

Fragilaria nitida F. Ilérib. et M. Per. var. minor nox. - Semblable au trpe décrit dans le dépôt de Joursac, mais beancoup plus court tout en étant aussi large, longueur 30 à $50 \mu$.

Fragilaria nitida F. H. var. delicatula nov. (PI. II, fig. 29) - brevistriata Grun. (Diat. d'Aux., p. 146).

Fragilaria brevistriata rar. elliptica nov. (Pl. X, fig. 11). - Forme générale du Fragilaria elliptica Schum., mais avec la striation fine et marginale da Fragil. brevistriata Grun.

Fragilaria construens Grun. (Diat. d'Aur., p. 143).

- - var. venter Grun. (Diat.d'Aux., p. 14t).

- elliptica Schum. (Diat. d’Auv., p. 145).

- intermedia Grun. (Diat. d'Auv., p. 146).

Fragil. Lancettula Schum (V.H. Syn.,pl.45,fig.20)

Fragilaria Zeilleri F. Hérib. (Pl. X, fig. 9). - N'est pas tout à fait identique à la forme de Joursac; le milieu de la valve est un peu moins large et les extrémités plus épaisses.

Eunotia parallela Ehrb. (Diat. d'Aur., p. 133).

- impressa Elrb. (Diat. foss. d'Aur., p. 27).

- pecinalis Rab. (Diat. d'Aue., p. 132). 
Epithemia Sorex Ktz. (Diat. d'Aur., p. 126).

- turgida Ǩtz. (Diat. d'Aur., p. 124).

- - var.granulataGr. (Diat.d'Auv.,p.125)

- Zebra Ktz. (Diat. d'Aur., p. 127).

- - var.proboscidea Gr. (Diat.d'Aur.,p.128)

Grunouia Tabellaria Grun. (Diat.d'_tur., p. 166).

Grunowia moissacensis nov. sp. (Pl. X, fig. 25). - Valve en forme de losange allongé, à extrémités légèrement capitées, un peu récurvées du côté opposé à la carène; longueur $40 \mu$, largeur $6 \mu$; côtes allant jusqu'au milieu de la valve, an nombre de $5 \frac{1}{2}$ à 6 en $10 \mu$ au milieu de la valve, plus serrées aux extrémités; stries fines, au nombre de 20 en $10 \mu$.

Diffère du Grunouria simuata Rab. par ses côtes non ondulées, et du Grunouria Tubellaria Rab. par sa tail!e et par sa forme.

Cymatopleura Martyi F. Hérib. (Pl.X, fig. 26). -- Aspect général du C'ymatopleura elliptica IV. Sm. var. sulconstricta Grun. (V. H. Syn., pl. 55, fig. 2); longueur de la valve 110 à $133^{\prime \prime} \mu$, largeur $4^{\prime}$ ) à $50 \mu$; granules au nombre de 3 en $10 \mu$, écartés les uns des autres d'environ leur diamètre; stries très difficilement risibles dans le baume; une forte ondulation, bien marquée, partant du point oì commencent les extrémités, une deuxième plus faible, quelquefois invisible, entre la première et le centre. La convexité de ces ondulations cst tournće vers les sommets de la valve.

Diffère du C'ymatopleura elliptica var. subsconstric'a Grun. par ses extrémités coniques et subrostrées, par la disposition inverse des ondulations, c'est-ì-dire à convexité touruée vers les extrémités, tandis qu'elle est 
tournée vers le centre dans le Cymatopleura elliptica et ses variétés. - Se distingue du Cymatopleura Solea Bréb. par sa torme plus large et moins acumiuée, par ses granules plus espacés et par le petit nombre de ses ondulations.

Nons dédions cette belle Diatomée ì M. Pierre Marty, notre savant collaborateur, pour la publication prochaine de la Flore mincène de Joursac.

Surirella gracilis Grun. (Diat. d'_tux., p. 180).

- splendida Ehrb. (Diat. d'Auv, p. 175).

- tencra Greg. (Diat. d'Aux., p. 180).

Opephora Martyi F. Hérib. (Diat. foss. d'_tux., p. 43) - - var. capituta nov. (I'l. X, fig. 16).

Dans le dépôt de Iloissac la variété est beaucoup plus fréquente que le trpe.

Diatsma pectinale Kitz. (Diat. d'_lux., p. 151).

Diatoma pectinale Ktz. var. capitata nor. Nous dénommons ainsi les formes semblables à celles représentées par Yan-Heurck dans son synopsis, pl. 50, figures 24 et 25 .

Tetracyclus emarginatus WV. Sm. (D. d'Aur., p. 158).

Melosiru undulatu Kitz. (Diut, foss. d'Aur., p. 22).

- - var. producta A. Sch. (Diat. foss. dAur., p. 2.2).

- - var. debilis nov. (Pl. X, fig. 27).

- gramuluta Erlib. (Jiat. d's Lue, p. 186).

Nuns arons ol serré en rue connective et figuré (Pl. X, fig. 28), un frustule formé par la réunion d'une ralve de la rariété debilis et d'une valve de la variété productu; 
fait curieux pour servir à l'étude de la formation des variétés.

Melosira lineolata Grun. var. robusta nor. (Pl. X, fig. 33). - Diamètre de 10 ¿̀ $18 \mu$; face valvaire lisse à l'exception d'une fine crénulation marginale; face connective d'une longueur à peu près constante de $1 \check{u}$, et par conséquent plus longue que le diametre dans les petits exemplaires, et plus courte dans les grands; stries granulces, au nomlire de 12 ì $13 \mathrm{ell}$ 10 i , ordinairement sinueuses, en forme d's allongé, normales au plan des valves et obliques au bord du connectif, formées de grauules séparés, fins et bien visilles ; frustule assez robuste, ¿̀ cloison forte, et dont l'ourerture est assez étroite.

Diffère du Melosira lineolata Grun. (T.II. Synn. Pl.88, fig. 1 et 2), par sa face valvaire lisse, par ses stries plus fortes et moins serrées, et surtout par l'aspet général plus vigoureux. Differe dn Melosina lypata var. lacustris forma temior (V. H. Syn. pl. 87, fig. 3, 4,5) par la forme des parois du frustule qui est la caractéristique de cette dernière espèce.

Melosira Taxis Grun. (Diat. d' Aur., p. 187). Reseana Rab. (Diat. d'Auv., p. 185).

Cyclotella perforata nov. sp. (Pl. X, fig. 35).

- Tiris F. Hérib. (Diut. d'itur., p. 224).

Cyclotella Kutzingiana Chauv. var. gemmatulata nov. (PI. X, fig. 36). - Longueur du diamètre 20 à $30 \mu$; face valvaire striće sur environ la moitié du rayon; stries fortes, à peu près alternativement longues et courtes, ayant à leur base, près du hord de la valve, un renflement allongé formant presque une perle; 10 à 12 stries en $10 \mu$ près du bord de la ralve; centre 
lisse ou très légèrement maculé, présentant quelquefois au centre de la valve un point plus ou moins risible; valve très bombée du bord au centre.

Cette variété pourrait être prise pour une grande forme de notre C'yclotella perfuruta, mais elle s'en distingue facilement par ses stries plus égales, par le relief de la valve, et surtout par l'alsence du trou caractéristique du Cyclotella perforata.

Les espèces caractéristiques de Moissac sont: Navicula ma,or, Epithemia Ilyndmannii, Melosira granulata et canalifera, Cyclotella Iris et perforata.

Ce beau dépôt se place immédiatement après ceux de Joursac et d'Auxillac, pour la richesse de sa florule diatomique; le nombre des espèces et rariétés qu'il nous a livrées dépasse 130, parmi lesquelles près d'une quarantiine sont inédites et bien définies. 


\section{DÉPOT DE NEUSSARGUES}

Les fondations d'une maison récemment élevée sur l'emplacement même du dépôt de Neussargues, ayant mis à jour l'épaisseur totale du dépôt, nous avons profité de cette circonstance pour compléter la liste des Diatomées déjà connues.

Les échantillons étudiés, nous ont livré les espèces et variétés suivantes :

Cocconeis Placentula Ehrb. (Diat. d'Aur., p. 4t).

Saricula acrospharia Bréb. (Diut. d'Aluc., p. 93).

- sculpta Ehrb. (Diat. foss. d'Auc., p. 41).

Navicula peregrina Heib. var. fossilis nov. (Pl. XI, fig. 21). - Valve longuement lancéolée, ¿ extrémités arrondies, longueur $90 \mu$, largeur $18 \mu$; stries très distinctement divisées en travers, radiantes jusque près des extrémités, où elles sont perpendiculaires au raphé, au nombre de 7 en $10 \mu$, les médianes un peu plus écartées et non alternativement longues et courtes, laissant une aire hyaline axiale ćtroite, s'élargissant progressivement depuis les extrémités jusqu'au nodule médian, où elle est en forme de losange; la strie médiane qui aboutit à la pointe de ce losange est plus courte que la moitié de la largeur de la valve.

Differe du type par ses stries plus serrées, et par la forme de son aire liyaline centiale. 
Iraricula meniscutus Sthum. (Diat. d'Aur., p. 98).

- ratiosa Ktz (Diat. d Aur., p. 99).

- rupestris Ktz. (Diat. d'Auv., p. 81).

- major Ktz. (Diat. d'Auv., p. 82).

Jiploneis clliptica Kitz. (Diut. d'Aur., p. 101).

P'eurosigma acuminatum (ir. (Diat. d'Aur., p. 122).

Ampleora oratis Kiz. (Diat. d'Aur., p. 62).

Cymbella aspera Ehrb. (Diat. d'_lux., p. 69).

- Pautill. P'er. F. Hérib. (Tiat. d’Aux., p. ro.)

Encyonema centricosum Kiz. (Diat. d'Auv., p. 73).

Gomphonema brevistriata nor. $s p$. (Pl. XI, fig. 22). - Frustule de taille moyenne; longueur de la valve $73 \mu$, largeur $11 \mu$; de forme biconique, à extrémités arrondies; stigmate unilatéral petit, mais bien visil.le; stries non distinctement granulées, rayonnantes d'autant plus qu'elles s'éloignent du centre, au nombre de 11 en $10 \mu$, excepté pour les trois stries centrales qui sont beaucoup plus écartées; aire hyaline axiale assez large, très légèrement conique, largement arrondie autour du nodule médian, oì les stries centrales sont presque marginales.

Espèce lien caractérisée par son aire lyaline centrale trìs large.

Gomphonema subclaratum Grun. (Diut.d'Aur., p.55)

Synedra Ramesi F. Hérib. (Pl. XI, fig. 28). Diatomće de petite taille; valve légèrement étranglée au milieu, ì extrémités atténućes, rostrées, aiguës, longueur $50 \mu$, largeur $8 \mu$; stries fortes, non distinctement granulées, au nombre de 10) en $10 \mu$, parallèles, excepté aux extrémités où elles sont légèrement courbes et 
rayonnantes, laissant entre elles un pseudo-raphé étroit, mais très visible, et une aire hyaline centrale très grande (longueur 10u), tenant toute la largeur de la valve.

Nous dédions cette Diatomée au très regretté géologue cantalien, J.-B. Rames, en souvenir de l'intérêt affectheux qu'il prenait à nos modestes travaux sur la flore d'Auvergne.

Synedra LTha Ehrb. (Diat. d'Aur., p. 137).

- - var. amphirhynchus. (D. d'Aur., p. 138)

- - var. danica (Diat. d'Aux., p. 138).

Fragilaria elliptica Schum. (Diat. d'Auv., p. 145).

Eunotia polyglynthis Ehrb. (Diat. d'Auv., p. 134).

Epithemia Sorex Ktz. (Diat. d'Auv., p. 126).

- Zelira Ktz. (Diat. d’Aur., p. 127).

- - var. minor nov. (Diat. d'Aur., p. 129).

- - var. proboscidea Gr.(Diat.d'Auv.,p. 128).

- turgida Ktz. (Diat. d'Aur., p. 124).

Rhopalodia gibba E. var. ventricosa Gr. (D.d'A.,p. 126)

Surirella splendida Ehrb. (Diat. d'Auv., p. 175).

Diatoma hyemale Heib. var. acuta nor. (Pl. XI, fig. 7). - Differe du type par sa forme lancéo. lée, ì extrémités aiguës et légèrement subrostrées; stries intercostales assez visibles.

Tetracychus emarginatus W. Sm. (Diat.d'_Auv., p. 158)

Melosira tenuissima Grun. (Diat. d'Auv., p. 188).

Les espèces observées le plus souvent sont: Cocconeis Placertula, Eunotia polyglyphis et Melosira temuissima, très commun dans plusieurs des échantillons étudiés. 


\section{RÉSULTATS ACQUTS}

Les argiles miocènes à Diatomées du Cantal, dont nous venons de terminer l'étude, nous ont livré 1:7 espèces et variétés à ajouter à la flore d'Auvergne, parmi lesquelles 114 sont nouvelles pour la flore générale.

Voici la liste des formes inédites et figurées dans les quatre planches de ce mémoire :

Achnanthes joursacense nor. $s p$. (Joursac).

- Flahaulti nor. sp. (Moissac).

Cocconeis lineata Grun. rar. rotunda noc. (Joursac).

- Bonnieri nov. sp. (IIoissac).

Navicula gibbula Clère (Moissac).

- - var. cantalica nor. (Moissac).

- Pupula Kitz. var. minor nor. (Andelat).

- pseudo-bacillum Grun. var. elapsa nov. (Joursac).

- ventricosa Donk. var. decrescens nor. (Joursac).

- - var. cuneata nou. (Moissac).

- Iridis Eurb. rar. subproducta nor'. (Moissac).

- dubitata nov. sp. (Joursac).

- Gendrei nov. sp. (Joursac).

- Chaberti nor. sp. (Moissac).

- Placentula Ehrb. var. major nov. (Andelat).

- acephala nov. sp. (Moissac).

- joursacensis nov. sp. (Joursac).

-- Reinhardtii Grun. var. elliptica nor. (Joursac).

- triangulifera nor.sp. (Joursac).

- digito-radiata Greg. rar. obesa nov. (Joursac).

- Costei nor.sp. (Jonrsac).

- - Far, bacillaris nov. (Joursac). 
Naricula peregrina Heib. var. fossilis nov. (Nenssargues).

- major Ǩtz. rar. convergentissima nov. (Joursac).

- Esox Ehrb. var. recta nor. (Joursac).

- Olivieri nor. sp. (Joursac).

- decurrens Ehrb. var. curtecostata nov. (Joursac).

- Leveillei nov. sp. (Joursac).

- Huei nor. sp. (Moissac).

- microstauron (Ehrb.) (Moissac).

- divergens W.Sm. var. undulato-cuneata nor. (Moissac)

- decrescens nor.sp. (Moissac).

- gracillima Pritch. var. lucida nor. (Joursac).

- bicapitata Lag. var. sulcata nov. (Moissac).

- Bramii Grun. var. moissacensis no". (Moissac).

- acrospheria Bréb. var. bacillaris nov. (Moissac).

- - var. elongata nov. (Moissac).

- hemiptera Kítz. rar. angusta nov. (Moissac).

- Recauldi F. Hérib. var. major nor. (Joursac).

- Berriati nor.sp. (Joursac).

- - var. minor noz. (Joursac).

Stauronc is quadrata nor. sp). (Joursac).

- parrula Grun. (Moissac).

- jaranica Grun. var. arrernense nov. (Moissac).

Amphora affinis Ktz. (Joursac).

- Bornctinor. sp. (Moissac).

- distincta nor. sp. (Joursac).

Crmbella Harioti nor. sp. (Joursac).

- radiosa nor. sp. (Joursac).

- hungarica Grun. (Joursac).

- Brevieri nor.sp. (Joursac).

- Foncaudi nov. sp. (Joursac).

- Pagesi nor. sp. (Moissac).

- Creguti nor. sp. (Joursac).

- Hauckii V. H. rar. fossilis nov. (Moissac).

- meniscus nor. sp. (Joursac).

- Laubri nor. sp. (Joursac).

-. - rar. lanceolata nor. (Moissac).

- capitata nov'sp. (Faufouilhonx).

- Rhodesi nov. sp. (Moissac). 
Encyonema cespitosum Ḱtz. var. Anerswaldii V. H. (Joursac).

- intermedium nov. s, (Joursac).

- Grandi nov. sp. (Joursac).

Gomphonema rigidum nov: sp. (Moissac).

- latestriata nor. sp. (Joursac).

- cliratum Ehrb. var. curta nov. (Moissac).

- micropis Ktz. rar. major nor. Joursac).

- eriense Grun. (Joursae).

- - var. aciminata nor. (Joursac).

- insigne Greg. var. minor Grun. (Joursac).

- semiapertum Grun. (Joursac).

- breristriata nov. $s p$. (Neussurgnes).

- $\quad$ exscissum nor: $s p$. (Jour:ac).

- parrum nor. sp. (Joursac).

- accessum nor. sp. (Joursac).

- Gilloti nov. sp). (Joursac).

- $\quad$ arcticum Grun. (Joursac).

- Licmophoraoides nol. $s \%$. (Joursac).

Synedra jour'sacensis nor'. sp. (Joursac).

- Ramesi nov. sp. (Neussargues).

Fragilaria brevistriata Grun. rar. elliptica nor. (Moissac).

- Harrisonii Grun. rar. major nor. (Joursac).

- Gustarei nov.sp. (Joursac).

- construens Grun. var. circulare nov. (Joursac).

- minutissima Grun. (Joursac).

- Lancettula Schum. (IIoissac).

- Zeilieri nov.sp. (Joursac).

-_ - - var. nitzschioides nov. (Jour'sac).

-- - forma anomala nor. (Joursac).

- bidens Heib. (Joursac).

- nitida nor'.sp. (Joursac).

- - var. delicatula nov. (Joursac).

- - rar. minor nov. (ILoissac).

Actinella pliocenica F. Hérib. var. tenuistriata nox'. (Chambeuil).

- - rar. serpentina nor. (Fraisse-Bas).

Eunotia polydentula Ehrb. var, fossilis nov. (Joursac). 
Epithemia Hyndmannii W. Sm. rar. perlonga not. (Joursac). turgida Kitz. var. porcellus nor. (Faufouilhoux).

Grunowia moissacensis nov $s p$. (Moissac).

Cymatop'eura Martyi nov. sp. (Moissac).

Surirella gracilis Grun. var. constricta nor. (Joursac).

- - var. eximia nov. (Sainte-Anastasie).

- Pagesi nor. sp. (Joursac).

Opephora Martyi F. Hérib, var. capitata nov. (Joursac).

- - var. robusta nov. (Andelat).

- cantalense nor. sp). (Joursac).

- - var. capitata nov. (Joursac).

Diatoma pectinale Ǩtz. var. capitata nov. (Moissac).

Tetracyclus stellare nor. sp. (Joursac).

-. Boryanus (Pant.) rar. minor nov. (Faufouilhous).

- Peragalli nov. sp. (Joursac).

- $\quad$ - var. eximia nov. (Joursac).

- _ - var. major nov. (Joursac).

Melosira undulata Kitz. var. hungarica A. Sch. (Joursac).

- - var. debilis nov. (Joursac).

- lineolata Grun. var. robusta nov. (Moissac).

- imperfecta nor. sp. (Joursac).

- minuta nor: sp. (.Joursac).

- Camusi nol. sp. (Joursac).

- - rar. conica nor. (Joursac).

- canalifera F. H. var. andastomosans nor. (Faufouilhoux).

- granulata Ehrb. var. australiensis V. H. (Joursac).

Cyclotella perforata nou. sp. (Joursac).

- Kutzingiana Cl. var. gemmatulata nov. (Moissac).

Coscinodiscus promens F. II. var. miuutissima nov. (Joursac).

- dispar. F. H. var. inermis nov. (Faufouihous).

En 1883, date de nos premières recherches sur les Diatomées yivantes et fossiles de notre province, les diatomistes attribuaient 122 espèces et variétés à l'Auvergue. 
En totalisant aujourd'hui les Diatomées mentionnées dans nos trois mémoires, nous trouvons qu'elles sont au nombre de 908 , comprenant 564 espèces de premier ordre et 344 variétés bien définies, dont 125 environ sont élevées au rang d'espèces de second ordre par la majo. rité dẹs diatomistes.

Le nombre des formes inédites est de 281, décrites avec soin et figurées dans douze bonnes planches.

Tel est l'état actuel de la Flore diutomiqued' Aucergne, et, en même temps, le résultat de nos labeurs.

Dans les pages suivantes, nous allons donner, it titre de documents pour la flore diatomique du Platean Central, le résultat de la revision des dépôts miocìnes de la Haute-Loire et de l'Ardèche. 


\section{DÉPOTS DE LA HAUTE-LOIRE}

Les dépôts de la Haute-Loire, dont nous avons cherché à compléter la florule diatomique, pour la publication de ce mémoire, contiennent tous des empreintes de feuilles d'arbres; de plus, ils se rattachent l'un à l'autre par l'ensemble de leurs Diatomées caractéristiques; d'où il résulte pour nous que les dépôts de Cerssac, de Vals, du Monastier et de la lioche-Lambert, ne sont que des lambeaux restratifiés par les eaux, et provenant d'un dépôt initial, dont la formation n'a pu s'effectuer que dans un cratère-lac miocèue.

Ces argiles à Diatomées et à plantes fossiles, contiennent, comme celles d'Aurergne, un mélange d'espèces d'eau douce, marines et saumâtres, il faut donc qu'elles se soient formées daus des eaux plus ou moins fortement minéralisées; or, nous verrons plus loin qu’il devait en être ainsi dans les cratères-lacs trachytiques du Plateau Central.

Les florules diatomique et phanérogamique d'un dépôt remanié par les eaux, étant absolument indépendantes l'une de l'autre, quant à leur âge géologique, la seconde ćtant toujours postérieure à la première, il peut se faire, et c'est même probable, du moins pour la Iraute-Loire et l'Ardèche, que la restratification des lambeaux diato- 
mifères de ces régions du Plateau Central se soit opérée à des époques différentes; sur ce point, nous arceptons volontiers la manière de voir des paléontologistes.

\section{DÉPOT DE CEYSSAC}

Le dépôt de Ceyssac, près le Puy, est bien connu des géologues et des paléobotanistes; la florule phanérogamique a été étudiée et publiée par de Saporta, et la florule diatomique, la seule qui nous intéresse, est comprise dans les séries des préparations Tempère.

La liste que nous avons donnée dans les Diatom'es d'Aurergne, page 233, a été établie par M. 'T'empère, et elle nous fut communiquée par notre savant compatriote, II. IIarcellin Boule.

Une autre liste, dressée par notre ami, II. le Commandant DIanrice Peragallo, d'après deux préparations, comprend une trentaine d'espèces. En totalisant les deux résultats, on trouve une quarantaine de Diatomées, attribuées au dépôt de Ceyssac, antérieurement ì notre étude.

La revision de ce beau dépôt a été faite d'après des échantillons nombreux et variés, reçus du F rère Nicolas Albert, professeur au Pensionnat du Puy. Pour éviter les mélanges nous arons eu soin de n'employer que des verres neufs pour nos préparations, au nombre d'une cinquantaine, par conséquent la liste suivante est d'une authenticité absolue :

Achnanthes lancsolata Grun. var. elliptica Clève (Diat. d'Aw., p. 219). 
Achnanthes ligeriana nov.sp. (Pl. XII, fig. פ0). - Diatomée de petite taille; longueur de la valve $15 \mu$, largeur $10 \mu$, largement rhombique arrondie; valve supérieure à aire hyaline axialeétroite, lancéolée; aire centrale unilatéralement développée et érasée, contenant une cloison semi-circulaire, n'ayant comme hauteur que le quart de la largeur de la valve; stries non distinctement granulées, au nombre de 13 en $10 \mu$, courbes, et la courbure tournée rers le centre, celui-ci un peu en dehors des extrémités de la valve; valve inférieure à aire hyaline axiale nulle.

Diffère de notre Achnanthes joursacense (Pl. XI, fig. 26 et 27 ) par sa forme plus large et plus rhombique, par le nombre de ses stries, ainsi que par la cloison latérale qui est beaucosip plus petite.

Coccone is lineata Gr. var.euglypta (Diat. d' A we, p. 46) Sacicula Bacillum Ehrb. (Diat. d'Aue., p. 117).

Navicula Bacillum var. major nox. - Longueur $80 \mu$, largeur $16 \mu$. Clève donne comme longueur de la forme trpe 35 à $55 \mu$, et Tan-Heurck $55 \mu$.

Saricula rentricosa Donk. (Diat. d'Aur., p. 113),

- limosa Kotz.var.gibberula Gr. D.d'A.,p. III).

- Tridis Ehrb. (Diat. d'.luc., p 113).

- cuspidata Kitz. (Diat. d'Aur., p. 107).

Navicula halophila Grun. var. major not. (Pl. XII, fig. 2). - Longueur de la valve $120 \mu$; stries au nombre de 15 en $10 \mu$.

Clève (Synopsis 1894, p. 109) donne pour le type $50 \mu$ de longueur et $19 \AA 20$ stries en $10 \mu$; nous avons donc là une variété bien caractérisée. Le Navicula lialophila Grun. peut être considéré comme une variété 
très étroite, à extrémités non capitées, du Naricula cuspidata Ktz.

Naricula spleceroptiora Ktz. (Diat. d'Aur., p. 109).

- Gendrei F. Hérib. (Pl. IX, fig. 31).

- Placentula Ehrb. (Diat. d'Aur., p. 102).

- dicephala W. Sm. (Diat. d'Auv., p. 103).

- Ticinharedtii Grun. (Diat. d'Aer., p. 102).

- gracilis E. var.neglecta Gr. (1.II.,Syn.,pl.7,fig. 19).

- menisculus A. Sih. (Diat. d'Aux., p. 98).

- radiosa Kitz. (Diat. d'Aux., p. 99).

- major Ktz. (Diat. d’Aur., p. 82).

- mesolepta Ehrb. (Diat. d’Auv., p. 96).

Navicula mesolepta Ehrb, var, Alberti nor. (Pl. XII, fig. 3). - Longueur 80 à $90 \mu$, largeur 12 à $15 \mu$, analogue, comme forme, au Navicula decrescens du dépôt de Moissac (Pl. XI, fig. 19), mais de taille leaucoup plus grande, à extrémités plus capitées, ì côtes plus robustes et plus longues, 7 à 8 en $10 \mu$, interrompues brusquement au milieu de la valve sans qu'elles diminuent progressivement de longueur, laissant autour du raphé une large aréa axiale de forme lancéolée.

Comme aspect extérieur cette Diatomée est intermédiaire entre le Nacicula polyonca Bréb. et notre Naricula meso'epta var. stanroneiformis nor.; le nombre de ses côtes, leur disprosition à la partie centrale, et la forme de l'aréa la distinguent nettement des deux espèces roisines.

Nous dédions ce Naricula au Frère Nicolas Albert, en souvenir des matériaux très importants qu'il a eu l'amabilité de nous procurer, pour la revision du beau dépôt de Ceyssac.

Navicula radians nor. sp. (Pl. XII, fig. 1). - 
Valve étroitement lancéolée, à extrémités arrondies; longueur 120 à $150 \mu$, largeur $20 \mu$; aire axiale étroite, inégalement déreloppés des deux côtés du raphé; aire centrale arrondie; stries centrales mêlées de stries plus courtes, au nombre de 7 en $10 \mu$, radiantes de plus en plus jusqu'aux extrémités de la valve, où elles sont plus serrées qu'au milieu, 9 en $10 \mu$.

Diffëre du Naricula vulpina Ktz. par sa taille plus grande, par ses stries plus écartées, et surtout en ce qu'elles sont radiautes jusqu'aux extrémités de la valve, au lieu d'y devenir convergentes.

Stauroneis acuta Wr. Sm. (Diat.d'Aur.,p. 78).

Diploneis elliptica Ktz. (Diat. d'Auv., p. 104).

- Smithï Bréb. (Diat. d’Auv., p. 104).

Amphora Pediculus Ktz. (Diat. d'Auv., p. 63). - libyca Ehrb. (Diat. d'Alux., p. 63).

Cymbella cymbiformis Ehrb. (Diat. d'Aur., p. 69).

- maculata Ktz. forma curta Gr. (D.d'A.,p.71

- lanceolata Ktz. (Diat. d'Aur., p. 68).

- gastroides KEtz. (Diat. d’Auv., p. 68).

- - var. minor V.-H. (Diat. d'Aur., p. 68).

- cuspidata Ktz. Diat. d'Aur., p. 65).

Cymbella cuspidata Kitz. var. minor nov.Forme trapue, à extrémités très petites, rostrées.

Cymbella leptoceras Ktz. (Diat. d'Aur., p. 66).

Encyonema prostratum Ralfs. (Diat. d'Auv., p. 72).

-. turgidum Grun. (Diat. d'Auv., p. 73).

- caspitosum Ktz. (Diat.d’Auv., p. 73).

- ventricosum Ktz. (Diat. d'Aux., p. 73). 
Gomphonema constrictum Ehrb. (Diat. d'Aur., p. 52).

- acuminatum Ehrb. (Diat. d'Anv., p. 53)

- - var. pusilla Gr. (Diat. d'Auv., p. 54).

- olicaceum Ehrb. (Diat. d'Auv., p. 61).

Synedra delicatissima W. Sm. var. angustissima (V.H. Syn., pl. 39, fig. 10).

- rumpens Grun. var. fiagilarioides (V. H. Syn., pl. 40, fig. 12).

- - var. Meneghiniana (V.H.S'yn.,pl.40, f.13)

-- - var. scotica (T. H. Syn., pl. 40, fig. 11).

- Clna Ehrb. var. vitrea (Diat. d'Auv., p. 137)

Fragilaria Zeilleri F. Hérib. var. densestriata nov. (Pl. XII, fig. 19). - Diffère du type par ses extrémités plus coniques, arrondies, et par ses stries notaklement plus serrées, 15 en $10 \mu$.

Fragilaria brevistriata Grun. var. capitata nov. (Pl. XII, fig. 18). - Semblable à la variété subcapitata Grun. (V. H. S'yn., pl. 45, fig. 33), mais à extrémités nettement capitées.

Fragilaria construens Grun. var. binotis Grun. (T. H. Syn., pl. 45, fig. 24-25).

- - var. venter Grun. (Diat. d'Aux., p. 144).

Fragilaria bigibba nov. sp. (Pl. XII, fig. 16). - Frustule petit; longueur 15 à $25 \mu$; étroit, 4 à $5 \mu$ à la partie la plus large; valve présentant un rétrécissement au milieu et un autre près des extrémités, qui sont, par suite, nettement capitées; stries presque marginales, mais cependant un peu plus longues que dans le Fragilaria brevistriata Grun., au nombre de 12 à 13 en $10 \mu$, lilissant au centre de la valve une large aréa lancéolée. 
Eunotia polyglyphis Ehrb. (Diat. d'Aur., p. 134).

Epithemia Myndmannii Tr. Sm. (Diat. d' Aur.,p.125).

- - var. curta F. Hérib. (Diat. l'Aur.,p. 125)

- Sorex Kitz. (Diat. d'Aur., p. 126).

- turgida Kitz. (Diat. d'Aur., p. 124).

- - var.granulata Gr. (Diat.d'. Aur., p.125

- Zebra Ktz. (Diat.d'Auv., p. 127).

- _ var.minorF. Hérib. (Diut.d'Auv.p.129

- - var. proboscidea Gr. (D.d'Aur.,p.128)

Rhopalodia gibua Ehrb. (Diat. d'Aur., p. 126).

- - var. rentricosa Gr. (D. d'Aur., p. 126).

IIantzschia amphioxys Grun. (Diat. d'Aur., p. 162).

Cymatopleura elliptica II. Sm. var. rhomboiles Gr. - Nous assimilons à cette rariété de Grunow, qui n'a pas été représentée, une forme lancéolée, à extrémités coniques, arrondies, mais non prolongées, par conséquent de forme rhombique, intermédiaire entre la figure de IV. Smith (S. B. D., pl. 10, fig. $80 a^{\prime}$ ) et le Cymatopleura hibernica du même auteur.

Surirella gracilis Grun. (Diat. d'Aur., p. 180).

- orata Ktz. (Diat. d'.1ur., p. 175).

Opephora Martyi F. Hérib. (Diut. foss. d'Auv., p. 43).

Tetracyclus emarginatus W. Sm. (Diat.d'Aur.,p.15\$). - stella F.H. II. P. (Diat.foss.d'Luv., p. 17)

Melosira bellicosa nov. sp. (Pl. XII, fig. 23). - Diamètre très variable, 5 à $18 \mu$, maís de longueur presque constante, 12 à $18 \mu$; cette Diatomée ne peut être mieux comparée qu'à la figure d'une petite balle cylindro-sphérique à culot expansif; la surface de la face connective, depuis le diaphragme jusqu'à l'extré- 
mité, y compris la surface sphérique qui la termine, est converte de stries longitudinales très visibles, au nombre de 10 ¿̀ 11 en $10 \mu$, formées de granules allongés dans le sens de la strie, au nomhre de 7 ì 8 en $10 \mu$; sur la partie sphérique les stries se resserrent et s'atténuent, se pliant à la surface. - Espèce très distincte et très caractéristique, rarement observée en filaments, mais simplement par frustules isolés ou plus souvent par ralves séparées.

\section{Melosira arenaria Moore (Thiat. d'Aur., p. 186). \\ - distans Ehrb. (Diat. d'Aur., p. 185). \\ - - var. scalaris Grun. - Bien conforme à la fig. 30, p. 86 du šym. de Tan-Heurck. \\ - lineolata Grun. (Iliat. d'Aur., p. 188).}

Melosira sphærica nor. $s p$. (Pl. XII, fig. 24).Frustule presque absolument sphérique, d'un diamètre peu variable, 20 ì $25 \mu$; face valvaire présentant un bord finement divisé par 12 à 13 stries punctiformes en $10 \mu$, et une surface couverte de granules assez gros, égaux et épars, au nombre d'environ 7 ì 8 en $10 \mu$; face connective à valves hémisphériques, réunies par un anneau étroit, présentant contre le connectif une bande étroite, composće de petites stries longitudinales distinctement granulées, au nombre de 12 ̀̀ 13 en $10 \mu$; le reste de la surface sphérique est couverte de gros granules épars, au nombre de 7 à 8 en $10 \mu$.

O's. - Cette espèce, quoique sphériqne, appartient sûrement au genre Melosira Ag.; elle établit le passage de ce genre au genre Podosira Ehrb. Comme forme elle ressemble beancoup a notre Ilelosira spiralis var. sphlecrica du dépôt de Celles, qui cst éridemment la forme régénératrice de l'espice, mais ici, nons ne pourons admettre cette hypothèse, car le Melosira splucrica se troure dans les préparations avec denx espèces de Melosira sen'ement: le Mélosira belli- 
c.sa et le Melosira distans rar. scalaris Grun.; or, le Melosira bellicosa a un facies tout différent: les stries sont plus robustes sur la purtie cylindrique, et les granules plus fins an contraire sur la partic sphérique, ce qui est l'opposé chez le Melosira spherica, on ne peut done pas prendre le Melosira spharica comme la forme régénératrice du Melnsira bellicosa. Le Jutosira distans Ehrb, et sa variété sca. laris Grun.. ont plus d'analogie avec le Melosira spherica, mais nous avons pu facilement observer des filaments de ces espices termines par des ralves hémisphériqnes, et elles sont nettement différentes de celles du Melosira spherica, prísentant une structure beaucoup plus fine, et un diamètre toujours sensiblement plus petit; en outre, cette espèce se troure dans les échantillons et les préparations où le Melosira distans on sa variété scalaris sont le moins abondants, ce qui est certainement une contre indication à le désigner pour une valve régénératrice.

Melosira temuissima Grun. (Diat. d'Aur., p.188).

-. varennarum II.P.F.Hérib. (D.d'A.,p. 189).

- varians Ag. (Diat. d'Aur., p. 18t).

Cyclotella comta Ktz. var. radiosa Grun. - Conforme aux figures 1-9 de la pl. 93 du Syn. de Van-Heurck.

Cyclotella comta Ktz. var. decrescens nor. (Pl. XII, fig. 31). - Se ditingue de la variété radiosa Grun. par la forme des granules de ia partie centrale de la ralve qui, au lieu d'être de grosseur ì peu près uniforme et fins, sont, sur un même rayon, de grosseur croissante à partir du centre, et le plus souvent jointifs, formant ainsi de petites côtes granulées cunćiformes.

Cyclotella comta Ktz. var. trinotata nov. (PI. XII, fig. 26.) - Cette variété, de taille en général moins variable et plus petite (10 i $15 \mu$ de diamètre) que les deux variétés précédentes, est caractérisée par la présence, sur la partie centrale de la valve, de trois gros granules (ou excavations) placés contre la partie striée et au sommet d'un triangle équilatéral. Les granules 
des deux ralres ne sont pas opposés entre eux, mais aux intervalles des valves opposées. Ces trois granules sont accompagnés de points beaucoup plus petits qui présentent des positions variables selon les valves; la plus fréquente de ces dispositions consiste en trois groupes de devix points rapprochés, placés perpendiculairement au rayon; dans les petits exemplaires, entre les granules et le centre de la valve et sur les mêmes rayons, ces deux points sont le plus souvent jointifs, et ils forment, entre les granules et le centre, de petites stries très courte's perpendiculaires au rayon et touchent presque les granules; trois points penvent tetre intercalés entre les trois granules, et sur la même circonférence; enfin, les deux syistèmes peurent se trourer réunis sur le même exemplaire. Variété bien caractérisée et constante.

Stephanodiscus Astreca Ktz. (That. d'Aur., p. 193.) - var. minutula Gr. (D.d'A., p. 193).

Les Diatomées que nous avons observées le plus souvent dans les échantillons étudiés sont: Fragilaria construens var. renter, Melosira arenaria et bellicosa, Cyclotella comta var. ractiosa et var. trinotata, Steplianodiscus Astrace, très commun.

Le nombre des espèces et rariétés de la liste précédente est de 85 , parmi lesquelles 13 sont inédites et trìs distinctes. 


\section{DEPOT DE VAIS}

Ce dépôt, encore inédit, est situé près Le Puy, et à proximité du village de Vals. L'échantillon étudié est d'un gris foncé et présente un aspect terreux; les Melosira arenaria et bellicosa, et l'Epithemia Myndmannii s'y trouvent en très grande abondance; les autres espèces sont peu communes on même rares, du moins dans les préparations examinćes.

Toici les espèces observées :

Cocconeis lineata Grun. (Diat. d'Auv., p. 4t).

- - var. eugl!jpta Grun. (D.d’Auv., p. 46).

Naricula major Ktz. (Diat. d’Auv., p. 82).

- amplizola Cl. (Clève, śyn. 1895, p. 45).

- - var.sturoneiformis (D.foss.d'Auv., p. ji)

Diploneis elliptica Ktz. (Diat. d Auv., p. 104).

-- - var. oblongella Nirg. D.d'Aur., p. 104)

Cymbella lanceoluta Ehrb. (Diat. d'Aue., p. 68).

Gomphonema claratum Ehrb. Diat. d'Aur., p. 56).

- subclaratum Grun. (D. d'Aui, p. 55).

Epithemia Hyudmannü W. Sm. (Diat.d'Aur.,p.125)

- turgida Ktz. (Diat. d’Aux., p. 124).

- Westermamï Ktz. (Diat. dAuv., p. 125).

Tetracyclus emarinatus W. Sm. (D.d'Aue., p. 158).

- stella Ehrb. (Diat. fiss.d'Auc., p. 17). 
Melosira arenaria Moor. (Dict. d'Auv., p. 186), - bellicosa nov. sp. (Pl. XII, fig. 23).

Cyclotella comta Ktz. (Diat. d'Aur., p. 191). - - var. recrescens nov. (PI. XII, fig. 31).

Cette liste, établie d'après l'examen d'un seul échantillon, ne nous donne évidemment qu'une partie de la florule diatomique du dépôt; néannoins elle nous permet de constater que Vals se rattache à Ceysac. Il serait important de rechercher les plantes fossiles de ces argiles à Diatomées, et de comparer leur florule phanérogamique à celle de Ceyssac. 


\section{DÉPOT DU MONASTIER}

Le dépôt du Monatier, comme celui de Ceyssac, contient des empreintes de feuilles d'arbres; c'est donc un lambeau du dépôt initial restratifié par les eaux.

La liste suivante a été étahlie d'après une douzaine de préparations, provenant de trois échantillons reçus de M. l'abbé Boulay :

Achnanthes jouracense F. H. et MI. P. (PI. NI, fif. 26 et 27 ) Naricula Chaberti F. Hérib. (Pl. IX, fig. 18).

- Placentula Ehrb. (Diat. d'Auv., p. 102).

- major Ktz. (Diat. d'Au:, p. 82).

-- amplibolu Cl. var. perrieri F. Il. et II.P. (Diat. foss. d'Aue, Pl. VII, fig. 11).

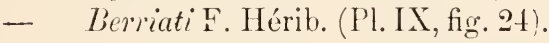

- - var. minor nov. (Pl. IX, fig. 25).

Stuuroneis Phwenicenteron Ehrb. (Diat. d'Auc., p. 75). C'ymbella lanceolata Ehrb. (Diat. d'Auv., p. 68).

- affinis Ktz. (Diat. d'Aur., p. 66).

- Elerenbergï Greg. (Diat. d'Auv., p. 64).

- - var. minor V. H. (Diat. l'Aue., p. 65).

Cymbella Ehrenbergii Greg. var. conica nor. - Nous désignons ainsi une variation qui diffère sensiblement des figures données du Cymbella Ehrenbergï; c'est une forme voisine de celle qui est représentée 
par A. Sch. Atl. pl. 9, fig. 9; mais, dans la Diatomée du Monastier, les extrémités sont encore plus coniques et plus pointues.

Gymbella meniscus F. Hérib. et M. Per. var. major $n \varkappa$. - Se distingue du type (Pl. IX, fig. 5, par sa taille plus grande, et plus ćlancée; par ses stries plus écartées; diffère de notre Cymbella Ellvenbergii var. conica par ses extrémités plus pointues et par l'absence d'aire liyaline axiale.

Cymbella tumidula Grun. (A. Sch., Atl. pl. 9, fig. 3?) Encyonema ventricesum Ktz. (Diat. d'Aur., p. 73).

Fragilaria æqualis Lag. var. capitata nor: - Longueur $24 \mu$; stries an nombre de 15 en $10 \mu$, ne laissant au centre de la valve qu'un psendo-stauros peu visible; diffère du tỵpe surtout par ses extrémités rétrécies et nettement capitées.

Fragilaria Zeilleri F. Hérib. Pl. X, fig. 9). breristriata Grun. (Diat. d'Aue., p. 146).

- _ var. pusilla Grun. (D.d'Aue., p. 146)

- - var. Mormorum Grun. (Diat. d'Aur., p. 14i - construens Grun. (Diat. d'Auv., p. 143). -_ - var. binodis Grun. (V. II. Syll.,pl. 4ü, fig. 24-20) - - var. venter. Grun. (D. d'Auv., p. 144).

Eunotia polyglyplic Grun. (Diat. d'Aux., p. 134).

Epithemia cistula Ralfs. (Pritch, p. 762).

- Hyndmannii W. Sm. (D. d'Auv, p. 125).

- turgida Ktz. (Diat. d'Aux., p. 124).

- - var. granulata Grun. (D. d Aur., p. 12:3)

Rhopalotia gibla Ehrb. (Diut. d'Aux., p. 126). 
Opephora Martyi F. Hérib. (Diat.foss. d'Auv.,p.43). Melosira lineolata Grun. (Diat. d'Auv., p. 188).

- tennis Grun. (Diat. d'Auv., p. 188).

- undulata Ktz. var. producta A. Sch. (Diat. foss. d'Auv', p. 22).

Coscinodiscus pygmocus IL. Per. et F. H. (D. d'ur..p. 194)

Coscinodiscus Boulei I. Per. (Pl. XII, fig. 32). - De grandeur très variable; diamètre de 30 à $100 \mu$; surface légèrement convexe au milieu, sur la moitié environ du rayon de la valve, puis s'abaissant assez rapidement et devenant concave, pour se relever très brusquement tout à fait contre le bord de la valve, présentant ainsi une ondulation concentrique qui le distingue facilement du Coscinodiscus lacustris Grun.; celui-ci présente une ondulation transversale comme la plupart des Cyclotella. Centre très petit, formé de quelques granules épars un peu plus réfringents, quoique sensiblement de même grosseur que ceux qui couvrent le reste de la surface de la valve; ceux-ci sont des points disposés en lignes rayonnantes, formant des faisceaux nombreux dont on n'apergoit les limites qu'à un grossissement assez fort, car ils ne sont pas marqués par des espaces subulés comme dans les Actinocyclus; quelquefois cependant les granules placés à la jonction de deux lignes rayonnautes sont plus gros et plus réfringents, comme dans certains Ilyaludiscus, et la valve présente à un faible grossissement un aspect rugueux ou épineux; les lignes rayonnantes sont au nombre de 10 à 11 en $10 \mu$, et sur ces lignes, on compte 9 à 10 granules en $10 \mu$. Comme dans les Actinocyclus la structure granulaire fasciculée s'arrête arant d'atteindre le bord de la ralye, et en est séparée par une bande étroite 
couverte de points beaucoup plus fins, disposés en lignes décussées; cette bande est peu visible sur les exemplaires entiers, parce qu'elle se trouve sur la partie fortement inclinée qui touche au bord de la valve, mais on la voit bien sur les fragments qui se présentent un peu obliquement. Il existe également un petit nombre d'épines ( 4 à 8 ) intra marginales assez fortes, entre lesquelles on en observe d'autres plus petites et plus nombreuses.

Cette belle espèce est dédiée ì M. Marcellin Boule, professeur de Paléontologie au Muséum.

Les espèces caractéristiques sont : Fragilaria Zeilleri et construens var. venter, Opephora Martyi, Me'csira lineolata et tenuis, Coscinodiscus Mygmaus et Boulei.

La florule diatomique du Monastier, à l'exception du Coscinodiscus Bumlei, est tout à fait analogue ì celle de certains échantillons des dépôts miocènes du Cantal.

D'après les préparations des séries 'T'empère et II. Pe. ragallo, 11 Diatomées étaient attribuées ì ce dépôt; notre liste en mentionne 36 , dont 4 sont nouvelles pour la flore générale. 


\section{DEPÖT DE LA ROCHE-LAMBERT}

Le dépôt de la Roche-Lambert est situé à une dizaine de kilomètres du Puy, sur la ligne d'Arvant; le gisement de ces argiles à Diatomées se troure dans un ravin très escarpé, de difficile accès, sur le flanc droit de la rallée de la Borne, entre la rivière et le chemin qui conduit au château de la Roche-Lambert.

Comme Ceyssac, la Roche-Lambert contient des empreintes de feuilles d'arbres; de plus, les florules diatomiques sont tout à fait analogues, et prouvent que les deux formations diatomifères ne sont autre chose que des lambeaux remaniés par les eaux d'un même dépôt initial.

Les échantillons étudiés, an nombre de quatre, nous ont été communiqués par M. l'abbé Boulay; nous n'avons pas constaté de différence notable de l'un à l'autre; ils contiennent à peu près les mêmes espèces.

L'examen d'une quinzaine de préparations nous a donné la liste suivante, comprenant une soixantaine d'espèces et variétés fort intéressantes, et dont plusieurs sont inédites :

Cocconeis lineata Grun. (Diat. d'Auv., p. 44).

Naricula halophila Grun. (Clève, Syn. 1894, p. 109).

- Gendrei F. Hérib. (Pl. IX, fig. 31).

- major Ktz. (Diat. d’Aur, p. 82).

- nobilis Ehrb. (Diat. d'Auc., p. 80). 
Navicula rentricosa K̇tz. (V. H. Syn. pl. 12, fig. 19). - vulpina Ktz. (V. H. Syn. pl. 7, fig. 18).

Navicula lambertensis nov. sp. (Pl. XII, fig. 5). - De forme elliptique plus ou moins allongée; longueur 15 à $30 \mu$; raphé bien visible, à nodules terminaux petits et ronds, placés tout à fait contre les bords de la valve, les extrémités au nodule central non dilatées et assez distantes l'une de l'autre; aire hyaline axiale presque nulle, non dilatée autour du nodule médian; stries fines, mais nettes, courbes, la concavité tournée vers les extrémités de la valve, non distinctement granulées, au nombre de 10 en $10 \mu$ au milieu et de 15 en $10 \mu$ aux extrémités.

Diploneis Smithii Bréb. (A. Sch. Atl. pl. 12, fig. 49).

Stauroneis acuta WT. Sm. (Diat. d'Auv., p. 78).

- BaileyiEhrb. (Ehrb.S. und N. Amer.,p. 14)

Van-Heurckia rhomboides Bréb. var. amphipleuroides Grun. (Cl. Sym. 1894, p. 123). Bien couforme à la description et à la figure de Grunow.

Plourosigma attenuatum Ktz. (Diat. d'Aur., p. 122).

Cymbella affinis Ktz. (Diat. d'Aur., p. 66).

- aspera Ehrb. (Diat. d'Aue., p. 69).

Cymbella australica A. Sch. var. fossilis nov. (Pl. XII, fig. 14! - Diffère du trpe (A. Sch. Atl. pl. 10, fig. 34-35) par sa taille plus petite, longueur 70 à $85 \mu$, par ses stries plus courbées, parfois faiblement sinueuses, au nombre de $6{ }^{1} / 2$ à 7 en $10 \mu$ au milieu de la partie dorsale, de $7 \frac{1}{2}$ à 8 en $10 \mu$ à la partie ventrale, plus serrées aux extrémités, finement granulées ou divisées en travers. 
Cymbella Bruyanti F. Hérib. (Pl. XII, fig. 13). - De forme largement elliptique, à extrémités subitement et étroitement rostrées et capitées; longueur 60 à $70 \mu$, largeur 20 à $25 \mu$; raphé très légèrement et régulièrement arqué vers la face ventrale, ses extrémités at: nodule central très légèrement courbées vers le côté ventral, nodıles terminaux petits et ronds; aire hyaline axiale étroite, s'élargissant brusquement et fortement en losange autour du nodule central; stries radiantes, courbes, à convexité tournée rers la partie centrale de la valve, au nombre de 9 en $10 \mu$ an milieu de la partie dorsale, de 10 en $10 \mu$ au milieu de la partie ventrale, et plus serrées aux extrémités, où elles entourent les nodules terminaux.

Se distingue de notre Cymbella Laubyi par sa forme plus elliptique, par ses extrémités plus capitées, et par son aréa centrale plus grande et plus angulaire.

Ce Cymbella est dédié à M. Ch. Bruyant, professeur à l'Ecole de Médecine de Clermont, en souvenir des documents qu'il a eu l'amabilité de nous procurer.

Cymbella cymbiformis Ehrb. (Diat. d'Aur, p. 69).

- lanceolata Ehrb. (Diat. d'Aur., p. 68).

- leptoceras Ktz. (Diat. d'Auv., p. 66).

Encyonema prostratum Ralfs. (Diat. d'. Lur., p. 72). - rentricosum Ktz. (Diat. d'Aur., 1. 73.)

Gomphonema brasiliense Grun. var. fossilis nor. (Pl. XII, fig. 11). - Longueur 50 à $55 \mu$; stries au nombre de 15 à 16 en $10 \mu$, marginales, excepté à la partie supérieure, où elles arrivent progressivement presque jusqu'au raphé près du nodule terminal. - Diffère du type (V. H. Syn. pl. 25, fig. 17 par sa taille plus grande $(50$ à $55 \mu$ au lieu de 35$)$, par son contour plus 
accentué, et par la disposition des stries de la partie supérieure - Clève (Syn. 1894, p. 189) donne pour longueur de l'espèce 20 à $30 \mu$. - Le tṛpe n'a été trouyé rirant qu'au Brésil et ì Cuba.

Gomiphonema capitatum Ehrb. (Diat. d'Auv., p. 53). exiguum Ktz. (Diat. d'.1ux, p. 61)

Fragilaria Zrevistriata Grun. (Diat. d'Aur., p. 146). - - var. subcapitata Gr. (D.d'Auv.,p. 14i) - construens Grun. (Diat. d'Auv., p. 143).

Fragilaria construens Grun. var. binodis Grun. (T. H. Syn. pl. 45, fig. 24 et 25).

Ceratoneis antiqua nor. sp. (Pl. XII, fig. 17). - Espèce de petite taille; longueur 25 à $30 \mu$, courhure peu prononcée, extrémités légèrement capitées et largement arrondies; expansion ventrale légère, dépourvue de stries, présentant une légìre marque ou plissement en forme d'arc de cercle; stries rcbustes et écartées, 6 en $10 \mu$ au centre de la partie dorsale, un peu plus serrées aux extrémités, laissant au milieu de la valve une aréa lancéolée, assez large, et dilatée unilatéralement du côté ventral.

Eunotia pectinatis Rab. (Diat. d'Aur., p. 132).

Eunotia polyglyphis Grun. var. excisa no:. - Forme caractérisée par une légère excaration au milieu du bord rentral.

Epithemia Argus Ktz. (Diat. d'Auv., p. 127).

- - var. amplicepleala Grun. (1). 1Aur., p. 127).

- Ilyndmannii W. Sm. (Diat. d'Auv.,p.125).

- - var. perlonga nov. (Joursac, p. 28). 
Epithemia ocellata Ehrb. (Diat. d'Auv., p. 130).

- Sorex Ktz. (Diat. d'Aur., p. 126).

- turgila Ktz. (I)iat. d'Auc, p. 121).

- - var. gramu'ata Grun. (D. d'Aur., p. 125

- Zebra Ktz. forma minor nov. (D. i'tur., p. 129)

- - var. probos:idea Grun. (D.d'Aue.p. 128)

Rhopaloria gibba Ehrb. (Diut. d'. Lue., p. 126).

Grunouia Tabellaria Rab. (Diut. d'Aur., p. 166).

Cymat pleura elliptica IV. Sm. (Diat. d'Aur.,p. 160)

Opephora Murt,ji F. Hérib. (Diat. foss. d'Auz', p. 43).

Terpsinö̈ trifoliat a Clève (Der. Diat.de Fr., pl. 99, lig. 8

Ce doit être probablement cette Diatomée marine qui est indiquée à Cẹssac, daus les préparations Temfì̀re, sous le nom de Terpsinö̈ americana (Bail.).

Tetracyclus emarginatus IV. Sm. (Diat d'Aur., p. 158) - steila Ehrb. (Diat. foss. d'Aur., p. 17).

Tabellaria flucerulosa Kitz. (Diat. d'. Lur, p. 155).

Melosira arenaria Moor. (Diat. d'Aur., p. 186).

- bellicosa nov. sp. (Pl. XII, fig. 23).

- Boulayiuna M. Per. (Diat. d'Auc, p. 22?).

- lineoluta Grun. (Diat. d'Awe, p. 188).

- splecerica nov. sp. (Pl. XlI, fig. 24).

- temuis Grun. (Diut. d'Aue, p. 188).

Cyclotella antiqua $\mathbb{W}$. Sm. var. Vernierei F. Hérib. (Pl. XII, fig. 30). = Cyclotelía antiqua II. Sm. var. mirifica nov. F. Hérib. Disp. métle des Diat. d'Aluc., p. 47). - Très remarquable par la finesse de sil striation marginale et par le grand développement de sa partie centrale, qui est divisée en nombreux comparti- 
ments allongés, ne laissant au centre qu'une aréa très petite.

Cette belle Diatomée est dédiée à M. A. Vernière, ancien Président de l'Académie des Sciences, BellesLettres et Arts de Clermont-Ferrand, et auteur de plusieurs ourrages bien connus sur l'Histoire ancienne de l'Auvergne et du Telay.

Cyclutella comta Kitz. (Diat. d'Aur., p. 191).

Cyclotella comta Ktz. var. quadrinotata nov. (Pl. XII, fig. 28). - C'est la variété trinotata de Ceyssac, mais ayant quatre groupes de marques au lieu de trois.

Cyclotella comta Ktz. var. quinquenotata nor. (Pl. XII, fig. 29). - Même variété avec cinq groupes de marques .

Cyclotella Guignardi F. Hérib. (Pl. XII, fig. 29). - Espèce de grandeur moyenne; diamètre de 20 i $20 \mu$; circonférence finement striée, 12 à 13 stries en $10 \mu$ sur le bord; de 2 en 2 ou de 3 en 3 les stries sont renforcées, sur une certaine longueur, pour former les parois de grosses perles qui ne touchent pas les bords, et que l'on aperçoit en changeant la mise au point; le centre porte trois ou quatre surfaces triangulaires couvertes d'un sablé de points très fius, touchant la partie striée, le reste de la surface centrale étant lisse et sensiblement au même niveau.

Differe du Cyclotella antiqua $\mathrm{I}^{\circ}$. Sm., en ce que les surfaces triangulaires ponctuces, de la partie centrale, tonchent la circonférence striée, et qu'elles ne sont pas formées par des dénivellations de la face valvaire.

Nous dédions ce joli Cyclotella à II. Léon Guignard, Nembre de l'Institut. 
Cyclotella Temperei F. Hérib. var. inermis nor. - Se distingue du type du dépôt de la Bourbou?e, par l'absence des granules qui terminent les stries vers le bord de la valve.

Stephanodiscus Astrcex Krtz. (Diat. d'Aux., p. 193).

Les Diatomées caractéristiques sont: Cymbella lanccolata, Frayilaria construens, Epithemia Hyndmannii, likopalodia gibba, Melosira arenaria, bellicosa, spherica et Boulayiana, Cyclotella comta et ses rariétés.

D'après les préparations Tempère, les diatomistes attribuaient 18 espèces au dépôt de la Roche-Lambert; la liste ci-dessus comprend 64 espèces et variétés, parmi lesquelles 11 formes inédites. 



\section{DÉPOTS DE LARDECIE}

Les défôts de l'Ardèche, comme cenx de la HanteLoire, contiennent dans leur masse it Diatomécs, des empreintes de feuilles d'arbres, caractéristique des dépôts remaniés par les eaux; leurs florules diatomiques comprennent aussi, arec des espèces d'eau douce, des Diatomées marines et saumâtres, dont l'ensemble est tout i fait analogue aux florules des dépîts miocènes du ('antal, du Puy-de-Dôme et de la Haute-Loire.

Nous en concluons que ces argiles ì Diatomíes ne sont en réalité que des lambeaux restratifiés, provenant d'un dépôt initial formé dans un cratère-lac trachytique, dont les eanx profondes devaient être plus ou moins minéralisćes.

\section{DEPOT DE GOURGOURAS}

Gourgouras est situé sur les limites de l'Ardèche et de la Mante-Loire. Nous ne connaissons ce dépôt que par un échantillon reçu de M. l'abbé loulay; il en résulte que sa florule diatomique est encore incomplète. 
Toici les espèces observées:

Naricula major Ktz. (Diat. d'Aur., p. 82).

- hemiptera Ktz. var. angusta nov. (PI. II, fig. 20)

Rikopalodia giblerula Ehrb. (Diat. d'Aur., p. 129).

Opephoru Martyi F. Hérib. (Diat. foss. d'Aux., 1. 43).

Tetracyche emarginatus WV. Sm. (Diat. d'Aux., p. 158).

- Peragalli F. Hérib. (Pl. X, fig. 21).

Melosira Boulayiana M. Per. (Diut. d'Aux., p. 229).

- distans Ehrb. (That. d'Aur.,p. 185).

- granulata Ehrb. (Diat. d'Auc., p. 186).

- spiralis Ktz. (Diat. foss. d'Aur., p. 17).

- temuis Grun. (Diat. d'Aur., p. 18s).

Coscinocliscus Boulei H. Per. (Pl. XII, fig. 32).

Les cinq dernières espèces se trouvent en très grande abondance dans l'échantillon étudié.

L'ensemble de cette florule nous montre que Gourgouras est tout à fait analogue au Monastier et aux dépôts du Cantal. 


\section{DEPOT DE CHARAY}

Nous devons l'étude des dépôts remaniés de Charay, de Ranc et de Pourchères, à notre savant ami, M. le Commandant Maurice Perağ̉allo.

Les florules phanérogamiques des argiles à Diatomées de l'Ardèche ont été publiées par l'éminent doyen des Facultés catholiques de Lille, MI. l'abbé Boulay.

La liste suivante a été étiblie d'après l'examen de plusieurs échantillons, mais contenant ì peu près les mêmes Diatomées.

Cocconeis lineata Grun. var. euglypta Grun. (i.d Hur.p. to

- califormica Grun.(V.H. Syn.pl. 30, fig. 8-3)

- - var. subcontinua Grun. (Y. II. S:n. pl. 50 , fig. 10)

- Placentula Ehrb. (Diat. d'Aur.,p. 44).

Pieurosigma Brebissonii Grun. (V. H. Syn., pl 21, fig.6. .

Amplora lityca Ehrb. (Diat. d'Auv., p. 63).

Cymbella telética Kitz. (Diat. d'Aux., p. 72).

Encyonema lunula Grun. (Diat. d'Aur., p. 75).

Synedra Clna Ehrb. (Diat. d'Auv., p. 137).

- capitata Ehrb. (Diat. d'Aur., p. 139).

Epitlemia Hyndmannï W. Sm. (Diat.d'Aur', p. 125)

- Sore.c Ktz. (Diat. d'Aur., p. 126.

- Zebra hiz. var. proboscidea Gian. (D. d'Aur., p. 198)

- Argus Ktz. (Diat. d'Aut., p. 127). 
Hantzschia nov. sp.? - Longueur $50 \mu$; stries convergentes, très nettes, au nombre de 19 en $10 \mu$.

Melosira tenuissima Grun. (Diat. d'Auv., p. 188).

Cyclotella Comta Ktz. (Diat.d'Auv., p. 191). - Cette forme fossile ne porto pas l'épanouissement des stries du C'yclotella comtu vivant.

Cyclotella striata (Ktz.) var. ambigua Gr. (V. H. Syn., p. 92, fig. 12).

Au sujet de ce Cyclotella, caractéristique du dépôt par son abondance, MI. le Commandant Peragallo ajoute : Je n'ai pu identifier autrement cette espèce. M. Guinard, d'après une note du mémoire de M. l'abbé Boulay, l'a nommé Cyclotella minuta Ktz. Cette détermination ne me paraît pas admissible.

Il doit d'abord y aroir une erreur matérielle, car je ne connais pas de Cyclotella minuta décrit par Kutzing ni par aucun autre auteur. Kutzing décrit dans ses ouvrages (Bacillarien, p. 60 ; Species Algarum, p. 19), un C'yclotella minutula, qu'il dit exister dans le dépôt de Lunebourg; la seule description qu'il en donne est que son diamètre est inférieur ì $20 \mu$. Il y a bien dans le dépôt de Lunebourg un C'yclotella remplissant cette condition, mais il ne ressemble nullement ì celui de Charar, et je ne crois pas que l'on puisse admettre l'identité des deux formes.

Cyclotella nor.sp.? - Diametre $14 \mu$; stries au nombre de 10 en $10 \mu$, très nettes, dont une partie se prolonge jusque près du centre; quelques granules au centre; valye présentant une forte courbure vers un cercle situé à environ $1 / 3$ du diamètre à partir de la circonférence. - Analogue au Cyclotella Mtneghiniana, dont il n'est peut-être qu'une variété. 
Coscinodiscus exasperans Roth. - Tout ì fiit conforme à la fig. 9, pl. 58 de l'Attas de A. Schmidt.

Il est évident que cette liste ne nous donne qu'une idée très incomplète de la florule de Charar ; ce dépôt doit être étudié sur des échantillons plus nombreux et variés. 


\section{DÉPOT DE RANC}

L'examen d'une dizaine de préparations du dépôt de Ranc a donné le résultat suivant:

Rhoicosphenia currata Grun. (Diat. d'Auv., p. 51).

Achnanthes lanceolata Bréb. var. ovalis nov. - Diffère du type par sa forme ovale elliptique, et par l'absence de pseudo-stauros sur la valve inférieure.

Cocconeis lineata Grun. (Diat. d'. Iur., p. 44).

- scutellum Ehrb. (Nicr., pl. 15, fig. 56).

- - rar. minutissima nov. - Ne diffère de la forme type d'Ehrenberg que par sa taille trìs réduite.

Naricula rividis Kitz. (Diat. d'Aur., p. 83).

- appendiculata Ktz. (Diat. d'Aur., p. 95).

- scutelloides Grun. (1lg. Er. Fol. Diat., 1860, pl. 5 , fig. 15). - Se distingue de la fig. 34, pl. 6 de l'Atl. de A. Schmidt, par sa forme plus allongée; par l'aire hyaline axiale qui, au lieu d'être linéaire, s'élargit progressivement des extrémités au centre, où elle s'arrondit en s'élargissant encore autour du nodule médian; par deux ou trois points intercalés vers le bord de la valve entre les lignes rayonnantes.

Navicula gastroides Greg. (M. J. 1855, pl. 4, fig. 17).

Diploneis elliptica Ktz. (Diat. d'Aur., p. 101).

Pleurosigma acuminatum Grun. (Diat.d'Auv., p.122) 
Cymbella helvetica $I$. Sm. var. nov.? - Frustule renflé au centre; raphé droit, avec les extrémités centrales infléchies vers la partie dorsale; nodules terminaux petits, ronds, touchant la face dorsale; valve à centre gibbeux, à extrémités légèrement coniques et largement arrondies; stries nettes, convergentes, laissant entre elles et le raphé un espace lisse et bien défini, très faiblement dilaté autour du nodule médian; on compte 11 stries en $10 \mu$ à la partie dorsale, et 14 en $10 \mu$ à la partie ventrale.

Cymbella delicatula Ktz. var. nov.? - Petite forme lyyaline, analogue à la figure 55, pl. 71 de l'Atl. de A. Schmidt.

Cymbella gibba Ehrb. var. nov.? - Ressemble à la figure 27, pl. 10 de l'Atl. de A. Schmidt, mais elle en diffère par les stries, qui viennent se terminer du côté du raphé par un renflement, délimitant une aréa lisse autour du raphé, sans élargissement sensible autour du nodule médian, de sorte que la partie la plus visible de la strie est celle qui est près du raphé. La longueur du frustule varie de 32 à $45 \mu$.

Gomphonema abbreviatum Ktz. var. acuminata noc. - Forme analogue à la figure 26, pl. 23 du Sym. de Ian-Heurck, mais avec la striation des figures 16 et 17 de la pl. 25 du même auteur; valve à extrémités plus acuminées; longueur $25 \%$; stries au nombre de 16 en $10 \mu$.

Synedra Ulna Ehrb. (Diat.d'Auv., p. 137).

Fragilaria brevistriata Grun. (Diat. d'Aur., p. 146).

- lapponica Grun. (Diat. foss. d'Aur., p. 21)

- virescens Ralfs. (Diat. d'Aur., p. 147). 
Fragilaria costata II. Per. nor. sp. - Espèce très petite; longueur 8 à $12 \mu$; face val raire bacillaire, à terminaisons arrondies ou ovales allongées; 8 ì 9 perles marginales en $10 \mu$; de chaque perle part une côte qui va en s'évanouissant jusqu'au centre de la valve; les côtes des deux flancs de la valre sont alternes; la face connective est rectangulaire, cannelée de chaque côté par une série de côtes qui dépassent la surface valvaire pour former les perles. - La striation de la valve ressemble à celle d'un Surirolla, mais la face connective est bien celle d'un Fragilaria.

Eunotia Arcus Ehrb. (Diat. d'Aur., p. 130).

Epithemix Sorex Ktz. (Diat. d'Aur., p. 126).

- Zebra Ktz. (Diat. d'Aur., p. 127).

- - var. proboscidea (irun. (D.d'Aur.,p.128)

- Testermannii Ktz. (Diat. d'Aur., p. 125).

- Ilyndmannï W. Sm. (D. d'Aur., p. 125).

- ocellata Ehrb. (Diat. d'Aur, p. 130).

Epithemia fenestrata 11. Per, nor. sp. Frustule petit, trapu; face ralvaire en demi-lune, dont les extrémités arrondies sont quelquefois un peu prolongées; la valve comprend deux parties bien distinctes: la partie dorsale, formant une bande parallèle au dos et comprenant les extrémités, est simplement striée; les stries sont convergentes, et au nombre de 16 à $18 \mathrm{en}$ $10 \mu$; la partie centrale est munie de côtes ( 1 à 5 en $10 \mu$ ) dans le prolongement des stries; les stries de la bande dorsale se continuent entre les côtes, mais elles sont beaucoup moins marquées; la ligne ventrale est droite ou légèrement concave; la face ventrale est ovale ou orbiculaire; elle montre ordinairement une ligne de suture axiale, et les côtes des valves qui lui sont presque perpendiculaires. 
II. le Commandant M. Peragallo a trouvé des Epithemia tout à fait analogues en Algérie, dans des sources thermales fortement minéralisées.

Rhopalodia gibberula Ehrb. (Diat. d'Auv.,p. 129). - - - var.producta Grun. (D.d'Aur.,p.129

Grunovia Tabellaria Grun. (Diat. d'Aur., p. 166). - Le Cirunorcia de Ranc differe du type de Grunow par ses extrémités simplement coniques, un peu prolongées et non capitées.

Surirella marginulata II. Per. nov. sp. Longueur de la valve 30 à $40 \mu$, de forme ovale très allongée, presque bacillaire, ̀̀ extrémités arrondies, trìs peu différentes l'une de l'autre; côtes marginales en forme de dents, au nombre de 10 en $10 \mu$.

Melosira naritis W. Sm. (Diat. d'Aur., p. 185).

- undulata Ktz. (Diat. foss. d'Aur., p. 22).

- Boulayiana MI. Per. (Diat. d'Auv., p. 229).

Cyclotella stelligera Cl. et Gr. (V. II. Sym. pl. 94, lig. 22 a 26).

Le dépôt de Ranc contient aussi un mélange de Diatomées d'eau douce et saumâtres, caractéristique d'une formation miocène dans un cratère-lac trachytique. 


\section{DÉPOT DE POURCHERES}

Les échantillons étudiés du dépôt de Pourchères, prìs de Privas, se présentent sous la forme d'une matière désagrégće, plus ou moins pulvérulente, s'écrasant facilement sous les doigts et légèrement îpre an toucher.

Leur couleur est d'un gris pâle plus ou moins blanc.

La masse est presque exclusirement formée de Diatomées et ne fait pas efferrescence sous l'action des acides.

Tous les frustules, même ceux de petite dimension, sont fragmentés et désorganisés, mais les points et les stries restent très nets sur les fragments, ce qui tendrait à faire croire que les agents qui ont produit le fractionnement étaient plutôt des agents phṛsiques que chimiques; on ne troure pas un seul frustule un pen grand intact; souvent les parties striées sont séparées des parties lisses; c'est ainsi, par exemple, que le $D i$ p’oneis elliptica, qui est fréquent dans le dépôt, se trouve presque toujours dépourvu du nodule central.

Les espèces sont assez nombreuses, mais leur état de fragmentation en rend la détermination très difficile.

Les Diatomées observées dans les échantillons examinés sont :

Cocconeis lineata Grun. (Diat. d'Aur., p. 44).

Navicula menisculus Schum. (Diat. d'Aur., p. 98).

- ractiosa Ktz. (Diat. d'Aux., p. 99).

- major Ktz. (Diat. d'Aur, p. \&2). 
Diploneis elliptica Ktz. (Diat. d'_lur., p. 10t).

Cymbella helvetica Kitz. (Diat. d'Aur., p. 72)

- parea IV. Sm. Diat. d'Aur., p. 70!.

- maculata Kitz. (Diat. d'Aur., p. 71).

- cistula Hempr. (Diat. d'Aur., p. 70).

- aspera Ehrb. (Dict. d'Aur., p. 69).

- alpina Crun. (A.Sch. Atl., pl. 71, fig. 45).

Cymbella arctica Lag. var.? - Ressemble à la figure 25, pl. 71 de l'Atlas de 1 . Schmidt, mais il est plus large, moins courbé et les extrémités sont moins tronquées; longueur de la ralve $85, u$; stries au nombre de $S$ en $10 \mu$, atteignant de près la ligne médiane, celles de la face ventrale non interrompues vers le nodule médian, et présentant à cet endroit un renflement très marqué.

Un autre Cymbella du même dépôt se rapproche, comme forme, dn Cymbella leungarica Grun., mais il en diffère par les stries plus serrées (11 en $10 \mu$ au lieu de 8 en $10 \mu$; par la présence, an milieu de la valve, d'un point brillant, analogue à celui de certains Gomphonema; ce point est accompagné de deux macules, et se trouve situé du côté de la face dorsale; il est excentrique par rapport au raphé, dont les deux extrénités convergent vers lui; les stries s'arrêtent à une certaine distance du raphé; celles quii sont au centre de la valre sont encore plus courtes, et forment autour du nodule une aréa notable; longueur $57 \mu ; 11$ stries en $10 \mu$ à la face dorsale et 12 en $10 \mu$ à la face ventrale.

Encyonema lunula Grun. (Diat. d'Auv., p. 75).

Encyonema nou. sp.? - Arec l'Encyonema lunu'c Grun., on trouve une autre forme dont les caracteres ne 
conviennent à aucun Encyonema connu, et qui mériterait peut-être d'être distinguée comme espèce. - Voici ses principaux caractères :

Longueur $34 \mu$, largeur $9 \mu$, arec 7 stries en $10 \mu$; raphé droit; face ventrale étroite, légèrement renflée au centre; face dorsale régulièrement courbée; extrémités arrondies, faiblement proéminentes; surface valvaire couverte de stries bien définies, plus grosses au centre qu'aux extrémités, et sur la face dorsale que sur la face ventrale; sur les bords de la valve, elles sont terminées par un grauule.

Gomphonema subclaratum Grun. (Diat.d'Aux.,p.555)

- insigne Grun. (Diat. d'Aux., p. 59).

- montanum Schum. (D. d'Aur., p. 55).

Gomphonema nor: sp.? - Forme légèrement anguleuse, à angles arrondis; raphé à extrémités centrales inclinées vers un granule concentrique trìs visible; longueur de la valve 40 à $45 \mu$, largeur 9 à $10 \mu$. A un grossissement de 1500 diamètıes, les stries ont l'aspect de petites côtes (ou sillons) très étroites, espacées, tout à fait lisses.

Epithemia Westermannii Ktz. (Diat. d'Aur.,p. 125). - Hyndmanmii W. Sm. (D. d'Aur., p. 125.

\section{Epithemia Hyndmannii var. interrupta} $n o v$. - Cette variété est caractérisée par la particularité suivante :

La cloison longitudinale, arrondie au sommet, est formée de deux lames; la ponctuation de la surface de la valve s'arrête à ces lames et fait défaut sur la cloison; les côtes transversales changent brusquement de direc- 
tion pour traverser la cloison normalement, et reprennent ensuite leur direction primitire.

Eunotia polyglypleis Grun. (Diat. d'Awe., p. 134).

-- - var. penteglypleis Grun. - Analogue à la figure 33, pl. $3 \pm$ du Synopsis de Van-Heurck, mais ayant 5 dents.

Grunowia Tabellaria Rah. var. major not. - Longueur 35 a a vec 6 côtes en $10 \mu$; stries très nettes, au nombre de 18 en $10 \mu$.

Surivella turgida W. Sm. (Diat. d'Aur., p. 176).

Me'osira undulata Ktz. (Liat. foss. d'A Lue, p. 22). - arenaria Hoor. Diat. d'Aue., p. 186).

Melosira arenaria var. lævis $n o x$. - Differe du type en ce que les stries sont simplement marginales et courtes; par suite le reste de la surface valvaire est tout à fait lisse.

Melosira granulata Ehrb. (Diat. d'Aur., p. 186).

- - var.currataGr.(T.H.Syn.,pl.87, fig. 18-19)

Les espèces obserrées le plus souvent sont: Diploneis elliptica, Cymbella aspera et helvetica, Epithemia Westermannï et Hyndmannii, Melosira unduluta et gramulata, l'un et l'autre très communs.

Les argiles diatomifères de la Haute-Loire et de l'Ardèche nous ont livré 43 Diatomées que nous n'avons pas constatées daus les dépôts miocènes du Puy-deDôme et du Cantal.

Si nous ajoutons ce nombre aux 908 espèces et variétés trourées en Auvergue, on voit que la flore diato- 
mique du Plateau Central comprend aujourd'hui plus de 950 Diatomées très distinctes.

Les diatomistes et les paléobotanistes trouveront dans le sarant mémoire de MI. l'abbé Boulay (Bull. Soc. bot. de Fr., t. xxxiv, séance du 27 ma 1887), tous les détails concernant la topographie, la stratigraphie et les florules phanérogamiques des dépôts de l'Ardèche.

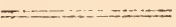




\section{OBSERVATIONS}

SUR LES DÉPOTS TERTIAIRES A DIATOMÉES

DU PLATEAU CENTRAL

\section{I}

Nous sarons maintenant que les argiles à Diatomées du Cantal proviennent du dépêt initial de ChambeuilFraisse-Bas, et que leur formation miocène a dû s'effectuer dans un cratère-Jac profond et d'une grande étendue.

Démentelée par les poussées rolcaniques, la masse diatomifère, englobée dans les projections andésitiques, a été entrainée à des distances variables du point où s'est ouvert le cratère.

Parmi ces lambeaux de projection, il en est, comme Joursac et Andelat, qui oṇt été repris par les eaux, ì l'époque tout il fait supérieure du Miocène, et se sont restratifiés dans les dépressions préexistantes où nous les trouvons aujourd'hui, avec les empreintes de feuilles d'arbres, charriées pêle-mêle par le courant boueux diatomifère, ou apportées par les courants aériers dans la fosse où s'effectuxit la sédimentation.

Les lambeaux non remaniés par les eaux, comme Auxillac, Celles, Moissac, etc., ne contiennent pas d'empreintes de feuilles d'arbres, parce qu'elles n'ont pu se fossiliser au cours de la formation miocène du dépôt initial. A Celles nous indiquons bien, dans notre pre- 
mier mémoire, une empreinte de Fagus, mais ce fragment, très incomplet, trouvé à la surface d'un échantillon englobant un caillou roulé, le tout intercalé dans la formation morainique, uous paraît aujourd hui tout à fait accidentel et sans raleur docunentaire.

Ainsi donc, les gisements à Diatomćes du Cantal, ì l'exception de Joursac et d'Andelat, sont des lambeaux non remaniés par les eaux, arrachés en blocs du dépôt initial par les explosions andésitiques et englobés dans leurs produits de projection. - Ce fait est d'ailleurs très fréquent pour d'autres roches préandésitiques: MII. Boule et Marty ont découvert, dans la vallée de Brezons, à plus de 20 kilomètres du cratère, un lambeau de calcaire oligocène, exploité pour la fabrication de la chaux, et parfaitement englobé dans la brèche andésitique, dont les éruptions, après l'avoir arraché de la région du cratère, c'est-ì-dire près de Laveissière, oì le calcaire oligocène est encore utilisé pour la fabrication de la chaux, l'avaient charrié, à l'état d'enclare, jusque dans la vallée de Brezons. Notre interprétation des lambeaux non remaniés par les eaux est done conforme à d'autres faits d'observation.

En notant, sur la carte du Cantal, la position des dépôts ì Diatomées, on constate qu'ils sont tous situés dans un même secteur du grand volcan; en dehors de ce secteur, nous n'arons observé nulle trace d'argiles diatomilères.

Les dépôts tertiaires du Puy-de-Dôme, c'est-ì-dire Varennes, La Bourboule, Perrier et Saint-Saturnin, appartiennent à la catégorie des lambeaux remaniés par les eaux; mais le remaniement s'est effectué à des 
époques très différentes: si, pour Varennes, Perrier et La Bourboule, il a eu lieu vers le Pliocène moyen, le dépôt de Saint-Saturnin a ćté certainement restratifié en pleine période quaternaire, c'est-à-dire à l'époque des ćruptions rolcaniques de la chaîne des Monts Dômes; sa florule phanérogamique est en effet franchement quaternaire.

$A$ la suite de recherches concernant l'origine des argiles a Diatomées du Puy-de-Dôme, nous avons acquis la conviction que le dépôt initial d'où elles proviennent ne peut être que celui des Egravats, situé entre la Grande-Cascade du Mont-Dore et la base du Sancy, à 1350 mètres d'altitude, dans la région où devait s'ouvrir l'un des cratères des volcans trachytiques du massif montdorien.

De même qu'ì Chambeuil-Fraisse-Bas, le dépôt des Egravats est intercalé entre des projections trachytiques, des argiles ligniteuses et des formations andésitiques; en outre, la masse diatomifère, très argileuse, ne contient pas d'empreintes de feuilles d'arbres, et présente tous les caractères d'un dépôt en place.

Il est à noter que les argiles à Diatomées des Egravats se trouvent au sommet de la vallée de la Bourboule, et à peu de distance de la vallée de Chaudefour, au bout de laquelle est situé le dépôt de Tarennes. Quant au dépôt de Perrier, il ne se présente que par lambeaux peu volumineux disséminés dans des tufs ponceux et des conglomérats d'origine glaciaire ou fluviatile, à éléments plus ou moins grossiers; ses florules diatomique et phanérogamique ne sont encore que somrnairement connues.

Les Diatomées des Egravats se retrouvent à $\mathrm{V}_{\mathrm{i}}$ rennes; mais, de même que la florule de ChambeuilFraisse-Bas est très pauvre, comparée à celle de Jour- 
sac, la florule des Egravats est peu variée, en comparaison de celle de Varennes, ainsi que le montre la liste publiée dans notre premier mémoire, p. 26 et 27 .

L'examen récent de deux nouveaux échantillons nous a permis d'ajouter six espèces à celles déjà connues, ce sont: Vacicula Esox, peregrina et Smithii, Epithemiu turgita et Melosira varennarum, représenté par un fragment comprenant les trois quarts de la vue valvaire, et Coscinodiscus lyggmous, forme intermédiaire entre nos variétés micropunctato et crassipunctata.

Le nombre des Diatomées des Egravats s'élève actuellement à une trentaine, parmi lesquelles plusieurs sont caractéristiques des lambeaux de Varennes, de la Bourboule et de Perrier.

D'après ces faits lien constatés, nous concluons que les argiles diatomitères du Puy-de-Dôme, excepté celles de Saint-Saturnin, proyiennent du dépôt initial du ravin des Fgravats, et, comme dans le Cantal, ces masses i Diatomées d'eau douce, marines et saumâtres, se sont formées dans des cratères-lacs trachytiques, dont les eaux devaient être plus ou moins minéralisées.

Nous laissons aux paléobotanistes le soin de fixer l'âge précis de la florule phanérogamique, postérieure à la formation miocène du dépôt initial.

Ainsi que nous l'arons dit ailleurs, pour tous les dépôts remaniés, contenant des empreintes de feuilles, les deax florules sont absolument indépendantes quant à leur âge géologique.

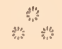

Pour les dépôts de la Hante-Loire et de l'Ardèche, nous ne sommes pas assez au courant de la géologie de 
la région pour pouroir fixer exactement le dépôt initial.

Toutes les argiles à Diatomées de ces deux départements contiennent des empreintes de feuilles, preuve évidente qu'elles ont été restratifiées par les eaux.

Il est très probable que les lacs où vivaient les Diatomées étaient situés dans les cratères du trachỵte inférieur miocène du Mezenc.

Ces lacs cratériels, dont les eaux deraient être excessivement farorables à la multiplication des Diatomées, vidés par les explosions volcaniques concomitantes de nouvelles éruptions, leurs dépôts à̀ Diatomées ont été entrainés vers la périphérie du volcan, pour se restratifier à sa base, pêle-mêle avec les feuilles et autres objets rencontrés sur leur route, comme pour les dépôts miocènes du Cantal et du Puy-de-Dôme.

Ces restratifications ont dî s'opérer, soit dans des lacs plus ou moins profonds situés au pied du rolcan, ainsi que l'indiquent la schistosité du sédiment et les poissons qu'il renferme, soit dans une dépression du sol.

D'après la faune et la flore phanérogamique, le remaniement des dépôts de l'Ardèche situés dans le bassin du Rihône, daterait, comme pour Joursac, du Miocène supérieur ou Pontien.

La restratification des dépôts de la Haute-Loire situés dans le bassin de la Loire, en particulier ceux de Ceyssac et de la Roche-Lambert, se serait effectuée i l'époque du Pliocène moren ou Astien.

Mais l'analogie de leurs florules diatoniques arec celles des dépôts du Virarais, le fait qu'on y trouve ì l'état de cailloux roulés toutes les roches du Mezenc, sont pour nous l'indice, presque la preuve, que le dépôt initial est le même que pour ceux de l'Ardèche. C'est la continuation, dans le Pliocène, d'un phénomène de rema- 
niement des lambeaux pontiens infra-rolcaniques du Mezenc, dont les atterrissements de Gourgouras, du Monastier, etc., marquent le premier stade. - Il se pourrait aussi que les dépôts pliocènes, au lieu de provenir des dépôts initiaux du Pontien, fussent un second remaniement du dépôt, également pontien, mais déjà remanié, du Monastier.

En résumé, il a existé dans le Cantal, les Monts Dures et le Mezenc, à la fin du Miocène, de vastes dépôts à Diatomées, dont la formation a été extraordinairement farorisée par des circonstances encore assez olscures, mais probablement liées aux sources thermales et aux cratères-lacs trachytiques, comme cela se produit actuellement à Java, oì la modalité volcanique a beaucoup de rapport arec celle du Cantal ${ }^{(1)}$.

Toujours dans le Miocène supérieur, ces argiles diatomilères ont été disloquées par les mouvements sismiques corrélatifs des éruptions, soumises aux érosions consécutives des grandes pluies qui tombent sur les flancs des volcans dans leurs phases de paroxysme, et restratifiées dans les dépressions situées au pied de ces volcans.

(1) En notant, sur la carte de l'Europe centrale, la distribution géographique des dupôts a Diatomes, on roit qüils sont tous localis is dans les régions voleaniques: cette constatation se troure même virifiée pour les dépôts extra-européens.

Pour la France, en particulier, nous nen connaissons pas dans les contrées a sol stable, comme les monts cristallins du Forez, les Tosges, ete.

De plus, au cours de nos etudes sur les Diatomées d'Auvergne, nous avons acquis la certitude que la multiplication de ces microphytes est tris active dans tous les cratires-lacs de la région volcanique, tandis qu'elle est très lente dans les lacs occupant une dépression du sol primitif, comme le lac de la Crégut (Cantal).

La formation des dépôts diatomifères, ne serait-elle pas, comme les sources thermales, une modiliti du vulcanisn e?... 
Dans le bassin du Puy, ces phénomènes de remaniement, soit immédiat, soit médiat, des argiles pontiennes à Diatomées, ont eu une recrudescence au milieu du Pliocène.

Nos argiles à Diatomées nous ont livré, avec une série nombreuse d'espèces d'eau douce, des espèces marines et saumâtres. Pour nous, il est de toute éridence que ces deux dernières catégories de Diatomées ne pouvaient vivre et se développer dans des lacs d'eau douce ${ }^{(1)}$. Pour expliquer leur présence dans les dépôts tertiaires, nous avions songé à leur attribuer une origine lagunaire oligocène. L'hypothèse était d'autant plus séduisante qu'une formation de cette nature, d'ailleurs unique, se trouve dans le Cantal; ce sont des marnes calcaires intercalées entre deux niyeaux d'eau douce; mais nulle part ces marnes n'ont montré de dépôts diatomifères; c'est pourquoi nous avons dû abandonner cette hypothèse et diriger ailleurs nos investigations.

Voici la solution que nous proposons :

Il existe actuellement, autour des nombreuses sources salines d'Auvergne, une florule composée de plantes

(I) Nous n'avoris, en effet, jamais constaté de Diatomées marines dans les dépôts quaternaires du Puy-de-Dôme, dont la formation s'est opérée dans des lacs d'eau douce. 
exclusivement maritimes, telles que: Spergularia marginata et salina, Trifolium maritimum, Taraxacum leptosephalum, Glaux maritima, Triglochin maritimum, Glyceria distans, Polypogon monspeliense, Chara crinita var. brevispina, Pottia IIeimii, etc.

Nous n'y trouvons pas, il est vrai, de Diatomées franchement marines, ni même de formes saumâtres exclusives.

L'absence d'espèces marines tient sans doute à ce que les Diatomées, plus exigeantes que les plantes supérieures, ne trouvent pas, dans nos eaux minérales actuelles, le degré de salure dont elles ont besoin pour vivre et se multiplier.

Mais de ce que ces sources sont aujourd'hui relative. ment peu minéralisées, il ne faut pas conclure qu'il en fut toujours ainsi.

Les sources minérales n'étant qu'une modalité du vulcanisme, il est normal de supposer qu'à l'époque où les volcans du Plateau Central étaient en pleine activité, c'est-ì-dire au moment où se déposaient, en alternance avec des projections trachytiques, les argiles ì Diatomées de Chambeuil-Fraisse-Bas, des Egravats et du Nezenc, ces sources devaient contenir plus de sels en dissolution qu'elles n'en contiennent aujourd'hui, où les foyers éruptifs de ces régions calcinćes sont depuis si long temps éteints.

On sait d'ailleurs que les éruptions, surtout les éruptions trachytiques, sont toujours accompagnées de projections boneuses, liquides ou gazeuses, de nature très variée, au point de vue de leurs éléments minéraux.

D'après M. de Lapparent, le chlorure de sodium apparait comme l'élément initial des produits des fumerolles, et c'est par des émanations d'hydrocarbures, ac- 
compagnées d'eau salée que s'est annoncée l'éruption survenue en mai 1879 à l'Etna(1).

En général, ajoute Poulett Scrope, après la cessation d'un paroxisme rolcanique, plusieurs fumerolles, ou émanations de vapeurs, s'échappent des courants de lave qui se sont alors produits, aussi bien que du fond du cratère. Ces vapeurs contiennent ordinairement une certaine quantité d'acides minéraux, et, à mesure que la lave se refroidit, elles déposent des incrustations salines à l'orifice des fumerolles. I.es acides sont : l'acide chlorhydrique, l'acide sulfurique, l'hydrogène sulfuré, etc., ou leurs composés, surtont les chlorures de sodium, de potassium, d'ammonium et de fer.

Le même auteur mentionne que sur les flancs du mont Idjeng, volcan javanais qui présente rers l'E. un cratère-lac, jaillissent de nombreuses sources salées (2); c'est dans les dépôts des eaux salées de Java que se trouve abondamment Stanroneis jacanica Grun., l'analogue de notre Stauroneis jacanica Gr. var. arvernense (Pl. XI, fig. S) des argiles diatomitères de Moissac, ainsi que le Stictodiscus pulchellus Grum., Diatomée jaranaise du dépôt de Ceyssac.

On pourrait multiplier ces citatious. Elles suffisent pour montrer que dans beaucoup de volcans plus ou moins acides, en particulier dans ceux de Java, que II. Boule compare si justement avec le volcan du Cantal, il existe des cratères-lacs et des sources salines fortement minéralisées, qui font partie des manifestations de l'activité volcanique; l'ailleurs, nous constaterons plus loin que la flore diatomique de nos argiles miocènes est très analogue à la flore actuelle de Jara.

(1) Ie Lapparent: Traité de Géol, p. 416 et 414.

(2) Poulett Scrope : Les Volcans, p. 26 et 479. 
Et ce n'est pas, croyons-nous, forcer les analogies que de supposer qu'il en fut de même dans le Plateau Ceutral, pendant la période Miocène, au cours de laquelle se formèrent les dépôts à Diatomées.

Cette interprétation si rationnelle rend compte de l'existence, daus les dépôts du Cantal, comme dans ceux du Puy-de-Dôme, de la Haute-Loire et de l'Ardèche, de Diatomées marines et saumâtres, ayant vécu dans des cratères-lacs trachytiques, alimentés par les eaux salines de sources abondantes et plus minéralisées qu'elles ne le sont à notre époque géologique.

Telle est notre manière de comprendre la formation et le remaniement des argiles diatomifères du Plateau Central, et d'expliquer la présence des Diatomées marines et saumâtres qu'elles nous ont livrées.

A l'appui des considérations précédentes, voici la florule des espèces marines et saumâtres observées dans nos argiles tertiaires à Diatomées.

Abrév. - $\mathrm{C}=$ Cantal, $\mathrm{P}=$ Puy-lle-Lôme, $\mathrm{II}=$ Haute-Loire,

$\Lambda=$ Ardèche.

\section{ESPĖCES MARINES}

Achnanthes subsessilis Ktz. (P.).

Cocconeis scutellum Ehrb. (A.).

- speciosa F. IIérib. (C. P.).

- trilineatus F. Hérib. et II. Per. (C. P.).

Coscinodiscus Boulei H. Per. (H. A.).

- exasperans Roth. (P. A).

radiatus Grun. (P. A.).

Cyclotella striata Ktz. var. ambigua Grun. (A.). 
Diploneis Smithii Bréb. (C. P. H.). Gomphonema arcticum Grun. (C.). - exiguum Ktz. (C.). - Kamtschaticum Greg. (P.).

Goniothecium odontella Ehrb. (H.). Melosira Sol Ehrb. (C.).

Naricula cellesensis 5 . Hérib. (C.). Pleurosigma Brelissonï Grun. (A.). Stauroneis parrula Grun. (C.).

- jaranica Grun. var. areernense nov. (C.. Stephanodiscus Astrea Ktz. (C. P. H.).

- - var. minutula Grun. (P.).

Stictodiscus pulchellus Clève. (H.).

Synedra lancettula Grun. (C.).

Terpsinoe trifoliata Grun. (H.).

\section{ESPECES SAUMATRES}

Achnanthes lanceolata Gr. var. elliptica Cl. (C. H.). Cocconeis Placentula Ehrb. var. minor nov. (C.). Coscinodiscus decipiens Grun. (H.).

- clambonis F. Hérib. et M. Per. (P.).

- dispar MI. Per. et F. Hérib. (C. P.).

- pygmaus F. Hérib. et M. P. (C. P. H.).

Cyclotella Meneghiniana Ktz. (C. P. H.).

Cymatopleura hybernica IV. Sm. (P.).

Cymbella leptoceras Kitz. var. curtc V. H. (C.).

$$
\begin{aligned}
& \text { - parra W. Sm. (I.). } \\
& \text { - pusilla Grun. (C. P.). }
\end{aligned}
$$

Diploneis elliptica Ktz. var. minutissima Gr. (C.).

- Pagesi F. Hérib. (C.).

Epithemia fenestrata MI. Per. (A.).

-- Hyndmannii W. Sm. var. curta F.H. (P.) 
Eunotia polyglyphis Grun. (C. P. H. A).

Fragilaria Urevistriata Gr. var. Mormorum G. (C. H.) Gomphonema semiapertum Gr. var.tergestina G.(C.P.) brasiliense Grun. var. fossilis nor.(H.). Ilantzschia amphioxys Gr. var. intermedia Grun. (C.) Saricula amphibola Cl. (C. H.).

- - var. perrevi F. Hérib. (P. H.).

- aponina Ktz. (P.).

- arerna F. Hérib. (C. P. H.).

- Berriati F. Hérib. (C. H.).

- Braunï Grun. (P.).

- halophila Grun. (H.).

- Hitcheockiii Ehrb. (C.).

- Malinvandi F. Hérib. (C).

- peregrina Heib. (C. P.).

- Porrecta Ehrb. (P.).

- Reinhardtii Grun. (C. H)..

- Renauldi F. Hérib. (C.).

- rostellata Ktz. (C. P.).

-- sculpta Ehrb. (C.).

- slesicensis Grun. (C.).

- transrersa A. Sch.

Melosira tenuissima Grun. (C. P. H. A.).

Nitzschia Brebissonii Wr. Sm. (C.).

- tubicola Grun. (P.).

likopalodia gibba Ehrb. var. ventricosa Grun. (C.H.).

- gibberula Ktz. var. producta Gr. (C. P.). Surivella turgida W. Sm. (P.).

Siynedra closterioides Gr. var. fossilis F. Hérib. (C.). - delicatissima W. Sm.

- rumpens Ktz. (C. H.).

Telles sont les espèces admises par la généralité des diaomistes comme étant respectivement marines et saumâtres exclusives. 
Nous aurions pu ajouter à la liste des Diatomées saumâtres un assez grand nombre d'espèces préférentes, c'est-à-dire des Diatomées qui vivent de préférence dans les eaux saumâtres, mais que l'on troure aussi, plus ou moins fréquemment, dans les eaux douces; nous arons pensé que nos deux listes, ne comprenant que des espèces exclusives, suffisaient largement pour montrer que les argiles miocènes à Diatomées du Plateau Central, n'ont pu se déposer que dans des eaux plus ou moins minéralisées.

Les Diatomées marines et saumâtres du Puy de Mur (Puy-deDôme) ne figurent pas dans les deux listes précédentes, parce que la formation lagunaire de ce curieux dépôt étant un fait bien acquis, son origine est tout à fait différente de celle des argiles à Diatomées de l'époque Miocènè.

Nous nous sommes abstenu également d'inscrire la plupart des espèces inédites, parmi lesquelles il en est pourtant une vingtaine dont l'aspect général et la striation caractérisent des formes que nous n'ayons jamais constatées dans les dépôts d'eau douce; mais ne possédant pas de documents à leur sujet, nous avons dî nous borner à n'en mentionner qu'un très petit nombre dont l'origine saumâtre est de toute évidence. 


\section{DISTRIBLTION GÉOGRAPHIQUE}

DES DIATOMÉES TERTIARES DU I'LATEAU CENTRAL

DIATOMÉES DE YOS ARGILES MOCÈ̃ES COMPARÉES A CELLES DE QUELQZEES DÉPOTS DU TORD DE L'EURORE

Une comparaison de ce genre ne devrait porter que sur des dépôts bien datés; malheureusement nous ne sommes pas absolument fixé sur l'âge précis de ceux du Nord de l'Europe dont nous connaissons les florules diatomiques.

D'après II. le Commandant M. Peragallo, ils appartiendraient au Quaternaire, mais nous n'avons pu nous renseigner exactement.

Malgré cette incertitude, nous croyons qu il n'est pas sans intérêt de rechercher les Diatomées qui, depuis l'époque Miocène, ont émigré dans les régions boréales.

Les dépôts comparés sont :

Ordie $($ Ecosse $)=$ Ord. - Sirexe $($ Ecosse $)=$ Sh. - RyssbyCalmar (Suede) = Ryss. - Stavajger (Norvège) $=$ Stav.

Les Diatomées commune à ces dépôts et à nos argiles miocènes sont les suivantes:

Amphora oralis Ktz. (Sh. Ryss.).

-. Pediculus Grun. (Ryss.).

Campy'opus costatus WT. Sm. (Ryss.). 
Cocconeis Placentula Ehrb. (Ryss.).

Cyclotella antiqua IV. Sm. (Sh.).

- Kutzingiana Chauv. (Stav.).

- comta Ktz. (Ryss.).

Cymatopleura elliptica IV. Sm. (Ryss.).

- Solea Breb. (Sh. Riyss.)

- hybernica IT. Sm. (Ord.).

Cymbella cistula Hempr. (Ord. Ryss. Star.).

- cuspidata Ktz. (Sh.).

- cymbiformis Ehrb. (Ord. Sh.).

- Ehrenbergü Greg. (Ord. Sh. Ryss.).

- gastroides Kitz. (Sh. Ryss.).

- helietica Ktz. (Ryss. Star.).

- lanceolata Ehrb. (Ord. Ryss.).

- leptoceras Ktz. (Ryss.).

- parra WV.Sin. (Sh.).

Diploneis elliptica Ktz. (Ryss.).

Encyonema ccespitosum Ktz. (Sh.).

- turgidum Grun. (Ord. Sh.).

- rentricosum Kitz. (Ord).

Epithemia Argus Ktz. Ord. Sh. Ryss. Stav.).

- IIyndmannï IV. Sm. (Sh. Ryss.).

- Sorex Ktz. (Ord. Sh. Ryss.).

- turgida Kotz. (Ord. Sh.).

- Zebra Ktz. (Ord. Sh.).

Eunotia Arcus Ehrb. (Ord. Stav.).

- tridentula Ehrb. var.bidentula W. Sm. Stav.

- gracilis Rab. (Ord. Sh. Stav.).

- impressa Ehrb. (Ord.).

- incisa Greg. (Stav.).

- robusta Ralfs. (Stav.).

Fragilaria brevistriata Grun. (Sh.).

- construens Grun. (Sh.). 
Gomphonema acuminatum Ehrb. (Ord. Sh. RỴss.Stav.)

- capitatum Ehrb. (Ord. Sh).

- constrictum Ehrb. Ord.Sh. Ryss.Star.).

- geminatum Ag. Ryss. Stav.).

- intricatum Ktz. (Ord.).

-. subtile Ehrb. (Sh.).

Mantzschia amphioxys Grun. (Ord.).

Jelosira crenulata Kitz. (Ord.).

- granulata Ehrb. (Rirss.).

Taricula amplithynclus Ehrb. (Sh.).

- Brebissonii Ktz. (Ord.).

- cuspidata Ktz. Sh. .

-- dicergens IV. Sm. (Star.).

- firma Ktz. (Sh.).

- gastrum Donk. (Rirss.).

- gibla Ehrb. (Sh.).

- gigas Ehrb. (Sh.).

- hemiptera Ktz. (Rrss.).

- Mitcliocliï Ehrb. (Rirss.).

- Tridis Ehrb. (Rryss. Star.).

- Legumen Ehrb. (Ord. Sh. Star.).

- limosa Kitz. (Ord. Sh.).

- major Kitz. Ord. Sh. Riss. Stav. .

- mesolepita Ehrb. (Sh.).

- nobilis Ehrb. (Ord.).

- oblonga Kitz. Ord. Rirss.).

- parra Grun. (Ord.).

- producta W. Sm. (Sh.).

- pseudobacillum Grun. (Riss ).

-. radiosa Ktz. (Ord. Sh. Ryss. Stav.).

- spharoptiora Ktz. (Ord.).

- viridis Ktz. (Sh.).

- vulpina Ktz. (Sh. Ryss. Star.). 
Rhopalodia gibla Elırb. Ord. Sh. Star.).

- gilberula Ehrb. (Stav.).

Stauroneis anceps Ehrb. (Sh.).

- Phenicenteron Ehrb. (Ord. Sh. Stav.).

Stephanoliseus Astreea Ktz. (Ryss.).

Suriellu lifions Ktz. (Rirss.).

- rolusta Ehrb. (Riss. Stav.).

- splendida Ehrb. (Sh.).

St,nedra capitata Ehrb. (Ord. Sh. Riss.).

- Clna Ehrb. (Ord. Sh. Rirss.).

Tabillaria fenestrata Ktz. (Ord. Sh. Rysss.).

Le nombre des Diatomées communes aux argiles miocènes du Plateau Central et aux dépôts comparés est de 80; Ordie en contient 33, Sheene 42, RysshyCalmar 35 et Stavanger 22. L'ensemble de cette florule appartient aux dépôts d'eau douce des climats froids.

La présence exclusiye, dans Ryssby-Calmar, de l' $1 \mathrm{~m}$. phora Pediculus, du Cucconeis Placentula, du Cymbella leptoceras, du Diploneis elliptica, du Nacicula Hitchockii et surtout du Stephanorliscus Astrcea, dénote une formation plus ou moins saumâtre et antérieure à celle des autres trois dépôts; mais, ne pouvant vérifier le fait, nous devons nous borner à le signaler à l'attention des diatomistes du Nord, en particulier à M. Ern. Östrup, notre excellent correspondant de Copenhague.

Ces mêmes diatomistes devront rechercher, dans les dépôts de leur région, ou à l'état vivant, les espèces suivantes, à tendances boréales, et trourées dans nos argiles miocènes; telles sont :

Cymbella alpina Grun.

- arctica Lag.

- aspera Ehrb. - Trouvé vivant en Is]aude et dans l'Amérique boréale. 
Diatoma hyemale Heib.

Eunotia parallela Ehrb.

- polyglyplis Grun. var. polydentu'a Ehrb.

Fragilaria elliptica Schum.

- Harrisonï Grun.

- rqualis Lag.

- lancettula Schim.

- Tapponica Grun.

Gomphonema arcticum Gr. - Habite les mers du Nord. - Kamtschaticum Grun.

Melosira distans Kìtz. var. alpigena Gr. - Vorrège, Orégon.

- - var. scalaris Grun. - Orégon.

- Sol Ktz. - N'a été trouvé vivant que dans les mers des régions du Nord.

- spiralis Ehrb. - Orégon.

- tenuis Grun. - Lac Erié (Canada).

Navicula amphibola Clève.

- borealis Ktz.

- lanceolata Ktz.

Synedra liyperborea Grun.

- rumpens Grun.

Tan-Heurckia rhomboides Elub. var. amphipleuroides Gr.

L'ensemble de ces deux listes constitue la florule boréale des argiles miocènes à Diatomées du Plateau Central.

FLoRE DIATOMIQte DE Nos ARUILES MIOCÈNES COMPARÉE A CELLE DU DÉPOT D'Earl Tou'n Lalie (Canada)

Les diatomistes placent le dépôt lacustre d' Earl Toun Lake dans le Quaternaire. Les espèces qui lui sont communes arec nos argiles miocènes ì Diatomées sont : 
Ampliora librjea Ehrb.

Cocconeis Tineata Grun. var. euglypta Girun.

Cymbella cistula Hempr.

- cuspidata Ktz.

- Ehrenbergü Greg.

- lanceolata Ehrb.

Diploneis elliptica Ktz.

Encyonema lumula Grun.

Epithemia turgida Ktz. var. granulata Grun.

- Zebra Ktz.

Eunotia Arcus Ehrb.

Gomphonema acuminatum Ehrb.

- constrictum Elirb.

Ilantzschia amphioxys Grun.

Melosira arenaria Moor.

- granulata Ehrb.

Saricula amphirleynchus Ehrb.

- cuspidata Kiz.

- firma Ktz.

- gibba Ehrb.

- gigas Ehrb.

- gracitis Ehrb.

- Hitchochï Ehrb.

- limosa Ktz.

- mesolepta Ehrb. var. stauroneiformis Grun.

- peregina Heib.

- pseudobacillum Grun.

- Pupula Kitz.

- radiosa $\mathrm{K}$ tz.

- transiersa A. Sch.

- ventricosa Donk.

- viridis Ktz.

Rhopalodia gibba Ehrb. 
Stauroneis acuta W. Sm.

- anceps Ebrh.

- gracilis W. Sm.

- Plicenicenteron Ehrb.

Surirella rolusta Ehrb.

Cette liste comprend 38 espèces, parmi lesquelles 30 sont mentionnées dans les dépôts précédents du Nord de l'Europe. L'analogie qui existe entre les florules de ces formations diatomifères, appartenant à deux continents différents, est une preure que l'aire de dispersion des Diatomées est beaucoup plus générale que celle des Phanérogames.

DIATOMÉES DIS ARGILES MOClENES DU PLATEAC CENTRAL, MTANT DANS LES EAUX DOLCES ET SAUMATRES ACTLELLES DE QUELQUES RÉgIONS É?CATORIALES.

La liste suivante a été établie d'après l'étude de récoltes faites à l'Equateur = E., à la Nouvelle-ZÉLANDE $=Z$ et au SIAM $=\mathrm{S}$.

Les Diatomées observées sont:

Achnanthes lanceolata Grun. (E. S.).

Cocconeis Placentula Ehrb. (Z.).

Cyclotella Meneghiniana Ktz. (S. Z.).

- stelligera Grun. (Z.).

Cymbella affinis Ktz. (Z.).

- gastroides Kitz. (Z.).

-- tumidula Grun. (E.).

Epithemia Sorex Ktz. (Z.). 
Eunotia lunaris Grun. (S.).

- diodon Ehrb. (Z.).

-- paralleta Ehrb. S.).

- pectinalis Rab. (E. S. Z.).

Gomplionema affinis Ktz. (E.).

$$
\text { - miciopus Ktz. (S.). }
$$

Ilantzschia amphlioxys Grun. (S.).

Melosina granulata Ehrb. (Z. .

Sireicula ampleigompleus Ehrh. (Z.).

- anjlice Rialís. (E.).

- Bramii Grun. S.).

- cincter Ktz. (S.).

- diceplecta W. Sm. (E.).

- dicergens IT. Sm. (S.).

-- Hitclenclï Elırb. (S.).

- Leguimen Ehrb. (Z.).

- major Ktz. (E. Z.).

- parea Grun. (S.).

- Pupula Ktz. E. S.).

- rarliosa Ktz. (E. S.).

-. rhyncocephala Krtz. (Z.).

Stephanodiscus Astreea Ktz. var. mimutula Gr. Z.). Surivella tenera Greg. E.

Synedra Chu Ehrb. var. vitrea Ktz. (Z.)

Pour compléter la florule tropicale de nos argiles miocìnes à Diatomées, il nous reste à mentionner quelques autres espèces trouvées dans diverses régions chaudes du globe et qui ne figurent pas daus la liste précédente, telles que:

Actinella pliocenica F. Hérib., analogite de l'Astinetla Lrasitiensis Grun. -- Trouvé fossile it Sendaï Japon) et vivant au Brésil. 
146 DISTRIBCTION GÉOGR. DES DIAT. TERT. DU PLAT. CENTRAL

Cymbella australica A. Sch. var. fossilis nor. - Le type n'est connu vivant qu'en Australie.

Cymbella turgidula Grun. - Trouvé vivant aux Antilles et aux Indes.

Gomplonema lrasiliense Grun. var. fissilis nov. La forme trpe est particulière au Brésil.

Nacicula cellesensis F. Hérib., analogue du Naricula instabilis A. Sch. - Constaté vivant à la Guyane.

Naricula Porrecta Ehrb. - Trouvé vivant dans l'Amérique centrale.

Melosira granulata Ehrb. var. australiensis Grun. N'a été observé vivant qu'à la Nouvelle-Hollande.

Opepthora Martyi F. Hérib. et cantalense F. Hérib. - Les formes analogues n'habitent que le Brésil.

Stauroneis parrula Grun., l'analogue de notre Stauroneis antediluriana, n'est connu que dans le guano du Pérou.

D'après ces deux listes, la florule tropicale des dépôts miocènes à Diatomées du Plateau Central comprend 42 espèces. 


\section{COMPARAISON}

DE LA FLORE DES ARGILES MIOCENES A DIATOMÉE DE

PLATEAU CENTRAL ATEC LA FLORE ACTUELLE DE JATA

Les projections des rolcans trachytiques de Java arant une très grande analogie avec celles des rolcans du Cantal, des Wonts Dores et du Mezenc, ainsi que l'admettent les géologues, nous arons pensé qu'il serait fort intéressant et très instructif de comparer les flores diatomiques des deux régions.

Grâce à l'amabilité de notre ami, M. le Commandant II. Peragallo, nous pourons donner une bonne liste établie d'après l'étıde de huit préparations, provenant de récoltes très authentiques et faites à des stations direrses, tant sous le rapport de l'altitude que de la nature des eaux.

Les récoltes proviennent: $1^{\circ}$ de la vase d'un cratèrelac trachytique; $2^{\circ}$ de la vase de sources salines fortement minéralisées; $3^{\circ}$ d'un ruisseau dans lequel se déversent des sources minérales très chaudes. Il résulte de ces indications que la plupart des Diatomées ont été récoltées à l'état vivant, et qu'elles se sont développées dans des conditions identiques à celles qui ont donné lieu à la formation des argiles miocènes diatomifères du Plateau Central.

Dans la liste ci-dessous, les noms des Diatomées constatées dans nos argiles miocènes sont imprimés en 
égyptien; les espèces d'Auvergne qui n'ont pas été observées dans les dépôts sont en itulique; enfin, les Diatomées de Java que nous n'arons pas encore tronvées dans le Plateau Central sont imprimées en romain.

La flore diatomique actuelle de Jara, comprend les espèces suirantes:

Achnanthes crenulata Grun.

- inflata Cirun.

- lanceolata Grun.

- - var. capitata ror.

- - var. dubia Grun.

- microcephala Ktz.

- subsessilis Kitz.

Amphora libyca Ehrh.

Ceratanlus levis Elert.

Cocconeis Placentula Ehrb.

Cymbella delecta A. Sch.

- americana $A$. Sch.

- stomatopliora Grun.

Denticula Tan-Heurckii J. Brr.

Diploneis elliptica Kt

Smithii Bréh.

Encyonema gracile Rab.

- turgidum Grun.

Epithemia Sorex Ktz.

Zebra Ktz.

Eunotia incisa Crreg.

- major Rab.

- parallela Ehr\%.

- pectinalis Rab.

- preverpta Ehro. 
Fragilaria brevistriata Grun.

elliptica Schum.

Harrisonii Grm.

mutabilis Grun.

Gomphonema brasiliense Grun.

$\begin{array}{ll}\text { - } & \text { exiguum Ktz. } \\ \text { - } & \text { Lagenula Ktz. } \\ \text { - } & \text { micropus Ktz. } \\ \text { - } & \text { pagitulum Ktz. } \\ \text { - } & \text { subtile Grum. }\end{array}$

Grammatophora marina Kty.

Stictodiscus pulchellus Grun.

Hydrosera javanica A. Sch.

Masogloia lanceolata Ther.

Melosira distans Ǩtz.

- granulata Ralfs.

- undulata Kítz.

Navicula acrosphæria Bréb.

- affinis Ehrb.

- ambigua Ehrb.

- bacilliformis Grun.

- divergens W. Sm.

- firma Ktz.

- gallica W. Sm.

- gibba Ktz.

- Legumen Ehrb.

- major Kiz.

- mesolepta Ehrb.

- - var. stauroneiformis Grun.

- radiosa Ktz.

- rostellata Grun.

- transversa A. Sch.

- viridula Ktz. 
Nitzschia Frauenfeldii Grun.

Heufleriana Grun.

Pleurosigma Karianum Clère.

Rhopalodia gibba Ktz.
$\rightarrow$ gibberula Ktz.

Stauroneis javanica Grun.

- - var. arvernense nov. l'analogue du type de Java.

- Phœnicenteron Ehrb.

- phṛllodes Elerb.

Surirella apiculata $W^{*}$. S $m$.

- linearis $\mathrm{W}$. Sm.

- tenera Greg.

- - var. splendidula Greg.

Synedra delicatissima W. Sm.

- notata Grun.

- rumpens G. rar.fragilarioides I.ll.

- Smithiana Grun.

- Ulna Ehrb.

- - var. subæqualis Grun.

- - vár. lanceolata Kítz.

Tetracyclus sp.?

Van-Heurctia vulgare Grun.

II. le Commandant MI. Peragallo, considère cette série de Diatomées comme représentant assez exactement la florule diatomique actuelle de Jara.

Notre liste compte 81 espèces et variétés, dont l'ensemble est un mélange de Diatomées marines, saumîtres et d'eau douce, tout à fait analogue à celui de nos argiles miocènes diatomifères.

Parmi ces 81 Diatomées, 62 appartiennent à la flore du Plateau Central, comprenant 49 espèces fossiles et 13 à la flore actuelle. 
Ainsi, il existe une très grande analogie entre la flore diatomique actuelle de Java et celle de nos argiles miocènes à Diatomées.

Nous sommes donc autorisé à conclure que la formation des argiles miocènes à Diatomées du Plateau C'entral, a dû s'effectuer dans des conditions analogues à celles que l'on constate actuellement à Java.

Si nos conclusions diatomologiques n'ont pas toujours été d'accord arec celles des géologues, ici du moins la concordance est parfaite.

Ehrenberg (Milrogéologie) mentionne 3 Diatomées dans une formation argileuse, connue des géologues sous le nom de Terre glaise comestible de Tanah-AmboSamarang (Jara), dont nous ignorons l'origine et la nature des éléments qui la composent.

Il est reconnu aujourd'hui que les 3 espèces d'Ehrenberg sont :

Mastogloia lanceolataThw.
Melosira distans Ktz. Heurchia vulgare Gr.

Dans une préparation de cette même terre comestible, II. le Commaudant II. P'eragallo a constaté :

Achnanthes subsessitis Ktz. I Varicula major Ktz. Ilelosira granulata Ralfs. Tetracyclus sp.?

La florule de la terre comestible de Java comprend donc 7 Diatomées, donc 5 appartiennent à la flore d'Auvergne. 
Enfin, Ehrenberg ( in Nonatsherichten der Kon. Acad. zu Berlin, 1855, p. 561), signale encore des Diatomées dans les projections boueuses du volcan de Poorwadadi (Java): ce sont:

Amphora sp.?

Campylodiscus Echeneis? Coscinodiscus subtilis sp.?

Frustulia sp.?

Gallionella crenata.

Gallionella sulcata. Hemiaulus sp.?

Naricula sp.?

Synedia sp.?

Le grand nombre de points ? indique combien l'examen du célèbre diatomiste a dû ĉtre incomplet.

La présence de Diatomées dans ces projections boueuses semble à première rue très bizarre; nous ne pouvons expliquer ce fait que par l'infiltration des eaux marines dans les couches traversées par le volcan boutux, à moins d'arlmettre le passage des émissions boueuses à travers des terrains sédimentaires à Diatomées, lesquelles seraient ainsi ramenées à la surface du sol.

La plupart des espèces de Java que nous n'arons pas olsservées chez nous sont marines; leur présence dans les eaux javanaises s'explique par la situation insulaire de ces rolcans trachytiques, et le mélange des eaux marines avec les projections volcaniques. 


\title{
APPLICATIONS INDESTRIELLES
}

\author{
DLS DLATOMEES FOSSILLS
}

\section{A quoi servent les Diatomées?}

Telle est la question qui nous a été souvent adressée au cours de nos études diatomiques; nous laisserons à notre très distingué compatriote, M. Pagès-Allary, le soin de donner la réponse.

\section{La voici :}

“Outre l'emploi lien connu des Diatomées fossiles, larcées et calcinces, pour la fabrication de la dynamite, elles sont utilisées, ccmme alsorlant, par les fabricants de couleurs, cires à cacheter, cirages, pâtes à papier, caoutcheuc, gommes à effacer, corps gras et acides en général; on les substitue arantageusement à l'argile à foulon dans les fabriques de draps; elles servent à former les meilleurs filtres pour l'ean, les huiles, les sirops et les liquides acides; les fabricants d'engrais chimiques les emploient pour l'alusorption des éléments fertilisants solubles; elles peuvent et derraient partout remplacer la paille et la tourbe comme litière; cette application mérite d'être mieux comprise des proprićtaires cultivateurs de la région montagneuse, où la paille est rare et toujours d'un prix élevé. 
Iais c'est surtout dans l'industrie des produits réfractaires, isolants et aphones, que les Diatomées fossiles trouvent les applications les plus importantes, telles que : briques réfractaires et légères 250 à 300 kilogrammes le mètre cube) pour voûtes de four, chaudières marines, etc.; briques aphones pour cloisons, travaux de hourdis, plafonds, etc.; isolants de toute nature pour l'électricité et la chaleur, d'où leur utilité pour les glacières, les navires à conserver la viande, les fruits: les calorifuges pour chaudières, tuyaux de rapeur, etc. Nous les trourons encore dans les produits à polir: tripoli (très supérieur au tripoli composé de silice amorphe), pâtes, savons minéraux, polissage du bois, de l'écaille et des métaux; leur emploi dans la fabrication des stucs, des céruses, des tuyaux de drainage, des carreaux émaillés, des tuiles légères, etc. )

Enfin, nous ajouterons que ce sont les Diatomées, " ces pierres de touche des objectifs, qui ont fait faire à l'art difficile de la construction du microscope plus de progrès que tous les êtres réunis de la création. )

Si nos modestes travaux ont quelque peu contribué à la connaissance des dépôts à Diatomées du Plateau Central, nous arons aujourd'hui la satisfaction de constater que nos labeurs n'ont pas été tout à fait inutiles à nos compatriotes.

Les rastes dépôts miocènes du Cantal, inconnus arant 1890 , sont actuellement l'objet d'une exploitation très importante, solidement établie, et dirigée avec beaucoup d'intelligence par deux honorables compatriotes, MII. Pagès-Allary et Rhodes; nous sommes persuadé que l'arenir répondra pleinement aux espérances du présent. 
Les industriels qui ne veulent employer que des dépôts à Diatomées de qualité alssolument supérieure, doirent s'adresser de préférence à MIII. Pagès-Allary et Rhodes, propriétaires-industriels à Murat (Cantal).

DEO scientiarum Domino laus ct gloria! 

PLANCHE IX 


\section{PLANCHE IX}

FIG.

1. Amphora Borneti nor. sp ; rue valvaire aus 3, 4 , p.68 et 70.

2 . - distincta nor. sp.. p. 15.

3. Crmbella C'reguti nor: sp., p. 17.

4. - Laubri nor.sp., p. 18.

b. - meniscus nov. sp., p. 18.

6. - capitata nov. sp., p. 50.

7. - Foucaudi nov. sp., p. 16.

8. - Harioti nov. sp., p. 15.

9. Encyonema Grandi nov. sp., p. 20.

10. Naricula major Ktz. rar. convergentissima nov., p. 10.

11. Crmbella Rhodesi nov. sp., p. 72 .

12. - Brerieri nox.sp., p. 16.

13. - radiosa noc.sp., p. 15.

14. - Pagesi ner.sp., p. 71.

15. Naricula jolirsacensis nov. sp., p. 8 .

16. - Corbieri nor. sp., p. 7 .

17. - Costei nor. sp., p. 9.

18. - Chaberti nov.sp., p. 60.

19. - Costei rar. bacillaris nov., p. 10.

20. - triangulifera nov. sp., p. 9.

21. - Lereillei nox, sp., p. 12.

22. - Esox Ehrb. var. recta nor., p. 11.

23. - Olivieri nov. sp., p. 11.

24. - Berriati nov. sp., p. 13.

25. - - rar. minor nox., p. 14.

26. - Huei nor. sp., p. 62.

27. - - decurrens Ehrb. var. curtecostata nor', p. 12.

28. - dubitata nov. sp., p. 6.

29. - ventricosa Donk. var. cuneata nov., p. 59.

30. - pseudo-bacillum Grun. var. elapsa nov., p. 5.

31. - Gendrei nou'sp., p. ‘.

32. Stauroneis quadrata nox. sp., p. 14 . 
Dialomées d'Auvergne

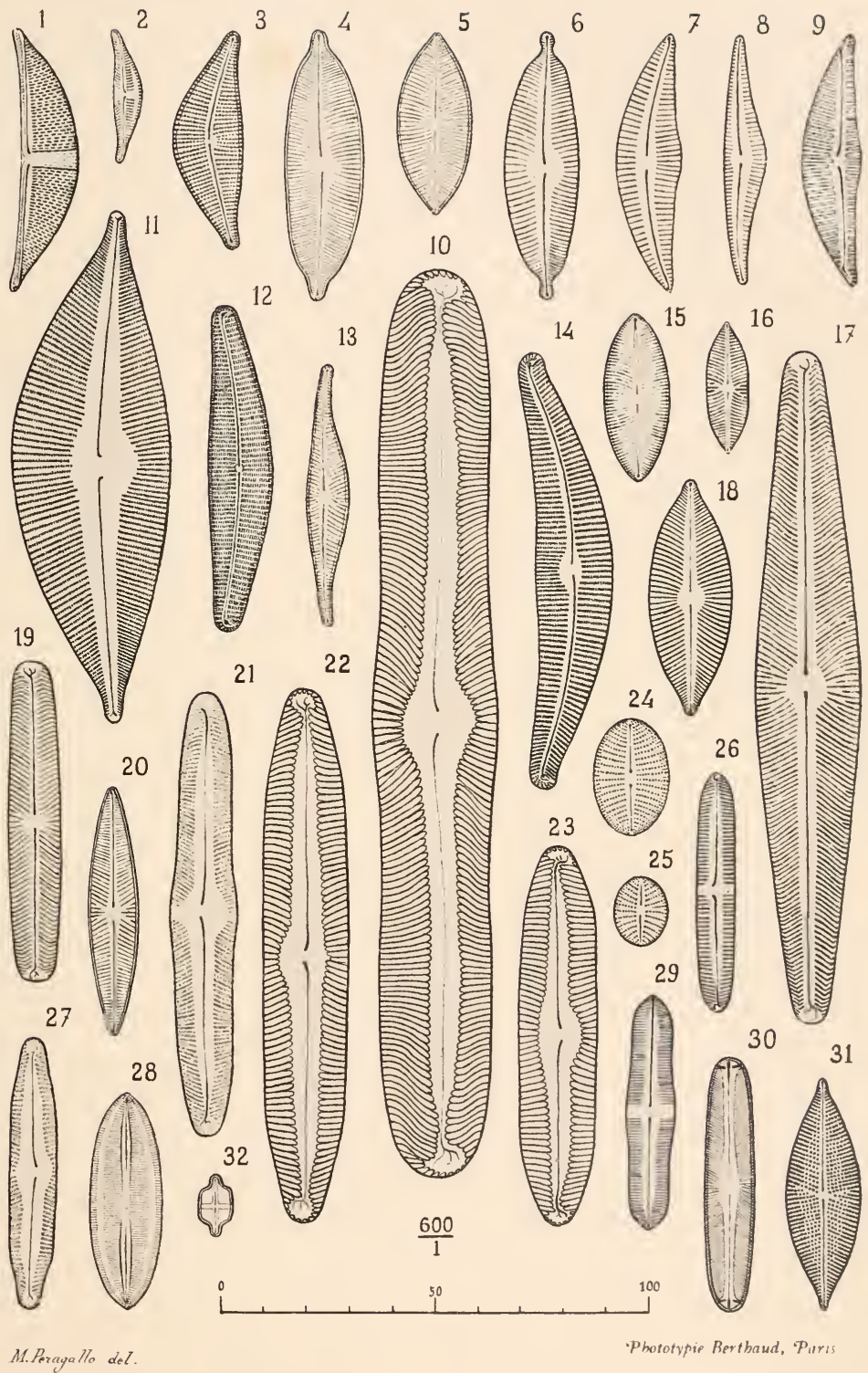



PLANCHE I 


\section{PLANCHE $\mathrm{X}$}

Fig.

1. Gomphonema rigidum nor. sp., p. 78.

2-8. - - accessum nor. sp., p. 23.

4. - - parrmm. vor.sp., p. 22.

5. $\quad$ - latestriata nox.sp., p. 20.

6. - exscissum nor.sp., 1.2.2.

7. $\quad-\quad$ Gilloti nor. sp., 23.

8. - - Licmophoraoides nor. sp., p. 24.

9. Fragilaria Zeilleri nor. sp., p. 26 .

10. - - rar. nitzschioides nor., 1. 27.

$10^{\text {this }}$ - $\quad$ - forma anomala nov.. p. 27.

11. - brevistriata frun. var. elliptica nov., p. 74 .

12. - Harrisonii firun. var. major nov.. p. 25.

13. - Gustarci nor. $s p .$, p. 25.

14. Opephora cantalense noi. sp.; $a, b, c$, vie connective; $d$, vue ralvaire, p. 30.

15. - - rar. capitata nor.: rue ralraire, p. 30.

16. - Martri rar. capitatanor'; rue valraire, p. 30.

17-18. Achnanthes Flahaultinou'.sp;; 17 , valre inférienre; 18 , valre supérienre, p. 57.

19. Epithemia turgida Ktz. rar. porecllus nor., p. 50 .

20. Eunotia polçdentula Ehrb. rar. fossilis nov., p. 28.

21. Tetractclus Peragalli nor. sp., p. 81.

22. - - $\quad$ - rar. major nor.. p. 81 .

23. - - rar. eximia nor., p. 31.

21. Surirella Pagesi not'. sp, 1. 29.

25. Crmnowia moissacensis nor. sp., p. 75.

26. Cymatopleura Martyi nov. spo, p. 75.

27. Melosira mudulata Kítz. var.debilisnor',; rue comnective, p. 82.

28. - - rar. debilis et producta rémies, p. 28 .

29. - Camusi noe. sp.; b, rue connectire, p. 33.

30. - - - rar. conica nov.; rue connectire, p. 34.

31. - imperfecta noz.sp., p. 32 .

32. - minuta nov.sp.. p. 32.

83. - lineolata rar, robnsta nor., p. $7 \%$.

34. - canalifera rar. anastomosans nou', p. 52.

35. Cyclutella perforata nor. sp.; $b$, rne connective, p. 34 .

36. - Kutzingiana Chaur. rar. gemmatulata noz'., p. 7 . 
Dialomées d'Auvergne

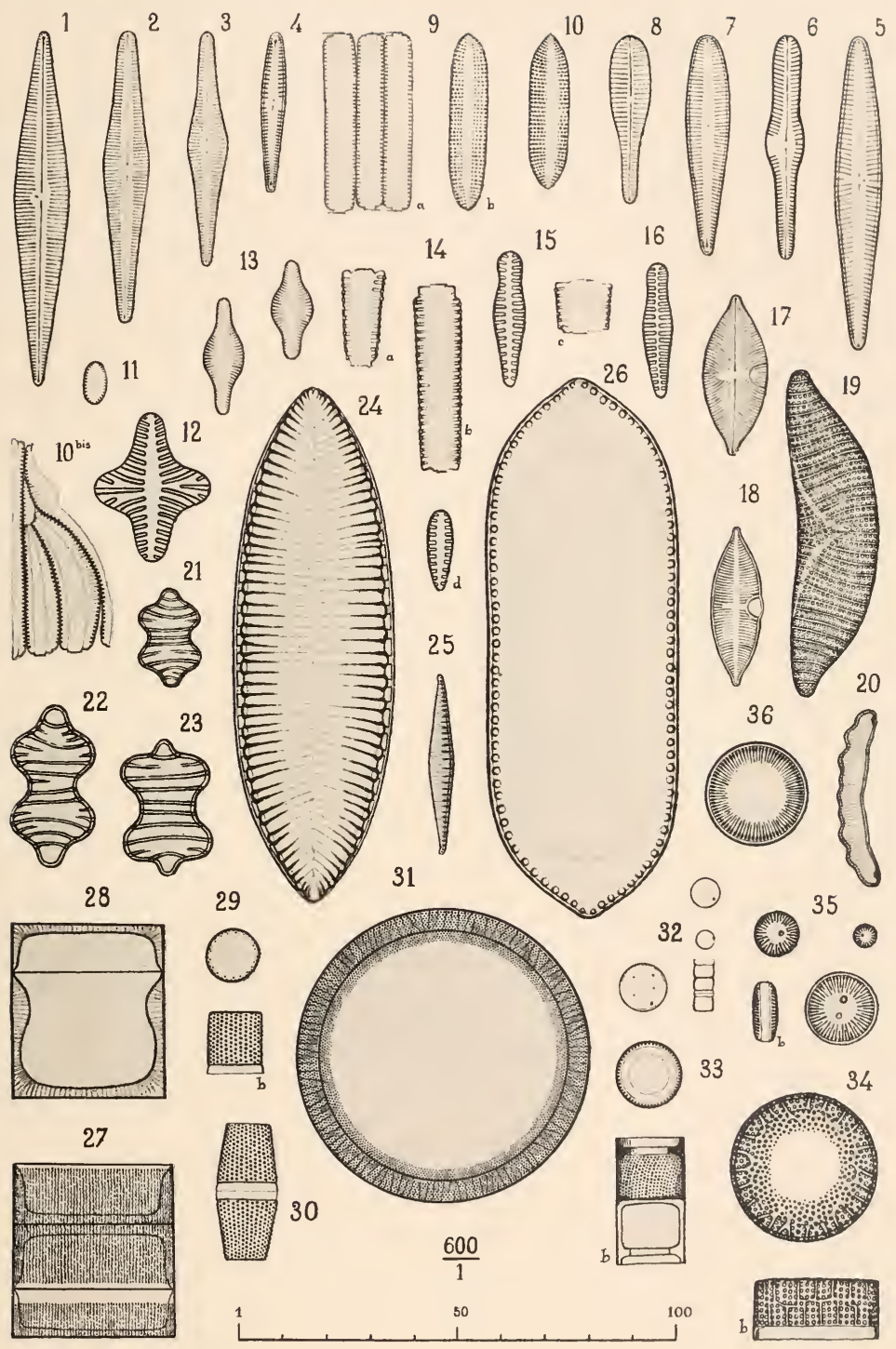

M.Peragallo del. 

PLANCHE XI 


\section{PLANCHE XI}

Fig.

1-3. Amphora Borneti nor. sp.; 1, vue ventrale; 2 , rue valvaire aux $34 ; 3$, vue dorsale, p. 68 et 70 .

4. Cymbella Hauckii V.-H. var. fossilis nov', p. 72 .

5. Encyonema intermedium nov. sp., p. 19.

6. Stauroneis antediluviana nov. sp., p. 66.

7. Diatoma hyemale Heib. var. acuta nov., p. 81 .

8. Stauroneis javanica Grun. var. arvernense nov., p. 66.

9. Navicula ventricosa Donk. var. decrescens nov., p. 6.

10. - bicapitata Lag. var. sulcata nov., p. 63.

11. - hemiptera Ktz. var. gibba nor', p. 65.

12. - divergens W. Sm. rar. undulato-cuneata nor., p. 62.

13. - acrosphæria Bréb. var. bacillaris nov., p. 64 .

14. - - var. elongata nov., p. 64.

15. - gibbula Clère var. cantalicanov., p. 59.

16. - digito-radiata Greg. var. obesa nov., p. 9.

17. - Braunii Grun. var. moissacensis nor., p. 63.

18. - acephala nov. sp., p. 61.

19. - decrescens nox. sp., p. 63.

20. - hemiptera Ktz. var. angusta noz', p. 64.

21. - peregrina Heib. var. fossilis nov., p. 79.

22. Gomphonema breristriata nov, sp., p. 80 .

23. Tetracyclus stellare nov. sp., p. 31.

24-25. Cocconeis Bonnieri nov. sp., p. 59.

26-27. Achnanthes joursacense nov. sp., p. 5.

28. Sțnedra Ramesi nov. sp., p. 80.

29. Fragilaria nitida nov. sp., var, delicatula nov., p. 27.

30. - nitidanov. sp., p. 27.

31. Surirella gracilis Grun, var. constricta nov., p. 29. 
Dialomées d Auvergne

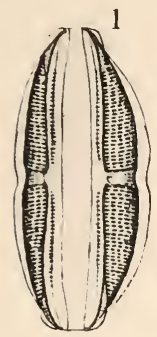

$\overbrace{}^{16}$
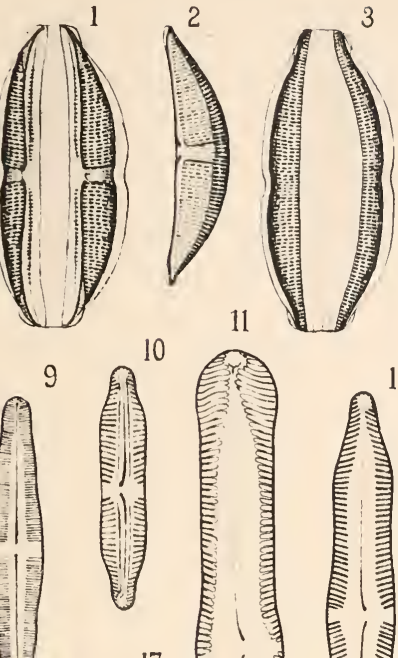

11

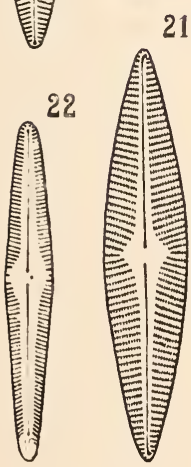

M.Peragallo dé:

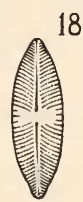

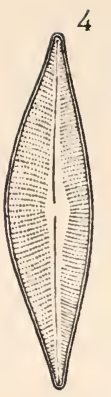
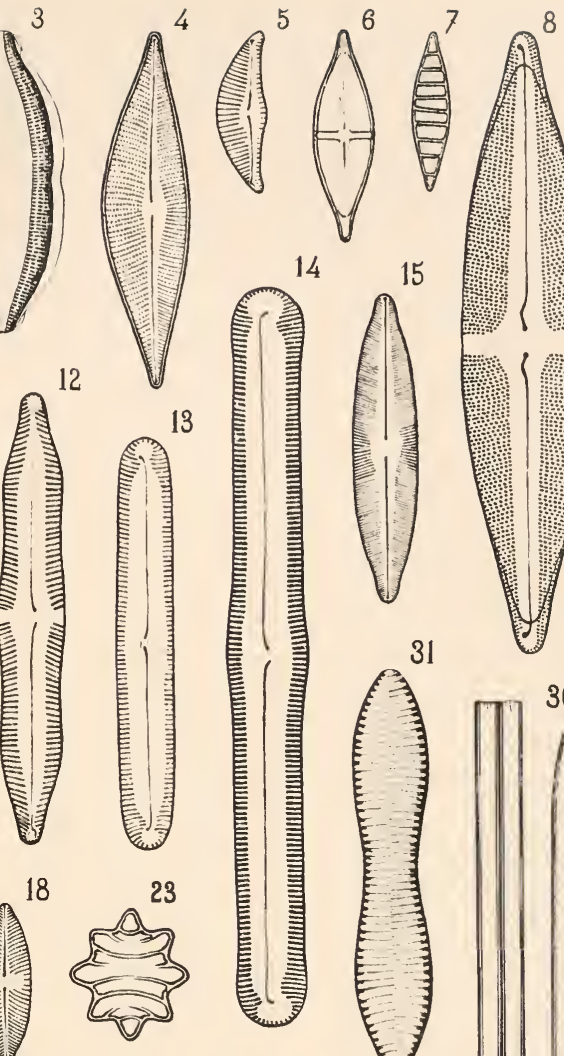

覧

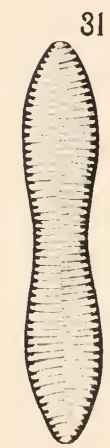

31
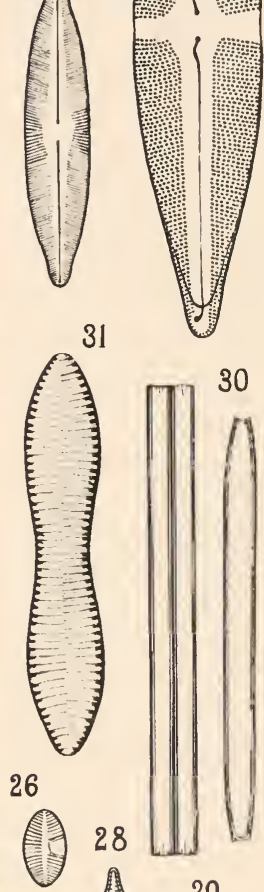

28

量 $\quad 29$

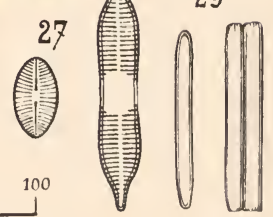

'Phototypie Berthaud, Paris. 
PLANCHE XII 


\section{PLANCHE XII}

FIG.

1. Navicula radians nov. s.., p. 90 .

2. - halophila Grun. var. major nov., p. 89.

3. - mesolepta Ehrb. var. Alberti nov., p. 90.

4. - Renauldi F. Hérib. var. major nov., p. 13.

5. - - lambertensis nov. sp., p. 104.

6. - Placentula Ehrb. var. major nov, p. 40.

7. - Pupula Ktz. var. major nov., p. 39.

8. Synedra joursacensis nov. sp., p. 24.

9. Tetracyclus Boryanus (Pant.) var. minor nov., p. 51.

10. Gomphonema eriense Grun. var. acuminata nov., p. 21.

11. - brasiliense Grun. var. fossilis nov', p. 105.

12. Cymbella Laubyi F. Hérib. var. lanceolata nov., p. 40.

13. - Bruyanti nov.sp., p. 105.

14. - australica A. Sch. var. fossilis nor., p. 104.

15. Actinella pliocenica F. Hérib. var. tenuistriata nov', p. 44.

16. Fragilaria bigibba nov'sp., p. 92.

17. Ceratoneis antiqua nov, sp., p. 106.

18. Fragilaria brevistriata Grun. var. capitata nov.. p. 92.

19. - Zeilleri F. Hérib. var, densestriata nov., 1. 92.

20. Achnanthes ligeriana nov. $s p$., p. 89.

21. Opephora Martyi F. Hérib. var. robusta nov', p. 41.

22. - - forma anomala nov., p. 41.

23. Melosira bellicosa nov. sp., p. 93.

24. - sphærica nov. $s p . ; a$, vue connective; $b$, vue valvaire.

25. - granulata et canalifera; deux frustules réunis en vue connec.ive nov., p. 53.

26. Cyclotella comta Ktz. var. trinotata nor., p. 95.

27. - - var. quadrinotata nov', p. 100.

28. - - var. quinquenotata nov., p. 108.

29. - Guignardi nov. sp., p. 107.

30. - - antiqua W. Sm. var. Vernierei nov., p. 107.

31. - comta Ktz. var. decrescens nov., p. 95.

32. Coscinodiscus Boulei H. Peragallo, p. 101. 
Dialomées d'Auvergne

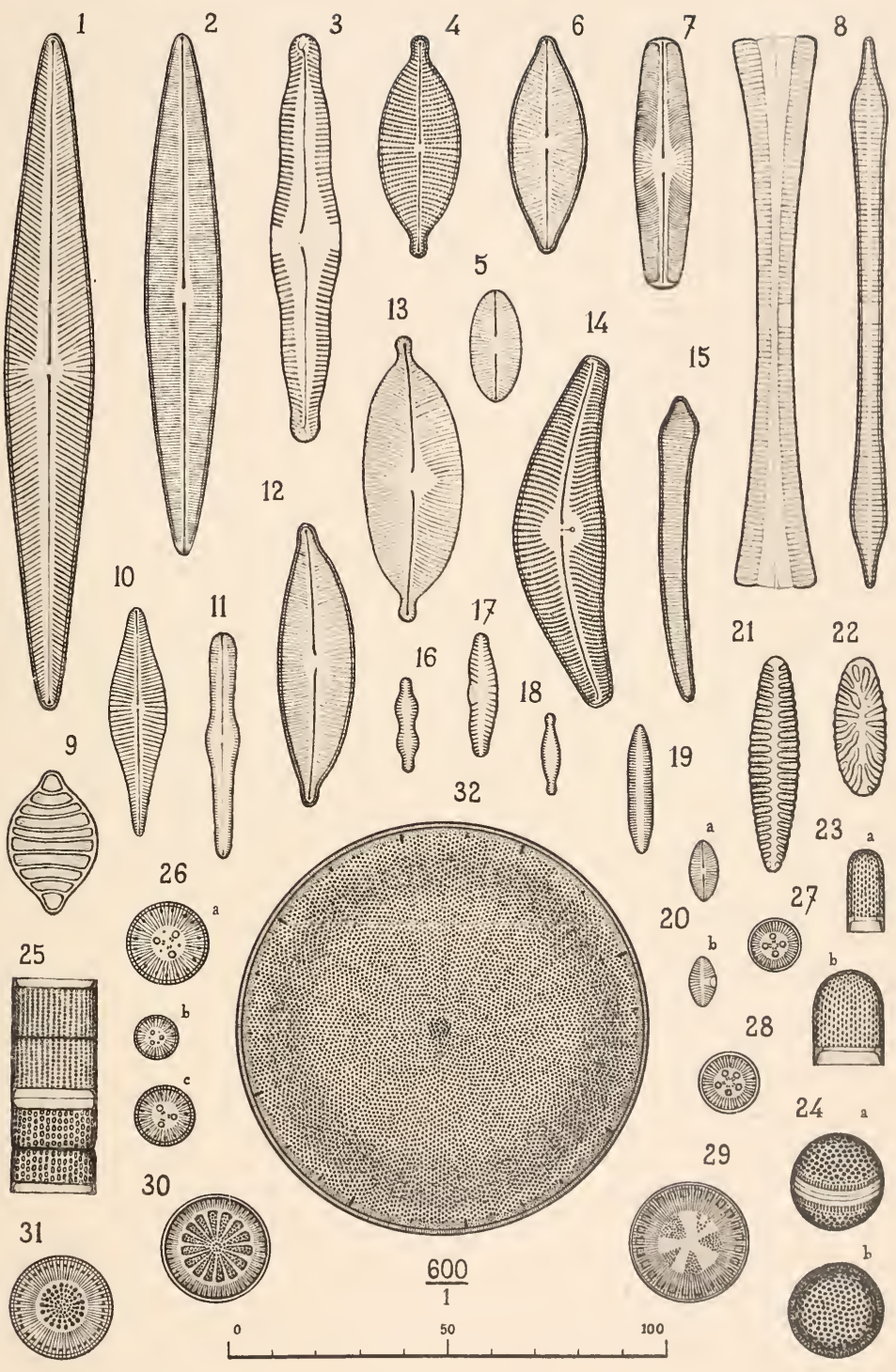

M. Peragallo del. 



\section{TABLE DES MATIERES}

Préface.......................... $v$

Dépots de Cantal ....................... 1

Dépôt de Joursac....................... 1

Florule phanérogamique de Joursac............. 36

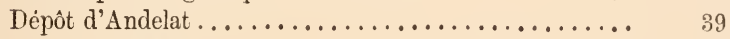

- de Chambeuil....................... 43

- de Fraisse-Bas ..... ... ............. 46

- de Faufouilhoux.................... 49

- de Sainte-Anastasie ................... 54

— de Moissac .........................

- de Neussargues. .................... . . 79

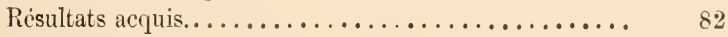

DÉPOTS DE la HAUte-LoIRE................. 87

Dépôt de Ceyssac................... 88

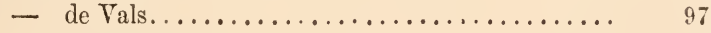

— du Monastier...................... 99

- de la Roche-Lambert. ................. 103

DÉPUT DE L'ARDĖCHE .................... 111

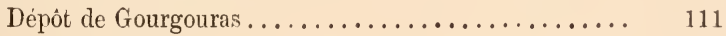

- de Charay......................... 113

- de Ranc........................... 118

- de Pourcheres...................... 120

Odservations sur les Dépots tertiatres a Diatonées du Plateau Cextral..................... 125

Florule des espéces marines et saumâtres de nos argiles tertiaires à Diatomées................... 


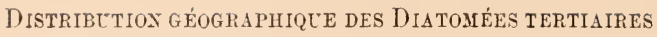
de Plateat Central..................... 138

Diatomées de nos argiles miocènes comparées à celles de quelqus dépôts du nord de l'Europe.............

Flore diatomique de nos argiles miocènes comparée à celle du dépôt d'Earl Town Lake (Canada)...........

Diatomées des argiles miocènes du Plateau Central, rivant dans les eaux donces et saumatres actuelles de quelques régions équatoriales................

Comparaison de la Flore des argiles miociènes a Diatomées de Platead Cextral afec la Flore

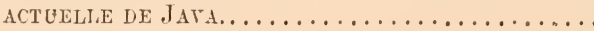

Applications indéstrielles des Diatomées fossiles. .

PLANCHES IX, X, XI, XII 


\section{LES DIATOMEES FOSSILES}

D'AUVERGNE 



\title{
LES
}

\section{DIATOMÉES FOSSILES}

\author{
D'A UVERGNE.
}

/Troisième Mémoire'

PAR

\section{Le Frère HÉRIBAUD JOSEPH}

LAURÉAT DE LINSTITUT DE FRAN('E

(Académie des sciences;

CORRESPONDANT DU MUSÉUM

MFMBRE HOXORAIRE DE L'ACADEMIE DE CLERMONT-FERRAND

ET DE IA EOCIÉTÉ BOTANIQVE DE FRAXCE

\author{
Déo scientiarum Domino \\ laus el gloria.
}

AVEC 2 PLANCHES

DESSINÉES PAR LE COMMANDANT MAURICE PERAGALLO

Prix : 5 Francs

PA Ri Is

LIBRAIRIE DES SCIENCES NATURELLES

RAUL KLINCKNIECK

3, rue Corneille, 3

1908 



\section{PRÉFACE}

Nos premières recherches sur les Diatomées datent de 1888; iuterrompues de 1893 à 1899, par la publication des Muscinées d'Auvergne, l'étude minutieuse de ces petites Algues a été reprise en 1900, et poursuivie jusqu’à présent avec toute l'activité possible.

L'examen d'un grand nombre de récoltes de Diatomées vivantes ne nous ayant donné que des formes déjá mentionnées dans le Mémoire de 1893, nous avons négligé leur étude dès le prin. temps de 1900, pour nous oceuper exclusivement des Diatomées fossiles, bien plus intéressantes pour nous que les espèces actuelles, surtout au point de vue industriel.

Dans le but de prendre date de nos découvertes, et établir ainsi notre droit de priorité concernant les espèces nouvelles, nous avons dû publier successivement les Mémoires de 1902, 1903 et 1908; ces trois publications constituent les 
parties d'un travail continu, relatif à une même question, et ne doivent pas être séparées; elles contiennent, avec la totalité des espèces inédites, décrites avec le plus grand soin, et figurées dans 8 bonnes planches, l'exposé de plusieurs faits acquis à la science, tels que :

Influence de la lumière et de l'altitude sur la striation des valves des Diatomées.

Recherches sur la formation des dépôts à Diatomées.

Observations sur les dépôts tertiaires du Plateau Central (Némoire de 1903, p. 12:3). Sous ce titre, les paléobotanistes trouveront l'exposé de notre théorie sur la fossilisation des feuilles et autres objets plus ou moins délicats.

Explication de l'absence absolue de feuilles fossiles dans les dépôts quatemaires de la chaîne des Monts Dômes, tandis qu'elles sont toujours plus ou moins abondantes dans les dépôts tertiaires du Cantal, des Monts Dores, de la HauteLoire et de l'Ardèche.

Remaniement et restratification des dépôts tertiaires; différence d’âge des deux florules diatomique et phanérogamique d'un même dépôt.

Explication rationnelle de la présence, dans ces dépôts, des Diatomées marines ou saumâtres. 
Comparaison de la flore diatomique des dépôts tertiaires du Massif Central, avec celle des dépôts similaires de l'Europe boréale (Ecosse, Suède et Norvège), et de quelques dépôts de la zone équatoriale.

Analogie de la flore diatomique des dépôts tertiaires du Plateau Central avec la flore actuelle de quelques lacs de Java, alimentés par de puissantes sources thermo-minérales, comme devaient l'être nos cratères-lacs trachytiques, dans lesquels se sont formés les vastes dépôts diatomifères, dont il ne nous reste aujourd'hui que des lambeaux restratifiés par les eaux à l'époque du Miocène supérieur.

Les faits que nous venons d'énumérer, et qui se trouvent exposés dans les Mémoires de 1902 et 1903, sont pleinement confirmés par les observations faites au cours des cinq dernières années.

Avec la publication de ce troisième Mémoire, les Diatomées fossiles de nos dépôts quaternaires et tertiaires sont maintenant assez bien connues, et nous n'espérous pas que les recherches ultérieures ajoutent notablement aux faits acquis pendant les quinze années consacrées à l'étude de ce groupe d'Algues inférieures; mais il resterait encore à rechercher les espèces fossiles des 
travertins déposés par les sources pétrogéniques, très nombreuses en Auvergne; cette étude présenterait d'ailleurs un très grand intérêt, parce qu'elle nous permettrait de constater les variations successives de la florule diatomique de chaque source minérale, depuis les temps les plus reculés jusqu’à la florule actuelle; de plus, la nature des espèces observées pourrait fournir des indications précieuses sur les variations que les eaux de ces sources ont pu subir depuis leur exploitation par les Romains (Royat, Mont-Dore, Saint-Nectaire, etc.) jusqu’à notre époque.

Nous n'avons jamais observé de Diatomées marines, ni même de formes franchement saumâtres dans les sources minérales actuelles; il serait donc très intéressant de trouver, dans les travertins anciens de ces sources, des espèces appartenant à des genres marins, comme les Amphiprora, les Raphoneis, les Striatella, les Stictodiscus, les Terpsinuë, les Goniothecium, etc., ou nettement saumâtres, comme les Coscinodiscus, les Stephanodiscus, etc., qui sont assez fréquents dans les dépôts tertiaires du Plateau Central; la constatation de ces Diatomées dans le dépôt d'une source pétrogénique prouverait qu'elle avait antrefois un degré de minéralisation 
très supérieur à celui qu'elle possède aujourd'hui, puisqu'elle est trop pauvre actuellement en éléments salins pour permettre à ces microorganismes de vivre et de se multiplier.

En 1890 nous avons trouvé, à l'état virant, dans la source minérale de St-Floret (Puy-deDôme), le rarissime Nitzschia Krittlii Grun. (Diat. d'Auv., Pl. V, fig. 2 et 3), très belle espèce qui n'était encore connue qu'à l'état fossile en Hongrie; or, dans un échantilion du travertin déposé par cette source, pris à 2 mètres de profondeur, nous avons retrouvé le même Nitzschia fossilisé, preuve bien évidente que depuis plusieurs siècles cette Diatomée existe dans l'eau minérale de StFloret.

Ce fait nous montre l'intérêt que présenterait la publication des résultats acquis dans ce genre de recherches; ne le pouvant nous-même, à cause du manque absolu da ressources, depuis la sécularisation du Pensionnat de Clermont-Fd (1904). nous sommes à la disposition du diatomiste qui voudrait entreprendre ee beau travail.

Les espèces nouvelles pour la flore générale, déerites et figurées dans les trois Mémoires, sont an nombre de 290, résultat de 8 innées de recherches laborieuses et quelque peu minutieuses. 
Les deux belles planches de ce Mémoire sont dues au talent bien connu et très apprécié de notre éminent ami, le Commandant Maurice Peragallo; nous le prions d'agiréer nos meilleurs remerciements, en retour du service qu'il nous a rendu avec la plus parfaite amabilité.

Nous devons aussi beaucoup de reconnaissance à M. Gallaud, le savant professeur de sciences naturelles du Lycée de St-Etienne, pour avoir bien voulu nous faire l'honneur de nous offrir la primeur de la partie diatomique des dépôts découverts par lui en 1906 et 1907 dans la HauteLoire, et pour nous avoir procuré, arec le plus aimable désintéressement, les matériaux utilisés pour l'étude de ces beanx dépôts remaniés et inédits.

Il nous reste encore à prier nos distingués compatriotes, MLII. Pierre Marty, Pagès-Allary, Lauby, Rieuf et P. Maury, d'agréer l'expression de notre cordiale gratitude pour les documents précieux que nous devons à leur inépuisable amabilité.

Maison de retraite de Montferrand (Puy-de-Dôme), 15 décembre 1908.

$$
\text { F. Héribaud Josepit. }
$$




\section{LES \\ DIATOMEES FOSSILES \\ D'A UVERGNE}

\section{DÉPOTS ÉTUDIÉS}

Les dépôts étudiés pour ce troisième Mémoire sont, pour le département du Cantal :

$1^{\circ}$ Les dépôts inédits de La Garde, de Lugarde, de Recoules, de Fontillou et d'Allanche.

$2^{\circ}$ Des affleurements nouveaux des dépôts déjà connus de Moissac, de Fraisse-Bas, de Celles et de Neussargues.

Pour le Puy-de-Dôme, nous avons laissé à notre distingué compatriote, M. Lauby, le soin de publier prochainement, à l'occasion de sa thèse inaugurale, les dépôts inédits et les affleurements nouveaux découverts depuis le mois de mai 1903. En ce qui concerne ce département nous nous bornerons à publier la florule du dépôt du Chambon, découvert par l'abbé Boulay, il y a une dizaine d'années.

A titre de documents pour la flore diatomique du Massif Central, nous donnerons le résultat de l'étude des dépôts d'Araules près d'Yssingeaux, de Mardansou près de Langeac, et d'Andreugeolet près de Saugues, découverts par M. Gallaud. 


\title{
I \\ DÉPOTS DU CANTAL
}

\author{
DÉPO'T DE LA GARDE
}

La découverte de ce dépôt a été faite en 1905, par II. Naury, Institutear; il est situé à 1050 mètres d'altitude, sur le flanc gauche de la haute vallée de la Véronne, à 2 kilomètres en aval de Colandre, canton de Riom-ès- Montagnes.

A 400 mètres en aval il existe un autre affleurement situé dans le lit même du ruisseau de Lagout, mais nous l'avons trouvé tout à fait semblable à celui de La Garde dont il est le prolongement.

En 1906 nons avons publié une première étude de ce dépôt, dans le mémoire de II. Maury sur les alluvions miocènes et pliocènes de la haute vallée de la V'eronne; les échantillons examinés ne provenant que d'un seul point dı dépôt, nous n'aviors pu domner qu'une liste incomplète; aujourd'luui, grâce à un envoi de spécimens nombreux et variés, reçus de MI. Maury, il nous est possible de compléter la florule diatomique de ce beau dépôt, avec les figures des espèces nouvelles observées.

D'après M. Maury, les argiles à Diatomées et à feuilles fossiles, se presentent en blocs interstratifiés dans des tufs andésitiques, surmontés par des formations successives de cailloux roulés, de conglomérat andésitique, de cinérites, et le tout couronné par une puissante traînée de basalte pliocène. 
Le dépôt de La Garde est très analogue à celui de Joursac, et la date de son remaniement, qui est celle de sa florule phanéroganique, appartient lien, comme Joursac au Niocène supérieur; d'ailleurs, M. Maury est arrivé au même résultat, même en se basant sur des considérations d'ordre différent : "Je n'ai rien à ajouter, dit-il, aux conclusions du Frère Héribaud sur la florule diatomique de La Garde qui nous fait connaître huit espèces nouvelles. Je suis heureux de constater que, par des roies différentes, nous arrivons au même rísultat pour fixer l'âge de ces argiles. En comparant la flore de ce dépôt avec celles de Joursac, de Neussargues, de Moissac et d'Andelat, le Frère Héribaud établit le sỵnchronisme de ces dépôts. De mon côté par des comparaisons lithologiques et stratigraphiques avec Jonrsac, et en me basant sur les données générales de la climatologie pontienne, je montre que les argiles à Diatomées de La Garde et du ruisseau de Lagout datent de cette époque $)^{(1)}$.

La florule phanérogamique de La Garde, établie par II. Maury, compte 18 formes, dont 14 ont été déterminées spécifiquement, et 4 n'ont pu l'être que génériquement, appartenant aux genres Acer, Celtis, Lxurus et Clmus.

Voici les espèces de cette florule:

Aspidium Filix-mas var. Salix cinerea var. auricupliocenicum Sap.

Trichomanes asplenüforme Sap. ?

Carex maxima Scop. late nov.

Alnus glutinosa var. fossilis Laur.

Carpinus suborientutis s.p.

(1) Loc. cit., p. 43. 
Corylus Arellana L.

Carya minor Sap.

Populus balsamoides fimp.

- alba L.

Fagus pliocenica Sap.

Zelliova Ungeri (Ett.) Kov.

Fraxinus arvernensis Laur

Hedera Helix L.

Parmi ces 14 espèces, 7 se retrouvent à Joursac, et les autres, sauf Populus alba, qui est noureau pour le Cantal, sont connues dans les dépôts de la Mougudo, Saint-Vincent, Niac, etc.; c'est donc avec Joursac que La Garde présente le plus d'analogie.

$\mathrm{Si}$ nous comparons maintenant la flore diatomique du même dépôt avec celles de Joursac, de Neussargues, Moissac et Andelat, dépôts du Miocène supérieur, ainsi que nous l'avons démontré dans le second Mémoire, nous trouvons que La Garde contient 27 espèces sur $\check{5}(1$ qui lui sont communes avec Joursac, 19 avec Moissac, 11 avec Neussargues et 10 avec Andelat. Le synchronisme de ces dépôts est donc hien établi.

L'examen des échantillons reçus de M. Maury nous a donné le résultat suivant :

Cocconeis lineata Grun. (Diat. d'Aur., p. 44).

- _ var. euglypte Girun.(Diat.d'Auv.,p. 4i)

- - - var. rotunda (Diat. foss. II, p. 5).

-- - Placentula Ehrb.( Diat. d’ Auv.,p. 44)

Coscinodiscus pygmeus var. micropunctata (Diat. d'Auv., p. 194).

Cymatopleura Solea Breb. var. apiculata Pritch.

(Diat. d'Auv., p. 161).

Cymbella Foucaudi F. Hérib. (Diat. foss., II, p. 16).

- cymbiformis Ehrb (Diat. d'Auv., p. 69).

- gastroides Ktz. (Diat. d'Auv., p. 68).

- Tevis N'eg. (Diat. d'Auv., p. 66).

- Laulyji F. Hérib. (Diat. foss. II, p. 18).

- lanceoluta Ehrb. (Diat. d'Auv., p. 68). 
Cymbella leptoceras Ktz. (Diat. d'Aur, p. 66). parva W. Sin. (Diat. d'Aux., p. 70).

Cymbella pseudo-turgidula sp. nov. (Pl. XIII, fig. 14) - Espèce de petite taille; longueur $35 \mu$, largeur $9 \mu$; face dorsale régulièrement courbée, face ventrale légèrement concave ou gilubeuse, extrémités coniques-arrondies ; raphé régulièrement arqué, ¿̀ pores centraux droits et non élargis, serrés de près par les stries; aires axiale et centrale nulles; stries régulièrement rayonnantes, non distinctement granulées, au nombre de 8 en $10 \mu$ au milieu de la face dorsale, de 11 en $10 \mu$ au milieu de la face ventrale, un peu plus serrées vers les extrêmités.

Cette espèce présente la structure du Cymbella turgiclula (A. Sch., Atl., pl. 9, fig. 23 et 26), Diatomée sanmâtre et récente décrite par Grunow, mais l'espèce de La Garde est plus grêle, ses extrêmités non atténuées et la striation différente.

Cymbella cantalense sp. nov. (Pl. XIII, fig. 17). - Longueur 45 à $50 \mu$, largeur 6 à $10 \mu$, face dorsale légèrement et uniformément courbée, face ventrale faiblement concave et légèrement gibbeuse au milieu, extrêmités laryement arrondies et non récurvées; raphé délicat, biarqué, à nodules peu marqués, les pores terminaux très légèrement récurvés et placés contre la face dorsale, à peu de distance des extrêmités de la valve; aire axiale notable, augmentant de largeur des extrêmités au milieu, où elle n'est pas arrondie autour du nodule médian; stries rayonnantes, indistinctement granulées, au nombre de 9 en $10 \mu$ à la partie dorsale et de $10^{1} \%$ en $10 \mu$ à la partie ventrale.

C'ette espèce a la physionomie générale du C'ymbella parea (T. II. Syn., pl. 2, fig. 14), mais elle s'en dis- 
tingue nettement par la forme de ses nodules et de l'aire axiale; se rapproche aussi du Cymbella Staubii Pant. Pant., Ung. III, pl. 8, fig. 131), mais le nôtre en diffère par sa forme plus élancée, et par ses stries beaucoup plus rapprochées.

Eneyonema ccespitosum Rab. (Diat. d'Aur., p. 73). Epithemiu cistula (Ehrb.) Pant. (Diat. Ung., III). - - var. lunaris (Ehrb.) Pant.

Eunotia pectinalis Rab. (Diat. d'Auv., p. 132). - - var. minor Ral.

Eunotia pendens sp.nov. (Pl. XIV, fig. 3). Largeur 13 à $14 \mu$, valve ì hords parallèles arqués, et dont la courbure augmente brusquement vers les extrêmités, qui sont un peu aplaties, largement arrondies et paraissent ainsi pendre; nodule terminal grand et bien visible; stries rayonnantes, non distinetement granulées ou divisées en travers, a u nombre de 9 à 10 en $10 \mu$, plus serrées aux extrêmités, où elles sont progressivement de plus en plus courbées pour entourer le nodule.

Se distingue de l'Eunotia parallela Ehrb. et de l'Eunotir Ehrenbergï Pant., par la forme et le nombre des stries, et de l'E. hungarica Pant., par la striation non ponctuée.

Fragilaria construens Grun. (Diat. d'Auv.,p. 143).

- _ - var. Venter Girn.(Diat. d'Auv., p. 14i).

- _ - var.pumila Grun.(Diat. d'Auv.), p. 153)

- brevistriata Grun. var. Mormorum Grun. (Diat. d'Auv., p. 147).

- Zeilleri F. Hérib. (Diat. foss. II, p. 26).

Gomphonema acuminatum Ehrb. (Thit. d'Aue', p. 53).

- - var. coronata Ehrb. (D. d’Auv., p. 54).

- _ var. intermedium Grun. (D.d'Auv.,p. sí) 
Gomphonema acuminatum rar. minutissima nov. (I'l. XIII, fig. 26). - Encore plus petite que la variété pusilla (V. H. Syn. pl. 23, fig. 19); atteint à peine $20 \mu$ de longueur: diffère en outre de la variété pusilla par la tête sensiblement plu; large que la partie centrale de la valve; stries an nombre de 13 à 14 en $10 \mu$.

Cette petite forme, lien distincte, est très commune dans l'échantillon étudié sous le n5.

Gomphonema Pisciculus $s_{p}$. nov. (Pl. XIII, fig. 24). - Très petit; longueur 15 à $20 \mu$, partie inférieure assez large et baciliaire, partie médiane dilatée, partie supérieure portant un étranglement et une partie capitée lancéolée, moins élargie que la partie médiane; stries au nombre de 15 en $10 \mu$.

La forme générale de cette espèce rappelle celle d'un petit poisson, d'où son nom spécifique de Pisciculus; assez fréquent dans le dépît, mais moins abondant que la variété précédente.

Gomphonema perfidum sp. nov. (I'l. XIII, fig. 28). - Très petit; longueur 10 ì $16 \mu$, valve trìs dilatée à la partie submédiane, avec une extrêmité supérieure retrécie et finement acuminée; stries au nombre de 14 à 15 en $10 \mu$, les médianes courbes, divergentes et plus écartées que celles des extrêmités, quelquefois fortement marquées jusque vers le milieu de la valve, où elles limitent alors une aréa bien nette, lancéolée, au milieu de laquelle on n'apercoit qu'un nodule central faible et pas de raphé; d'autres fois les stries se terminent moins nettement au milieu de la valve, l'aréa est plus étroite, linéaire, le nodule est plus visible, mais le raphé est toujours très peu visible ; c'est pourquoi nous avions classé primitivement cette Diatomée minuscule 
dans le genre Rhoicosphenia, mais n'ayant pu trouver, ni une valve isolée absolument dépourvue de nodule, ni une face connective courbée et ne présentant de nodule que d'un seul côté, nous avons dû la placer dans le genre Gomplionema.

Cette petite espèce se distingue nettement du Gomphonema acuminatum var. laticeps (V. H. Syn., pl. 23, fig. 18), dont la partie submédiane est angulaire, au lieu d'être arrondie, et l'extrêmité supérieure est plus robuste, non atténuée, contrairement à la Diatomée du Cantal.

On trouve assez souvent cette espèce déformée, avec un côté de la valve moins large et un contour moins fortement accusé que l'autre.

Gomplonema subclavatum Grun. (Diat.d'Auv.,p.55). - intricatum Ktz. (Diat. d'Aw., p. 57 ).

Gomphonema fossile sp. nov. (P'l. XIII, fig. 25). - Valve lancéolée, biconique, ì extrêmités largement arrondies, longueur 35 ì $40 \mu$; raphé et point unilatéral bien visibles; aréa lancéolée, bien définie et arrondie autour du nodule médian; stries très nettes, non distinctement granulées, rayonnantes, au nombre de 9 à 10 en $10 \mu$ au milieu, plus serrées aux extrêmités.

Diffère du Comphonema ventricosum par sa forme générale qui est moins élargie au milien, et contractée entre la partie médiane et l'extrémité inférieure, ainsi que par sa striation moins serrée; se distingue du Gomphonema commutatum, par son aréa plus grande et élargie autour du nodule médian.

Melosira lineolata var. robusta (Diat. foss. II, p. 77). - minuta F. Hérib. (Diat. foss. II, p. 32).

Melosira Mauryana sp. nov. (Pl. XIV, fig. 28 
et 29). - Diamètre des valves 55 à $70 \mu$; valve à centre très grand; côtes tout à fait marginales, ayant à peine f a $7 \mu$ de longueur, assez peu marquées, non terminées vers l'extérieur par une perle visible, an nombre de 8 à 9 en $10 \mu$; ̀̀ la surface intérieure, on aperçoit une zone de même largeur que celle qui est couverte par les stries, qui porte me ponctuation en quinconce de points très fins; sur l'une des valyes, cette ponctuation forme des lignes de stries décussées assez visibles, et sur l'autre des stries radiantes très fines mais visibles; on aperçoit en outre une couronne de gros granules placée à l'iutérieur de l'amneau valvaire. - Zone connective peu développée, d'une hauteur de 15 à $18 \mu$ pour chaque valve, couverte de points décussés, formant des stries croisées au nombre de 15 en $10 \mu$, et des stries longitudinales moins visibles, au nombre de 18 en $10 \mu$. Les côtes de la valve se présentent sous la forme de petites perles brillantes bordant la partie supérieure du frustule. A l'intérieur se trouvent deux lignes de granules peu visibles, dont l'écartement est d'environ $7 \mu$ dans tons les sens.

Se distingue du Melosira varennarum par ses stries moins fortes et non terminées par une perle, par la striation décussée visible sous les stries, par les granules placés à l'intérieur de l'anneau valvaire, et par la striation différente de la face connective.

Constaté assez commun dans $n^{0} 1$ et rare dans les autres échantillons.

Cette belle espèce, très distincte, est dédiée à II. Pierre Maury, Instituteur, à qui nous devons la découverte du dépôt de La Garde, et les échantillons étudiés.

Melosira undulata var. debilis (Diat. foss. II, p. 32).

- var. producta 1. Sct. (Diat. foss. I, p. 22) 
Navicula amphibola var, stauroneiformis M. P. et F. H. (Diat. foss. I, p. 37).

- acrosphceria var. barleana (D. foss. I, p. 21).

- Brauniu var. moissacensis (Diat. foss. II, p.63).

- Corbieri F. Hérib. (Diat. foss. II, p. 7 ).

- gastrum Donk. (Diat.d'Auc., p. 102).

- viridula Ktz. (Diat. d'Auv., p. 101).

- major Ktz. (Diat. d'Auv., p. 102).

- $\quad$ - var.convergentissima (D.at. foss. II, p. 10)

- peregrina var. fossitis (Diat. foss. II, p. 79).

- Placentula Ehrb. (Diat. d'Auv., p. 102).

- psendo-bacillum Grun. (Diat. d'Auv., p. 118).

- I'npula var. major. F. II. (Diat. foss. II, p. 39).

- radiosa var. acuta Grum. (Diat. d'Auv., p. 100).

Opephora Martyji F. Hérib. (Diat. foss. I, p. 3). cantalense var. olitusa (Diat. foss. II, p. 14).

Opephora Glangeaudi $s$. nne. (Pl. XIV, fig. 17 et 18). - Grande et belle forme, atteignant $60 \mu$ de longueur, peu dissymétrique, à partie médiane renflée; extrémités prolongées et quelquefois légìrement élargies, la supérieure faiblement tronquée-arrondie, l'inférieure régulièrement arrondie; côtes lisses, au nombre de 4 en $10 \mu$ au milieu, plus serrées aux extrêmités (5 à 6 en $10 \mu$ ), radiantes au centre, où elles sont parfois élargies vers les bords de la valve, radiantes et convergentes aux extrêmités, laissant au milieu de la valve une aréa longitudinale lancéolée plus ou moins dilatée au milieu. - Face connective peu conique.

Pourrait être rapproché de l'Opeploña Martyji var. robusta du dépôt d'Andelat (Diat. foss. II, p. 41), mais il s'en distingue nettement par sa forme et par ses côtès qui sont lisses, tandis qu'elles sont crénelées dans Opephora Martyi et sa variété rolusta.

Nous dédions cette espèce très distincte à M. Glan- 
geaud, l'éminent professeur de géologie à l'Université de Clemont, et nons le prions de l'agréer, comme un faible témoignage de notre reconnaissance pour l'intérêt qu'il veut bien prendre à nos recherches sur les Diatomées fossiles du Massif Central.

Nous avons constaté l'Opephora Glangeaudi commun daus l'échantillon $\mathrm{n}^{\circ} 2$ et rare dans les autres.

Stauroncis gracilis IV. Sm. (Diat. d'Auv., p. 76).

- Phenicenteron Ehrb. (Diat. d'Auv., p. 75).

Synedra capitata Ehrb. (Diat. d'Auv., p. 139).

- delicatissima IV. Sm. (Diat. d'Auv., p. 140).

- Ulna Ehrb. (Diat. d'Auv., p. 137).

Tabellaria flocculosa Ktz. (Diat. d'Aw., p. 155).

Van-Ileurckia rhomboides Bréb. (Diat.d'Auv.,p.110).

Avec les espèces nouvelles pour la flore générale, trouvées dans le dépôt de La Garde, les diatomistes remarqueront sur notre liste deux formes anciennes non mentionnées encore dans nos précédents Mémoires, ce sont: Epithemia cistula et sa var. lunaris; mais il est possible que ces deux Diatomées aient déjà été rencontrées et désignées sous les noms d'Epithemia gibberula et sa var. prorlucta, qui ont beaucoup d'analogie avec Epithemia cistula et sa variété lunaris, et que l'on pourrait considérer comme les formes récentes des deux formes fossiles.

Ehrenberg avait désigné cette espèce sous le nom d'Eunotia cistula, mais Kutzin et Ralls ne l'ayant pas mentionnée, elle était tombée dans l'oubli et avait disparu de la, nomenclalure. C'est Pentocseck qui a repris l'espèce et sa variété; il les a décrites et figurées dans son volume III des Diatomées de IIongrie, ce qui nous a permis d'identifier les deux formes fossiles de Hongrie avec celles des dépôts du Cantal. 


\section{DÉPOT DE LUGARDE}

Le dépôt de Lugarde a été découvert en 1906 par II. Rieuf, Conducteur des Ponts et Chaussées; il est. situé sur la ligne de Neussargues à Bort, à l'altitude de $1.013^{\mathrm{m}}$ et à $600^{\mathrm{m}}$ au S.-E. de la station de Lugarde, canton de Marcenat.

Notre savant compatriote, M. Pierre Marty, dont les travaux remarquables sur la flore fossile du Cantal sont bien connus des paléobotanistes, a relevé la coupe suivante concernant la stratigraphie du dépôt ${ }^{(1)}$.

$4^{\circ}$ Glacière, à gros blocs erratiques de basalte et de granite. Puissance : environ $2^{\mathrm{m}}$.

$3^{\circ}$ Alluvions sensiblement horizontales, argiles rousses, fevilletées, à empreintes végétales; schistes blanes à Diatomées, lignite, bois silicifié et rognons de pyrite de fer. Puissance : environ $t^{\mathrm{m}}$.

$2^{\mathrm{n}}$ Basalte très feldspathique. Puissance : environ $5^{\mathrm{m}}$.

$1^{\circ}$ Gineiss normal, perdant à $45^{\circ}$ vers le N.-O

Les argiles feuilletées à empreintes végétales contiennent aussi des Diatomées, mais elles sont moins riches que les schistes blancs, dans lesquels nous avons trouvé à peu près la totalité des espèces de notre liste.

Parmi les empreintes végétales, M. Marty a reconnu:

Jibocedrus salicornioides tg.

Typha latissima Al. Br.

Myrica lignitum Ung.

Planera Ungeri Kov.

Cinnamomum Scheuchaeri ls.
Trapa borealis Heer. Gladitschia allemanica $\mathrm{Hr}$. Robinia areernensis Laur. Parrotia pristina Ett.

(1) Note a l'Académie des Sciences, séance du 17 août 1908 . 
Avec ces 9 espèces bien déterminées, M. Marty a recueilli plusieurs formes qui n'ont pu recevoir d'attribution spécifique; elles appartiennent aux genres Marsilia, Equisetum, Populus, Ulmus et Fraxinus.

M. Marty fait observer que les denx espèces dominantes de Lugarde: Myrica lignitum et Cinnamomum Scheuchzeri, ont leur principale expansion an Miocène moyen, mais que la flore de Lugarde, comparée à la flore tertiaire en général, so rattache nettement au Miocène supérieur, tant par son ensemble que par ses espèces les plus abondantes et les plus caratéristiques; sur les 14 formes de la florule de Lugarde, 8 se retrouvent en effet dans le Miocène supérieur de Joursac; c'est donc avec la florule phanérogamique de ce dépôt que celle de Lugarde montre le plus d'affinité. Et comme les argiles qui contiennent cette florule reconvrent la coulée de basalte de Lugarde, il en résulte que ce basalte est non un basalte du Pliocène supérieur à Elephas meridionalis, comme on l'avait cru jusqu'ici, mais un basalte du Miocène supérieur ì Hipparion gracile.

Les conclusions de notre éminent compatriote sont d'ailleurs pleinement confirmées par la florule diatomique de Lugarde.

Nous avons établi cette florule d'après l'étude de matériaux volumineux reçus de MII. Marty et Rieuf; tous les échantillons examinés sont à peu près identiques, et caractérisés par la grande abondance du Staurosira Grunorï Pant.et d'un Ilelosira non moins abondant, et que notis n'arons pu identifier ì aucune forme connue.

Il est très curieux de retrouver à Lugarde le Stanroseris Grunowii, qui jusqu'ici était caractéristique des dépôts fossiles d'cau douce et saumâtre de IJongrie (Bory et Dubravica). Nous comparerons plus loin le 
dépôt du Cantal avec ceux de Hongrie, car ils contiennent en abondance plusieurs espèces communes, tout en étant cependant parfaitement distincts.

Le dépôt de Lugarde, comme tous les dépôts tertiaires du Cantal, dans lesquels on a trouvé des empreintes de feuilles, est un lambeau remanié, restratifié par les eaux et provenant du dépôt initial de Chambeuil; il se rattache à celui de Joursac, nun seulement par sa florule phanérogamique, ainsi que l'a démontré M. Marty, mais encore par les Diatomées caractéristiques communes aux deux dépôts, telles que: Achnanthes joursacense; Fragilaria nitida; Melosira undulata var. debilis; $\mathrm{Na}$ vicula Berriati, Nat. Costei et Nae. major var. convergentissima; l'identité des deux dépôts se trouve aussi confirmée par celle de leur complexe lithologique, dans lequel l'examen microscopique nous a permis de constater l'absence absolue de tout élément d'origine rolcanique, ce qui pronve bien que la formation de la masse diatomifère est antérieure aux manifestations du grand rolcan cantalien.

Toici la liste des espèces observées :

Achnanthes joursacense (Diat. foss., II, p. 5).

Anorthoneis Peragalli nov. (Pl. XIII, fig, 29 et 30 ).

Cocconeis lineata Grun. (Diat.d'Auv., p. 44).

\section{- var. euglypta Grin. (D. d'Auv., p. 46)}

Cymbella aspera Ehrrb. (Thiat. d'Auv., I, p. 69).

- cymbiformis Ehrb. (Diat. d'Aue., p. 64).

- gastroides Ktz. (Diat. d'Auv., p. 68).

- $\quad$ - $\quad$ var.minor. V.H.(D.d'Aue, p. 68).

- lanceolata Ehrb. (Thiat. l'A Aur., 1. 68).

- leptoceras Ktz. (Diat. d'Aur., p. 66).

Epithemia cistula Ehrb.) Pant. (Ihat. C'ng. III.) 
Epithemia cistula var. lunaris (Ehrb.) Pant.

- gibba Ktz (Diat d'Auv., 1). 126).

- - var.parallela Girn. (D. d'Auv., p. 126).

- - var. ventricosa Giru. (D.d'Auc., p.126)

- gibberula Elnb.'ar.producta Ginn.( I).d'Auv.,p. 129)

- Hyndmannii II. Sm. var. perlonga (D.f., II, p. 26)

- Sorex Ktz. (Diat. d'Auv., p. 126).

Eunotiagracilis Ehrb. (Diat. d'Aur., p. 131).

- parallela Ehrb. (Diat d'Aur., p. 133 ).

- pectinalis Ktz. (Diat.d'Auv., p. 132).

- - var, minor Rab.

- provupta Ehrb. (Diat d'Auv., p. 134).

Fragilaria nitida (Diat. foss., II, p. 127).

Fragilaria nitida var. curta nov. - Diffère du type par sa longueur beaucoup moindre, tout en conservant sensiblement la même largeur; longueur 40 i $50 \mu$, au lieu de 50 à $100 \mu$, comme dans la forme type de Joursac.

Gomphonema commutatum Grun. (Diat. d’.1ur., p. ว̆s.s). - intricatum Ktz. (Diat. d'Auv. p. 57 )

Melosira gramulata Ehrb. var. australiensis V. H. (Diat. foss. II, p. 34.)

- undulata Ktz. (Diat. foss. I, p. 22).

- - - var. debilis (Diat.foss., II, 1. 32).

-- $\quad$ - var. producta (Diat.foss., I, p. -2).

- varennarum (Diat. d'Auv., p. 189.

Melosira Rieufii F. Hérib. (Pl. XIV, fig. 27).Très robuste, formant des chaînes solides et assez longues. Très variahle comme diamètre, de 30 à $40 \mu$, ‘̀ frustules relativement courts, et dont la hanteur, moins variable que la largeur, est de 13 a $16 \mu$; face valvaire i 
centre grand et à stries marginales assez courtes, ayant 5 à $6 \mu$ de longueur, non terminées par une perle à l'extérieur; les stries, étroites et fortes an bord de la valve, s'élargissent et diminuent d'intensité de la périphérie au centre, et sont pour ainsi dire coniques, et au nombre de 6 ¿ 7 en $10 \mu$ au bord; face connective présentant des stries croisćes, au nombre de 21 en $10 \mu$ dans le sens longitudinal et de 18 en $10 \mu$ en diagonale; face de réunion des frustules étruite, crénelée, peu visible.

Se distingue des Melorisa Boulayana et rarennarum par ses stries non terminées par une perle, de forme différente et de longueur moindre, par le centre valvaire non creusé, et par la similitude des deux valves. Diffère du Melosira Mauryana, du dépôt de La Garde, par l'absence de striation intérieure, par le plus grand écartement des stries, par leur forme différente et par la striation de la face connective.

Cette espèce, très distincte, est dédiée ì MI. Rieuf, à qui nous devons la découverte du beau dépôt de Lugarde, où elle est très abondante.

Navicula amphibola Cl. var. stauroneiformis (Diat. foss., I, p. 37).

- Berriati F. Hérib. (Diat. foss., II, p. 37).

Cette belle espèce se rencontre à Lugarde sous des formes plus grandes qu'ì Joursac; leur longueur pent atteindre jusqu'à $35 \mu$.

Nacicula Berriati var. minor (Diat. foss., II, p. 14).

Navicula Berriati var. splendida F. Hérib. Pl. XIII, fig. 23). - Grande et belle forme; longueur $35 \mu$, largeur $21 \mu$; se distingue du type de Joursac par ses stries très élégamment et fortement courbées en sens contraire de celles du type an milieu 
de la valve, où elles laissent une aréa circulaire notable autour du nodule médian.

Naricula borealis Ktz. (Diat d'Auv., p. 86).

- Costei F. Hérib. (Diat. foss., II, p. 9).

- dicergens IV.Sm. (Diat. d'Auv., p. 89).

- gastrum Donk. (Diat. d'Awv., p. 109).

- limosa Ktz. (Diat. d'Auv., 111).

- major Ktz. (Diat. d'Auv., p. 82).

-... - var. convergentissima (D.f., II, p. 10).

- nobilis Ehrb. (Diat. d'Auv., p. 80).

- ventricosa Donk. (Diat. d'Auv., p. 113).

Opephora cantalense var. obesa nov. (Pl. XIV, fig. 19). - Trìs analogue à la variété capitata (Diat. foss., II, pl. X, fig. 15), mais s'en distingue par sa partie centrale toujours heaucoup plus dilatée que la partie supérieure, et qui n'en est pas séparée par un étranglement. Longueur 20 à $40 \mu$.

Fragilaria (Stanrosira) Harrisonï Grun. var. major (Diat.foss. II, p. 25).

Staurosira Grunowii Pant. (Pant. Ung. III, pl. 4, fig. 66). - Très abondant dans le dépôt, dont il constitue l'espèce caractéristique; de grandeur très variable, longueur du plus grand diamètre 25 à $55 \mu$; de forme également très variable, depuis des côtes peu concaves, surtout dans les petites formes, jusqu'ì des côtes profondément concaves vers le centre, principalement chez les grandes formes, avec des extrémités largement arrondies; les côtes sont au nombre de 4 ì 5 en $10 \mu$,

Staurosira Grunowii var. minor nov. (Pl. 
XIV, fig. 13). - De même forme que le type, mais beaucoup plus petite; longueur 20 ̀̀ $25 \mu$; côtes moins concaves; de forme parfois presque carrée, avec des côtes très faiblement courbes ; face connective relativement plus haute que dans le type.

Staurosira Grunowii var. major nov. (Pl. XIV, fig. 14). - Grande forme dont la longueur atteint jusqu'à $55 \mu$.

\section{Staurosira Grunowii var. triangulata} not. (Pl. XIV, fig. 15). - Forme anormale avec un angle a trophić beaucoup moins proéminent que les trois autres, et à l'intérieur duquel il n'y a pas de psendoraphé.

Staurosira Grunowii var. biangulata not. (Pl. XIY, fig. 16). - Forme anormale n'ayant plus que deux angles, et tout à fait semblable an Fragi?aria (Staurosira) Ilarrisonä var. major du dépôt de Joursac (Diat. foss., II, Pl. X, fig. 12).

Ces formes établissent le passage entre les Staurosira (Fragilaria) Harriconii et Grunowii, montrant ainsi les liens étroits qui réunissent ces deux espèces ; d'ailleurs Grunow avait admis pour cette espèce le nom de Stanrosira Harrisonii var. amplitetras.

S'ynedra L'nna Ehrb. (Diat. d'Auv., p. 137).

Tetracyclus Glans (Elhrl.) var. paucicostata nov. (Pl. XIV, fig. 20 et 21). - Diffère du Biblarium Glans Elırb. par le petit nombre de: côtes de la valve; elles sont parfois réduites à quatre, limitant les parties larges et les parties étroites de la valve; les courbures des côtes sont en sens contraire deux à deux (fig. 21), quelquefois une cinquième côte rectiligne et 
médiane, divise en deux la partie médiane élargie de la valve (fig. 20). La longueur varie entre 30 et $40 \mu$.

Le Tetracyclus Glans ne diffère du Tetracychus stella Ehrb. que par le développement moindre de la partie médiane, à peine plus large que les extrémités ; celles ci sont plus capitées, la constriction intermédiaire étant plus forte.

La Tetracyclus du dépôt de Celles, décrit et figuré dans le Mémoire de 1902, page 17, pl VIII, fig. 9, n'est pas tout ì fait conforme aux figures du Tetracyclus stella données par Ehrenberg, et doit être considéré comme une forme intermédiaire entre les T'etracyclus stella et Glans.

La présence en grande abondance du Staurosira Grunow ii, dans le dépôt de Lugarde, espèce qui n'était encore connue, d'après Pantocseck qu'en Hongrie, notamment dans le dépôt de Bory, est très remarquable, et conduit naturellement à comparer ces deux dépôts.

Voici la liste des Diatomées constatées, par le commandant H. Peragallo, dans un échantillon du dépôt de Bory, et publiée sous le $n^{\circ} 509$, dans les séries des Diatomies du momle entier, par 'Tempère et H. Peragallo; cette liste ne diffère pas notablement de celle que nous avons établie, d'après l'examen de deux préparations de Bory, reçues de M. P. Petit.

Achnanthes lanceolata. Cymbella austriaca.

- cymbiformis.

- gastroides rar. crassa.

- lanceolaia.

- leptoceras.

- Sturii.

Cocconeis limeater.
Epithemia cistula rar. lunaris Eunotia iucisa.

- minor.

- polyglyphis.

Melosira arewaria.

- cremulata.

- Rrseana. 
Navicula arata.

- Bacillum,

- dubravicensis.

- diphacela.

- elliptica var. Grandis.

- Hanerii.

- major.

- nobilis.
Navicula ientricosa.

Nitzsclia scalaris.

- Triblionella.

Staurosira Grunouii. intermedia.

Surirella Clementis.

- subsalsa.

Dans cette liste nous avons souligné les espèces communes aux deux dépòts, elles sont au nombre de 12 sur 31 et 52 formes constatées respectivement dans les dépôts de Bory et de Lugarde, et en supprimant les 16 rariétés de Lugarde, nous aurions 12 espèces communes entre 30 et 36 , ce qui fait plus d'un tiers d'espèces communes entre les deux dépôts, et, eu égard ì la fréquence et ì la particularité du Staurosira Grunouii et de l'Epithemia cistula var. Iunaris, il serait possible d'affirmer le synchronisme des deux dépôts.

Toutefois, nous ferons observer que le dépôt de Lugarde contient des espèces abondantes qui manquent absolument dans celui de Bory, comme Epithemia gibla, Taricula Berriati et Opephora cantalense: en revanche, le dépôt de Bory, possède Cymbella Sturï, Navicula Hanerï, Surirella Clementis et Nitzschia scalaris, espèces fréquentes qui manquent à Lugarde; les deux dépôts sont donc bien distincts par plusieurs espèces caractéristiques. 


\section{DÉPOT DE RECOULES}

Le dépôt de Recoules, découvert par notre éminent ami, M. l'agès-Allary, est situé sur le plateau de Recoules, entre le village de ce nom et le vaste dépôt de Joursac, à l'altitude d'environ 950 mètres.

Nous n'avons pu examiner encore ce dépôt sur place; dans les échantillons volumineux reçus de M. PagèsAllary, nous n'avons pas trouvé d'empreintes de fenilles, et si la masse diatomique en est absolument dépourvue, le dépôt de Recoules derrait être considéré comme un lambeau de projection non restractifié, provenant du dépôt initial de Chambeuil, mais, nous émettons ici une simple hypothèse, au sujet de laquelle nous aurons ì revenir plus tard; actuellement nous ne sommes pas suffisamment documenté pour élucider ce point spécial.

Les Diatomées de Recoules sont en général peu fragmentées, et presque toutes épiphytes; les espèces vaseuses ou pélagiques font à peu près complètement défaut. Les genres Epithemia-Cymbella, Nacicula et Fragilaria sont très largement représentés par de nombreux individus; les autres genres et leurs espèces ne le sont que par un très petit nombre de formes presque isolées.

La liste suivante a ćté établie d'après l'examen d'une douzaine d'échantillons :

Cocconeis lineata Ehrb. (Diat. d'Auv., p. 44).

- Placentula Ehrb. (Diat. d'Aus., p. 44). 
Cymbella austriaca, Grun. var. recta not: (Pl. XIII, fig. 10). - Peu dissymétrique; face ventrale légèrement conrexe, face dorsale faiblement angulaire, extrémités coniques, largement et obliquement tronquées-arrondies; raphé complexe, presque droit, à pores petits, ronds, les terminaux placés près des extrémités; aire axiale lancéolée, non élargie autour du nodule médian; stries granulées, parallèles an centre et devenant courergentes à mesure qu'elles se rapprochent des extrémités, au nombre de 8 en $10 \mu$ au milieu de la partie dorsale, et de 9 en $10 \mu$ au milieu de la partie ventrale, un peu plus serrées aux extrémités. Longueur de la value 60 à $70 \mu$, largeur 12 à $16 \mu$.

Ressemble à la figure du Cymbella austriaca Crun., de A. Sch. Atl., pl. 71, fig. 67, mais la forme de Recoules en diffère par presque tous ses détails.

Cymbella gallica rar. gracilis nor: (Pl. XIII, fig. 8). - Diffère du type par sa forme beaucoup plus grèle, par son aréa axiale plus étroite et non élargie autour du nodule médian du côté dorsal, et par ses nodules terminaux moins développés. Longueur de la valve $105 \mu$, large $16 \mu$; stries au nombre de 7 en $10 \mu$ du côté dorsal, et de 9 en $10 \mu$ du côté ventral.

Cymbella gallica var. minor nor. (Pl. XIII, fig. 9). - Se distingue du type par sa taille plus petite, par sa forme proportionnellement plus large et ses extrémités plus étroites. Longueur $65 \mu$, largeur $15 \mu$, 8 stries dorsales en $111 \mu$ et 9 ventrales au milieu de la valve.

Gymbella intermedia sp. nor. (Pl. XIII, fig. 15). - Intermédiaire entre le Cymbella pseudotriggidula et notre $C y m b e l l a$ miocenica; l'aire axiale est 
notable, mais moins large que dans la deuxième espèce; les stries, indistinctement granulées, sont an nombre de 8 en $10 \mu$, aussi bien du côté ventral que du côté dorsal, et légèrement plus serrées aux extrémités.

\section{Cymbella pseudo-turgidula var. dilatata} nov. (Pl. XIII, fig. 16). - Diffère du type par sa dilatation générale dans le sens transversal; par la face dorsale plus courbe, par la face ventrale plus gibbeuse; par l'aire axiale plus étroite que dans Cymbella intermerlia; forme intermédiaire entre Cymbella pseudoturgidula et Cymbella intermedia. Longueur de la valve 35 ¿ $45 \mu$, largeur de 10 ¿ $13 \mu ; 8$ stries dorsales et 9 ventrales en $10 \mu$.

Cymbella tenuis $s p$. nov. (Pl. XIII, fig. 11). De forme elliptique allongée, presque tout à fait symétrique, à extrémités légèrement produites et largement arrondies; raphé droit et très légèrement arqué; aire axiale très petite, lancéolée, arrondie autour du nodule médian; stries fines, non distinctement granulées, rayonnantes au centre de la valve, divergentes aux extrémités, plus écartées et plus fortes au milieu de la valve, où elles sont au nombre de 16 en $10 \mu$ au milieu du côté ventral, beaucoup plus serrées aux extrémités. Longueur de la valve 35 à $45 \mu$, largeur 12 i $13 \mu$.

Ressemble an Cymbella subcequalis Grun.( V.H. Syn., pl. III, fig. 2), mais notre espèce est moins élancée, plus symétrique et beaucoup plus finement striée.

Cymbella affinis Ktz. (Diat. d'Auv., p. 66).

- gastroides Ktz. (Diat. d'Auv, p. 68).

- heteropleura Grun.

- leptoreras Ktz. (Diat. d'Auv., p. 66).

- parra W. Sm. (Diat. d'Auv., p. 70).

- subcequalis Grun. (Diat. d'Awv., p.65). 
Encyonema lunula Grun. (Diat. d'Auv., p. 75). - ventricosum Grun. (Diat. d'Aur., p. 73). Epithemia gibba Ehrb. (Diat. d'Auv., p. 126).

- Hyndmannii W. Sm. (Diat. d'Auv., 1. 125).

- $\quad$ - var. perlonga (Diat. foss., II, p. 28)

- - var. curta F.H.(D.d'Auv., p.125).

- turgida Ktz. (Diat. d'Auv., p. 12t).

- - var. gramulata (D. d'Auv., p. 125).

- Westermannü Ktz. (Diat. d'Awe, p. 125).

Navicula bacilliformis Grun. (Diat. d'Auv., p. 117).

- Esox Ehrb. var. recta (Diat. foss., 1I, p. 11).

- major Ktz. (Diat. d'Aur., p. 82).

- mestris Ktz. (Diat. d'Auv., p. 84).

- sculpta Ehrb. (Diat.foss., I, p. 41).

- suluacuta Ehrb. (Diat d'Auv., p. 85).

- viridis Kरtz (Diat. d’Auv., p. 83).

Fragilaria brevistriata var. Mormorum Grun. (Diat. l'Aur., p. 147).

- construens Grun. (Diat. d'Auv., p. 143).

--- elliptica Schum. (Diat. d'Auv., p. 145).

- nitida (Diat. foss. II, p. 27).

- lapponica Grun. (V.H. Syn.pl.45, fig. 35).

Fragilaria lapponica var. gigantea nov. (Pl. X1V, fig. 9), - Ressemble au Fragilaria lapponica Grun., mais il est beaucoup plus grand, et les stries marginales sont plus écartées. Longueur 75 ̀̀ $85 \mu$, largeur 8 à $9 \mu$, stries au nombre de 8 en $10 \mu$.

Gomplionema commutatum Grun. (Diat.d'Aur., p. 55).

- intricatum Ktz. (Diat. d'Auv., p. 57).

- subclavatum Grun. (Diat.d'Auv., p. 55)

- $\quad$ - var. major nov. (Pl. XIII,

fig. 18). - Se distingue du type par sa longueur, qui peut atteindre jusqu'ì $65 \mu$. 
IIantzschice amplioxys Grun. var. xerophila Grun.

Melosira lavis Grun. (Diat. d'Aur., p. 187).

Nitzsclia sigmoidea (Diat. d'Alu., p. 167).

Stauroneis Phanicenteron Ehrb. (Diat. d'Aur., p. 75 ).

S!medra biceps Ktz. (Ilicrogéol.).

- capitata Ehrb. (Diat. d'Auv., p. 139).

Gyclotella Gharetoni var. gemmatula nox. (Pl. XVI, fig. 31). - - Se distingue du type par ses stries plus fortes, plus écartées, présentant à la base un renflement en forme de perle ovale; presque toutes les stries arrivent jusqu'ì l'aréa centrale, il n'en existe quelques-unes de plus courtes que dans les portions de la valve où les bords sont les plus fortement courbés, le contour de la valve étant ordinairement elliptique. Diamètre de 20 à $30 \mu$; stries au nombre de 10 en $10 \mu$ vers le bord de la valve. Cette forme intéressante est intermédiaire entre Cyclotella Charetoni et Cyclotella Kutzingiana var. gemmatula F. Hérib. 


\section{DÉPOT DE FONTILLOU}

Le dépôt de Fontillou est situé au Nord de Murat, près de la sortie de la ville, et à moins d'un kilomètre du dépôt de Faufouilhoux et de celui d'Auxillac.

Les échantillons étudiés nous ont livré les espèces suivantes:

Anorthoneis Peragalli sp. not. (Pl. XIII, fig. 29 et 30 ). - De forme presque orbiculaire, petite ; longueur 17 à $25 \mu$, largeur 16 à $21 \mu$; valve inférieure présentant la structure de celle du Corconeis lineata Ehrb., formée de stries rayonnantes, au nombre de 16 en $10 \mu$, dont les granules allongés sont disposés en lignes longitudinales ondulées; pseudo-raphé excentrique, étroit, allant jusqu'au bord de la valve. La seconde valve (fig. 29) nous a paru semblable ì la première, à l'exception dı raphé, lequel, d'ailleurs, est excessirement faible, et nous n'arons pu l'apercevoir distinctement.

$\mathrm{Au}$ premier abord, on pourrait prendre cette Diatomée pour une variation ronde du C'occoneis lineata. Un Cocconeis, en effet, étant courbé parallèlement au grand axe de la valve, et qui serait posé sur une des parties de la face valvaire, se présentant ainsi en vraie grandeur, aurait l'autre partie inclinée et se présentant en raccourci, serait vue plus étroite que l'autre; il en résulterait que le pseudo-raphé paraîtrait excentrique, et pourrait faire diagnostiquer un Anorthoneis; mais ici les exemplaires de l'espèce ne scnt pas très rares; ils sont 
de forme et de dimensions constantes, et différentes de celles des exemplaires du Cocconeis lineata, que l'on trouve également commun dans le dépôt, et chez tons les exemplaires nous avons constaté l'excentricité du pseudo-raphé. Nous avons même observé un frustule placé comme nous l'avons indiqué plus haut, c'est-à-dire que la moitié d'une valve entière était bien nettement visible à un même foyer de l'objectif, et le bord opposé était à un foyer sensiblement différent du premier, et ce bord était précisément celui qui était le plus éloigné du pseudo-raphé, ce qui aurait dû être le contraire si l'espèce avait été un Cocconeis.

Nous dédions cette jolie Diatomée à notre savant ami, le Commandant Maurice Peragallo, en reconnaissance des services nombreux qu'il nous a rendus au cours de nos études diatomiques.

Cocconeis lineata Grun. (Diat. d'Auv., p. 44).

Campylodiscus costatus W. Sm. (Diat.d'Auv, p. 182)

Coscinodiscus pygmaus (Diat. d'Aux., p. 194).

var. micropunctata (Diat.

$$
\text { d'Am., p. 194). }
$$

Cymatopleura Solea Bréb. (Diat. d'Auv., p. 161). - var. apiculata (W. Sm).

Cymbella affinis Ktz. (Diat. d'Auv., p. 66).

- aspera Ehrb. (Diat. d'Auv., p. 69).

- Brevieri F. Hérib. (Diat. foss. II, p. 16).

- cistula Hempr. (Diat. d' Auv., p. 70).

-- - var. fusidium (Diat. d'Auv., p. 71).

- conifera F. Hérib. (Diat. d'Auv., p. 220).

- cymbiformis Ehrb. (Diat. d'Auv., p. 69).

- lancenlata Ehrb. (Diat. d'Aur., p. 68.)

- parva IV. Sm. (Diut. d'Auv., p. 70).

- Pauli MI. Per. (Diut. d'Auv., p. 70). 
Enc?,onema prostratum Ralfs (Diat. d'Auv., p. 72).

- ventricosum Ktz. (Diat. d'Aur., p. 73).

Epithemia gibba Ehrb. (Diat. d'Aur., p. 126).

- - var. parallela (Diat. d'Auv., p. 126).

- gibberula var. producta (D.d'Auv., p.129.)

- Hyndmannii TV.Sm. (Diat. d'Aur., p.125).

- $\quad$ - $\quad$ var. perlonga (D.f. II, p. 28).

- Sorex Ktz. (Diat. d'Auv., p. 126).

- turgida Ktz. (Diat. d'Auv., p. 124).

- Westermannï Ktz. (Diat. d’Auv., p. 125).

- Zebra Ktz. (Diat. d'Aur., p. 127).

- - var. proboscidea (D.d'Auv., p. 128).

Eunotic polydentula Ehrb. (Diat. d'Auv., p. 134).

Fragilaria brevistriata Grun. (Diat. d'Aue., p. 146).

- construens Grun. (Diat. d'Aur., p. 143).

- elliptica Schum. (Diat. d'Auv., p. 145).

- lapponica Grun.(V. H. Syn. pl. 45, fig.35).

Gomplionema cantalicum F. Hér. (D. d'Auv., p. 219). - commutatum Gr. (Diat. d'Auv., p. 55).

- intricatum Ktz (Diat. d'Aur., p. 57).

Melosira varennarum. Per. et F. H. (I). d'Aux.,p.189).

Naricula acrospleceria Bréb. var. elongata F. H. et II. P. (Diat. foss. II, p. 64).

- elliptica Ktz. (Diat. d'Aur., p. 104).

- major Ḱtz. (Diat. d'Auv., p. 82).

- nobilis Ehrb. (Diat. d'Auv, p. 80).

-- radiosa Kitz. (Diat d'Aur., p. 99).

- Reinhardtii Grun. (Diat. d' Auv., p. 102).

Stauroneis Phomicentreon Ehrh. (Diat. d'Auv., p.75).

Surivella norvegica Ehrb. (I)iat. d'Auv., p. 176).

- roluesta Ehrb. (Diat. d'Aur., p. 180).

- tenera Gireg. (Jiat. d'Aur., p. 180. 
Surirella Brunhesi nov. sp. (Pl. XIT, fig. 12). - A. Schmidt (Atl., pl. 23, fig. 1) donne le dessin d'une forme voisine du Surivella lifrons Ktz. qui a bien quelque analogie avec le Surirella de Fontillou, mais le nôtre se distingue nettement de celui de Schmidt, Diatomée actuelle de l'Amérique du Sud, par sa taille toujours plus petite, par ses extrémités moins dissemblables, par les côtes moins obliques par rapport à la ligne médiane de la valve, et plus serrées $\left(2\right.$ ¿ $2 \frac{1}{2}$ en $10 \mu$, an lieu de $1^{1 / 4}$ ‘̀ $1 \frac{1}{2}$ en $10 \mu$ ); diffère du Surirella bifrons Ktz. par sa forme générale, par la striation et par la silice beaucoup plus délicate.

Longueur de la valve 100 à $115 \mu$, largeur 50 à $55 \mu$ Espèce très distincte et assez fréquente à Fontillou.

Nous dédions cette belle Diatomée à notre savant compatriote, M. Bernard Brunhes, professeur de physique à l'Université de Clermont et Directeur de l'Observatoire du Puy-de-Dôme.

En comparant la florule de Fontillon avec celle d'Auxillac, publiée en 1893, on constate qu'elles sont très analogues, et l'on est conduit à voir dans le dépôt de Fontillou un prolongement de celui d'Auxillac; d'ailleurs la fréquence du Gomphonema cantalicum dans les deux dépôts, en est une preuve concluante. 


\section{DÉPOT D'ALLANCHE}

Le dépôt d'Allanche, découvert par notre compatriote, II. Lauby, est un dépôt pélagique; il est presque exclusivement formé du C'yclotella perforata et de quelques Melorisa; les espèces épiphytes font presque complètement défaut et ne se trouvent, pour ainsi dire, qu'à l'état isolé.

Sa florule diatomique est très analogue avec celle de Moissac et de Joursac; sur les 26 espèces ou variétés observées 10 se trouvent à Moissac, et parmi elles 4 sont caractéristiques de ce dépôt; 10 espèces se trouvent aussi à Joursac, dont 3 caractéristiques.

En résumé, les espèces d'Allanche se retrouvent à Mloissac et ì Joursac, à l'exception de : Epithemia cistula (Ehrb.), Epithemia gibba var. centricosa, Melorisa tenuissima et Melosira strangulata, espèce caractéristique du dépôt et inédite.

Les 26 espèces ou variétés observées sont :

Cocconeis lineata var. euglypta Gir. (Diat. d'Auv., p. 46). Navirula Berriati var. minor (Diat. foss., II, p. 14). - pseudo-bacillum Grun.(Diat. d'Auv., p. 118).

- radiosa Kız. (Diut. d'Auv, p. 99).

- trianmulifera (Diat. foss., II, p. 9).

Amphora Borneti F. Hérib. (Diat. foss., II, p. 70).

Cymbella cuspilata Ktz. var. minor (Diat. foss.II.p. 91). Cette jolie forme n'était encore connue que dans le dépôt de Ceyssac (Haute-Loire). 
Cymbella cymbiformis Ehrb. (Diat. d'Aur., p. 69).

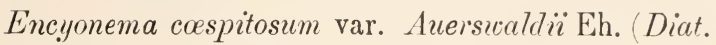

$$
\text { d'Auv., p. 73). }
$$

Gomphonema parrum (Diat. foss. II, p. 22).

Fragilaria Gustavei F. Hérib. (Diat. foss. II, p. 25).

-- minutissima Grun. (Diat. foss. II, p. 26).

- mutalitis Grun. (Diat. d'Auv., p. 145).

Epithemia cistula (Ehrb.) Pant. (Diat. Ung. III).

- gibba Ktz (Diat. d'A vv., p. 126).

- - var. ventricosa (Diat. d'Auv. p. 126).

- turgida Ktz. (Diat. d'Auv., p. 124).

Cymatopleura Martyi F. Hérib. (Diat.foss. II, p. 75).

Surirella bifrons Ehrb. (Diat. d'Auv., p. 177).

Opepleora cantalense F. Hérib. (Diat. foss., II, p. 30 ). - var. capiiata (Diat.foss. II, p. 30).

Melosira cremulata var. ambigua Gir. ( D. d'Auv., p. 187)).

- tenuissima Grun. (Diat. d’Auv., p. 188).

- undulata Ktz. var.producta (D. foss. I, p. 22)

Cyclotella perforata F. Hérib. (Diat. foss. II, p. 34).

Melorisa strangulata nov. $s p$. (Pl. XIV, fig. 25, 26). - Se distingue du Melorisa cremulata var. ambigua Grun. par son sillon beaucoup plus prononcé, rétrécissant considérablement l'ouverture de la valve, surtout dans les petits exemplaires. Stries granulées, au nombre de 16 ̀̀ 18 en $10 \mu$; diamètre de 5 à $10 \mu$; longueur du demi frustule de 8 à $15 \mu$. 


\section{DÉPOT DE MOISSAC}

Dans le Mémoire de 1903, page 57, nous arons donné le résultat de l'étude de deux affleurements du beau dépôt de Moissac, situés l'un et l'autre sur le flanc gauche de la vallée de Sainte-Anastasie, creusće par le ruisseau d'Allanche.

En 1904, M. Pagès-Allary nous annonẹa la découverte d'un dépôt à Diatomées, affleurant sur le talus de la nouvelle ligne de Neussargues à Bort, situé sur le flanc droit de la même vallée, et presque à la sortie du village de Moissac; au mois d'août de la même année, au cours d'une excursion au Cantal, nous êtmes le plaisir de visiter ce dépôt, en compagnie de notre distingué compatriote; ce lambeau remanié, peu volumineux, ayant à peine quelques mètres cubes, se trouve interstratifié au milieu de roches de nature très diverses, analogues à des accumulations morainiques; la masse diatomifère se détache nettement des roches englobantes, par sa couleur d'un gris clair, par sa densité très faible et par son complexe lithologique.

L'examen de six échantillons nous a donné le résultat suivant :

Achnanthes Flahaulti F. Hérib. (Diat.foss., II, p. 57).

Ampliora Borneti F. Hérib. (Diat. foss., II, p. 70 ).

Cyclotella perforata M. P. et F.H. (Diat. foss., II, p. 34).

Cymbella Brevieri F. Hérib. (Diat. foss., II, p. 16).

- Creguti F. Hérib. (Diat. foss., II, p. 17). 
Cymbella cistula Hemp. (Diat. d'Aur., p. 70 ).

- cuspidata Ktz. (Diat. d'Aur., p. 65).

- cymbiformis Ehrb. (Diat. d'Auv., p. 69).

Epithemia gillua Ehrb. (Diat. d'Auv., p. 126).

-- Ifyndmannii II. Sm.(Diat. d'Aur., p. 125).

- Sorex Ktz. (Diat. d’Auv., p. 126).

- Zebra Ktz. (Diat. d'Auv., p. 127).

Eunotia arverna sp. nov. (Pl. XIV, fig. 2). Légèrement arqué, face ventrale très faiblement et régulièrement concave, face dorsale régulièrement convexe, d'une courbure un peu plus forte que celle de la face opposée; extrémités légèrement produites dans la direction de la face ventrale, insensiblement capitées et coniques du côté dorsal, largement arrondies; nodules très gros, placés à l'extrémité de la valve; stries rayonnantes, trìs fines, indistinctement ponctuées, d'écartement variable, serrées au milieu ( 8 en $10 \mu$ ) et aux extrémités (9 à 10 en $10 \mu$ ), beaucoup plus écartées, dans les parties intermédiaires ( 5 à 6 en $10 \mu$ ); longueur de la valve $95 \mu$, largeur $13 \mu$.

Intermédiaire, comme forme, entre Eunotia monodon Elırb. et Eunotia major Ehrb., mais ressemblant plutôt au premier; il se distingue des deux espèces d'Ehrenberg par la striation, qui est tout à fait différente; dans l'E. monorlon les stries sont équidistantes et plus serrées $(12$ en $10 \mu)$ et dans l'E. major elles sont plus serrées aux extrémités qu'au milieu (11 à 12 en $10 \mu$ ).

Fragilaria moissacensis sp. nor. (Pl. XIV, fig. 5).- Bacıllaire, à partie médiane fortement élargrie, extrémités largement arrondies; stries radiantes et courbes jusque près des extrémités qu'elles n'atteignent pas tout à fait; pseudo-raphé étroit, mais bien apparent; 
lorgueur de la valve $36 \mu$, largeur au centre $10 \mu$, aux extrémités $6 \mu$; stries au nombre de $11 \frac{1}{2}$ en $10 \mu$.

Diffère d'une grande forme du Fragilaria Gustavei F. Hérib. (Diat. foss. I, pl. X, fig. 13), par son pseudoraphé bien défini, par ses stries plus écartées et bien marquées jusqu'au pseudo-raphé.

Gomphonema licmophoraoides (Diat. foss., II, p. 24).

Melosira cremulata var. ambigua (Diat. d'Auv., p. 187).

- tenuissima Grun. (Diat. d’Auv., p. 188).

- undulata Ktz.var. debilis (Diat.foss., II, p. 32).

Nacicula Berriati F. Hérib. (Diat. foss., II, p. 13).

- $\quad$ - var. minor $\mathrm{F}$. Hér. (D) foss., II, p. 14).

- gastrum Donk. (Diat. d'Auv., p. 102).

- major Ktz. (Diat. d'Auv., p. 82).

- pseudo-bacillum Grun. (Diat.d'Aur., p. 118).

- rarliosa Ketz. (Diat. d’Auv., p. 99).

Opephora cantalense F. Hérib. (Diat. foss., I I, p. 500).

- - var. capitata (Diat. foss., II, p. 30).

-- Martyi var. robusta F. H. (Diat.foss., II, 1. 41).

La physionomie de cette florule ressemble beaucoup à celle des deux premiers affleurements connus du dépôt de Moissac, et il est évident que celui que nous renons d'examiner n'est autre chose qu'un lambeau séparé aujourd'hui de la masse principale par le creusement de la vallée.

Ce troisième affleurement est surtout remarquable par la très grande aboudance du Melosira crenulata var. ambigua et du Cyclotella perforata.

A propos de cette dernière espèce nous avons constaté qu'elle diffère sensiblement de notre Cyclotella perforata type. (Diat. foss., II, pl. X, fig. 35), caractérisé par sa taille très petite, à valve épaisse, avec un trou 
très visible; ici, au contraire, les frustules sont en général plus grands à valve mince et délicate, le centre grand et sablé, le trou moins visible; ces différences, observées dans le Cyclotella de l'affleurement noureau de Moissac, en font une forme intermédiaire entre le Cyclotella Kutzingiana var. granulata et le Cyclotella perforata type, appartenant l'un et l'autre ì Joursac et à Moissac.

\section{DÉPOT DE FRAISSE-BAS}

Les échantillons examinés, reçus de M. PagèsAllary, proviennent d'un affleurement nouveau situé sur le talus de la route, à quelques centaines de mètres de la masse principale de ce dépôt initial, déjà étudié et publié en 1903.

Les espèces olsservées, et non mentionnées dans notre Mémoire, page 46, sont :

Navicula borealis Ktz. (Diat. d'Auv., p. 86).

- divergens W. Sm. (Diat. d'Aur., p. 89).

- gracilis Ehrb. (Diat. d'Auv., p. 99).

Eunotia divisa $s p$, nov. (Pl. XIV, fig. 4). Face dorsale régulièrement courbée, face ventrale rectiligne; extrémités infléchies vers la face ventrale, et largement arrondies; stries fines, non distinctement granulées, alternativement fortes et faibles, excepté vers les extrémités, oì elles sont toutes sensiblement de la même intensité. Longueur de la valve 63 à $65 \mu$ 
largeur 11 à $12 \mu$; stries 15 en $10 \mu$. Espèce très distincte.

Eunotia minor Rab. (Diat. foss., II, p. 122).

Fragilaria brevistriata Grun. (Diat. d'Auv., p. 146).

- construens Grun. (Diat. d'Auv., p. 143).

Melosira strangulata sp. nov. (Pl. XIV, fig. 25 et 26 ); bien conforme à celui du dépôt d'Allanche.

Stauroneis gracilis WV. Sm. (Diat. d'Auv., p. 76).

Opepliora cantalense F. Hérib. (Diut. foss., II, p. 30). Coscinodicus dispar, var. radiata (Diat.d'Auv.p. 194). Tetracyclus?...

N'ayant trouvé que le connectif, ce Tetracyclus n'a pu recevoir d'attribution spécifique.

\section{DÉPOT DE CELLES}

La florule diatomique du beau dépôt de Celles, situé près la gare de Neussargues, et actuellement exploité avec beaucoup d'intelligence et de succès, par son honorable propriétaire, M. Pagès-Allary, a été publiée dans le Mémoire de 1902 ; mais, cette première étude était restée incomplète par suite de documents iusuffisants.

Nous avons eu l'occasion de revoir ce vaste dépôt an mois de septembre 1907, etnous donnons ici le résultat de l'étude que nous arons faite des échantillons pris sur divers points de la masse diatomifère ; nous ne men- 
tionnons que les espèces non constatées dans les échantillons examinés en 1902.

Amphora gracilis Ehrb. (Diat. d'Auv., p. 63).

- ovalis Ktz. (Diat. d'Aur., p. 62).

Navicula gentilis Ehrb. (Diat. d'Auv., p. 81 ).

Stauromeis Plumicenteron Ehrb (Diat. d'Aur., 1. 75).

Epithemia gibba varr. ventricosa (Diat. d'Auv., 1. 126).

Melosira canalifera ( Diat. d'Aur., p. 224).

Coscinodicus Boulei M. Per. ( Diat. foss., II, p. 112).

Le Coscinorlicus Boulei n'était connu jusqu'ici que dans le dépôt du Monastier (Haute-Loire) et dans celui de Gourgouras (Ardèche); la découverte de cette belle espèce saumâtre, dans le dépôt de Celles, est d'un très grand intérêt, et vient à l'appui de l'opinion que nous avions émise sur l'analogie des dépôts tertiaires de la Haute-Loire et de l'A rdèche avec ceux du Cantal; nous sommes d'ailleurs persuadé que les recherches ultérieures confirmeront notre manière de voir concernant le synchronisme de ces dépôts.

\section{DÉPOT DE NEUSSARGUES}

L'étude de plusieurs échantillons provenant d'un aífleurement nouveau, situé entre la gare de Neussargues et l'Allagnon, ne nous a donné aucune forme nouvelle à ajouter aux résultats publiés successivement en 18!3, 1902 et 1903, mais nous avons constaté que les échan- 
tillons examinés contiennent en grande abondance Melorisa canalifera, et que Gomphonema cantalicum et Cymbella conifera, s'y trouvent aussi en assez grande quantité ; or, ces trois espèces étant caractéristiques du dépôt d'Auxillac, lui rattachent, en toute évidence, celui de Neussargues ; d'ailleurs, nous avions déjà signalé l'analogie des deux dépôts, et aujourd'hui leur synchronisme est pour nous un fait acquis. 


\title{
II \\ DÉPOTS DU PUY-DE-DONE
}

\author{
DÉPOT DU CHAMBON
}

Le dépôt du Chambon est situé à 880 mètres d'altitude, près du bord Est du lac Chambon; il est formé par un mélange de roches d'un blanc pur ou d'un jaune pâle, de consistance ferme ou friable; le volume de la masse est à peine de quelques centaines de mètres cubes.

Dans sa Flore pliocène du Mout-Dore, l'abbé Boulay mentionne ce dépôt, mais il le croyait très probablement dépourvu de Diatomées, attendu que les échantillons reçus de notre très regretté et éminent ami, nous furent adressés sous le nom de tripoli du Chambon; parmi ces échantillons il s'en trouva, en effet, qui étaient exclusivement formés de silice amorphe à ćléments trìs fins, tandis que d'autres, tout ì fait analognes anx premiers par leur faciès lithologique, contenaient des Diatomées en assez grande abondance.

Ce dépôt renferme quelques empreintes de feuilles appartenant à la flore actuelle (Fagus, Salix, Almus, etc.), c'est donc un lambeau remanié, comme l'est son voisin, le beau dépôt de Varenne, dont la flore phanérogamique a été magistralement étudiée par l'abbé Bou. lay, et la flore diatomique plus modestement par nous dans le Mémoire de 1893. 
Les Diatomées observées sont :

Cocconeis lineata Grun. (Diat. d'Auv., p. 44).

- $\quad$ - var. euglyptaGr.(D.d'Auv.,p.46).

- Placentula Ehrb. (Diat. d'Auv., p. 44).

Cymbella gastroides Ktz. (Diat. d'Auv., p. 68).

Encyonemia lunula Grun. (Diat. d'Aur., p. 75).

- ventricosum Grun. (Diat. d’Auv., p. 73).

Epithema turgida Ktz. ( Diat. d'Auv., p. 124).

- - var. granulata (D.d'Auv., p.125).

- Zebra Ktz. (Diat. d'Auv., p. 127).

- - var. minor (Diat. d'Auc., p. 129).

Eunotia hungarica var. gracilior Pant.(Diat. Ung. III). - Diatomée nouvelle pour la flore française.

Fragilaria construens Grun. (Diat. d'Auv., p. 143). parasitica Grun. (Diat. d'Auv., p. 146).

Gomplonema intricatum Ktz. (Diat. d'Auv., p. 57).

Melosira crenulata Ktz. (Diat. d'Auv., p. 186).

- tenuis Grun. (Diat. d'Auv., p. 188).

Navicula Braunii var. moissacensis (D. foss. II, p. 63)

- cymbula Donk. (V. H. Syn.)

- major Ktz. (Diat. d’Auv., p. 82).

- radians F. Hér. et MI. Per. (D. foss. II, p. 90)

- mupestris Ktz. (Diat. d'Auv., p. 84).

Stauroneis gracilis IV. Sm. (Diat. d'Aur., p. 76).

Synedra Ulna Ehrb. (Diat. d'Auv., p. 137).

Tetracychus Lancea M. P. etF.H. ( D. d'Auv., p. 159).

- rhombus Ralfs (Diat. d'Auv., p. 160).

Les diatomistes remarqueront dans notre liste l'absence complète des Coscinodiscus, tandis que ces Dia- 
tomées saumâtres sinon marines, sont fréquentes et variées à Varenne; la pliysionomie de la florule du Chambon, dénote un dépôt de formation postérieure à celle du dépôt de Varemne, lequel nous semble bien appartenir au Miocène supérieur par sa florule diatomique, et au Pliocène par sa florule phanérogamique; l'abbé Boulay croyait aussi le dépôt du C'hambon plus récent que Varenne, ̀̀ cause de sa florule phanérogamique quaternaire, mais il ne lui a pas assigné de date fixe, ne connaissant pas la florule diatomique, laquelle est bien différente de celles des dépôts quaternaires des monts Dômes que nous arons étudiés avec le plus grand soin pour la publication du Mémoire de 1893.

D'après les caractères de ses deux florules diatomique et phanérogamique, le dépôt remanié de Chambon doit être daté du Pliocène, et son remaniement a dî s'effectuer, avec la fossilisation des feuilles qu'il contient, au début du quaternaire, à l'époque oì les épanchements laviques du Tartaret obstruèrent la vallée et formèrent ainsi le lac Chambon (lac de barrage). A propos du dépôt remanié de St-Saturnin, nous avons fait observer que sa florule phanérogamique est nettement quaternaire, tandis que la florule diatomique a tous les caractères d'une florule pliocène. Le Chambon nous fournit un second exemple de ce cas fort intéressant.

Quant au remaniement de Tareme, il dut se faire très probablement, au début du Pliocène, avec les émis. sions trachytiques et andésitiques des grands volcans des Monts Dores.

Parmi les autres dépôts du Puy-de-Dôme laissés à M. Lauby pour la publication prochaine de sa thèse inaugurale, celui du bois du Capucin, prìs du Mont- 
Dore, et des affleurements nouveaux du dépôt initial des Egravats, sont particulièrement intéressants, à cause des espèces nouvelles qu'ils nous ont livrées.

Le dépôt du Capucin, découvert par nous en 1904, et étudié avec soin par M. Iauby, ne contient pas de feuilles fossiles; il doit être un prolongement non remanié de celui des Egravats, dont il n'est séparé que par la vallée des Bains, dont le creusement est postérieur à la formation miocène du dépôt.

Les espèces ou variétés observées sont de 54, comprenant trois espèces nouvelles : 1 Cymbella et $2 \mathrm{Na}$ vicula.

Le dépôt des Egravats, étudié sommairement dans notre Mémoire de 1902, sur un échantillon unique et très paurre de la collection Bouillet, a été exploré en 1906 par M. Lauhy; le nombre des espèces ou variétés observées dans les matériaux nombreux et variés, provenant de plusieurs affleurements nouveaux est d'environ 80 , comprenant 6 espèces nouvelles et 7 variétés.

Ces formes inédites appartiennent aux genres:

Aclinanthes, Cymbella, Navicula, Diatoma, Epithemia et Fragilaria.

Le nombre des espèces ou variétés inédites décrites et figurées dans le présent Mémoire est de 55 , et en ajoutant les 16 formes observées par M. Lauby, et qui seront publiées prochainement, la flore diatomique du Massif Central se trouve augmentée de 71 espèces ou variétés nouvelles et très distinctes; le genre Anorthoneis est une bonne acquisition pour la flore diatomique du Cantal. 


\section{DÉPOTS DE LA HAU'TE-LOIRE}

C'est à M. le professeur Gallaud, Docteur ès-scieñces, que nous devons la totalité des matériaux étudiés provenant des dépôts inédits de Mardansou et d'Andreugeolet découverts par lui, ainsi que les échantillons du dépôt ligniteux d'Araules, déjà connu de M. Boule, mais dont la florule diatonique n'avait pas été encore étudiée.

Au cours de ses laborieuses recherches sur les dépôts fossilifères de la Haute-Loire et de l'A rdèche, M. Gallaud à recueilli des matériaux considérables et absolument inédits, concernaut la flore phanérogamique des dépôts nouveaux, et de plusieurs autres déjà connus, mais trop sommairement étudiés par de Saporta, Boule et abbé Boulay, tels que les beaux dépôts de Ceyssac, La Roche-Lambert, le Monastier, Araules, etc. (HauteLoire), et Gourgouras, Ranc, Charray, Pourchères, etc. (Ardèche). L'étude de ces matériaux du plus haut intérêt est déjà bien avancée, et M. Gallaud nous fera conuaitre prochainement le résultat de ses belles découvertes.

N'ayant pu visiter encore les dépôts de la HauteLoire, et encore moins ceux de l'Ardèche, nous n'arons pu les étudier que d'après les échantillons reçus de ros correspondants. 


\section{DÉPOT DE MARDANSOU}

Le dépôt de Mardansou est situé à 6 kilomètres de Langeac, au bas d'un ravin étroit et profond, à 1 kilomètre à l'E. de Saint-Arcon-d'Allier; son altitude est d'environ 530 mètres. Il affleure sur une longueur de 50 mètres, dans le lit même du petit ruisseau affluent de l'Allier, et présente une épaisseur visible de 2 mètres environ. Il est très probable qu'il repose sur les micaschistes qui forment le substratum général de la région, et qui se montrent à peu de distance en aval dans le ruisseau.

L'argile qui le forme renferme de nombreuses paillettes de mica très fines, mais aucun élément basaltique. Dans la partie la plus inférieure de la couche risible, l'argile est très fine, compacte, non feuilletée et d'un gris noirâtre. La stratification qui est sensiblement horizontale est peu marquće; elle devient plus apparente en hauteur, en même temps que l'argile devient plus grossière et un peu sableuse.

C'est dans la partie inférieure du dépôt que les Diatomées et les feuilles fossiles sont les plus abondantes; ces dernières sont toujours isolées et non groupées en lits comme le cas est fréquent dans les gisements de plantes fossiles.

Au-dessus des argiles, et les ravinant assez fortement, on trouve une couche d'alluvion de 3 mètres d'épaisseur environ, constituée par des cailloux bien arrondis, de la grosseur du poing, agglomérés par un ciment formé d'argile et de sable quartzeux. Les cailloux roulés 
sont en quartz pour la plupart, quelques uns en hasalte, mais beaucoup plus rares. Au-dessus enfin se trouve une coulée de basalte prismatique que le ruisseau franchit en cascade; le dépôt est donc nettement sous basaltique.

De cette disposition stratigraphique et de la nature pétrographique du dépôt fossilifère, on peut conclure qu'il s'est formé aux dépens du terrain primitif sousjacent, à une époque où les éruptions volcaniques de la chaîne du Velay n'avaient pas encore commencé, ou du moins ne s'étaient pas encore étendues dans cette région.

L'examen des échantillons nombreux et variés, reçus de M. Gallaud, nous a donné le résultat suivant :

Rhoicosphenia curvata Grun. (Diat. d'Auv., p. 51).

Cocconeis Bonnieri F. Hérib. (Diat. foss., II, p. 59).

-- intermedia M. P. et F. H. (D. d'Auv., p. 44).

- Placentula Ehrb. (Diat. d'Auv., p. 44).

Navicula ambigua Ehrb. (Diat. d'Auv., p. 108).

- appendiculata Ktz. var. major nov. Atteint $50 \mu$ de longueur.

- borealis Ktz. (Diat. d'Auv., p. 86).

Navicula borealis Ktz. var. linearis nou. (Pl. XIII, fig. 20). - Valve bacillaire à extrémités arrondies; raphé biarqué; stries robustes, au nombre de 4 en $10 \mu$, allant jusqu'au raphé d'un côté de la valve, beaucoup plus courtes et presque marginales de l'autre côté.

A. Schmidt (Atl. pl. 45, fig. 16), représente, sans lui donner de nom particulier, une forme analogue sans qu'elle soit identique. 
Navicula Brebissonii Ktz. (Diat. d'Auv., p. 88).

- gastrum Donk. (Diat. d'Auv., p. 102).

- halophila var. major F. Hér. (D. foss. II, p. 89).

Navicula halophila var. Eusebioi nov. (Pl. XIII, fig. 32). - Forme analogue à la variété major, mais moins grande; hords de la valve régulièrement convexes, extrémités coniques; pores terminaux légèrement récurvés. Longueur $80 \mu$, largeur $15 \mu$; stries au nombre de 16 en $10 \mu$.

Nous dédions ce joli Naricuia à II. le professeur Albert Eusébio, Docteur ès-sciences naturelles.

Navicula elliptica Ktz. (Diat. d' Auv., p. 104).

- Huei F. Hérib. (Diat. foss., II, p. 62); bien conforme à celui de Moissac.

- major Ktz. (Diat. d'Auv., p. 82).

- placentula Ehrb. (Diat. d'Aur., p. 102).

- radiosa Ktz. (Diat. d’ Auv., p. 99).

- Reinhardtï Grun. (Diat. d'Auv., p. 102).

- spleceroplora Kitz (Diat. d'Auv., p. 109).

- stauroptera Grun. (Diat. d'Auv., p. 92).

Navicula subacuta var. minor nov. (Pl. XIII, fig. 21. - Valve bacillaire, à extrémités coniques arrondies; aire axiale étroite, subitement élargie en losange autour du nodule médian; stries nettes, non distinctement granulées, au nombre de 10 en $10 \mu$, légèrement radiantes au milien de la valve, convergentes aux extrémités. Longueur $70 \mu$, largeur $14 \mu$.

Differe du Navicula subacuta Ehrb. var. (A. Sch. Atl., pl. 43, fig. 31), par sa taille plus petite, par ses stries plus serrées, et par son aréa centrale plus grande. Naricula Termes (Diat. d'Auv., p. 97).

- transversa A. Sch. (Diat. foss., I, p. 14).

- rentricosa var. cuncate (Diat. foss., II, p. 59). 
Navicula viridis Ktz. (Diat. d'Auv, p. 84).

- - var. commutata (Diat. d'Auv., 1. 84).

Navicula mardansouensis sp.nov. (Pl. XIII, fig. 31). - Valve bacillaire, à extrémités longuement coniques et arrondies; raphé fin; aire axiale nulle, la centrale dilatée presque jusqu'aux bords de la valve; stries non distinctement granulées, les médianes très courtes, les voisines un peu plus longues, les suivantes allant jusqu'au nodule central, oì elles limitent une aréa centrale subquadrangulaire, très divergentes au milieu de la valve, convergentes aux extrémités. Longueur de la valve $60 \mu$, largeur $61 / 2 \mu$; stries au nombre de 10 en $10 \mu$ aux bords de la valve, les médianes plus écartées.

Espèce analogue au Naricula appendiculata Ktz., mais s'en distingue nettement par son aréa centrale non stauronéiforme, et par la forme de ses nodules terminaux.

Stauroneis acuta W. Sm. (Diat. d'Aur., p. 78).

- mesopachya Ehrb. (Diat. d’Auv., p. 79).

- Plecenicenteron Ehrb. (Diat. d'Aux., p.75)

I'an-Heurckia rhomboides var. amplipleuroides Grun.

(Diat. foss. II, p. 104).

Amphora ovalis Ktz. (Diat. d'Auv., p. 62),

Pleurosigma acuminatum Gr. (Diat. d'Aluv., p. 122).

Cymbella affinis Ktz. (Diat. d'Alu., p. 66).

- aspera Ehrb. (Diat. d'Auv., p. 69).

- austriaca var. fossilis (Diat. foss. II, p. 104)

- cistula fa curta (A. Sch., Atl., pl.10, fig. 24)

- lanceo'ata Ehrb. (Diat. d'Aun., p. 68).

- Laubyji F. Hérib. ( Diat. d'Auc., p. 18).

Cymbella Malinvaudi $s \mu^{\prime}$, nov. (Pl. XIII, fig. 4). - Face dorsale fortement convexe au milien, 
face ventrale droite, extrêmités coniques, légèrement produites dans une direction parallèle au bord ventral et largement arrondies; raphé fin, constitué par deux lignes droites formant un angle au nodule central, et légèrement récurvées vers le bord dorsal aux extrêmités; pores centraux petits, les terminaux faiblement développés; aire hyaline axiale étroite, peu dilatée autour du nodule médian; stries fortes, distinctement et finement divisées en travers, fortement radiantes à la partie dorsale, faiblement radiantes à la partie ventrale, où elles sont même légèrement convergentes aux extrêmités, au nombre de 6 en $10 \mu$ au milieu de la partie dorsale et de 10 en $10 \mu$ aux extrêmités, 9 en $10 \mu$ au milieu de la partie ventrale et très légèrement plus serrées aux extrêmités. Longueur de la valve $30 \mu$, largeur $23 \mu$.

Se distingue du Cymbella Balatonis Grun. (A. Sch. Atl., pl. 10, fig. 19), par sa partie ventrale plus étroite, plus finement striée, à bords plus rectilignes, et par ses nodules terminaux moins développés.

Cette belle espèce, très distincte, est dédiée à notre éminent ami, M. Ernest Malinvaud, ancien Président de la Société botanique de France.

Cymbella ovuin sp. nov. (Pl. XIII, fig. 5). Petite espèce de forme largement elliptique, à extrêmités très étroites et fortement rostrées; raphé fin et peu visible, légèrement arqué, divisant la valve en deux parties sensiblement égales; aire axiale nulle, la centrale très petite; stries fortement rayonnantes, au nombre de $9 \frac{1}{2}$ en $10 \mu$ au milieu de la partie ventrale, un peu plus serrées aux extrémités de la valve. Longueur du frustule $30^{1} / 2 \mu$; longueur du corps sans les extrémités $251 / 2 \mu$, largeur $13^{1 / 2} \mu$.

A. Schmidt (Atl..pl. 9, fig. 53) représente une forme 
analogue et la rattache an Cymbella cuspidata Ktz., mais sa forme est plus grande, les extrémités sont beaucoup moins rostrées et plus larges; les stries, au nombre de 7 en $10 \mu$, sont beaucoup plus écartées.

Cymbella Gallaudi sp. nov. (Pl. XIII, fig. 6). - Grande et belle forme; face dorsale fortement et régulièrement arquée, face ventrale faiblement concave et notablement gibbeuse au milieu; raphé régulièrement arqué, à nodules terminaux petits; aréa axiale nulle, la centrale très petite à la partie ventrale; stries fortes, formées de granules petite et rapprochés, au nombre de 9 en $10 \mu$ au milieu de la partie dorsale, de 10 en $10 \mu$ au milieu de la partie ventrale, un peu plus serrées vers les extrémités. Longueur 120 à $130 \mu$, largeur 22 à $25 \mu$.

Diffère du Cymbella lanceolata par sa forme plus courbée et plus large, et surtout par ses stries plus serrées.

Cette Diatomée, très distincte, est dédiée à M. le professeur Gallaud.

Encyonemu prostratum Ralfs (Diut. d'Auv., p. 72). ventricosum Ktz. (Diat. d'Auv., p. 73).

Fragilaria virescens Ralfs (Diat. d'Auv., p. 147).

Gomphonema geminatum Ag. (Diat. d'Aur., p. 52). - Cette espèce, exclusivement alpine, atteint jusqu'ì $120 \mu$ de longueur; les formes observées dans ce dépôt, tout en étant bien typiques, ont une longueur de $150 \mu$.

Gomphonema subcluxatum (ir. (Diat.d'Alur., 1.55). turgídum Ehrb. (Microgéol.)

Gomphonema marginatum sp.nor. (Pl. XIII, fig. 27). - - I'etit et étroit, de forme biconique, côtes de la valve légèrement concaves, extrémités largement ar- 
rondies ; raphé fin, à pores centraux et stigmate unilatéral bien visibles; aréa axiale très large, Jancéolée, non particulièrement élargie autour du nodule médian; stries très nettes, non distinctement granulées, très courtes, excepté à l'extrémité de la partie supérieure, presque marginales au milieu de la valve, où les médianes ne sont, ni sensiblement plus courtes, ni plus écartées que les autres, s'arrêtant assez loin de l'extrémité inférieure, où il existe une forte fissure. Longueur $37 \mu$, largeur $6 \frac{1}{2} \mu$; stries au nombre de 12 en $10 \mu$.

Se distingue d'une petite forme du Gomphonema brevistriata du dépôt de Neussargues, non seulement par sa taille et ses stries plus serrées, mais encore par son aréa, qui est relativement plus large et non particulièrement arrondie autour du nodule médian. - Petite espèce très distincte.

Eunotia gracilis Rab. var. robusta nor. (Pl. XIV, fig. 1). - Valve grande et robuste, légèrement arquée, à bords parallèles, le bord dorsal, sensiblement infléchi près de l'extrémité, rend celle-ci un peu capitée, mais non récurvée; pores terminaux grands et bien visibles; stries robustes, obscurément divisées en travers, au nombre de 8 en $10 \mu$ à la partie médiane, et de $9^{1 / 2}$ en $10 \mu$ à la partie étranglée qui précède l'extrémité. Longueur 140 ì $150 \mu$; longueur du fragment observé $95 \mu$; largeur $9 \mu$.

Eunotia monodon var. diodon Ehr. (Diat. d' Auv.,p. 133).

- incisa Greg. (Diat. d'Auv., p. 133).

- - var. obtusiuscula(V.H.Syn.pl. 34, fig. $\left.35^{b}\right)$.

- prevupta Ehrb. var. bidens (Syn. pl. 34, fig. 22).

- polyglyphis Grun. (Diat. d'Auv., p. 134).

- - var. pentaglyphis Grun.

- - rar. hexaglyphis nov. 
Eunotia polyglyphis var. octoglyphis nor.

- - var. decaglyphis nor.

Synedra rumpens var. fragilarioides (D. d'Aur., p. 92).

- Ulna Ehrb. var. cequalis Grun.

- - var. undulata nov. - Un fragment très fortement ondulé.

Epithemia Argus K'tz. (Diat. d'Auv., p. 127).

- var. amplicepliala (D.d'Auv., p.127).

- gibba Ktz. (Diat. d'Auv., p. 126).

- - var. ventricosa Gr. (D. d'Auv., p.126).

- IIyndmannii W. Sm. (Diat. d'Aun., p. 125).

- _ - var. perlonga (Diat. foss., II, p. 28).

- Sorex Ktz. (Diat. d'Auv., p. 126).

- turgida Ktz. (Diat. d'Auv., p. 124).

- Westermannii Ktz. (Diat. d'Auv., p. 126).

- Zebra Ktz. Diat. d'Auv., p. 127).

- _ var. minor F ${ }^{\mathrm{re}}$ H. (Diat. d'Auv., p. 129).

- _ - var. preboscidea (Diat. d'Auv., p. 128).

IIantzschia amphioxys Grun. (Diat. d'Auv., p. 162).

- $\quad$ - var.major Gr. (D. d'Auv., p. 162).

Nitzschia granulata Grun. (V. H. Syn.).

Cymatopleura elliptica IV. Sm. (Diat. d'Aur., p. 160).

Opephora Martyi F. Hérib. (Diat. foss. I, p. 43).

Campylodiscus costatus II. Sm. (Diat. d'Aur., p. 182).

Diatoma Boulei sp. nov. - Analogue ou Diatoma hyemale, dont il se distingue par les côtes qui sont placées au fond de petites dépressions, de sorte que le frustule présente, en vue valvaire et en vue connective, un aspect ondulé; valve lancéolée avec des extrémités atténuées: pseudo-raphé assez apparent; côtes 4 à 6 en $10 \mu$, s'arrêtant au pseudo-raphé, et souvent non opposées des deux côtés de la valve; stries peu visibles, au nombre de 20 en $10 \mu$. Longueur 40 à $45 \mu$. - Se trouve aussi dans le dépôt initial des Egravats (Mont-Dore). 
Diatoma vulgare Bory . (Diat. d'Auv., p. 151).

'Tabellaria fenestrata Ktz. (Diat. d'Auv., p. 15t). - ventricosa Ktz. (Microgéol.).

Tetracychus stella (Ehrb.) F. H. (Diat. foss. I, p. 17). - emarginatus W. Sm. (Diat. d'Auv., p. 158).

Tetracyclus emarginatus var. integriloba nor. (Pl. XIV, fig. 22). - Se distingue du type de IV. Smith par ses dimensions plus grandes. Longueur 45 i $50 \mu$, au lieu de $30 \mu$, par la dilation centrale, dont les deux lobes sont terminés par des côtés droits, parallèles au raphé, au lieu d'être infléchis au milieu, comme ceux du type, et par les extrémités arrondies, au lieu d'être acuminées.

Tetracyclus major sp. nor. (Pl. XIV, fig. 23). - Grand et robuste; de forme analogue à celle du Tetracyclus emarginatus; côtes nombreuses, relativement faibles, et dont beaucoup sont incompletes; stries transversales très fines, indistinctement granulées, non visiblement interrompues par un pseudoraphé. - Longueur 50 ̀̀ $65 \mu$, largeur 35 à $40 \mu, 2$ à 3 côtes en $10 \mu$. - W. Smith, donne $30 \mu$ de longueur à son Tetracyclus emarginatus type.

Tetracyclus major var. acuminata nor. (Pl. XIV, fig.24). - Diffère du type par les extrémités acuminées au lieu d'être arrondies. - Longueur 65 à $70 \mu$.

Tout en différant du Tetracyclus emarginatus IV. Sm., par leur grande taille et par plusieurs détails, ces trois dernières formes ont une grande analogie avec l'espèce de W. Smith, dont elles pourraient bien être des mégafrustules; cependant elles ne sont pas plus rares dans le dépôt que le Tetracylus emarginatus $\mathrm{W}$. 
Sm., et leurs caractères différentiels étant reconnus bien constants, il nous a paru utile de les distinguer.

Melosira loevis Grun. (Diat. d'Auv., p. 187).

- arenaria More (Diat. d'Aur., p. 186).

Meridion constrictum Ralfs (Diat. d'Auv., p. 153).

Cyclotella Temperei F. Hérib. (Diat. d'Aur., p. 231). Cette espèce, la plus commune du dépôt, est très variable comme grandeur; il n'est pas rare d'observer des exemplaires dont le diamètre atteint près de $50 \mu$.

Stephanodiscus Astrcea Ktz. (Diat. d'Auv., p. 193). Très fréquent, mais peu visible.

Par la grande abondance du Cyclotella Temperei et du Stephanodiscus Astrcea, espèces caractéristiques des dépôts tertiaires de La Bourboule et de Saint-Saturnin (Puy-de-Dôme), Mardansou à bien quelque analogie avec ces deux dépôts, mais par l'ensemble de sa florule il est bien distinct; il pourrait être comparé aussi au dépôt d'Andelat (Cantal); les deux dépôts ont en effet plusieurs espèces communes, sans qu'il soit possible cependant de les identifier.

En attendant la publicatipn prochaine de la florule phanérogamique de Mardansou, par M. Galland, laquelle nous permettra de fixer la date du remaniement de ce beau dépôt, nous devons nous borner à constater que sa florule diatomique est tout à tait différente de celles des autres dépôts connus de la Haute-Loire.

La physionomie de cette belle florule est celle d'une formation nettement lacustre; le dépôt initial a dî s'ếfectuer dans un lac d'eau douce situé à une altitude bien supérieure à 530 mètres, à cause des espèces froides observées dans ce lambeau remanié. Le dépôt n'a pu se former dans un cratère-lac trachytique dont les eaux plus ou moins minéralisées auraient permis aux Coscinodiscus, aux Stictodiscus, aux G'oniothecium, aux 
Terpsinoe, etc., Diatomées marines, ou tout au moins saumâtres, de vivre et de se multiplier; or, ces espèces fout ici absolument défaut, tandis qu'elles sont assez fréquentes dans les autres dépôts tertiaires du Massif Central, en particulier dans celui de Ceyssac.

En résumé, Mardansou est pour nous un lambeau remanié, provenant d'un dépôt lacustre appartenant à un système de formation différent de Ceyssac, Vals, le IIonastier, Gourgouras, elc.

\section{DÉPOT D`ANDREUGEOLET}

Le dépôt d'Andreugeolet est situé à quelques centaines de mètres au Nord du hanreau d'Andreugeolet, canton de Saugues.

Ce dépôt est très riche en grandes et belles formes, et à valves peu fragmentées; les espèces observées appartiennent surtout à la catégorie des Diatomées littorales ou épiphytes; les espèces pélagiques ou vaseuses sont beaucoup plus rares. Les Epithemia et les Nivicula sont largement représentés comme espèces, et celles-ci très abondantes comme individus.

Nous n'avons pu voir encore le dépôt en place; d'après M. Gallaud, il affleure à l'altitude de $930 \mathrm{~m}$. sur la ligne de contact de la petite trainée basaltique qui borde la vallée de la Seuge, et du granite porphyroïde qui constitue le haut plateau entre Saugues et Monistrol-d'Allier. La couche à Diatomées a une épaisseur d'environ 2 mètres; la stratification, peu marquée, est 
sensiblement horizontale; les échantillons reenus sont de couleur blanche, friables et très légers; la masse est très homogène et sillonnée en tous sens par les racines des plantes qui se développent ì la surface du sol. Au dessus et au dessous de la couche à Diatomées se montrent des sables granitiques très variables d'allure, tantôt très fins, tantôt assez grossiers; ceux de la base reposent sur le granite; ceux du sommet passent sous les brèches hasaltiques qui se montrent ì quelques mètres de distance, sans qu'il soit possible d'être très affirmatif à cause de la végétation qui masque les points de contact, et à cause aussi de l'allure très irrégulière des brèches basaltiques.

II. Gallaud n'a pas trouvé d'empreintes de feuilles dans la couche à Diatomées, ni dans les sables qui l'encaissent, mais il est possible que des recherches ultérieures permettent d'en découvrir, au moins dans la masse diatomilère.

Voici la liste établie d'après l'examen des échantillons reçus de M. Gallaud:

Aclenantles linceolata Bréb. (Diat. d'Aux., 1'. 49).

Amphora enoculata $s p$. not. (Pl. XlII, fig. 3). -- Valve à face dorsale fortement et régulièrement courbée, face ventrale droite, excepté aux extrémités, où elle est faiblement proéminente; extrénités largement arrondies; raphé droit, peu apparent, placé tout à fait sur l'arête de la valve; stries rayomnantes, au nombre de 10 en $10 \mu$ au milieu de la valve, un peu plus serrées vers les extrémités, coupées, vers le nilieu de leur longueur, par un pli peu prononcé, sur lequel elles sont brisées, les deux parties formant un angle très ouvert, visible surtout vers les extrémités. - Longueur de la valve 75 ì $80 \mu$. 
Ressemble ì l'Amphora oculus A. Schm. (Atl., pl. 27, fig. 52), mais sans l'affaiblissement des stries médianes formant l'oculus; ressemble aussi à la fig. 56 de la même plauche non dénommée par Schmidt et provenant de Samoa.

Amploora oralis Ktz. (Diat. d'Aur., p. 62).

- Pediculus Grun. (Diat. d'Auv., p. 63).

Coccone is lineata Crun. (Diat. d'Aur., p. 44).

- Placentula Ehrb. (Diat. d'Aux., p. 4t).

Cyclotella comtu var. quadrinotutu ( D. foss. II, p. 108).

- Temperei F. Héril. (Diat. d'Aur.p. 231).

Cymatopleura elliptice var, rhomboides (V. H. Syn.) Solea IV. Sm. (Diut. d'Aur., p. 161).

Cymbella gastroides var. prolongata nor. (Pl. XIII, fig. 12). -- Se distingue du type par ses extrémités tronquées et prolongées, l'extrémité du bord dorsal étant légèrement concave au lieu d'être convexe. Longueur $180 \mu$; stries au nombre de 6 en $10 \mu$ au milieu du bord dorsal, 7 en $10 \mu$ au milieu du bord ventral, 10 en $10 \mu$ aux extrémités.

C'ymbella gastroides Ktz. (Diat. d'Aux., p. 68).

- cistula var. fusidium (Diat. d'Auv., p.71).

- helecticu Ktz. (Dint. d'Auv., p. 72).

- lanceolata Ehrb. (Diat. d'Auv., p. 68).

- leptoseras Ehrb. (Diat. d'Aur., p. 66).

- maculata Ktz. (Diat. d'Aur., p. 71).

Cymbella gallica sp. nox. (I'l. XIII, fig. 7). - Valve à face dorsale faillement et régulièrement convexe, face ventrale très légèrement convexe, extrémités obtuses arrondies; raphé bifide et légèrement biarqué, à nodules terminaux eu flamme, à pores terminaux très petits et éloignés des extrémités; aire axiale 
très étroite, surtout du côté ventral, légèrement arrondie autour du nodule médian; stries très fortes, finement mais très distinctement divisées en travers, radiantes, allant jusqu'aux extrémités de la valve, où elles entou. rent les nodules terminaux, au nombre de 7 en $10 \mu$ au milieu de la partie dorsale, de $8 \frac{1}{2}$ en $10 \mu$ au milieu de la partie ventrale, légèrement plus serrées aux extrémités. Longueur de la valve 98 à $100 \mu$.

A quelque ressemblance avec le Cymbella helvetica Ktz., mais il s'en distingue nettement par sa forme moins élancée, convexe des deux côtés, par ses extrémités non récurvées, et par son raphé bifide.

Encyonema Auerswaldï Rab. (Diat. d'Aur., p. 73 ). -. ccespitosum Ktz. (Diat. d'Aur., p. 73).

- turgilum Greg. (Diıt. d'Auv., p. 73).

Epithemia gibba Ehrb. (Dirt. d'Auv., p. 126).

- var, rentricosa (Diat. d'Auv., p. 126).

- HynulmumnïW. Sm. (Diat. d'Auv.,p.125)

- $\quad$ - var.perlonga (D.foss. II, p. 28)

- Sorex Kitz. (Diat. d'Aus., p. 126).

- turgida Kitz. (Diat. d'Auv., p. 124).

- $\quad$ - var.gramulate (D.d'Aur., p. 125)

- - var. Tertagus (D. d'Aue, p.125)

- Zebra Ktz. (Diat. d'Auv., p. 128).

- _ - var.proboscidea (D).d'Auv.,p.128)

- - var. minor (Diat. d'Aur.,p.129).

Eunotia pectinalis var. stricta (Diat.d'Aur., p.132).

Fragilaria elliptica Schum. (Diat. d'Aur., p. 145).

- parasitica Grun. (Diat. d’_luc., p. 146).

Gomphonema acuminatum Ehrb. (Diat.d'Aux.,p. 61)

- - var.laticeps (D.d'Auv.,p.5t).

- - var.pusilla (I).d'Aur.,p.5t).

-_ constrictum Ehrb. (Diat. d'Auv., p. 52). 
Melosira Mauryana var. granulosa nor. (Pl.XIV, fig. 30). - Diffère du type de La Garde en ce que, sur la vue valvaire, on apercoit non seulement une ligne de granules bordant la paroi, et placée sur la face interne de l'anneau valvaire, mais encore une deuxième ligne de granules intérieure à la première et placée sous la valve même.

Naricula arenaria Donk. (V. H. Syn.).

- borealis Ehrb. (Diat. d'Auv., p. 86).

- cardinalis Ktz. (Diat. d'Auv., p. 85).

- Chaberti F. Hérib. (Diat. foss. II, p. 60).

- commutata Grun.

- cuspidata Kiz. (Diat. d'Auv., p. 105).

- elliptica Kitz. (Diat. d'Aur., p. 104).

- Esox Ehrb. var. recta $\mathrm{F}^{\mathrm{re}}$ H. (D. foss., II, p. 11).

- yentilis Donk. (Diat. d'Aur., p. 81).

- haloplita Grun. (Diat. foss., II, p. 103).

-- $\quad$-- var. major (Diat. foss., II, p. 89).

- Meribaudi M. Per. (Diat. d'Aux., p. 112).

- hemiptera Ktz. (Diat. d'Aur., 1. 84).

- limsa Kitz. (Diat. d'Aur., p. 111).

- major Ktz. (Liat. d’Aur., p. 82).

- nobilis Ehrb. (Diat. d'Aue, p. 80).

- Placentula Ehrb. var. minor nor. - Se distingue du tỵpe par sa taille très réduite; long. 30 à $35 \mu$. Naricula radiosa Ktz. (Diat. d’Auv., p. 99).

- - var. acutu Grun. (Diat. d'Aur., p. 100).

- - var. maxima nov. - Atteint la longueur de $120 \mu$, avec 8 stries en $10 \mu$ ì la partie médiane de la valve.

Nacicula Reinharditi Grun. (Diut. d'Aur., p. 102).

- rupestris Ktz. (Dirt. d'Aur., p. 84).

- transversa A. Sch. (Diat. foss., I, p. 14).

- rentricosa Donk. (Diat. d’Auv., p. 113). 
Naricula viridis Ktz. (Diut. d'Aur., p. 83).

Nitzschia sigmouidea W. Sm. (Dirt. d'Alur., p. 167).

Pleurosigma KutzingüGrun. (Dist. d’Aur., p. 123).

Stiuroneis Buileyi Ehrb. (Diut. foss., II, p. 67).

- grucilis W. Sm. (Dicit. d'Aw., p. 76).

- Phicenicenteron Ehrb. (Diat. d'Auv., p. 75).

- pteroides Ehrb. (Microgéol.).

Surivella robusta Ehrb. (Diat. d'Auv., p. 180).

- - var. A. Sch. (Atl., pl. XXII, fig. 4).

- splendida Ehrb. (Dict. d'Aur., p. 175).

Synedra Clna Ehrb. (Diat. d'Aur., p. 137).

Tetrucyclus emarginutus IV. Sm. (Diut.d'Auv., p. 158).

En lisant cette liste, on voit que la florule d'Andreugeolet, comme celle de Mardansou, ne comprend que des espèces lacustres; il en résulte que ce dépôt a dû se former dans un lac d'eau donce; l'ensemble de la florule dénote aussi une formation postérieure à celle des dépôts tertiaires du Massif Central, dans lesquels on troure des espèces saumâtres, mais nous ne sommes pas encore suffisamment documenté pour pouvoir lui attribuer une date fixe.

II. Gallaud n'a pu examiner Andreugeolet que très sommairement; le peu de temps dont il disposait ne lui a même pas permis de vérifier si le dépôt contient ou non des empreintes de feuilles, par conséquent nous ne savons pas si nous avons à étudier un dépôt en place ou un lambeau remanié, et c'est là un point qu'il serait important d'élucider.

En attendant le résultat de recherches ultérieures, Andreugeolet nous semble très analogue à Mardansou, et appartenir comme celui-ci à un même système de formation diatomique différent de celui des autres dépôts de la Haute-Loire et de l'Ardèche. 


\section{DÉPOT D'ARAULES}

Le dépôt d'Araules, situé au S.-E. d'Yssingeaux, signalé par notre savant compatriote, M. le professeur Boule, paraît assez étendu, si on en juge par les exploitations de lignite auxquelles il a donné lieu, mais nulle part il n'affleure à la surface, ou du moins il est partout masqué par les éboulis ou la végétation. On ne peut se faire une idée des roches qui le composent qu'en examinant les déblais résultant des anciennes galeries d'exploitation abandonnées depuis longtemps. On y trouve des sables, des argiles vertes, des rognons de silice concrétionnée renfermant des débris de feuilles et des fruits; des schistes blancs et bruns diatomifères, ì texture très fine, et des schistes ligniteux charbonneux avec coquilles de lymnées écrasées.

Trois échantillons de ces schistes nous ont été envoyés par M. Gallaud; ils sont très différents, tant pour la couleur que pour leur florule respective; ils proviennent sans nul doute de couches absolument distinctes ; il serait très intéressant de connaître leur ordre de succession verticale et de prélever des échantillons à des hauteurs intermédiaires.

Les préparations examinées nous ont donné le résultat suivant :

Achnanthes lanceolata Bréb. (Diat. d'Aux., p. 49).

Amphora araulensis sp. noi. (PI. XIII, fig. 2). - Valve cymbiforme; face dorsale fortement courbée, 
le milieu légèrement aplati, face ventrale un peu concave, extrémités fortes et capitées ; raphé lien visible, arqué suivant le bord rentral, à pores du nodule central ronds et rapprochés; stries fines, rayonnantes jusqu'aux extrémités de la valve, assez bien marquées, surtout ceiles de la partie centrale de la valve, qui sont beaucoup plus fortes et plus écartées que les autres, au nombre de 18 en $10 \mu$ au milieu de la valve, beaucoup plus serrées aux extrémités. Longueur du frustule $35 \mu$, largeur $7 \mu$.

Amphora protracta Pant. var. gallica nor. (Pl. XIII, fig. 1). - Valve cymbiforme, à face dorsale fortement et régulièrement courbée, face ventrale concave, extrémités nettement capitées et récurvées du côté du bord dorsal ; raphé fin mais bien visible, constitué par deux lignes à peu près droites, formant un angle ouvert au nodule central, lequel est assez éloigné du bord ventral, pores droits, écartés l'un de l'autre ; stries fines mais très nettes, formées de points allongés et bien distincts, régulièrement rayonnantes jusqu'aux extrémités, où elles sont un peu plus serrées qu'au milieu de la valve; zone connective complexe, formée de nombreux plis lisses. Longueur de la valve 40 ¿ $45 \mu$, largeur $8 \mu$; stries 18 en $10 \mu$.

Se distingue du type (Iant. Ung. II, pl. 1, fig. 5), par sa longueur moindre, ses extrémités plus capitées et son raphé plus angulaire.

Navicula dubravicensis Pant. var. gallica nor. (Pl. XIII, fig. 22). - Valve longuement lancéolée, ¿̀ extrémités légèrement produites et finement arrondies; aire axiale lancéolée, assez large, mais non particulièrement arrondie autour dı nodule central; stries 
très radiantes au centre de la valve, où les médianes sont alternativement longues et courtes, se redressant progressivement, celles des extrémités sont convergentes et plus serrées qu'au milieu de la valve. Longueur $60 \mu$, largetir $11 \mu, 9$ stries en $10 \mu$ au milieu de la valve, 15 en $10 \mu$ aux extrémités.

Diffère du Naricula dubravicensis Pant. (Pant. Lng. III, pl. 19, fig. 281), du dépôt de Dubravica (Hongrie), par sa forme plus élancée, par ses extrémités beaucoup plus fines et produites, et par ses stries terminales convergentes et plus serrées.

Navicula aquitanice F. Hérib. (Diat. d'Aux., p. 81).-La découverte de cette espèce du dépôt de Mur, dans celui d'Araules est très remarquable et bien inattendue.

Navicula bolemica Ehrb. (Microgéol.).

- sculpta Ehrb. (Diat. foss. I, p. 41).

- mpestris Kutz. (Diat. d'Awv., p. 84).

Nitzsclia sigmoidea W. Sm. (Diat. d'Aur., p. 167).

Cymbella miocenica sp. nov. (Pl. XIII, fiig. 13). - Valve à face dorsale fortement et régulièrement courbe, face ventrale droite et légèrement gibheuse; extrémités coniques et largement arrondies; raphé lien visible, pores centraux droits et un peu éloignés l'un de l'autre, les terminaux ronds, assez éloignés des extrémités, placés au centre du cercle formé par l'extrémité de la valve et prolongés en flamme vers le bord dorsal ; aire axiale lancéolée, large, non sensiblement arrondie autour du no lule médian; stries nettes et fortes, finement divisées en travers, régulièrement divergentes jusqu'aux extrémités, an nombre de 9 en $10 \mu$ au milieu de la face ventrale, plus serrées aux extrémités. Longueur $40 \mu$, largeur $10 \mu$.

Ad. Schmidt (4tl., pl. 71, fig. 23) figure, sans la 
nommer, une forme du dépôt d'Ordie, analogue ì notre C'ymbella d'Araules, mais beaucoup plus petite et dont les détails de la striation sont différents.

Cymbella cymbiformis Ehrb. (Diat. d'Aux., p. 69). leptoceras Ktz. (Diat. d'Auv., p. 66).

Gomphonema Gallaudi sp. nor. (Pl. XIII, fig. 19). - Valve biconique, à extrémités légèrement atténuées et largement arrondies; aire axiale notable, très peu élargie autour du nodule médian du côté du stigmate, presque stauronéiforme de l'autre côté, par suite du raccourcissement de la strie médiane et du grand écartement des deux stries voisines; stigmate formé de trois points très rapprochés les uns des autres, placés très près du raphé et fortement marqués; stries radiantes jusqu'aux extrémités de la valve, fines, non distinctement granulées et différemment disposées des deux côtés de la valve; du côté du stigmate elles sont plus serrées au milieu de la valve $(8 \%$ en $10 \mu)$ qu'aux extrémités ( 7 en $10 \mu)$, du côté opposé elles sont plus écartées au milieu ( 6 en $10 \mu$ ) qu'aux extrémités 7 en $10 \mu)$. Longueur de la valve $60 \mu$, largeur $11 \mu$.

II. Fricke représente (A. Sch. Atl. pl. 235, fig. 1) une forme analogue, mais elle ne présente pas la même dissymétrie des stries, de plus les stries terminales sont beaucoup plus serrées (12 à 13 en $10 \mu$ ); peut-être à cause de ses côtes concares il la désigne suus le nom de Complonema intricatum var. fossile. Notre Diatomée, par sa forme extérieure, son nombre de stries et leur disposition ne peut être rapportée au Gomphonemu intricatum. - Cette espèce, très distincte, est dédiće à M. le professeur Gallaul.

Gomplionema intricutum var.dichotoma 'D.1'A., p.219) + insigne Greg. Diut. l' lux., p. 59). 
Fragilaria endocystifera $s p$. nor. (Pl. XIV, fig. 10 et 11). - Diatomée très curieuse, paraissant formée d'un frustule de Fragilaria très délicat renfermant un endocyst lisse très robuste; elle se présente presque toujours par sa face connective montrant l'endocyst, une seule valve du frustule, une partie de l'anneau connectif, et, quelquefois, adhérente à la valve isolée du frustule voisin.

Les vues valvaires sont très rares et ordinairement incomplètes,

Valves (frustule et endocyst) bacillaires ou elliptiques très allongées; l'endocyst est lisse, mais la valve est striée transversalement sans pseudo-stauros risible; la face connective du frustule est bacillaire, tronquée, striée sur les bords, celle de l'endocyst est bacillaire, atténuée aux extrémités, absolument tronquée et ayant même les angles épineux vers les côtés. Longueur 30 à $150 \mu$, largeur de la valve 5 à $10 \mu$, épaisseur du frustule 8 à $15 \mu$.

Fragilaria araulensis sp. nox. (Pl. XIV,fig. 6 , $7,8)$. - Très variable comme forme et comme dimensions; les petits exemplaires, souvent les plus larges, sont elliptiques allongés (fig. 7 ), les grands sont bacillaires allongés, à extrémités ordinairement atténuées et arrondies (fig. 8); pseudo-raphé nul, représenté seulement par les extrémités des stries qui ne se correspondent pas toujours des deux côtés de la valve; stries fines, mais bien marquées, délicatement granulées, arquées vers les extrémités, assez régulièrement espacées; face connective quadrangulaire (fig. 6), à angles un peu arrondis et présentant un prolongement des stries assez long, égal sur la plus grande partie de la valve et diminuant rers les extrémités. Longueur de 30 à $80 \mu$, largeur 8 à $13 \mu$; stries au nombre de 9 en $10 \mu$. 
Fragilaria breristriata Grun. (Diat. d'Aur., p. 146). - construens Grun. (Diat. d'Aux., p. 143). - elliptica Schum. (Diat. d'Aur., p. 145).

Eunotia gracilis Rab. (Diat. d'Aur., p. 131).

- lunaris Grun. (Diat. d'Auv., p. 135).

Encyonema ventricosum Ktz. (Diat d'Auv., p. 73).

Melosira crenulata Ktz. var. armata Gr. (V. H. Synn.) - distans Ehrb. var. scalaris (D. foss. II, p.94). - undulata Ktz. (Diat. foss. I, p. 22).

Les échantillons étudiés contiennent aussi une grande quantité de petits fragments de valves, mais absolument indéterminables; cette fragmentation des frustules est due très probablement à la pression énorme produite par l'accumulation des roches sous lesquelles est située aujourd'hui la masse diatomifère, et aussi à la trituration produite par le remaniement du dépôt.

II. Boule assimile le lambeau remanié d'Araules aux alluvions sous-basaltiques des Coirons, c'est-ì-dire au Miocène supérieur.

N'ayant pu voir encore le dépôt en place, nous ne pouvons mieux faire que d'adopter l'opinion du savant professeur du Muséum.

\section{DÉPOT DE GOURGOURAS}

Ce dépôt nous était déjà connu par l'examen d'un échantillon reçu de notre regretté et savant ami, l'abbé Boulay, et dans lequel nous avions observé une douzaine d'espèces, publiées dans le Mlémoire de 1903, p, 121. 
C'est à M. le professeur Gallaud que nous devons les échantillons étudiés ici et qui nous ont livré les formes suivantes :

Coscinodiscus Boulei (Diat. foss. II, p. 101).

Encyonema ventricosum Ktz. (Diat, d'Auv., p. 73).

Epithemia gibberula Ehrb. (Liat. d’Auv., p. 129).

Eunotic polyglyphis Grun. (Diat.d'Auv., p. 134).

Fragilaria Zeilleri F. Hérib. (Diat. foss. II, p. 26).

Melosira granulata Ehrb. (Diat. d’Auv., p. 186).

- spiralis Ktz. (Diat. foss. I, p. 17).

- tenuis Grun. (Diat. d'Auv., p. 188).

Navicula gigas Ehrb. Diat. d'A A x., p. 81).

- major Ktz. Diat.d'Aur., p, 82).

Tetracyclus Peragalli F. Ilérib. Diat. foss. II, p. 31). - - var. eximia F.H. (Diat. foss. II, p. 31).

En comparant cette florule avec celle de 1903, on voit qu'elle ne contient que 4 espèces et une variété qui n'avaient pas été trouvées dans l'échantillon Boulay, ce sont: Encyonema ventricosum, Fragi'aria Zeilleri, Navicula gigas, Tetracychus Peragalli et sa var. eximia; l'analogie des deux florules est une preuve de l'homogénéité du dépôt. 
DE LA

\title{
VALEUR TAXONOMIQUE DES DLATOMÉES
}

\author{
POUR LA DÉTERMINATION DE L'AGE \\ D'UN DÉPOT FOSSILIFÈRE
}

A propos des données à utiliser pour la détermination de l'âge d'un dépôt fossilifère, nous ferons observer que la florule diatomique est loin d'avoir la valeur taxonomique de la florule phanérogamique, et surtout de la faune fossile; cela tient à ce que les Diatomées ont une aire de dispersion beaucoup plus générale que la faune et les plantes supérieures.

Nous trouvons une première preuve de ce fait, dans la différence numérique très grande qui existe entre le nombre des espèces de Diatomées et celui des plantes phanérogames observées dans un même dépôt; c'est ainsi, par exemple, que pour le beau dépôt remanié de Joursac, la florule diatomique comprend près de 300 espèces, tandis que la florule phanérogamique en compte à peine 75 ; dans le dépôt de Lugarde nous avons observé près de 60 espèces de Diatomées, alors $q$ ue ce dépôt n'a livré à $M$. P. Mlarty qu'une quinzaine de Phanérogames; la même disproportion des deux florules a été constatée à La Garde, à Andelat, etc. ainsi que dans les dépôts remaniés de la Haute-Loire.

Une autre preuve nous est fournie par la comparaison de la florule diatumique des dépôts tertiaires du Massif Central avec celle des dépôts similaires de ré- 
gions éloignées; on peut voir dans notre Mémoire de 1903, page 138, que la flore des argiles miocènes à Diatomées du Cantal contient 80 espèces qui lui sont communes avec celle des dépôts de Sheene (Ecosse), de Rirssby-Calma (Suède) et de Stavanger (Norwège); ainsi, tandis que la flore phanérogamique de ces régions boréales est très différente de celle d'Auvergne, on voit que les flores diatomiques présentent au contraire une grande analogie.

L'aire de dispersion des Diatomées actuelles n'est pas moins étendue qu'elle l'était à l'époque lointaine du Hiocìne, comme le montre l'exemple suivant :

Nous arons reçu, il y a quelques mois, de notre dévoué confrère et correspondant, Frère Arsène, une récolte de Diatomées vivantes, provenant des bords de l'A toyac, près de Puebla (Mexique); or, en examinant une partie de ces matériaux, nous avons constaté que sur une trentaine d'espèces observées, près des deux tiers virent et se multiplient actuellement dans les fossés de la Limagne ou sur les bords de l'Allier, alors que sur 400 phanérogames reçues en même temps que les Diatomées, nous avons trouvé à peine 6 ou 7 espèces appartenant ì la flore européenne, toutes les autres lui sont absolument étrangères.

Cette dispersion générale des Diatomées nous explique la difficulté que les diatomistes éprouvent pour distinguer, par exemple, une florule du Miocène de celle du Pliocène, même en comparant les florules bien datées de deux étages extrêmes; pour notre part, nous devons avouer que la ligne de démarcation nous a toujours paru insaisissable, à cause du grand nombre d'espèces communes aux deux florules comparées.

Les caractères différentiels des florules du tertiaire et du quaternaire sont beaucoup mieux tranchés; celle 
du tertiaire est toujours bien caractérisée par un ensemble de Diatomées qui ne se trouvent plus actuellement à l'état vivant sous le climat du dépôt dans lequel elles ont été observées ; la florule d'un dépôt quaternaire, au contraire, comprend un ensemble de Diatomées que l'on trouve encore à l'état vivant; quant à la distinction des florules respectives des ćtages intermédiaires nous l'avons constamment trouvée indécise et sans valeur taxonomique.

Pour fixer l'âge d'un dépôt le diatomiste a donc besoin d'utiliser les caractères fournis par la faune et la flore phanérogamique, avec les données empruntées ì la stratigraphie, et surtout au complexe lithologique.

La flore phanérogamique nous donne la date précise du remaniement du dépôt ou de sa restratification dans une dépression du sol, ou même dans un lac préexistant; dans ce dernier cas la présence de poissons fossiles mélangés aux empreintes de feuilles, comme à Menat, à Joursac, etc., s'explique naturellement; mais, la date de la flore phanérogamique étant celle de la restratification du dépôt, il est évident qu'elle est postérieure à celle de la formation du dépôt, et, par suite, à celle de sa florule diatomique; les deux florules, ainsi que nous l'avons fait observer ailleurs, peurent même appartenir à deux époques géologiques différentes, comme c'est le cas pour les dépôts du Chambon et de Saint-Saturnin, dont l'une est nettement quaternaire et l'autre tertiaire.

Enfin, l'examen du complexe lithologique nous indiquera très exactement si la formation diatomique est antérieure ou postérieure aux éruptions volcaniques.

Il nous a paru utile de résumer ici ces quelques données relatives à la détermination de l'âge d'un dépôt fossilifère, en raison de l'intérêt qu'elles peuvent ayoir 
pour les paléobotanistes, et pour ceux de nos patients collègues en diatomologie qui s'occupent de préférence des diatomées fossiles.

\section{RÉSULTATS ACQUIS}

Au début de nos recherches (1888), les diatomistes attribuaient à peine une centaine d'espèces à l'Auvergne.

Notre Mémoire de 1893 en mentionnait environ 600, et ce nombre s'est élevé à 950 , par les publications de 1902 et 1903.

Avec le présent Mémoire, nous arrivons au chiffre respectable de 1.100 Diatomées pour la flore d'Auvergne, parmi lesquelles près de 500 espèces ou variétés sont nouvelles pour la flore générale, décrites avec soin et figurées dans 14 bonnes planches.

En ajoutant 62 espèces, trouvées dans les dépôts tertiaires de la Haute-Loire et de l'Ardèche, et qui n'ont pas encore été olsservées dans ceux d'Anvergne, nous obtenons 1.162 Diatomées bien distinctes pour la flore da Massif Central.

Nous nous permettrons d'ajouter que nos labeurs n'ont pas uniquement contribué à la connaissance scientifique des Diatomées de notre province, nous avons aujourd'hui la satisfaction de constater que les vastes dépôts de Celles, d'Auxillac et de Faufouilhoux (Cantal) sont actuellement l'objet d'une active exploitation, et rentrent dans un grand nombre d'applications pour lesquelles les Diatomées fossiles du Cantal sont préférées, avec raison, par les industriels français au Kieselguhr allemand.

DEO scientiarum Domino laus et gloria! 
PLANCHE XIII 


\section{PLANCHE XIII}

Fitr.

1. Amphora protracta Pant. var. gallica nov., page 61 .

2. - araulensis nov, sp., p. 60 .

3. - enoculata nov. sp., p. 55.

4. Cymbella Malinvaudi nov. sp., p. 47.

5. - ovum nov. sp., p. 48 .

6. - Gallaudi nov. sp., p. 49.

7. - gallica nov. sp., p. 56.

8. - - var. gracilis not., p. 22

9. - - var. minor nov, p. 22 .

10. - austriaca Grun. var. recta nov., p. 22 .

11. - tenuis nov. sp., p. 23 .

12. - gastroides Ktz. var. prolongata nol', p. 56.

13. - miocenica nov. sp., p. 62 .

14. - pseudo-turgidulanov. sp., p. 5.

15 - intermedia nov. sp., p. 22 .

16. - pseudo-turgidula var. dilatata nov., p. 23 .

17. - cantalense nov. sp., p. 5.

18. Gomphonema subslavatum Grun. var. major nov., p. 24.

$19 \quad$ - Gallaudi nov. sp., p. 63.

20. Navicula borealis Ktz. var. linear is nov, p. 45.

21 . - subacuta var. minor nov., p. 46.

2. - dubravicensis Pant., var. gallica nov., p. 61.

2.3. - - Berriati F Hérib. var. splendida nov., ๖. 16.

$2+\quad$ ('omplonema Pisciculus nov. $s p$, p. 7.

25. - - fossile nov. sp., p. 8 .

26. - acuminatum Ehrb. var. minutissima nov, p. 7 .

27. - marginatum nov, sp., p. 49.

28 - perfidum nov, sp., p. 7. - La fig. à droite représente une déformation du type.

29-30. Anorthoneis Peragalli nov. sp., p. 26.-29, face supérieure; 30 , face inférieure.

31. Navicula mardansouensis nov. sp., p. 47.

3\%. - halophila var. Eusebioi nov', p. 46 . 
Dialomées d'Auvergne

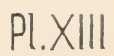

${ }^{1}$

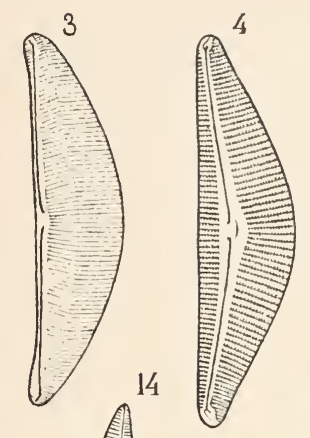

$\beta^{2}$

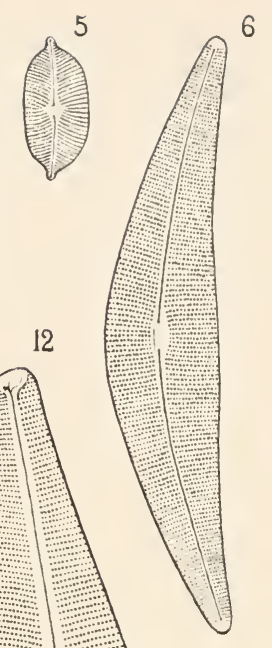

18 管

髾

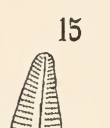

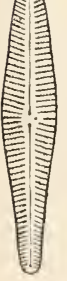

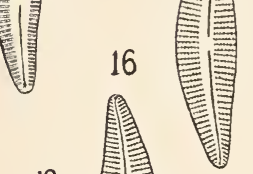

19 䩀

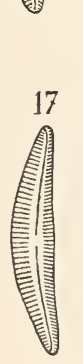

21 铸

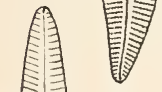
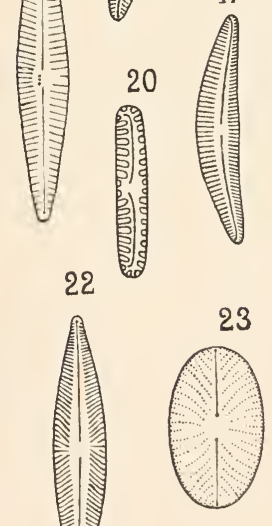

$\frac{600}{1}$
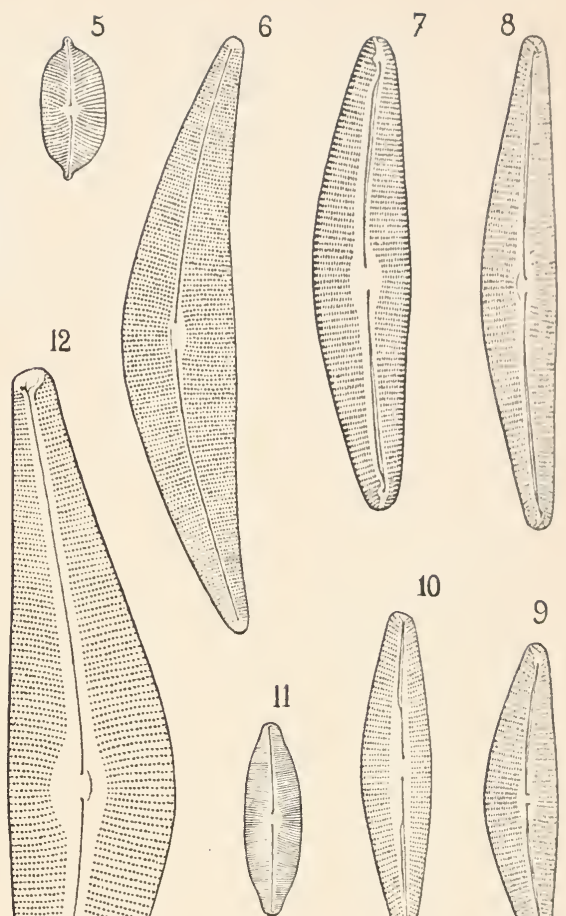

10
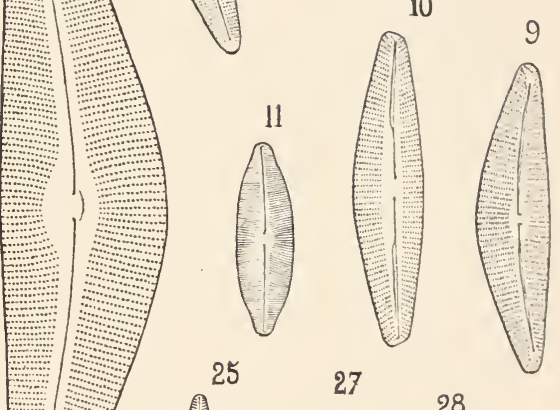

5

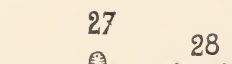

$=$

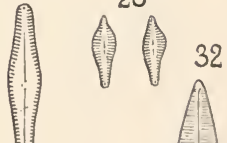

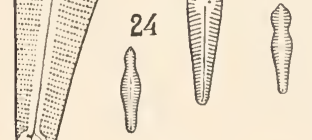
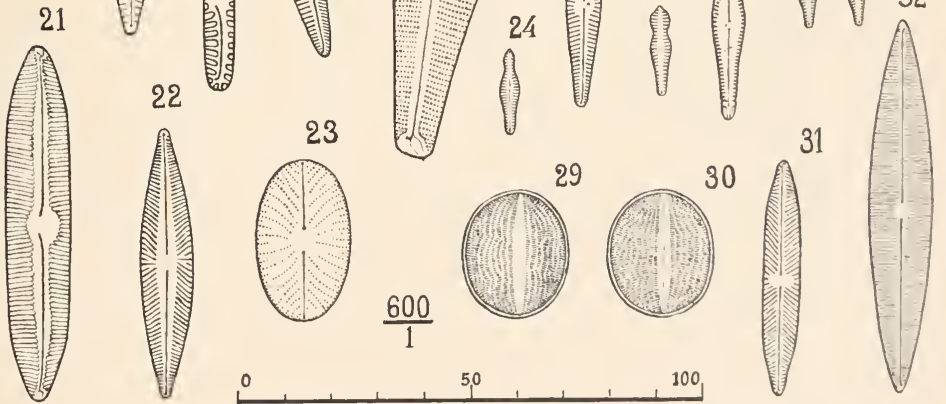

M.Peragallo del. 

PLANCHE XIV 


\section{PLANCHE XIV}

Fig.

1. Eumotia gracilis $\mathrm{W}$. Sm. var robusta nov., page 50 .

2. - arverna nov. sp., p. 33.

3. - pendens nov. sp., p. 6.

4. - divisa nov. sp., p. 35.

5. Fragilaria moissacensis nov. sp., p. 33.

6-7-8. - araulensis nov. sp., p. 64. - 6, face connective ; 7 , forme elliptique; 8 forme étroite et allongée.

9. - lapponica Grun. var. gigantea nov., p. 24.

10-11. - Endocystifera nov.sp., p.64. - 10, face valvaire: 11 , face comnective avec endocyst.

12. Surirella Brunhesi nov'. sp.. p. 29.

13. Staurosira Grunowii Pant. var. minor noc., p. 17.

14. - - var. major nor., p. 18.

15. - - var. triangulata nov, p. 18.

16. - - $\quad$ - var. biangulata nov., p. 18.

17-18. Opephora Glangeandi nov. sp., p. 10. - 17, forme type; 18 , forme plus courte et plus large. - cantalense var. obesa nov., p. 17.

20-21. Tetracyclus glans Pant. var. paucicostata nov., p. 18.

22. - emarginatus var. integriloba nov., p. 52.

23. - major nov. sp., p. 52 .

24. - - var. acuminata nov. sp., p. 52.

25 26. Melosira strangulata nov, sp., p. 31 .

27. - Rieufii now. sp., p. 15.

28-29. - Mauryana nov. sp., p. 8. - 28, face valvaire; 29, face connective.

30. - - var. granulosa nov., p. 58.

31. Gyclotella Charetoni, var. gemmatula nov., p. 25. 
Dialomées d’ Auvergne

PI.XIV
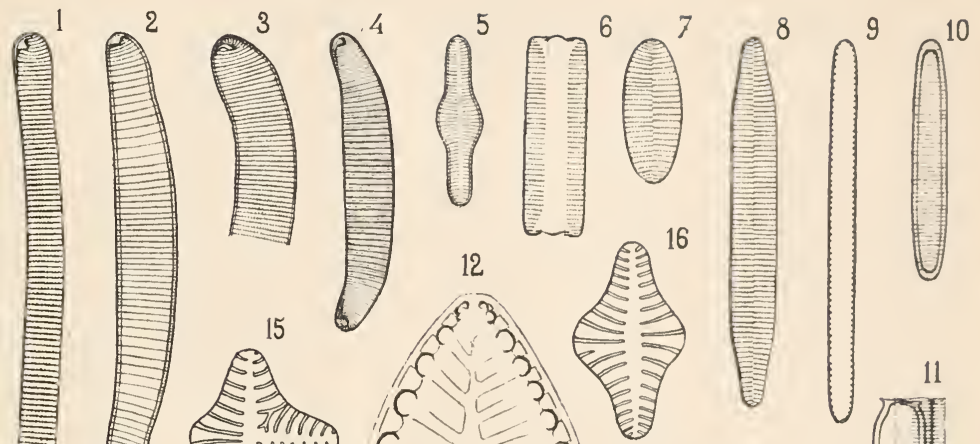

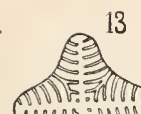
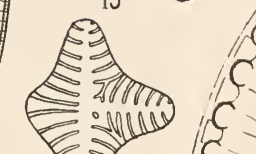

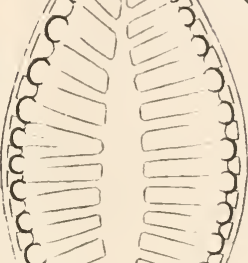
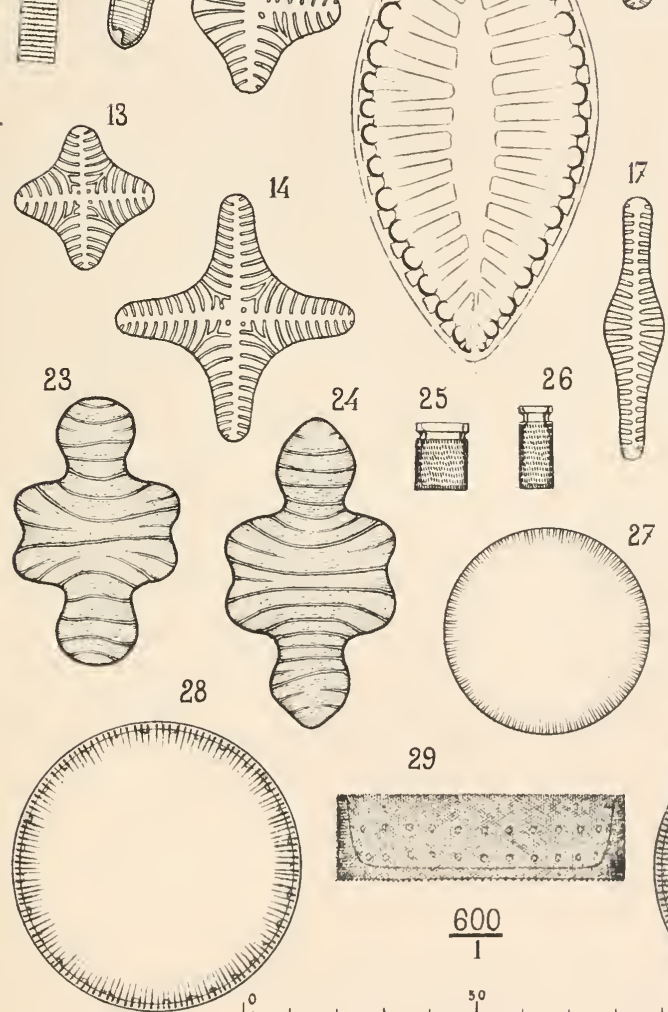

Nov000010

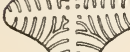

ร2. 14

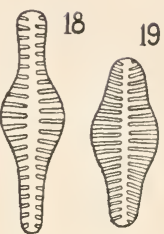
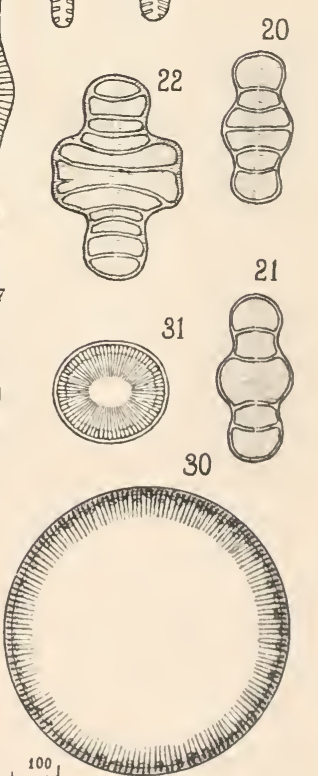

M.Peragallo del. 



\section{TABLE DES MATIÈRES}

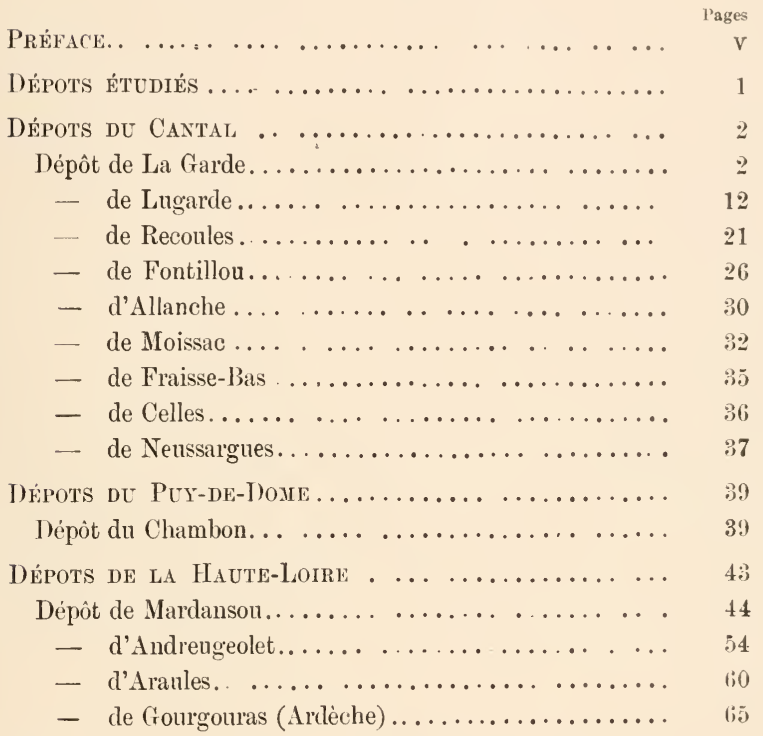

De la valeur taxonomique des Diatomées pour la détermination de l'àge d'un dépôt fossilifère.......... 6 .

Résultats acqnis.................... 70

\section{PLANCHES XIII, XIV}

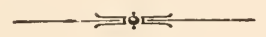



LES

\title{
DIITOMEES FOSSILES
}

\author{
D'AUVERGNE .
}

l'AR

\section{Le Frère HÉRIBAUd JOSEPH}

PROFESSEUR AU PENSIONNAT DE CLERMONT-FERRAND LAURÉAT DE L'INSTITUT' DE FRANCE (Académie des Sciences)

MEMH:E ПOSORAIRE ÉLU DE L'ACADÉMIE DE CLEMMONT-FEIUIMU

ET DE L.I SOCLÉTÉ BOTISIQLE DE FRASCE

MCIEX DIRECTELR DE L'ACADÉMIF IXTERYATWY.ILF:

DE GÉOGR.APHE BOTATQLE

DEO scienliarum Dumino laus et glorit.

\section{IVEC 2 I'LANCHES}

DESSINÉES PAR LE COMMANDANT MAURICE PERAGALLO

\section{Prix : 5 Francs}

CLERMONT-FERRAND

PENSIONNAT

DES FRĖRES DES ÉCOLES CHRÉTIENNES

Rue Godefroy-de-Bouillon
PARIS

LIBRAIRIE DES SCIENCES NATURELLES

PAUL KLINCKSIECK

3, Rue Corneillo, 3 . 



\section{AUTRES PUBLICATIONS BOTANIQLES}

\section{DU F. HERRBAUD JOSEPH}

Le Puy-de-Dôme et le Cantal ou Tableau comparatif de la Flore des deux departements $1 \times 76$.

Florule des terrains arrosés par les eaux minérales de l'Auvergne (1878).

La Flore d'Auvergne (1883), en coilaboration avec mon premier maîlre en botanique, le très regretté $\mathrm{F}$. Gustave.

Les plantes parasites de la Flore d'Auvergne (1889).

Elements de Botanique, comprenant l'organographie, la physiologic et les principales familles végétales; ouvrage en rapport avec les programmes officiels de l'Enseignement secondaire ( 1890 ).

Analyse descriptive des Rubus du Plateau Central (1891).

Supplèment à la Flore d'Auvergne (1892).

Application de l'électricité atmosphérique à l'Agriculture $189 \%$,

Note sur le depôt diatomifère marin du Puy de Mur (1893), en

collaboration avec M. I. Julien, professeur de géologie à l'Lniversité de Clermout.

Les Diatomées d'Auvergne (1893). Ouvrage couronné par l'Inslitut de France (Académie des Sciences).

Nouvelles additions à la Flore d'Auvergne (1895.

Quelques mots sur la Truffe, au point de vue de sa nature, de sa culture et de son importance économique (1896).

Recherches sur les Diatomées des calcaires tertiaires de la Limagne et sur l'origine de ces terrains (1897).

Les Grimmia de la Flore d'Auvergne (1898).

Les Muscinées d'Auvergne (1899). Ourrage courunné par l'Inslitut de France (Académie des Sciences).

La Flore d'Auvergne en 1901 (1902).

sous presse : Un Carex nouveau pour la Flore française (1902), en collaboration avec M. Ernest Malinvaud, Secrétaire gẻnéral de la Société botauique de France.

En mipration : Les Lichens d'Auvergne.

Clermont-Ferrand, typographie et lithographie G. Most-Lot'ts, rue Barbançon. 





